\title{
Abstracts of the 11th Annual Meeting of the Israel Society for Neuroscience
}

Eilat, Israel, December 15 - 17, 2002

Directed convergence of dendrites causes synaptic

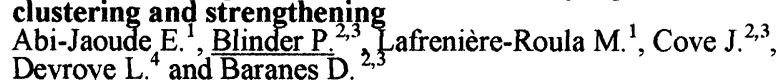
Devroye $\mathrm{L}$. ${ }^{4}$ and Baranes D. ${ }^{2}$

${ }^{I}$ Dept. of Anatomy and Cell Biology, McGill University, Montreal, Canada ${ }^{2}$ Dept. of Life Sciences, Ben Gurion

University of the Negev, Beer Sheva; ${ }^{3}$ Zlowtovski Center for Neuroscience, Be 'er Sheva; ${ }^{4}$ Dept. of Computer Science, McGill University, Montreal, Canada

Synaptic clustering influences synaptic efficacy by increasing cooperative interactions among neighboring synaptic inputs, however, the mechanism of synaptic clustering is unknown. We found that in hippocampal neurons in culture synaptic clustering as well as synaptic strength increase at the point of intersection between dendrites compared to other areas along the dendrites. The clustering in the intersection increases linearly and the strengthening exponentially with an increase in the number of dendrites crossing the intersection. Intersections containing multiple dendrites are frequent ( 4 times more than in computer simulations of randomly distributed dendrites) and are formed by direct growth of dendrites toward pre-existing dendritic intersections. This process is disrupted by inhibitors of synaptic activity and of glutamate receptors. Thus, an activity-dependent mechanism exists that causes dendrites to converge to a single intersection, resulting in an increase in synaptic clustering and synaptic activity at the intersection. This may serve as a mechanism for activity-dependent pre-synaptic plasticity.

In addition to converging, dendrites in the culture tend to grow in parallel, even when separated over large distances, leading to formation of areas where specific dendritic orientations are dominant. This order restricts the location of the dendritic intersections and their synaptic clusters. Thus, directed convergence combined with parallel growth of dendrites shapes the synaptic map in cultured neuronal networks.

Keywords: dendrite, synaptic clustering, synaptic strength

Writing and dysgraphia in ADHD

Adi-Japha E. ${ }^{1}$, Shalev $R^{2}{ }^{2}$, Frenkel L. ${ }^{1}$ and Teicher $M^{1}$

'Bar-Ilan University; ${ }^{2}$ Shaare Zedek Medical Center

Background: Difficulties in writing is a common complaint among children with attention deficit hyperactivity disorder (ADHD).

Objective: To examine whether the problem is one of dysgraphia or attentional problems masquarading as dysgraphia. Methods: Possible sources of writing difficulty tested were: "lingual", associated with reading and expressed mainly in spelling mistakes "peripheral motor-output" designated as the orthographic buffer and expressed in omission or repetition of strokes while writing and "attention deficit" expressed as consistence in production in all domains of graphic production. Participants underwent an extensive reading/writing assessment (Shani \& Ben-Dror Pivotal Test, 2000), and a set of lingual and peripheral agraphia tests using a digitizing tablet. General kinematic performance in graphic production was assessed using a $30 \mathrm{sec}$. repetitious tracing of an ellipse. Participants: Twelve boys with ADHD, aged 11-13 years (unmedicated for at least 1 .week) and controls matched for gender, age I.Q, handedness and socio-economic class. Children were eligible to participate if their IQ was $>85$, and had no known reading difficulty. ADHD children and controls did not differ significantly in reading scores or in the speed of letter naming.

Results: Lingual: ADHD children spelling scores were significantly lower than controls in most spelling tests.

Motor-peripheral: ADHD children had significantly more stroke omission/repetition errors than controls when writing repetitiously similar-letter words.

Attention deficits: ADHD children were significantly less consistent than controls in a repetitious tracing of an ellipse.

One of the digitized tasks was circle drawing, once as a letter once as a number, and once as an eye. Duration of writing/drawing did not differ between groups, but variance of duration over repetitions was larger for ADHD children, especially in the written-letters task

Conclusions: Children with ADHD demonstrated deficits in the lingual and peripheral domains of writing as well as attention deficits in the graphic domain. Although ADHD children were less consistent than controls on graphic tests, for very simple tasks this was significant for writing but not drawing. We conclude that children with ADHD have a true dysgraphia that is exacerbated by the attention deficits. Keywords: dysgraphia, ADHD, writing

Never ending cognitive error correction in the Stroop task in schizophrenia Adi-Japha E. ${ }^{1}{ }_{2}$ Lamschtein $\mathrm{L}^{2}$, Modai $\mathrm{I}^{2}$ and Hirschmann $\mathrm{S}^{2}$ ${ }^{T}$ Bar-Ilan University; Institute for Psychiatric Studies, Sha' ar Menashe Mental Health Center; ${ }^{3}$ College of Judea and Samaria Background: Error detection is known to be impaired in Schizophrenia, but less is known about error-correction.

Objectives: To examine error-correcting activity and correction of errors in the verbal and motor domain in these patients.

Methods: The neurocognitve process of error monitoring and error-correction in schizophrenic patients was examined by using the verbal and handwritten version of the color-naming Stroop task. Eighteen medicated open-ward Schizophrenic patients and controls, matched for gender, handedness, age and education, were asked to 'name the color' and to 'write the color' in two blocks of 24 randomly ordered congruent, incongruent and neutral stimuli. The timing of error correction, if occurred, was recorded in both modalities. In the handwritten version of the task, a digitized tablet recorded spatial and kinematic data.

Results: Error correction activity of schizophrenic patients depends on the modality (verbal/written): The proportion of corrected errors in the verbal domain for patients and controls was similar whereas the handwritten version shows marked differences between the two groups. The corrected handwritten responses in schizophrenic patients persisted significantly longer and appearing at later stages of words. Clinical positive and negative symptoms analysis shows that higher positive symptoms correlate with longer response duration, less accuracy and higher variability in both modalities. Anxiety and poor attention are correlated with less accuracy in handwritten responses (all r's > .4).

Conclusions: Error correcting activity in Schizophrenia differs between modalities.

We hypnotize that the difference between speech and writing feedback processes results in different correcting activity. For handwriting we found an ever-lasting correction. This suggests that in schizophrenia there is a continuous burdened on the thought process by it inability to conclude, especially in the motor domain. We relate these results also to the differentiated function of the anterior cingulated cortex in the verbal and motor domain.

Keywords: schizophrenia, error correction, Stroop, writing

\section{Mechanisms of amygdala modulation of hippocampal}

plasticity

Akirav I. and Richter-Levin G.

Dept. of Psychology, Laboratory of Behavioral Neuroscience,

University of Haifa, Haifa

Basolateral amygdala (BLA) activation by emotional arousal modulates memory-related processes in the hippocampus. We have shown (Akirav and Richter-Levin, $J$ Neurosci 19:10530-5 [1999]) that activating the BLA prior to perforant path (PP) tetanization has a bi-phasic effect on hippocampal plasticity; priming the BLA immediately before PP tetanization results in the enhancement of dentate gyrus (DG)-LTP (an 'emotional tag'), whereas stimulation in a spaced interval results in the suppression of DG-LTP. Here, we aimed to elucidate the mechanisms underlying BLA modulation of DG-LTP and specifically to examine whether the stress hormones norepinephrine (NE) and corticosterone (CORT) are main mediators of the BLA bi-phasic effects. We found that the BLA affects hippocampal plasticity in a complex manner; BLA priming enhanced DG-LTP, and both NE and CORT mediated this effect. Furthermore, we found that ipsilateral BLA spaced activation ( $2 \mathrm{hrs}$ prior to PP tetanization) suppressed DG-LTP and that this suppressive effect was also mediated by NE and CORT. Priming the contralateral BLA enhanced DG-LTP similarly to the ipsilateral enhancement, but neither NE nor CORT mediated this effect. The spaced activation of the contralateral BLA did not suppress DG-LTP. Thus differential mechanisms underlie the ipsilateral and contralateral BLA effects on hippocampal plasticity. 
The BLA modulates hippocampal memory processes presumably via the mediation of the stress hormones $\mathrm{NE}$ and CORT, to establish a diverse memory of the experience. Possibly, at the onset of an emotional event the stress hormones permissively mediate plasticity. However, their prolonged presence in the system may suppress the cognitive response to stress.

Keywords: learning, amygdala, hippocampus, emotions

Memory process and insomnia in the elderly

Akselrod A., Hadad B. and Haimov I.

Dept. of Behavioral Science, Emek Yezreel Academic College

Changes in sleep-wake patterns appear to be one of the hallmarks of biological aging. Elderly persons complain of daytime drowsiness and difficulties in initiating and maintaining sleep. Likewise, aging has a clear effect on learning and memory processes. However, the interaction between sleep disturbances and memory among elderly people remains unclear. This study aims to assess whether insomnia is associated with memory decline in the elderly.

Seventy-three elderly (45 males and 28 females) aged $65-80$ (70.4 5.9 years) who were living independently in the community participated in this study. Sleep was assessed using Mini Sleep Questionnaire, and memory processes were evaluated via the Rey Auditory Verbal Learning Test.

Twenty-three of the 73 subjects $(32 \%)$ had insomnia index greater than 4 in sub-scale of insomnia, indicating insomnia. Performance of the two groups (insomniacs and good sleepers) in the Rey AVLT was compared in order to assess the relationships between insomnia and memory. Generally, the results demonstrated that in almost all categories of episodic memory elderly people who do not have sleep disorders displayed better performance results compared to insomniacs. More specifically, results revealed significant differences between insomniacs and good sleepers in learning, and in resistance to proactive interference, and close to significant in temporal memory.

These findings suggest that insomnia is significantly associated with some aspects of memory decline in the elderly. These aspects include learning, resistance to proactive interference, and temporal memory.

Keywords: aging, memory, cognitive impairment, insomnia

Activation of the human "visual" cortex in the congenitally blind

Amedi A. ${ }_{1}^{1}$, Raz N. ${ }^{1}$, Pianka P. ${ }^{2}$, Hendler T. ${ }^{2}$ Malach R. ${ }^{3}$ and ${ }^{1}$ Hebrew University, Jerusalem $91904 ;{ }^{2}$ Sourasky Medical Center, Tel Aviv 64239; ${ }^{3}$ Weizmann Institute of Science, Rehovot 76100

Previous studies indicated that in congenitally blind subjects the visual cortex is active during Braille reading, suggesting cross-modal recruitment of the occipital cortex for tactile processing. However, Braille reading involves cognitive aspects well as tactile aspects. Using fMRI mapping in congenitally blind people, we find robust activation by both tactile and non-tactile language related tasks, in the calcarine sulcus and a constellation of occipital areas, which correspond to retinotopic visual areas in sighted subjects. No such occipital activation was observed in sighted subjects. The occipital activation pattern was present in the blind when performing an auditory verb generation task or a verbal memory task with substantial overlap between the resultant activation maps. The occipital areas were also activated by Braille reading, but to a lesser extent. Furthermore, analogous to the hemispheric laterality of language areas in sighted subjects, activation of the left occipital cortex was dominant in the blind. These findings suggest that in cases of early onset blindness the occipital cortex undergoes a dramatic change in functionality, such that it now serves high-level cognitive functions.

Supported by Israel Science Foundation grant number 8009

Keywords: plasticity, memory, language, fMRI

\section{Melanopsin In the mammalian circadian system}

\section{Amir S.}

Center for Studies in Behavioral Neurobiology, Dept. of Psychology, Concordia University, 1455 de Maisonneuve Boulevard West, Montréal, QC, H3G IM8 Canada

Photic information for entrainment of circadian rhythms in mammals is transmitted to the circadian clock in the suprachiasmatic nucleus from the retina via the retinohypothalamic tract. The photoreceptors associated with this pathway are unknown, but it is recognized that conventional photoreceptors, rods and cones, do not play a critical role. The opsin-like protein, melanopsin, has been proposed recently as a putative circadian photoreceptor in mammals. This hypothesis is based on the demonstration that melanopsin is expressed in retinal ganglion cells that respond directly to light and that innervate the suprachiasmatic nucleus. In the course of studying a potential role for melanopsin in photic entrainment, we found a fiber plexus immunoreactive to melanopsin in the retinorecipient region of the suprachiasmatic nucleus of the rat. To explore this further, we assessed the expression of melanopsin in the retina and suprachiasmatic nucleus of rats that were treated with the neurotoxin, monosodium glutamate, during the neonatal period. This treatment strongly reduced the number of retinal ganglion cells expressing melanopsin and abolished the expression of melanopsin in the suprachiasmatic nucleus. Because photic entrainment is spared in spite of the massive damage to retinal ganglion cells bought about by neonatal treatment with glutamate, these findings suggest that retinal ganglion cells that project to the suprachiasmatic nucleus and that contain melanopsin in their axon terminals do not play a critical role in photic entrainment in rats. A possible role for these melanopsin containing ganglion cells in mediating the masking effect of light on behavior will be discussed.

Supported by the Canadian Institute of Health Research, the Natural Sciences and Engineering Research Council of Canada, and the Fonds pour la Formation de Chercheurs et l'Aide à la Recherche.

Keywords: melanopsin, retinal ganglion cells, suprachiasmatic nucleus; circadian system

\section{Bifunctional compounds eliciting prolonged}

anti-inflammatory and cholinergic activity

Amitai G., Adani R., Rabinovitz I., Sod-Moriah G., and

Meshulam $\mathrm{H}$

Division of Medicinal Chemistry, IIBR, P.O Box 19, Ness Ziona 74100

Certain CNS disorders such as Alzheimer's disease (AD), and traumatic brain injury (TBI) are accompanied by significant increase in inflammatory markers. Furthermore, $\mathrm{AD}$ and $\mathrm{TBI}$ in humans include reduction in certain cholinergic markers Based on cholinergic hypofunction observed in $A D$ several cholinesterase inhibitors (ChEI) (e.g. Aricept, Exeleon and Reminyl) have been developed and approved for human use We have synthesized a series of bifunctional compounds containing both non-steroidal anti-inflammatory drug (NSAID) and cholinergic up-regulation (CURE) molety. The bifunctional compound IBU-PO inhibits human AChE and $\mathrm{BChE}$ with bimolecular rate constants $1.3 \times 10^{6}$ and $2.0 \times 10^{3}$ $\mathrm{min}^{-1} \mathrm{M}^{-1}$, respectively. The rate constants of ChE's inhibition obtained for its hydrolysis product $\mathrm{PO}-\mathrm{OH}$ are $\mathrm{k}_{\mathrm{i}}=6.4 \times 10^{4}$ and $5.4 \times 10^{4} \mathrm{M}^{-1} \mathrm{~min}^{-1}$, respectively. Thus, NSAID moiety (ibuprofen) contributed significantly to $\mathrm{ChE}$ inhibition rate. In addition, IBU-PO (4 mg/kg, ip) resulted in $50 \%$ inhibition of mouse-blood $\mathrm{ChE}$ prolonging for 5-6 hours. In contrast, pyridostigmine (PYR, 0.13 $\mathrm{mg} / \mathrm{kg}$, im) caused $15-20 \%$ inhibition with a much shorter duration (1-1.5 hour). IBU-PO also caused dose-dependent prolonged hypothermia in mice (3-5 hours). Peripheral anti-inflammatory activity of IBU-PO, DICLO-PO and INDO-PO (5-10 mg/kg, ip) was demonstrated in rat paw edema model. The anti-inflammatory effect prolonged for at least 7 hours post administration. These data demonstrate that the anti-ChE moiety of the bifunctional compounds did not interfere with the NSAID anti-inflammatory activity. Furthermore, IBU-PO reduced the brain edema induced by icv administration of carrageenan in mice and rats. The central anti-inflammatory activity obtained with IBU-PO is probably also responsible for the amelioration of the damage caused by closed head injury in mice. In conclusion, some of the new bifunctional NSAID-CURE compounds demonstrate prolonged anti-inflammatory and cholinergic activity.

Keywords: NSAID, $\mathrm{ChE}$ inhibition

Immunological changes following antisense oligonucleotides treatment in experimental autoimmune Myasthenia Gravis Amitay-Hamra Y. ${ }^{1}$, Evron T. ${ }^{2}$, Berrih-Aknin S. ${ }^{3}$, Soreq H. ${ }^{2}$ and Brenner T

Depts. of Neurology $y^{I}$ and Biological Chemistry ${ }^{2}$, Hadassah

Medical Center and Hebrew Universityof Jerusalem,

Jerusalem: ${ }^{3}$ University Paris-Sud, CNRS3. France

Myasthenia Gravis (MG) is an antibody-mediated, autoimmune neuromuscular disease in which nicotinic acetylcholine receptor (AChR) is the major autoantigen. Typical neuromuscular junction (NMJ) symptoms can be transiently alleviated by acetylcholinesterase (AChE) inhibitors (such as pyridostigmine). However, AChE inhibitors treatment is short-lived and does not prevent disease progression Moreover, $\mathrm{AChE}$ inhibitors were recently shown to elicit 
pronounced and persistent neuronal over expression of $\mathrm{AChE}$, due to feedback up-regulation of the AChE-gene. Our previous studies demonstrate that both MG patients and EAMG rats display significant elevated levels of muscle AChE mRNA and protein. In addition, treatment with 2'-oxymethyl-protected-AS-oligodeoxynucleotides (EN101) suppresses AChE synthesis and avoids feedback response. Consequently muscle activity was rescued and clinical symptoms were improved for a long period. In the present study repeated daily oral treatment with 50 (g/kg EN101 for a month was very effective. This enabled EAMG rats to thrive under conditions where untreated or pyridostigmine-treated animals did not survive. Furthermore, this treatment reduced the anti-rat AChR antibody titer, associated with disease progression and aggravation. In addition, when EN101 was added in vitro to T-cells reactive towards autoantigen-T-AChR or mitogens, T-cell proliferation was markedly suppressed. Our results show the beneficial effect of EN101 treatment on EAMG clinical and immunological parameters and highlight potential advantage of gene-targeted drug therapy.

Keywords: acetylcholine receptor, acetylcholinesterase, experimental autoimmune myasthenia gravis, antisense-treatment

\section{Single dose intravenous valproate or fosphenytoin in acute} mania

Applebaum J. Phrolov K., Levine J. and Belmaker RH Ministry of Health, Beer Sheva Mental Health Center, Faculty of Health Sciences, Ben Gurion University of the Negev, Beer Sheva

Objectives: High-dose loading with oral and intravenous valproate has been reported to be therapeutic in mania over 48-72 hours. We hypothesized that very high dose iv valproate might have even more rapid effects equivalent to effects in status epilepticus that occur within 20 minutes. Since several anticonvulsants are therapeutic in mania and since acute mania requires rapid and intensive treatment, we hypothesized that intravenous high dose phenytoin might be acutely antimanic. A new prodrug of phenytoin, fosphenytoin, with few cardiac or local vein side effects was used.

Methods: Seven patients with mania and minimal prior drug treatment were given iv valproate $20 \mathrm{mg} / \mathrm{kg}$ over 30 minutes. Seven patients with mania and minimal prior drug treatment were given iv fosphenytoin $5 \mathrm{mg} / \mathrm{kg}$ over 10-20 minutes.

Results: No antimanic effects were observed over 120 minutes of observation. There were no side effects

Conclusion: Slowly evolving biochemical changes, perhaps at the gene level, may be required for the antimanic effect of anticonvulsants.

Keywords: intravenous valproate, anticonvulsants, mania treatment

The lipophilic transition metal chelator DP-109 attenuates asymmetric rotations in the 6-hydroxydopamine partial lesion model of Parkinson's disease in adult rats

Aran A., Friedman J.E., Shapiro I., Angel I. and Kozak A.

D-Pharm, Ltd., Kiryat Weizmann Science Park, Bldg. 7, Rehovot 76123

Perturbations in the homeostasis of transition metals such as copper, iron and zinc can cause oxidative stress in neurons. These metals are considered to be involved in neurodegenerative disorders such as Parkinson's and Alzheimer's disease. Antioxidants and metal chelators have been found to be beneficial in various models of these diseases, but are problematic due to a poor safety profile and low rate of penetration across the blood brain barrier. To address this problem, we have developed a family of lipophilic chelators selectively activated in the vicinity of cell membranes. The lead compound, DP-109, was designed to chelate bivalent ions such as $\mathrm{Zn}, \mathrm{Cu}, \mathrm{Fe}$ and $\mathrm{Ca}$. DP-109 was tested in the unilateral 6-OHDA substantia nigra lesion model using adult male Wistar rats. Five days post-lesion animals were screened for apomorphine-induced rotations over a $5 \mathrm{~min}$ period. Responding animals subsequently received either DP-109 $(10-500 \mathrm{~g} / \mathrm{kg})$ or vehicle daily, p.o., for a period of up to 45 days. Animals were tested weekly for apomorphine-induced rotations. Results were standardized and the increase in asymmetric rotations, indicating progressive neurodegeneration, was calculated. Vehicle treated animals increased their rotations approximately 15 -fold within 30 days. DP-109 treated animals demonstrated significantly fewer rotations in a dose-dependant manner. DP-109 $(100 \mathrm{~g} / \mathrm{kg})$ significantly attenuated $(p<0.05)$ both the rate of increase and number of rotations by $70 \%$. We suggest that DP-109 represents a new class of compounds that might be effective in treating neurodegenerative disorders.
Supported by: D-Pharm, Ltd.

Keywords: lipophilic transition metal chelator, Parkinson's disease, 6-OHDA

\section{Active immunization towards Prion disease}

Arbel M. and Solomon B.

Dept. of molecular microbiology and biotechnology, faculty of life science, Tel-Aviv university, Tel Aviv 69978

The transmissible spongiform encephalopathies (TSE) or better known as prions, are rare neurodegenerative diseases which include scrapie in sheep, BSE in cattle and Kuru, Creutzfeldt-Jakob disease, Gerstmann-Strussler- Schienker syndrome (GSS) and fatal familial insomnia (FFI) in human. It is believed that prion propagation is caused by refolding of a normal endogenous glyco- protein called prion protein $(\mathrm{Pr} \mathrm{Pc}$ ) PrPc is expressed mostly in the central nervous system and lymphoid tissue. In the course of the disease PrPc changes its structure into an abnormal (-sheet rich structure termed PrPsc which is the only known particle in the prion infectious agent. The abnormal protein is neurotoxic and leads to death within few month. As for today there is no effective therapeutic agent to prion diseases

Antibodies are known to act as chaperones and are able to stabilize protein structure and/or induce conformational changes. Thus, our working hypothesis is that site directed antibodies towards PrP may interfere with aggregation processes and/or inhibit prion replication.

We developed in our lab an active immunization procedure towards human Helix 1 of the whole prion protein. Helix 1 was chosen since it appears to be a putative key position in the protein induced conformational changes.

In order to overcome the poor immunogenicity of short peptides in general and the high sequence similarity between the human and mouse peptides in particular, we chose the MAP (Multiple Antigen Peptides) as the antigen of choice. The MAP is a branching structure of lysine residues coupled to the desired peptide.

The immune response that our peptide elicits was unexpectedly high. Antibodies raised were of IgG isotype. The antibodies that were produced recognize the whole protein and are now being tested for their ability to inhibit the PrPc to PrPsc conversion in cell culture model.

Keywords: PrP (prion protein), chaperons, MAP

Sub-millisecond precision of the input-output transformation function mediated by fast sodium dendritic spikes in basal dendrites of CA1 pyramidal neurons Ariav G Polsky A and Schiller J.

Dept. of Physiology, Technion Medical School, Haifa

The ability of cortical neurons to perform sub-millisecond scale computations has been shown to be important for encoding of information in the cortex (1-2). This ability necessitates involvement of special mechanisms in order to overcome the relative long membrane time-constant and the significant dendritic filtering of excitatory postsynaptic potentials (EPSP) in cortical neurons. Here we show that coincident activation of closely spaced basal inputs in CAl pyramidal neurons resulted in significant sharpening $(872 \pm 375 \%)$ and supra-linear amplification $(156 \pm 37 \%)$ of the summed potential, as compared to the individual EPSPs. These phenomena are mediated by initiation of a local dendritic spike composed of an early fast sodium spike followed by a slower NMDA spike When paired with apical EPSPs fast local basal sodium spikes significantly improved the temporal precision of output action potentials by markedly decreasing the temporal jitter of action potentials (from $2.17 \pm 1.12$ to $0.28 \pm 0.17 \mathrm{msec}$ )

Our findings indicate that local dendritic basal spikes enable coincidence detection of closely spaced synaptic inputs, and significantly improve the temporal output precision of $\mathrm{CAl}$ neurons. As such they may serve as a cellular basis for sub-millisecond temporal coding in cortical neurons.

Keywords: dendrites, temporal coding, synaptic integration, dendritic spikes

$\mathrm{Na}^{+}$channel properties determine the functionally optimal oscillation frequency of neocortical pyramidal neurons Astman N. ${ }^{1}$, Gutnick M.J. ${ }^{2}$ and Fleidervish I.A.

${ }^{1}$ Zlotowski Center for Neuroscience. Ben Gurion University of the Negev, Beer-Sheva; 'Koret School of Veterinary Medicine. The Hebrew University of Jerusalem. Rehovot 76100

One prominent feature of neocortical pyramidal neurons is their tendency to oscillate at specific frequencies. Recently, attention has focused on the functional significance of such oscillations for modulating the excitability of the somato-dendritic membrane. During oscillations, the moment-to-moment availability of $\mathrm{Na}^{+}$current, which is the major determinant of neuronal excitability, depends on the 
dynamic properties of the $\mathrm{Na}^{+}$channel. We used the cell-attached configuration of the patch-in-slice technique to measure the relevant $\mathrm{Na}^{+}$channel parameters in somata of neocortical Layer 5 cells. Unlike in most previous studies, these experiments were performed at physiological temperature, since the dynamic properties of interest are temperature dependent. Warming the preparation from room temperature to $36^{\circ} \mathrm{C}$ did not affect the activation curve, but caused the steady-state inactivation curve to become steeper. At sub-threshold potentials, time constant of recovery from inactivation was much slower $(4-6 \mathrm{~ms})$ than that predicted by the Hodgkin-Huxley model $(<0.3 \mathrm{~ms})$. The number of "ready-to-open" channels during oscillation was measured by applying sine wave voltage commands of constant amplitude $\left( \pm 5 \mathrm{mV}\right.$ from $\left.\mathrm{V}_{\text {rest }}\right)$ and different frequencies $(5-100 \mathrm{~Hz})$, superimposed by a brief depolarizing pulse. $\mathrm{Na}^{+}$channel availability was lowest at $5 \mathrm{~Hz}$ and increased sharply as a function of frequency, reaching a maximum at around $40 \mathrm{~Hz}$. Dendritic current-clamp recordings revealed a parallel frequency-dependent increase in the amplitudes of back-propagating action potentials. We conclude that the hi-pass filtering properties of the $\mathrm{Na}^{+}$channel determine optimal oscillation frequency.

Keywords: oscillation, $\mathrm{Na}$ channel, neocortical neuron

Neonatal immunization of mice with self-proteins residing in the site of glutamate toxicity eliminates their ability as adults to withstand the toxicity

Avidan $\mathrm{H}^{1}{ }^{1}$, Kipnis $\mathrm{J} .{ }^{1}$, Caspi R. ${ }^{2}$ and Schwartz $\mathrm{M}^{1}{ }^{1}$

${ }^{T}$ Dept. of Neurobiology, Weizmann Institute of Science,

Rehovot 76100; ${ }^{2}$ National Institute of Health Bethesda, USA

Over the last few years, studies from our laboratory have demonstrated that after an insult to the central nervous system (CNS), the body harnesses an immune response against specific self-proteins residing in the damaged CNS in order to attenuate the damage caused by the injury-induced emerging destructive self-compounds at the lesioned site. In the present study we show that the ability of CNS neurons to withstand glutamate toxicity, one of the major factors in neurodegenerative conditions, is diminished if the individual is deprived of the ability to manifest an immune response against proteins residing in the stressed tissue. This finding argues in favor of autoimmunity as a protective physiological response to a threat originating from within the body (as opposed to an exogenous threat), and emphasizes that the immune system is the backup supportive mechanism for the central nervous system when the latter exceeds the maintaining capacity of the resident neural cells.

Keywords: autoimmunity, neuroimmunology, vaccination, EAU

Early exposure to stress modulates the response to stress in the adult rat

Avital A. and Richter-Levin G

Dept. of Psychology and The Brain \& Behavior Research Center, University of Haifa, Mount Carmel. Haifa 31905

It has been proposed that exposure to stress during early childhood may disturb emotional and cognitive functioning in adulthood.

We examined the behavioral consequences of early exposure to stress, and in particular, on the ability to cope with stress in adulthood

Post-weaning and Pre-puberty, at the age of 4 weeks, rats were placed on a platform in the middle of a water pool, for 30 minutes (Platform stress)

In the $1^{\text {st }}$ experiment, we used as late stress the Host-intruder interaction at the age of 8 weeks. All rats were then tested in an open-field the Morris water-maze and the startle-reflex test.

In the $2^{\text {nd }}$ experiment adult rats were trained under stress condition (two-way avoidance task) in a shuttle box, followed by affective examination

We report here that an early exposure to stress led to elevated levels of anxiety, as measured in the open-field and startle response tests. However, the rats that were exposed to the combination of early and late stress exhibited the highest level of anxiety. In addition, and as was previously reported the early + late stress group acquired a stressful spatial learning task faster. Furthermore, rats that were exposed to stress early in life did not learn the two-way avoidance task in adulthood.

These findings suggest that an early exposure to stress may have lasting effects on both emotional and cognitive abilitis in adulthood, as is indicated by its effects on anxiety levels and on the performance in a spatial learning and two-way-avoidance tasks.

Supported by a grant 52/2000 from the Israel Foundation Trusties to G. R-L

Keywords: early stress, anxicty, learning
Post-weaning pre-pubery exposure to stress effects on DHEA and DHEA-S in the rat hypothalamus and entorhinal cortex Avital A. ${ }^{1}$, Jacobson $S^{1}{ }^{1}$, Maayan $\mathrm{R}^{2}{ }^{2}$, Ram E. ${ }^{2}$, Weizman $A{ }^{2}$ and Richter-Levin $\mathrm{G}$.

${ }^{I}$ Dept. of Psychology and The Brain \& Behavior Research Center, University of Haifa, Mount Carmel, Haifa 31905; ${ }_{2}^{2}$ Felsenstein Medical Research Center, Rabin Medical Center, Beilinson Campus, Petah Tiqva

Recent evidance support the hypothesis that exposure to stress or trauma during early childhood may disturbe the formation of functional brain pathways, in particular of the limbic circuits.

Thus, we examined the effects of an early exposure to stress on behavior and neurosteroids levels in adulthood. Post-weaning and Pre-puberty, at the age of 4 weeks rats were placed on a platform in the middle of a water pool, for 30 minutes. As late stress, we used the Host-intruder interaction at the age of 8 weeks. All rats were then tested in an open-field, the Morris water-maze and the startle-reflex test. Immediately after the last behavioral test, rats were decapitated and the hypothalamus and entorhinal cortex (EC) were removed for measuring the neurosteroids DHEA and DHEA-S

We report here that an early exposure to stress led to elevated levels of anxiety, as measured in an open-field and startle response tests. In a spatial learning task, the early stress group didn't reach criterion. In the hypothalamus, early exposure to stress did not alter either DHEA or DHEA-S levels, whereas exposure to stress in adulthood increased their levels. On the other hand, in the EC, early exposure to stress increased both DHEA and DHEA-S levels, whereas exposure to stress in adulthood decreased their levels compared to the control and early stress groups.

These findings indicate that an early exposure to stress has a differential effect on DHEA and DHEA-S levels in the hypothalamus versus EC. The relevance of which to the elevated anxiety is yet to established.

Supported by a grant 52/2000 from the Israel Foundation Trusties to G. $R-L$

Keywords: early stress, anxiety, neurosteroids

Effect of the dextrocannabinoid PRS-211,092 on gene expression in brain ischemia induced by MCAO Avraham A., Efroni G., Oni-Biton E., Bar-Joseph A. Berckovitch Y., Azulay M., Garzon A., Elgamiel R. Greenberg O., Amselem S. and Fink G.

Pharmos Ltd, Kiryat Weizmann, Rehovot 76326

The non-psychoactive dextrocannabinoid, PRS-211,092, was recently demonstrated to be an effective neuroprotectant agent in brain ischemia induced by middle artery cerebral occlusion (MCAo) in rats. The mechanism of action, which contributes to its protective effect in brain ischemia is unknown. Here we have investigated the effect of PRS-211,092 on gene regulation in mouse brain after MCAo. Mice were subjected to transien MCAo for $90 \mathrm{~min}$ and then immediately injected with PEG-Ethanol (vehicle only group) or $5 \mathrm{mg} / \mathrm{kg}$ PRS-211,092 i.v. Brains were removed 18 hours after MCAo and the expression of several genes determined in the ipsilateral cerebral hemisphere using real time RT-PCR. Gene expression was normalized to the cyclophilin gene and the level of expression calculated relative to that in sham operated control animals. In the animals treated with PRS-211,092 there was a reduction in expression of the genes for COX-2 (PGE-2 synthetase) by $48 \%$, MCP-1 (monocyte chemoattractant protein-1) by $63 \%$ and IL-2 (Interleukin-2) to a level below that in sham-operated animals. In contrast, IL-10 gene expression was increased by $229 \%$ in the PRS-211,092 treated animals in comparison with that in animals treated with vehicle alone. The reduction in COX-2, MCP-1 and IL-2 gene expression, together with the induction of IL-10 gene expression by PRS-211,092 are likely to play a key role in the neuroprotective effect of this dextrocannabinoid in MCAo-induced brain ischemia.

Keywords: MCAo, neuroprotection, gene expression, dextrocannabinoid

Encoding of radial object position by rat whiskers: recordings from the trigeminal ganglion

Bagdasarian K. Szwed M. and Ahissar E.

Dept. of Neurobiology, Weizmann Institute of Science, Rehovot 76100

We investigated how neurons in the trigeminal ganglion of anesthetized rats respond to whisking against object inserted at different radial positions i. e. along the whisker length Rhythmic $5 \mathrm{~Hz}$ whisker movements similar to spontaneous whisking were induced by electrical stimulation of the facial motor nerve. A vertical pin-shaped object was placed inside the 
whisking field at different radial positions. We recorded single-unit responses and captured video-images of the whisker at $1 \mathrm{~ms}$ resolution. This allowed us to accurately correlate the moment when the whisker touched the object with neural events.

We report here preliminary results from 'object detectors', i. e. single units that responded selectively to the contact between whisker and object (see accompanying abstract by Szwed et al.). We examined the responses of these neurons to objects positioned at three radial distances: $30 \%, 60 \%$, and $90 \%$ of whisker length. We analyzed four response variables: spike-count (number of response spikes per whisking cycle), amplitude (peak firing-rate), duration (of response burst), and latency to $1 / 2$ of peak response. These data show that radial object position is encoded most consistently by spike-counts, less by amplitude, and much less by duration or latency. As radial distance increased, spike-count and amplitude decreased. In contrast, as reported in the accompanying abstract, horizontal position was most consistently encoded by latency. These preliminary results suggest that horizontal and radial coordinates of object position are encoded differently: primarily by spike-count in the radial direction and primarily by latency in the horizontal direction.

Supported by ISF grant 377/02-1.

Keywords: object localization, neural code, sensory encoding

Multi-unit recordings from the gustatory cortex of the freely behaving rat reveal differential response to familiar and unfamiliar taste during a distinct phase of the response Bahar A., Dudai Y. and Ahissar E.

Dept. of Neurobiology, Weizmann Institute of Science, Rehovot 76100

The ability to distinguish an unfamiliar tastant from a familiar one is crucial to the animal's survival, as the unfamiliar may poison. Ample molecular and cellular data support the hypothesis that the gustatory cortex (GC) plays an important role in the neural processing underlying detection of unfamiliar tastants, and transforming them to familiar stimuli. We investigate the neural correlates of gustatory unfamiliarity/familiarity encoding in the $\mathrm{GC}$ of the freely behaving rat. To this end, we employ chronically implanted multi-wire electrodes to record the extracellular activity of multiple units in the GC of rats, while they lick unfamiliar or familiar solutions. We calculate the ratio of responses to taste vs. water and compare them between exposures to unfamiliar and familiar tastants. We find that most recorded units demonstrate a typical lingering response $(7 \mathrm{sec})$ to $1 \mathrm{sec}$ of licking. The ratio of response to taste vs. water, in the initial 2 sec following drinking onset, is independent of whether the taste is unfamiliar or familiar. This ratio, however, increases significantly during the following $5 \mathrm{sec}$. Thus, our data imply that the GC responds differentially to familiar vs. unfamiliar tastants during a distinct phase of the neural response. Our data corroborate the notion that multiple taste attributes, including familiarity, are encoded in the GC, and that at such attributes undergo experience dependent modifications.

Supported by the Minerva stiftung and the Human Frontiers Science Program Organization (to Y.D.), and by the Abramson Family foundation and the Edith C. Blum Foundation (to E.A.) Keywords: taste, electrophysiology, novelty, neural code

Non-conventional vaccination countracts the ongoing damage of chronic neurodegenerative diseases

Bakalash S. and Schwartz M.

Dept. of Neurobiology, Weizmann Institute of Science, Rehovot 76100

Recently it was shown in our laboratory that immune intervention by passive or active vaccination directed to tissue specific abundant antigens protect neurons from consequences of insult within the same tissue. Thus myelin associated antigens protect from consequences of acute axonal injury being a crush of the optic nerve or spinal cord contusion (Moalem et al Nat Med, 1999 Hauben et al J Neurosci 20,6421-6430 [2000]. In contrast, retinal-derived abundant peptides protect from glutamate toxicity inflicted directly to the retinal ganglion cells (Mizrahi et al., J. Immunol. 2002). Vaccination with the random copolymer, Copaxone (Cop-1), circumvented the tissue specificity barrier of protective vaccination. In this work we use an animal model for chronic neurodegenerative disease characterized by high intra ocular pressure (IOP) and progressive optic nerve degeneration resulting in gradual visual ficld loss. We show that autoimmune neuroprotection is effective in chronic conditions. We show that rats with high IOP are amenable to protection by vaccination with antigens residing in the retina and not in the optic nerve. We show that the efficacy of Cop- 1 is dose and regimen dependent and that its effect is superior to pharmacological intervention such as that achieved with $\alpha 2$-adrenoreceptor agonist. It is suggestive that vaccination help homing of $T$ cells to the site of damage. Such T cells are locally activated by the relevant antigen presenting cells and in turn amplify and regulate the tissue ability to fight against the local threat.

Keywords: glaucoma, immune neuroprotection, Cop-1

A monoclonal antibody to the binding site of acetylcholine receptor cross-reacts with the binding site of snail acetylcholine binding-protein

Balass M. ${ }^{1}$, Katchalski-Katzir E. ${ }^{1}$, Smit AB. ${ }^{2}$ and Fuchs $\mathrm{S}^{3}$ Depts. of ${ }^{1}$ Biological Chemistry and ${ }^{3}$ Immunology, Weizmann Institute of Science, Rehovot 76100; ${ }^{2}$ Dept. of Molecular and Cellular Neurobiology, Research Institute Neurosciences, Vrije Universiteit, Amsterdam, the Netherlands

Monoclonal antibody (mAb) 5.5 raised against Torpedo nicotinic acetylcholine receptor (AChR) is specific for the binding site of $\mathrm{AChR}$ and competes with the binding of $\alpha$-bungarotoxin ( $\alpha$-BTX) and other cholinergic ligands to the receptor. Here we show that $\mathrm{mAb5} .5$ binds to snail acetylcholine binding-protein (AChBP), the structure of which has recently been deciphered. As the binding affinity of mAb5.5 to AChBP is considerably higher than that of $\alpha$-BTX to AChBP, we focused on the capability of this antibody to bind either AChR and or AChBP. We thus employed peptides corresponding to the binding site loops of Torpedo and human $\mathrm{AChR}$, and of snail AChBP. We tested the potency of the peptides to compete with the binding of mAb5.5 to either Torpedo AChR or snail AChBP. A peptide corresponding to amino acid residues 187-200 of Torpedo AChR $\alpha$-subunit (WVYYTCCPDTPYLD, T $\alpha 187-200$ ) inhibits the binding of mAb5.5 to both Torpedo AChR and to AChBP $\left(\mathrm{IC}_{50}\right.$ values of $10^{-5}$ and $10^{-6} \mathrm{M}$, respectively). A peptide corresponding to the homologous residues in AChBP (SVTYSCCPEAYED BP182-194) inhibits only the binding of mAb5.5 to its parental molecule, AChBP $\left(\mathrm{IC}_{50}\right.$ of $\left.10^{-4} \mathrm{M}\right)$. Likewise, the peptide corresponding to amino acid residues 187-200 of human AChR $\alpha$-subunit (SVTYSCCPDTPYLD, H $\alpha 187-200$ ), that has a high sequence similarity to the AChBP peptide (BP182-194), also inhibits only the binding of $\mathrm{mAb} 5.5$ to $\mathrm{AChBP}\left(\mathrm{IC}_{50}\right.$ of $\left.10^{-5} \mathrm{M}\right)$ Our findings demonstrate that mAb5.5 interacts directly with the ligand-binding site of $\mathrm{AChBP}$, and makes this antibody a good candidate for analyzing the ligand-binding site of snail AChBP as well as of proteins that bind acetylcholine, in general

Keywords: nicotinic acetylcholine receptor; acetylcholine binding site; acetylcholine binding protein

\section{Pitch discrimination as a marker of reading and reading} related cognitive difficulties

Banai $K^{1}$ and Ahissar $M^{1,2}$

Interdisciplinary Center for Neural Computation, Hebrew University of Jerusalem, Jerusalem; ${ }^{2}$ Dept. of Psychology, Hebrew University of Jerusalem, Jerusalem

Previous studies documented strong correlations between auditory frequency discrimination thresholds (JND) and "high-level" cognitive abilities among reading disabled and normally reading adults. In this study we screened a regular class and a 'learning-disabled' (LD) class from two junior high-schools, respectively. In addition to reading, phonological awareness and memory we measured frequency discrimination. Two methods were used: pitch judgment (hi-lo) and similarity judgment (same/different-SD). In both, tones were $50 \mathrm{~ms}$ long with is inter-stimulus-interval. In each trial one tone was of $1000 \mathrm{~Hz}$. In the hi-lo method the other tone was always higher. In the SD method the other tone was higher in half the trials and same in rest. We asked whether frequency JNDs are a good marker of reading related abilities. Replicating the adult findings, using the hi-lo paradigm, we found that the disabled readers in both classes $10 \%$ and $50 \%$ in the regular and LD class, respectively) were also those with the highest frequency JNDs $(>30 \%)$ and with particularly impaired verbal memory Hi-lo Frequency JNDs were correlated with verbal memory scores, but not with non-verbal memory. Surprisingly, JNDs on the SD task did not correlate with any of the reading or reading related measures, nor with the hi-lo JNDs. The finding that behavioral paradigm is an important factor in the correlation to memory and reading scores suggests that these relations do not simply result from variability in low-level auditory processing. Although the causal direction is not yet clear, we suggest that hi-lo frequency discrimination may serve as a useful screening tool for memory related learning difficultics in schools.

Keywords: auditory processing, reading disability, frequency discrimination 
Plasma membrane targetting of syntaxin 1a is enhanced following expression of $\mathrm{M}_{2}$ muscarinic acetylcholine receptors in PC12 cells

Baram D., Shilkrot R. and Linial M.

Dept. of Biological Chemistry, Alexander Silberman Institute of Life sciences, The Hebrew University, Jerusalem.

In mammalian brain, the $\mathrm{m} 2$ subtype of the presynaptic muscarinic acetylcholine receptors $(\mathrm{mAChR})$ are predicted as autoreceptors for ACh transmission. Preliminary results in our laboratory suggest a functional and molecular link between syntaxin, a crucial member of the exocytotic apparatus, and the $\mathrm{m} 2 \mathrm{mAChR}$. This link is mediated by the alpha subunit of the Go protein.

A key component in the functionality of $\mathrm{G}$ protein-coupled receptors is in their life cycle: intracellular trafficking, targeting to the membrane and internalization. Herein, we show that in the neuroendocrine secretory cell line PC12, expression of $\mathrm{m} 2 \mathrm{mAChRs}$ results in efficient targeting of the receptors to the cell surface while altering the subcellular profile of syntaxin. In control PC12 cells, that do not express endogenous $\mathrm{m} 2 \mathrm{mAChR}$, syntaxin expression is partitioned between the Golgi and the plasma membrane. However, in cells expressing exogenous $\mathrm{m} 2$ receptors, syntaxin is primarily localized to the plasma membrane. This phenomenon could be observed by both immunofluorescence staining and following subcellular fractionation of the cells. Furthermore, m2 mAChRs expression in these neuroendocrine cells causes a dramatic decrease of synaptophysin from the secretory granule fraction, whereas the protein remains located exclusively to a lighter membrane fraction that represents the small synaptic-like vesicles. The data suggest that $\mathrm{m} 2 \mathrm{mAChR}$ receptors are directly involved in the efficient trafficking of synaptic proteins, including syntaxin. The involvement of the G-proteins in the process and the physiological consequence of the observed phenomena are currently being investigated. Keywords: muscarinic $\mathrm{ACh}$ receptor, syntaxin, targeting, secretion

\section{Dynamic changes in mitochondrial function after closed} head injury in mice

Barash V. ${ }_{2}$ Barnea D. ${ }^{1}$, Alexandrovich A. ${ }^{2}$, Beni $\mathrm{SM}^{2}$ and Shohami E.2.

${ }^{1}$ Dept. of Clinical Biochemistry, Hadassah Hospital; ${ }^{2}$ Dept. of Pharmacology, Hebrew University, Jerusalem

Traumatic brain injury is associated with a rapid burst of reactive oxygen species (ROS), leading to oxidative stress. Additionally, ischemia and energy failure occur within minutes to hours after trauma. Mitochondria dysfunction is a major common pathway to these pathological events. The present study examines the fuctional activity of the mitochondria by measurment of ATP producion at various time intervals after closed head injury (CHI) in mice. CHI was induced by a weight-drop device on 8-10 week old male Sabra mice, as described earlier. Mitochondrial fractions were isolated at 1,4 or $24 \mathrm{~h}$ after $\mathrm{CHI}$, and ATP production was followed by addition of glutamate $(10 \mathrm{mM})$ and malate $(0.5 \mathrm{mM})$ for 5 mins. The accumulating ATP was measured spectrophotometrically. In addition, enzymatic activities of complex I-III and of complex IV were determined in the same preparations. A significant decrease in ATP production was noticed already at $1 \mathrm{~h}$ post $\mathrm{CHI}(\sim 22 \%$ decrease form basal activity, $\mathrm{p}<0.05)$. A transient return towards baseline activity was found at $4 \mathrm{~h}$ ( $\sim 8 \%$ decrease, not significant), which was later declined again, and at $24 \mathrm{~h}$ a decrease of $\sim 20 \%$ was recorded $(p<0.05$ ). Interestingly, a similar bi-phasic pattern was reported for the endogenous low-molecular weight antioxidants, in the same model. The enzyme activity assays revealed no changes in NADH-cytochrome-c oxidoreductase and in cytochrome-c oxidase at any time tested. Citrate synthase activity, a marker for mitochondrial content, was also unaffected by $\mathrm{CHI}$. We propose that $\mathrm{CHI}$-induced effects which interfere with ATP-producing pathways by the mitochondria, contributes to the aggravation of cellular damage.

Keywords: traumatic brain injury, mitochondria, oxidative phosphorylation
High expression of apolipoprotein -E and Cathepsin B/D in spinal cords of amyotrophic lateral sclerosis patients and mice expressing human SOD mutation

Barhum Y. ${ }^{1}$, Ransmayr G. ${ }^{2}$, Melamed E. ${ }^{1}$ and Offen D. ${ }^{1}$

${ }^{I}$ Dept. of Neurology, Felsenstein Medical Research Center, Rabin Medical Center, Petah-Tiqva 49100 and Sackler School of Medicine, Tel Aviv University, Tel Aviv 69978; '2 Dept. of Neurology and Psychiatry, General Hospital, 4020 Linz. Austria gerhard.ransmayr@akh.linz.at

Amyotrophic lateral sclerosis (ALS) is a progressive, neurodegenerative disease characterized by loss of motor neurons in the cerebral cortex, brain stem, and spinal cord. A minority of ALS patients $(5-10 \%)$ have a familial form (FALS) and $20 \%$ of these demonstrate mutations in the $\mathrm{Cu} / \mathrm{Zn}$ SOD 1 gene. Mice expressing the human mutant SOD1 gene develop age-dependent ALS-like neurological symptoms. We studies the mRNA expression profile in post-mortem spinal cord sections from sporadic ALS patients and human controls Using cDNA microarray gene expression we found significant increases in mRNA of cathepsin B (484\%) and cathepsin D (206\%), which are cysteine proteases that mediate intracellular protein turnover in the lysosme. MRNA of apolipoprotein E which is closely associated with the pathogenesis of neurodegenerative diseases, was also markedly increased $(290 \%)$. Further analysis with specific probes revealed that the expression of these genes also increases in hSOD1-G93A transgenic mice. They show enhancement with disease progression and peak at the end stage of the illness. Our data from ALS patients supported by findings using the transgenic mouse model indicates a crucial role of apolipoprotein- $E$ and cathepsin B/D in the pathogenesis of sporadic and familial forms of ALS.

Keywords: amyotrophic lateral sclerosis (ALS), mutant $\mathrm{Cu} / \mathrm{Zn}$ SOD1 gene, cDNA microarray, apolipoprotein E, cathepsin

$\mathrm{B} / \mathrm{D}$

Neuroprotection by a dextrocannabinoid in a 3 hours therapeutic window assessed functionally in transient MCA occlusion in rats

Bar-Joseph A., Lavie V., Berckovitch Y., Azulay M., Weksler A., Grienstein Y., Garzon A., Greenberg O., Elgamiel R., Amselem S. and Fink $G$.

Pharmos Ltd, Kiryat Weizmann, Rehovot 76326

The nonpsychotropic dextrocannabinoid, PRS-211,092, a novel analog of Dexanabinol, does not bind to the NMDA receptor but has powerful anti-inflammatory properties (in-vitro). PRS-211,092 inhibits the ischemia-induced increased gene expression of cyclooxygenase-2 (COX-2) and proinflammatory cytokines and chemokines in brain of transient middle cerebral artery occluded (MCAo) animals. The aim of the present study was to determine whether PRS-211,092 had long-term beneficial effects on functional following focal brain ischemia. The MCA of Sprague Dawley male rats was occluded for 120 minutes by intraluminal suture under halothane anesthesia. PRS-211,092 $5 \mathrm{mg} / \mathrm{kg}$ iv or PEG/ethanol vehicle alone were administered 1 hour after the end of the ischemic insult. The neuroprotective efficacy of the compound was evaluated by the "staircase test" (Montoya et al - J. Neurosci. Meth. 36:219-228 1991). Rats were trained for one week before MCAo, twice a day for $15 \mathrm{~min}$. in each test. Staircase test performance was determined immediately before and at approximately 6-day intervals after MCAo for a period of about 3 weeks. PRS-211,092 improved the staircase test performance on the contralateral side by $61 \%$ compared with that in animals treated with vehicle alone $(p<0.05)$. Thus, PRS-211,092 is an effective neuroprotectant in transient focal brain ischemia, even when administered 3-hours after the start of MCAO. These data, together with the fact that PRS-211,092 shows no toxicity suggest that this dextrocannabinioid may be worth considering for the treatment of stroke in man.

Keywords: stroke, staircase test, neuroprotection, MCAo

\section{Novel cannabinoids are analgesics in noxious and} neuropathic pain models

Bar-Joseph A., Richstein A., Dar D.E., Garzon A., Nudelman A., Menashe N., Amselem S. and Fink G.

Pharmos Ltd, Kiryat Weizmann, Rehovot 76326

Cannabinoid receptor agonists inhibit responses to painful stimuli by activation of the CB1 receptor, located mainly in the CNS, and the $\mathrm{CB} 2$ receptor, expressed mainly by inflammatory and immune cells. We have investigated the possible analgesic effect of novel, nonpsychotropic, cannabinoids, PRS-211,096 and PRS-211,335, which have a selective affinity for the CB2 (IC50 for both compounds = $\operatorname{lnM}$ ) compared with the CBI receptor (IC50 of 28 and $87 \mathrm{nM}$, respectively). Both compounds, administered IP, were effective analgesics for 
nociceptive pain as assessed by tail flick test in ICR male mice. PRS-211,096 demonstrated dose-dependent (2 to $10 \mathrm{mg} / \mathrm{kg}$ ) analgesia, which at $10 \mathrm{mg} / \mathrm{kg}$, was similar to that of morphine $(5 \mathrm{mg} / \mathrm{kg}) 30 \mathrm{~min}$ after drug injection. In animals treated with PRS-211,096, significant analgesia was still evident at $90 \mathrm{~min}$ (by which time morphine analgesia was no longer detectable), and at $150 \mathrm{~min}$ after injection. PRS-211,335 also induced significant analgesia at dosages of 2 and $4 \mathrm{mg} / \mathrm{kg}$ at $30 \mathrm{~min}$ after injection, a trend that was still evident at $90 \mathrm{~min}$ after injection.

PRS-211,096 was also tested for possible efficacy as an analgesic for neuropathic pain using the model of Bennett and Xie in the rat. Administered IP at doses of 0.5, 1.0 and 5.0 $\mathrm{mg} / \mathrm{kg}$, PRS-211,096 reduced tactile hyperalgesia, allodynia and thermal hyperalgesis in a dose-response manner. The efficacy of PRS-211,096 at $5.0 \mathrm{mg} / \mathrm{kg}$ was similar to that produced by morphine at a dosage of $5.0 \mathrm{mg} / \mathrm{kg}$. These cannabinoids may be novel and potent analgesics for the treatment of noxious and neuropathic pain in man.

Keywords: pain, neuropathic; cannabinoids

Di-isopropylfluorophosphate (DFP) on postnatal days 4-10 impairs passive avoidance in female, but not male adult mice

Ben-Bashat G. and Kofman O.

Dept. of Behavioral Sciences and Zlotowski Center for

Neuroscience, Ben-Gurion University of the Negev

Acetylcholinesterase inhibitors (AChE-I) are widely used as domestic and agricultural organophosphate pesticides, exposing a substantial population to the risk of neurological damage. ACh plays a major role in encoding, attention and regulation of cortical development. Sexual dimorphism has been found in cholinergic development and in behavioral deficits after treatment with AChE-I's. A single exposure to (AChE-I) in 3-10 day old mice caused down-regulation of muscarinic receptors, impaired spatial learning and altered motor behavior in females only (Dam et al. Developmental Brain Research 121:179-187 [2000]). The present study examined the effect of chronic developmental exposure to AChE-I on avoidance learning. Neonatal C57BL/65 mice of both sexes were injected with $1 \mathrm{mg} / \mathrm{kg}$ sc DFP or saline on postnatal days 4-10 and tested at age 4 months on the step-down passive avoidance paradigm. On Day 1, mice were placed individually on a vibrating platform above a metal grid floor. Upon stepping down, the mouse received a $0.5 \mathrm{~mA}$ footshock for 5 sec. The procedure was repeated until the mouse made 2 escape responses up to a maximum of 10 trials. The test phase, $24 \mathrm{hr}$ later, consisted of a single trial without footshock. Females administered DFP had shorter step down latencies than controls on the test day, whereas no difference was found between DFP and control males (sex $\mathrm{x}$ treatment $\mathrm{x}$ trial interaction, $\mathrm{F}_{2} \mathrm{~F}_{10}=4.30, \mathrm{p}<0.02$ ). No difference in pain thresholds was found between DFP and controls. The results suggest a greater sensitivity of females to deficits in fear-motivated learning, following early exposure to AChE-I's. Keywords: acetylcholine, passive avoidance, development

The $M_{1}$ and $M_{2}$ muscarinic $G$-protein-coupled receptors

Ben-Chaim Y. ${ }^{1}$, Tour O. ${ }^{1,2}$, Dascal N. ${ }^{3}$, Parnas I. ${ }^{1}$ and Parnas $\mathrm{H}$.

${ }^{1}$ The Otto Loewi Minerva Center for Cellular and Molecular Neurobiology, the Hebrew University, Jerusalem 91904;

${ }^{2}$ Dept. of Pharmacology, University of California San Diego, La Jolla, CA 92093-0647, USA, (at present); ${ }^{3}$ Dept. of Physiology and Pharmacology, Tel Aviv University, Tel- Aviv 69978

G-protein coupled receptors are not considered to exhibit voltage sensitivity. Here, using Xenopus oocytes, we showed that the muscarinic $M_{2}(\mathrm{~m} 2 \mathrm{R})$ and $\mathrm{M}_{1}(\mathrm{mlR})$ receptors, known to modulate neurotransmitter release, are voltage sensors. The m2R-mediated G-protein gated potassium channel currents were used to assay the activity of the $m 2 R$. We found that the apparent affinity of the $m 2 R$ toward acetylcholine $(A C h)$ was reduced upon depolarization. The endogenous $\mathrm{mlR}$ - mediated $\mathrm{Ca}^{2+}$ dependent chloride currents were used to assay the activity of the mlR. The mlR also shown exhibits voltage sensitivity but its apparent affinity toward $\mathrm{ACh}$ was increased upon depolarization. Direct binding experiments of $\left[{ }^{3} \mathrm{H}\right]-\mathrm{ACh}$ to individual oocytes expressing either $\mathrm{m} 2 \mathrm{R}$ or $\mathrm{mlR}$ confirmed the electrophysiological findings. The cumulative results indicate that membrane potential affects either the G-proteins coupled to the $m 2 R$ and the $m 1 R$ or the receptors themselves Based on the fact that G-proteins are peripheral membrane proteins and considering the large similarity between the different G-proteins coupled to the $m I R$ and the $m 2 R$, we favor the possibility that the $\mathrm{m} 1 \mathrm{R}$ and the $\mathrm{m} 2 \mathrm{R}$ are by themselves voltage sensors.

Keywords: G-protein coupled receptors, muscarinic receptor, voltage sensitivity, Xenopus oocytes

CuZnSOD deficient mice show late-phase improvement of neurobehavioral recovery after $\mathrm{CHI}$ and altered cortical reductive capacity

Beni SM ${ }^{1}$ Krool-Gal-ron $\mathrm{N}^{2}{ }^{2}$ Kohen $\mathrm{R}^{3}{ }^{3}$, Trembovler V. ${ }^{1}$, Shohami ${ }^{1}{ }^{1}$ and Barzillai $A^{2}{ }^{2}$

Depts. of ${ }^{1}$ pharmacology and ${ }^{3}$ pharmaceutics, the Hebrew University, Jerusalem; 'Dept. of Neurobiochemistry, Tel-Aviv University

Impaired redox homeostasis after brain trauma mediates cell-death pathways. CuZnSOD catalyzes $\mathrm{O}_{2}$ to $\mathrm{H}_{2} \mathrm{O}_{2}$ conversion, and along with other antioxidants constitute endogenous defense mechanisms. SOD administration was not beneficial in clinical trials, yet, SOD-transgenic mice display decreased lesion and apoptosis after brain trauma/ischemia. We assessed the effect of CuZnSOD deficiency on on outcome and reductive capacity after closed head injury (CHI). CHI was induced in CuZnSOD knockout (KO) and wild type (WT) mice, using a weight-drop devise. Neurobehavioral function was evaluated up to $14 \mathrm{~d}$ after CHI, using Neurological Severity Score (NSS). The difference between NSS at $\mathrm{lhr}$ post $\mathrm{CHI}$ and at later times ( $\triangle$ NSS) reflects recovery. Brain water content was measured $24 \mathrm{hr}$ after CHI. Reductive capacity was evaluated in sham (non-injured) and $5 \mathrm{~min}$ post-CHI mice using cyclic voltammetry. Sham KO mice displayed greater failure to exit a circle $(p<0.0001)$ and to balance over a beam $(p<0.005)$ After CHI water content increased in the injured cortex $(p<0.05)$ but to a similar extent in $\mathrm{KO}$ and WT. Yet, unexpectedly, at $14 \mathrm{~d}$ after $\mathrm{CHI}$, KO mice showed greater recovery $(\triangle$ NSS 3 vs $1 ; \mathrm{p}<0.02)$. Cortical reductive capacity was higher in sham KO compared to WT $(p<0.05)$ and decreased at $5 \mathrm{~min}$ post $\mathrm{CHI}(\mathrm{p}<0.05)$, whereas no changes occured in the WT. Hepatic reductive capacity was also higher in sham KO $(\mathrm{p}<0.0001)$ but was not affected by $\mathrm{CHI}$, in contrast to WT in which it increased $(\mathrm{p}<0.01)$. We propose that $\mathrm{KO}$ mice displayed late-phase better recovery after $\mathrm{CHI}$ which is not accounted for by the early post CHI alterations in reductive capacity.

Keywords: traumatic brain injury, oxidative stress, super oxide dismutase (SOD), antioxidants

The dose response effect of noladin ether on diet restricted mice

Ben Menachem A. ${ }^{1}$, Okon A. ${ }^{1}$, Avraham Y. ${ }^{1}$, Zlotariev O. ${ }^{1}$, Abel N. , Mechoulam R. and Berry EM.

Depts. of Human Nutrition and Metabolism ${ }^{l}$ and of Natural Products ${ }^{2}$, Hadassah Medical School, the Hebrew University.

Jerusalem 91120

Noladin ether ( $\mathrm{Nol})$ was isolated from porcine brain and identified as an endocannabinoid binding to $\mathrm{CB} 1$ cannabinoid receptor.

Aims: To evaluate the effect of noladin ether in mice under diet restriction (DR) as possible treatment for Anorexia Nervosa

Methods: Female Sabra mice assigned to 4 groups were fed on 2.5 hours a day diet for 14 days. Correspondingly were administrated i.p. different Nol treatment (Vehicle, nol 0.001 $0.01,0.1 \mathrm{mg} / \mathrm{kg}$ ). Food consumption (FC) and weight were measured. Cognitive function was evaluated using 8-arm maze and activity by $x / y$ beam apparatus. After 14 days $D R$ all groups were fed ad-libitum

Results: FC of mice administered $0.001 \mathrm{mg} / \mathrm{kg} / \mathrm{day}$ Nol was significantly higher than those received vehicle $(\mathrm{p}<0.0001)$. However, 0.01 and 0.1 caused significantly lower consumption $(\mathrm{p}<0.0001,<0.02$ respectively)

Cognitive function: $0.1 \mathrm{Nol}$ showed a significant decline in maze performance vs vehicle 0.001 and $0.01 \mathrm{nol} .(\mathrm{p}<0.05)$.

Activity: 0.001 and 0.1 nol. attained significantly higher beam apparatus performance than control. $(p<0.005)$

Weight: No significant weight differences were obtained after one month of trial. Nevertheless, all mice groups lost an average of $10 \%$ in their weight

Mice on diet restriction under noladin treatment $(0.001 \mathrm{mg} / \mathrm{kg})$ increased their food intake while their activity was significantly higher than control and their weight didn't differ from vehicle. FC of mice under noladin $(0.1 \mathrm{mg} / \mathrm{kg})$ decreased while their activity increased whereas their weight didn't differ probably due to decrease in BMR through IP3 signaling system(Leptin and endocannabinoids)

$\mathrm{A}$ bi-phasic dose response in the FC (nol $0.001>$ vehicle $>0$. 1 $\mathrm{mg} / \mathrm{kg}$ ) was noted. Cognitive function of vehicle, nol 0.001 , nol 0.01 was improved, whereas on nol 0.1 it declined 
Noladin has significant effect on food consumption and activity. Thus, low doses of noladin may possibly enhance appetite in patients with Anorexia Nervosa and weight loss such as cancer and Aids cachexia. No such treatment is yet available.

Keywords: Noladin, endocannabinoids, diet restriction, anorexia, food consumption, activity

\section{Visual and auditory "retain-and-compare" deficits in} dyslexia

Ben-Yehudah G. ${ }^{1}$ and Ahissar M. ${ }^{2,3}$

Depts. of Neurobiology ${ }^{I}$, Psychology ${ }^{2}$ and ${ }^{3}$ Center for Neural Computation, Hebrew Universityof Jerusalem, Jerusalem

Previously, we found that dyslexics' contrast detection of temporally modulated gratings was impaired only when they were required to "retain-and-compare" between sequentially presented stimuli (Ben-Yehudah et al., Brain 124: 1381-95 [2001]). We now ask whether similar deficits characterize the performance of dyslexic adults when the stimuli are stationary. Using both auditory (tones) and visual (sinusoidal gratings) stimuli, we found that dyslexics' sequential frequency discrimination is significantly impaired in both modalities. We further compared visual frequency discrimination under two conditions. In the simultaneous condition, observers were asked whether the stripes were denser in the upper or lower half of the screen. In the sequential condition, observers compared stripe density between two successive intervals. Consistent with our previous findings, the majority of the dyslexic group (74\%) was significantly impaired on the sequential condition. A significantly smaller group effect was found for the simultaneous condition. Classifying dyslexic participants according to their auditory frequency discrimination yielded two subgroups with different visual performance. The dyslexic subgroup with poor auditory abilities (mean JND $21.8 \%$ ) was impaired under both visual conditions, whereas the other dyslexic subgroup (mean JND $3.4 \%$ ) was impaired only under sequential presentation. These results imply that dyslexics with poor psychoacoustic performance suffer from broad visual and auditory perceptual impairments. The perceptual deficits of dyslexics with good psychoacoustic performance are revealed only when the task requires retaining and comparing subsequent visual stimuli. One explanation for dyslexics robust retain-and-compare deficit is impaired memory. Dissociating between deficient lower-level perceptual memory and higher-level working memory will be the focus of future studies.

Keywords: dyslexia, memory, frequency discrimination

\section{Isoform specific effects of apolipoprotein $\mathrm{E}$ on lipid uptake} by neuroblastoma $\mathrm{N} 2$ a cells

Berger L., Dvir A. and Michaelson D.M

Dept. of Neurobiochemistry, Faculty of Life Sciences, Tel Aviv University, Tel Aviv 69978

Apolipoprotein $\mathrm{E}$ (apoE), is the major lipoprotein of the brain. There are three major apoE isoforms (apoE2, apoE3 and apoE4) of which apoE4 is a major risk factor of Alzheimer's disease. In the present study we investigated the possibility that apoE affects neuronal lipid metabolism isoform specifically. This was performed by measurements of the effects of apoE 3 and apoE4 on the incorporation of $\left[{ }^{14} \mathrm{C}\right]$ acetate into lipids of neuroblastoma N2a cells and on the uptake of exogenous lipids. Addition of apoE3 and apoE4 $(30 \mu \mathrm{g} / \mathrm{ml})$, either in the presence or the absence of the exogenous lipid source Intralipid, resulted in partial inhibition of the incorporation of $\left[{ }^{14} \mathrm{Clacetate}\right.$ into neuronal lipids whose extent was similar for both isoforms. However, the incorporation of $\left[{ }^{14} \mathrm{C}\right]$ acetate into cells which were treated with the cholestcrol inhibitor Lovastatin and with Intralipid was partially inhibited by apoE3 and was unaffected by apoE4. The difference however was not statistically significant. In contrast the effects of apoE on the uptake of exogenous lipids, as measured by the incorporation of linoleic acid (C18:2) into cellular phospholipids, was markedly enhanced by apoE3 but not by apoE4. These findings show that the uptake of exogenous lipids into neuroblastoma $\mathrm{N} 2 \mathrm{a}$ is enhanced isoform specifically by apoE3 and suggests that the pathological effects of apoE4 in vivo may be mediated by a similar mechanism.

Keywords: apolipoprotein E, lipids, neuroblastoma
Prophylactic effects of phenytoin in bipolar illness: a controlled study

Bersudsky Y., Mishory A., Levine J., Winokur M. and Belmaker RH.

Stanley Foundation Research Center, Beer-Sheva Mental Health Center, Ben Gurion University of the Negev,

\section{Beer-Sheva}

Phenytoin, a classical anticonvulsant, shares the property of blockade of voltage activated sodium channels with antimanic anticonvulsants. We therefore planned a trial of phenytoin in acute mania. Thirty-nine patients entered a five week double-blind controlled trial of haloperidol plus phenytoin vs haloperidol plus placebo; thirty patients completed at least three weeks; twenty-five completed five weeks. Significantly more improvement was observed in those patients receiving phenytoin. We then planned a prophylactic study of phenytoin in bipolar disorder. Patients entered the study if they had been out of hospital for at least one month and had inadequate prophylaxis in the past on lithium, carbamazepine or valproate, and had at least one episode per year for the previous two years. Ongoing prophylactic treatment was not changed (lithium, carbamazepine, valproate or neuroleptic). Ratings (BPRS, YMS, HDS, GAS) were done by the clinical treating psychiatrist at baseline and once monthly thereafter. After six months patients were crossed over during a month of weekly visits with one drug (phenytoin or placebo) being reduced by $100 \mathrm{mg}$ weekly and the other increased by $100 \mathrm{mg}$ weekly. Twenty seven observation periods (six-month phases) were studied for 20 patients. Kaplan-Meier survival analysis showed highly significant benefits for phenytoin addition. Blockade of voltage-activated sodium channels may be a common therapeutic mechanism of many anticonvulsants in mania, and phenytoin may be a therapeutic option for some manic patients

Keywords: phenytoin, bipolar disorder, anticonvulsants

Dopamine, through direct interaction with its receptors, triggers IL-10 and TNF $\alpha$ secretion from normal, cancer and antigen-specific human T-cells

Besser $M^{l}$ and Levite $M^{1,2}$

The Sackler Faculty of Medicine, Tel-Aviv University, Tel Aviv 69978; ${ }^{2}$ Dept. of Neurobiology, Weizmann Institute of Science, Rehovot 76100

Under physiological conditions, T-cells may encounter dopamine in brain, blood, and various peripheral-organs. Moreover, dopamine is elevated in the plasma in certain conditions of uncoping-stress, and injected iv in several emergency conditions. Can dopamine directly trigger T-cell function?

We recently discovered that dopamine interacts directly with its D3 and D2 receptors on normal human T-cells, activates $\beta 1$-integrins, and induces subsequent T-cell adhesion.

Can dopamine also drive T-cells into cytokine secretion?

To challenge this question we applied dopamine or dopaminergic D1-D4 receptor agonists to normal peripheral, cancer, and antigen-specific human T-cell clones (of Th0, Thl, and Th 2 phenotype), and analyzed the secretion of IFN $\gamma$, TNF $\alpha$, IL-4 and IL-10.

We found that dopamine, in the absence of any additional molecule, can induce significant cytokine secretion from different $\mathrm{T}$-cell types. The most prominent effect was the triggering of $\mathrm{LL}-10$ secretion, a Th2- characteristic anti-inflammatory and suppressive cytokine. Dopamine also triggered a marked secretion of TNFa, a potent pro-inflammatory Thl-associated cytokine, playing a crucial role in various inflammatory-diseases.

Dopamine's effects were mimicked by specific dopaminergic-agonists, and blocked by the respective antagonists. Interestingly, the results suggested that dopamine may trigger IL-10 and TNF $\alpha$ secretion via different receptor subtypes.

Taken together, we speculate an active role for dopamine in various IL-10 and TNF $\alpha$-dependent T-cell activities. Dopamine's effects may be either beneficial or detrimental, depending on the context.

We further speculate dynamic dopamine-T cell interactions in patients with schizophrenia, Parkinson's disease, Alzheimer disease and migraine, where modulations in dopamine-receptor levels in peripheral blood lymphocytes were recently reported Keywords: dopamine, T-cells, cytokines 
Self organized mapping of spontaneous activity in cat visual cortex

Bibitchkov D., Kenet T., Tsodyks M., Grinvald A. and Arieli A. Dept. of Neurobiology, Weizmann Institute of Science, Rehovot 76100

Optical imaging recordings of the primary visual cortex of anesthetized cats using voltage sensitive dyes show that activity patterns with significant correlations with orientation preference maps arise spontaneously at least $20 \%$ of time. We found that ongoing activity is composed of a set of states, that switch dynamically. We applied a self-organized mapping algorithm (Kohonen, Self-organizing maps [1997]) to determine the topology of these states and to study the dynamics in a genuinely independent way. The results confirm that states of spontaneous activity can be mapped onto a one-dimensional ring structure of templates similar to single condition orientation maps. Long epochs of smooth transitions between neighbouring orientations were obviously present in the data. The duration of such sequences was much longer than typical temporal correlation times. We also calculated the typical speed of transition and typical times spent in certain states of spontaneous activity. We found a clear preference of ongoing activity for states correlated with single condition maps of cardinal orientations. The results show that the ongoing activity is tightly linked to its functional architecture. keywords: visual cortex, Kohonen map, orientation preference

\section{Sex differences in brain activation: a multiple-modality} fMRI study

Biegon A. ${ }^{1}$, Harrington G. $^{2}$, Girshick AR $^{1}$, Pareto D. ${ }^{3}$, Klein $\mathrm{G}^{1}$ and Buonocore $\mathrm{M}$

${ }^{I}$ Dept. of Functional Imaging, LBNL, Berkeley, CA, USA;

${ }^{2}$ Dept. of Radiology, UC Davis Med. Center, Sacramento CA; ${ }^{3}$ Unitat Biofisica, Univ. Barcelona, SPAIN

Recently identified sex differences in the outcome of severe head injury have prompted us to use fMRI to investigate sex differences in volumes of activation in healthy men and women performing motor, sensory, visual, auditory and language tasks. The studies were carried out on a 1.5T GE LX MRI system using adaptations of a published presurgical procedure (Hirsh et al. Neurosurgery 47:711-722 [2000]). Male and female volunteers were paired for age (range 19 to 60 ) and scanned while clenching the fist of the dominant or non dominant hand, then they viewed a flashing checkerboard while an experimenter rubbed the inside of the palm and fingers with a rough (velcro) surface. Picture naming and verb generation (from spoken words) were used as concomitant language/visual and language/auditory stimuli, respectively. Significant $(\mathrm{p}=0.0001+$ cluster $>=2)$ activations were identified using AFNI software (Cox RW, Comput. Biomed. Res. 29:162-173 [1996]). Next, large regions of interest (ROIs) were drawn bilaterally on the anatomical slices corresponding to the known location of primary motor, sensory, visual and auditory cortices as well as Broca's area and the number of activated voxels in each ROI was counted. Among the 10 subjects scanned and analaysed so far (mean age 29 for women and 30 for men), the verb creation task resulted in a robust and lateralized (left $>$ right) activation in both men and women. The activation volume in women was 3-4 fold the volume in men, possibly contributing to the relatively high proportion of good neurological recovery among female survivors of head injury.

Keywords: imaging, language, brain injury, male, female

Time dependent changes in NMDA receptor binding after closed head injury in mice

Biegon A. ${ }^{1}$, Fry P.A.., Paden C.M. ${ }^{2}$, Alexandrovich A. ${ }^{3}$ and Shohami E.

${ }^{I}$ Dept of Functional Imaging, LBNL, Berkeley, CA, USA

${ }^{2}$ Dept. of Cell Biology and Neuroscience, Montana State University, Bozeman, MT, USA; ${ }^{3}$ Dept of Pharmacology, Hebrew University. Jerusalem

Traumatic brain injury (TBI) is associated with an early, large and transient increase in glutamate release. The purpose of this study was to follow the time course of changes in NMDA receptor (NMDAR) function post TBI by quantitative autoradiography with the use-dependent antagonist $\left[{ }^{3} \mathrm{H}\right] \mathrm{MK} 801$. Brains of injured (4-5/group) and sham treated mice were removed at 15 minutes, $1,4,8$ and 24 hours, or 7 days following injury. In vitro autoradiography was performed as previously described (Biegon et. al. J. Neurochem. 82:1-11 [2002]). We found that at the level of impact closed head injury was associated with a progressive, $40-80 \%$ decrease in activated NMDA receptors bilaterally, which peaked at $8 \mathrm{hrs}$ post injury and persisted up to the last experimental time point ( 7 days). At 7 days, there was a larger deficit of receptors in the injured hemisphere. This late decrease was limited to regions at close proximity to the impact and was clearly associated with neuroinflammation and neuronal loss. At levels posterior to the impact, there was a transient but substantial $(>50 \%)$ increase in NMDA receptor binding at 15 minutes followed by a sustained decrease. In conclusion. NMDA receptor activation after TBI is short lived $(<1 \mathrm{hr})$ and is followed by a profound and long lasting suppression of receptor function. These findings may explain the short $(<1 \mathrm{hr})$ therapeutic window of NMDA antagonists in animal models of TBI; their failure in the clinic; as well as some of the most common clinical manifestations of severe and moderate head injury.

Keywords: brain injury, glutamate, autoradiography

Use of F-18 FES for estrogn receptor (ER) imaging in rat and human brain

Biegon A. ${ }^{1}$, Alvarado M.. ${ }^{1}$, Budinger T.F. ${ }^{1}$, Mankoff DA. ${ }^{2}$ $\mathrm{O}^{\prime}$ Neil JP. ${ }^{1}$ and VanBrocklin $\mathrm{HF}^{1}$

${ }^{1}$ Dept of Functional Imaging, LBNL, Berkeley, CA, USA ${ }^{2}$ Dept of Radiology, Washington U. Seattle, WA, USA

Estrogens or antiestrogens are currently used by millions of women, but the interaction of these hormonal agents with brain ER has not been charaterized to date. FES (F-18 labeled fluoroestradiol) has been used successfully for PET imaging of ER in the breast. The utility of this tracer for brain ER imaging is being investigated in rats and humans. Ovariectomized (OVX) rats were implanted with pellets containing estradiol, tamoxifen or blank pellets. Three weeks later, groups of 4 animals were injected IV with FES $(0.5 \mathrm{mCi} / \mathrm{Kg})$ and killed 5 , 20 or 60 minutes later. Brain samples were dissected and counted. As expected, the rank order of tracer uptake was hypothalamus, preoptic area, amygdala, cortex, and cerebellum. OVX controls had a high $(3.7+1-0.38)$ hypothalamus / cerebellum binding ratio at $60 \mathrm{~min}$. Estradiol and tamoxifen resulted in a significant reduction of this ratio to $1.8+/-0.08$ and $2.1+/-0.27$, with $p=0.001$ or 0.003 compared to OVX, respectively. Cortical ER were similarly occupied by estradiol and tamoxifen ( $\mathrm{p}=0.01$ and 0.04 , respectively compared to OVX). In pilot human studies, we aquired PET brain images (single 10 minutes acquisition approximately 2 hrs after tracer injection of 4-6 $\mathrm{mCi}$ FES) in 6 patients undergoing FES PET as part part of an ongoing breast cancer research protocol. Increased tracer uptake was observed in the preoptic/hypothalamic region relative to other gray matter regions, suggesting this tracer can be used for non-invasive imaging of human brain ER by PET

Supported by DOD DAMD17-01-1-0289(AB), NIH AG05890(TFB) and NIH CA42045(DAM)

Keywords: PET, SERMs, tamoxifen

Source of knowledge of category affects cortical activity during classification of geometric figures: a visual ERP study

Bigman $\mathrm{Z}$., and Pratt $\mathrm{H}$

Evoked Potentials Laboratory, Technion, Israel Institute of

Technology, Haifa

Categorization strategies can include abstraction and application of a stated explicit rule, or judgement of similarity to other category members. In order to determine the brain activity associated with these strategies of classifying a new stimulus, ten 11-13 year old subjects participated in two tasks of classification of a visually presented geometric figure: (1) classification by a stated category - target feature identification and (2) classification by a category induced by a previously presented series of two figures belonging to the category. Figures varied in size, color and shape and Event-Related Potentials (ERPs) to the stimulus being classified were recorded.

ANOVA showed P220 (attributed to strategy selection) amplitudes were larger when elicited in classification by induced category, and larger N300 (related to stimulus mismatch) was noted in response to classification by stated rule. Principal Component Analysis showed multiple sources of activity at occitipal sites in response to classification by stated rule; and both frontal and parietal activity in response to classification by induced category.

These results suggest less complex cortical activity during classification by a stated rule than by a previously induced category, involving different brain structures and imply use of different classification strategies according to the source of knowledge of the category. Results support the theory that when possible, categorization of a new stimulus is based on judgement of similarity to other category members, and not application of a rule

Keywords: vision, ERP, P200, N300, categorization 
Presynaptic 2A-type receptors mediate the effect of serotonin on inhibitory GABAergic transmission in layer 4 of developing mouse barrel cortex

Binshtok A.M., Fleidervish I.A. and Gutnick M.J. Koret School of Veterinary Medicine, The Hebrew University of Jerusalem, Rehovot 76100

We previously demonstrated that during early stages of barrel formation in mouse somatosensory cortex, brief applications of 5 -HT in Layer 4 induce complete and reversible blockade of stimulus-elicited IPSCs. We now show that this effect is mediated by a presynaptic 5-HT2A receptor. In tangential slices of Layer 4 from P5-P14 mice, puff applied 5-HT (2mM) reduced the frequency of spontaneous IPSCs by blocking high-amplitude, action potential-dependent events. Application of 5-HT did not affect responses elicited by direct GABA application. The blockade of 5-HT2A receptors with ketanserine tatrate $(1 \mu \mathrm{M}$ puff application, or $20 \mu \mathrm{M}$ bath application) prevented this 5-HT effect on GABAergic transmission. By contrast, application of antagonists to other 5 -HT receptors (1A - WAY-100635; 1B - isamoltane hemifumarate; 1D - BRL 15572; 2B/2C - SB 206553; 5-HT3 LY-278,584 malealate) was ineffective in all cases. In field potential recordings in Layer 4 slices from animals younger than P14, bath application of 5-HT $(200 \mu \mathrm{M})$ reversibly induced all-or-none epileptiform discharges that were similar to those induced by GABAA blockers. This effect was not seen in coronal slices, in which cortical circuit activity is dominated by neurons of other layers. It seems possible that during the first two weeks of life, during the time window when 5-HT becomes concentrated in presynaptic thalamocortical terminals, this modulator is co-released from these terminals along with glutamate, and that afferent activity thereby causes local disinhibition and consequent bursts of synchronous focal discharge. Such a mechanism might play a role in local circuit formation

Supported by grant from the Israel Science Foundation.

Keywords: barrel cortex; development; serotonin; IPSCs

\section{Changes in the extracellular space of rat spinal cord induced by glutamate revealed by diffusion MR
Biton I.E. ${ }^{1}$, Mayk A. ${ }^{2}$ Assaf $\mathrm{Y}^{1,3}$ and Cohen $\mathrm{Y}^{1}$ \\ ${ }^{7}$ School of Chemistry, Tel Aviv University, Tel Aviv 69978 ${ }^{2}$ TEVA Pharmaceutical Industries Ltd. and Sackler School of Medicine, Tel Aviv University, Tel Aviv 69978, ${ }^{3}$ The Wohl Institute for Advanced Imaging, Dept. of Radiology, Tel Aviv Sourasky Medical Center, Tel Aviv 64239}

The size and the geometry of the extracellular space (ECS) influence the diffusion of neuroactive substance. It has been shown that release of neurotoxic levels of glutamate into the ECS, which takes place in ischemia, trauma, and hypoxia, effects the ECS. In this study we have used diffusion MRS of deuterated tetramethyl ammonium chloride (TMA- $\mathrm{d}_{12}$ ), which is restricted to the ECS, to study the diffusion characteristics of the ECS before and after application of glutamate.

The study was performed on rat spinal cords, which were excised and immersed in $0.1 \mathrm{M}$ solution of TMA- $\mathrm{d}_{12}$ for 3 hours. After the MRS measurements the spinal cord was immersed in a saline solution containing L-Glutamic acid $(0.01 \mathrm{M})$ for 2 hours. MRS diffusion experiments were performed on an 8.4T NMR spectrometer using the stimulated echo (STE) diffusion sequence and with the following $\mathrm{G}_{\max }=160$ gauss $/ \mathrm{cm}$. The diffusion times were $50,150,250$ and $500 \mathrm{~ms}$. Diffusion was measured perpendicular to the long axis of the spine.

In these diffusion experiments two different diffusing components of TMA- $\mathrm{d}_{12}$, were found. The fast component $\left(D_{\text {fast }}=2.95 \pm 0.17 \times 10^{-4} \mathrm{~mm}^{2} / \mathrm{sec}\right)$ is relatively isotropic and has a tortuosity of $1.93 \pm 0.05$ and $2.18 \pm 0.09$ at diffusion time of $50 \mathrm{~ms}$ before and after application of glutamate, respectively. The slow diffusing component $\left(D_{\text {slow }}=1.30 \pm 0.01 \times 10^{-5} \mathrm{~mm}^{2} / \mathrm{sec}\right)$ is more anisotropic and has a tortuosity of $9.18 \pm 0.01$ and $9.91 \pm 0.39$ before and after application of glutamate, respectively. The tortuosity was found to depend on the diffusing time. The fast diffusing component observed in the MRS experiments show the similar characteristics found by iontophoretic method using selective electrode. All these observations are line with the cell swelling caused by the glutamate.

Keywords: diffusion MRS, extracellular space (ECS),

tetramethyl ammonium chloride (TMA- $\left.\mathrm{d}_{12}\right)$
Stimulus to feeling gap in the brain: the interaction of stimulus presentation mode and emotional appraisal Bleich M. ${ }^{1,2}$, Pianka P. ${ }^{1,2}$, Mintz M. ${ }^{2}$, Rotshtein P. ${ }^{1}$, Andelman $\mathrm{F}^{1}$ and Hendler T.

${ }^{1}$ Tel Aviv Sourasky Medical Center, Tel Aviv; ${ }^{2}$ Tel Aviv

University, Tel-Aviv, The Wohl Institute for Advanced Imaging, Tel-Aviv Sourasky Medical Center

Introduction: What bridges the gap between stimulus and feeling? Originally James claimed that this gap is filled merely by a feedback from an arousal response, while later others introduced the concept of cognitive assessment and appraisal of that arousal (1-3). These processes could well be complementary and may depend on the brain region and presentation mode of the stimulus. In this study we examined the interactions between the presentation mode of stimuli and their negative emotional appraisal in the brain by fMRI.

Methods: Eight right-handed female subjects were scanned in a 1.5T GE scanner. Experimental block paradigm consisted of two factors: stimulus type (pictures, faces, sentences) and stimulus assessment (naming of gender versus feeling). All stimuli were of negative valence, presented for $500-1500 \mathrm{msec}$ at a rate of $0.5 \mathrm{~Hz}$. Data analysis was preformed using BrainVoyager (Brain Innovation Co). Parametric maps were obtained by General Linear Model (GLM) for individual subject analyses and group analysis. Regions of interest included: extra striate cortex, amygdala complex and superior temporal region (ST).

Results: Visual cortex and amygdala responded significantly more to pictures than faces and sentences (main effect of stimulus, $p<0.001$ ). In contrary, STS responded significantly more to sentences than to pictures and faces (main effect of stimulus, $\mathrm{p}<0.05)$ more so for the left hemisphere (2-way interaction of laterality and stimuli type, $p<0.05$ ). Amygdala activation did not distinguish between emotional appraisal and gender identification, while visual and language regions were activated more by the emotional appraisal task (main effect of task: visual cortex-p<0.05; ST-p<0.07). Furthermore, in the visual cortex task effect was most prominent for pictures (2-way interaction, $\mathrm{p}<0.005$ ), while in the ST task effect was lateralized to the left hemisphere (planned comparison, $\mathrm{p}<0.05$ ).

Discussion: Stimuli affected activation level in sensory, limbic and language regions depending on the mode or presentation. In contrary, emotional appraisal effect was found only in sensory and language regions. These results suggest that the distinction between processes that link stimulus and feeling is reflected in differential activation of sensory, language and limbic regions. This difference depends on either mode of presentation and/or appraisal of negative emotional stimuli. References:

1. LeDoux J. (1986). The Emotional Brain. (New York: Touchstone)

2. James W. (1884). What is an emotion? Mind 9, 188-205.

3. Schachter S. and Singer J.E.(1962). Cognitive, social, and physiological determinants of emotional sate. Psychological Review 69, 379-399.

Keywords: emotions, amygdala, functional

Modulation of acetylcholinesterase in exercised rat muscles Blotnick E., Sharon S. and Anglister L.

Dept. of Anatomy and Cell Biology, Hadassah Medical School, Hebrew University of Jerusalem, Jerusalem 91120

Slow twitch muscles are readily recruited and are active continuously while fast-twitch muscles are recruited when increased motor effort is needed. The synaptic activity of muscles involves acetylcholinesterase (AChE) which is concentrated at the neuromuscular junctions and determines the duration of the neurotransmission activating the muscles Because synaptic AChE depends on neuromuscular activity we examined the regulation of $\mathrm{AChE}$ in fast and slow-twitch rat muscles following strenuous exercise. Rats were trained by walking on motor-driven treadmill $(10 \mathrm{~d}, 1 \mathrm{~h} / \mathrm{d}$ at a speed of $9 \mathrm{~m} / \mathrm{min}$ with $2 \mathrm{~min}$ sprints of $17 \mathrm{~m} / \mathrm{min}$, every $10 \mathrm{~min}$ ), so as to activate the fast-twitch leg muscles, not usually recruited in sedentary animals. Fast and slow-twitch leg muscles were isolated, AChE was extracted and its levels and isoform composition in trained and control-untrained muscles were analyzed. The results show increase in $\mathrm{AChE}$ content (per protein) in all examined fast-twitch muscles but not in the slow-twitch soleus muscle. The most significant increase was measured in trained gracilis anterior muscles (24\%). All muscles contained globular $\mathrm{G} 1+\mathrm{G} 2, \mathrm{G} 4$ and asymmetric A12-AChE isoforms (4-6S, $10 \mathrm{~S}$ and $16 \mathrm{~S}$ sedimentation values, respectively). While G1+G2 and A12-AChE activities in both fast and slow-twitch muscles remained unchanged after training, the tetramer, G4-AChE, increased significantly in the 
exercised fast-twitch muscles $(25-60 \%)$. These observations suggest that the G4-tetramer might become significant at the endplates of fast-twitch fibers following exercise. The density and distribution of this form at the endplates following exercise is examined.

Supported by Israel Sci. Found. 685/01 (LA).

Keywords: neuromuscular junction, fast twitch,

acetylcholinesterase, treadmill

A study of performance of heavy-load tasks of identification of geometric forms by naive subjects Blum B

Dept of Physiology \& Pharmacology, Tel Aviv University, Tel Aviv 69978

The goal of this study was to test the assumption that perceptual performance of tasks as of the present experiment is universally based on pre-prepared action schema; these derived from 'mental schema' idealized so as to fit category-wise, various on-coming individual test conditions. The study was a one repetition experiment, on subjects short in experience with the stimuli, the rationale being that this metes with "new situation" criteria. While one repetition is critical for the observer for statistical verification, to simulate realistically new situations, iterative presentations of 'part connectedness' stimuli were used. The results show load as impetus to shifting resources to earmarked higher demands, at cost of errors at easy tasks presumably in consequence of allotting these insufficient attention. High acuity demand, practically impossible for performance per se, is found enabled within context of the experiment. Eluciding this is $\mathrm{Ss}(\mathrm{P}<0.01)$ report that when having to judge between straightness and high acuity demand obliqueness they experienced on the former illusory obliquity in the opposite direction, thus presumably enhancing decision/ disambiguation. 'Paradigms junctures'- assumed points of across-mechanisms' joint probability interactions achieve enhanced performance on basis of degree of shared components' relevancy to a category relationship.

Keywords: mental schema; naive subjects; part connectedness paradigms; disambiguation

Intake and activation of the dorsal vagal complex in preweanling rats after preloods of corn-oil or mineral oil
Blumberg S. Torregrosşa A.-M. ${ }^{2}$, Smith G.P. ${ }^{2}$, Avnon Y.L. ${ }^{1}$, Shayit M. ${ }^{1}$ Hurwitz $\mathrm{I}^{3}$ and Weller A. ${ }^{1,3}$

${ }^{7}$ Dept. of Psychology and ${ }^{3}$ Interdisciplinary Program in Brain Science, Bar-Ilan University, Ramat Gan; ${ }^{2}$ Bourne Lab, Dept of Psychiatry, NY Presbytarian Hospital-Cornell University

Medical Coll, White Plains, NY, USA

Otsuka Long-Evans Tokushima Fatty (OLETF) rats lack cholecystokinin-A $\left(\mathrm{CCK}_{\mathrm{A}}\right)$ receptors. We have characterized an early postnatal emergence of hyperphagia and increased body-weight in infant OLETF vs. their controls, Long Evans Tokushima (LETO) rats, on postnatal days 2-4 and 9-11, during a 30-min independent ingestion test. In the current study we compared feeding suppression by $50 \%$ BW gastric preloads of corn oil and mineral oil in 18-20 days old OLETF and LETO pups, and in Long-Evans pups on postnatal days 9-11. $\mathrm{C}$-fos immunoreactivity was examined in area postrema (AP), the nucleus of the solitary tract (NTS), and in hypothalamic areas implicated in feeding regulation. LETO rats ingested significantly less after corn oil than after mineral oil preloads Their c-fos immunoreactivity in AP and NTS was increased compared to sham-preload controls. The effect of corn oil was not evident in OLETF pups; their mean number of $\mathrm{c}$-fos expressing cells was increased in the caudal, subpostremal, and intermediate NTS, and in the AP, but not as much as in LETO pups. Intake of Long-Evans pups was not lower after corn oil compared to mineral oil preloads, replicating previous ontogenetic findings in Sprague-Dawley rats (Weller et al. Physiol Behav 62:871-874 [1997]). Their activation patterns in the AP and NTS corresponded to the intake results. These findings provide support for the role of $\mathrm{CCK}_{\mathrm{A}}$ receptors in mediating intake-reduction early in ontogeny.

Supported by the US-Israel Binational Science Foundation

Keywords: CCK, c-fos, feeding, area postrema, nucleus of solitary tract

Differentiation and neurotransmitter phenotype acquisition in developing neurons - differential expression of genes and proteins

Bogoch Y., Tayar S., Bledi Y. and Linial M.

Dept. of Biological Sciences, Silberman Institute of Life

sciences, The Hebrew University, Jerusalem, Israel

P19 cells are embryonal carcinoma cells that serve as a model for studying differentiation processes including commitment to cell lineage. We have studied P19 cells following activation of neuronal differentiation. The potential of these cells to mature and efficiently release neurotransmitter (NT) was established in our lab. We discovered that several variables, most notably, cell density and various neurotrophic factors affects neuronal maturation, survival. and most surprisingly, the choice of NT phenotype. We have observed that NT phenotype acquisition in $\mathrm{P} 19$ cells is mediated by cell-cell contact and not by soluble factors. We have shown that changes in cell density are associated with changes in gene expression. A large-scale holistic view on gene expression was obtained by DNA chip technology. The data obtained from this screen was extensively analyzed with various bioinformatical methods. Among the genes found in that screen are members of the Wnt and Cullin families which function in transducing a signal from the cell surface.

Mass Spectroscopy comparative proteomics approach was applied on sparse and dense P19 induced cultures. Membranes were collected at different time windows following neuronal induction. Differentially expressing proteins were excised and sent to Mass-spectroscopy analysis. Currently, over 30 proteins were successfully analyzed by such methodology. Using 2D gels we were able to improve detection level and could identify relatively low expressing proteins. Most intriguing proteins are a variant of Drebrin - a putative dentritic-shaping molecule and several cytoskeletal-signaling molecules. Those and other proteins reflect the morphological reorganization that takes place while establishing P19 neuronal fate.

Keywords: neurotransmitter phenotype, neuronal

differentiation, p19 embryonal carcinoma

Photic resetting of the human circadian clock: applications to jet lag and shift work

Boivin D.B. and James F.O.

Douglas Hospital Research Centre, Dept. of Psychiatry, McGill University

Recent studies indicate that the endogenous circadian system is sensitive to much lower levels of light than initially suspected (Boivin DB et al Nature 1996;379:540-542.). It was also recently reported that $70 \%$ of the resetting effect of a bright light stimulus can be preserved even though it is interrupted by episodes of complete darkness for $63 \%$ of the time (Rimmer DW et al. Am J Physiol Regulatory Integrative Comp Physiol $2000: 279: \mathrm{R} 1574-\mathrm{R} 1579$ ). The usefulness of a judicious schedule of exposure to room light (first study) or to intermittent bright light (second study) to treat circadian maladaptation to jet lag or shift work was tested. In the first study, 15 healthy young men participated to a laboratory simulation of a Montreal to London voyage (Boivin DB and James FO. J Biol Rhythms 2002;17(3):266-276 ). They were exposed to 380 lux for 6 hours each day either before bedtime or in the morning. This study demonstrated that the schedule of exposure to room light can substantially affect circadian adaptation to a shifted sleep/wake schedule. In the second study, 15 nurses working permanent night schedules were studied for 3 consecutive weeks (in press). They were either exposed to bright light intermittently for 6 hours in their workplace (treatment group) or worked under their usua lighting conditions (control group). Circadian reentrainment was complete in the treatment group only. Results of these two studies underline the importance of the control of the overall pattern of light exposure in circadian adaptation to shifted sleep/wake schedules.

Motion induced blindness is affected by head-centered and object-centered mechanisms

Bonneh Y and Cooperman A

Dept. of Neurobiology, Brain Research, Weizmann Institute of Science, Rehovot 76100

In motion-induced-blindness (MIB; Bonneh et. al., Nature 411:798-801[2001]), a salient static pattern may disappear and reappear spontaneously in the presence of a global moving pattern. We have previously showed that this phenomenon is unlikely to reflect retinal suppression, sensory masking or adaptation, but the mechanisms involved in MIB are largely unknown. Recent evidence suggest that parietal mechanisms representing space in different reference frames may be involved in the control of awareness, and possibly in MIB. Here we ask what is the frame of reference in which disappearance during MIB occur. To answer this question we measured the magnitude of disappearance at different spatial locations in three different conditions: (1) retinotopic mapping in which a single target dot was presented at different locations relative to fixation, (2) a head-centered condition in which the direction of the head was displaced by $20 \mathrm{deg}$. to the right or left keeping direct fixation and (3) object-centered condition in 
which a target dot in fixed retinal location was surrounded by an elliptic contour with different relative displacement along it's main axis. Results show the effect of all three manipulations on disappearance. Anisometropic disappearance was found for all observers with more disappearance in the upper and upper-left visual fields. Disappearance map changed for different angles of head rotation even though the retinal locations were identical. The elliptic contour induced more disappearance around its focal points and less in its center. These results suggest that disappearance in MIB involves or is affected by head-centered and object-centered mechanisms Keywords: visual disappearance, visual awareness, visual space

Evaluating ion channel modulating activities in a Tarantula venom

Brass D., Dan P., Magnushevsky N., Marom A., Meidan O. and Meir A

Alomone Labs Ltd, P.O. Box 4287, Jerusalem 91042

The flow of ions through membrane channels is an essential component of cellular function. Recently, ion channels have emerged as potential targets for diagnostic and therapeutic agents due to their membrane localization and their contribution to the physiology and pathophysiology in many biological systems.

The venomous animal world offers a large variety of peptide toxins, with some exerting their biological activity on various ion channel activities, by means of high affinity interaction and selective modulation.

Alomone Labs R \& D focus on the discovery of new effective and specific ion channel peptide toxins. These toxins may serve as potential leader compounds for diagnostic and therapeutic agents, which affect various diseases, such as cancer, cardiovascular diseases and neurological disorders.

Alomone Labs has developed a technological platform for finding, characterizing, producing and modifying peptide-toxin ion channel modulators. It includes:

Separation and purification of peptide toxins from whole native venom.

Assays for finding molecularly defined ion channels as possible targets for the purified toxins, and for evaluating the characteristics of the toxin pharmacology.

Recombinant production and purification of a desired peptide toxin.

Here we focus on the separation and dissection of ion channel modulating activities found in the venom of one spider species. The spider Grammostola spatulata (Brown Morph Grammostola- BMG) venom effects on various cloned voltage dependent ion channels, was examined using two-electrode voltage clamped Xenopus oocytes.

The BMG whole venom strongly inhibited the following channels: $\mathrm{K}_{\mathrm{V}} 2.1, \mathrm{Na}_{\mathrm{v}} 1.5, \mathrm{Ca}_{\mathrm{v}} 1.2, \mathrm{Ca}_{\mathrm{v}} 2.2$ and $\mathrm{Ca}_{\mathrm{v}} 3.1$.

BMG venom was subjected to SP-sepharose fractionation, yielding 8 separate peaks and the blocking activity, within the venom was narrowed to specified peaks for each of the channels examined.

A peak containing single peptide that was identified by mass spectroscopy and amino-acid analysis as the previously described $\omega$-Grammotoxin SIA specifically blocked $\mathrm{Ca}_{\mathrm{v}} 2.2$ channels.

Strong blocking activity towards $\mathrm{K}_{\mathrm{v}} 2.1$ channels was found in two separate peaks that contain several peptides each.

One defined peak blocked $\mathrm{Na}_{\mathrm{v}} 1.5, \mathrm{Ca}_{\mathrm{v}} 1.2$ and $\mathrm{Ca}_{\mathrm{v}} 3.1$ However, further analysis showed that this peak contained several different peptides.

In conclusion, we show wide range and specific ion channel blocking activities, carried out by separated fractions of Tarantula spider venom. Some of these activities affect channels with poor specific pharmacology such as the $\mathrm{Ca}_{\mathrm{y}} 3.1$ channel.

Keywords: channels, toxin, Tarantula

Maternal deprivation increases alcohol consumption in adulthood in the rat

Brave M., Berger B.D. and Richter-Levin G.

Dep. of Psychology \& Brain and Behavior Research Center Haifa University, Haifa

Early maternal deprivation (MD) is considered an adverse stressful experience, associated with long-lasting effects in adulthood. It is suggested that this kind of stress may be a risk factor for the development of high alcohol consumption in adulthood

The aim of the present study was to examine, in a rat model, the relationship between early maternal deprivation, and subsequent development of high alcohol consumption. We further examined the influence of long-term isolation on MD modulation of alcohol consumption in adulthood

Method: Male Wistar rat pups were exposed to one of 4 different rearing conditions: 1) Maternal separation from PND 7 for 7 consecutive days, for one hour every day. 2) Long-term social isolation immediately post weaning. 3) Maternal deprivation and social isolation. 4) Regular rearing conditions. Pups were weaned at PND 28. At the age of 3 months all animals were examined in a free choice paradigm for alcohol and water consumption.

Results: We observed a significantly high rate of alcohol consumption only in rats that were maternally deprived 3 months before.

These finding indicate that MD has long lasting effects on emotional development.

In contrast to previous reports studying social isolation as a stressful experience, social isolation in this study was found to attenuate the effect the MD treatment. The main difference between the current and previous studies was that in the present study social isolation began immediately after weaning while in previous studies rats were exposed to social isolation in adulthood. Further examination is required to clarify these differences.

Supported by a grant from the Israel Foundation Trusties (2000) to $G$. $R-L$

Keywords: Maternal Deprevation; stress; alcohol

Location and size of dopaminergic and serotonergic cell populations are controlled by position of mid-hindbrain organizer

Brodski C ${ }^{1}$, Vogt Weisenhorn D.M. ${ }^{1}$, Signore M. $^{2}$, Sillaber I ${ }^{1}$, Oesterheld M. $^{1}$, Acampora D. ${ }^{2,3}$, Simeone A. ${ }^{2,3}$ and Wurst W. ${ }^{1}$ ' ${ }^{I}$ Max-Planck-Institute of Psychiatry, Kraepelinstr. 2-10. 80804-Munich and SF-Research Center, Institute for

Mammalian Genetics 85758 Oberschleißheim, Germany: ${ }^{2}$ MRC Centre for Developmental Neurobiology, King's College London, Guy's Campus, New Hunts House, London SE1 9RT,

UK; ${ }^{3}$ International Institute of Genetics and Biophysics, CNR, Via G. Marconi 12, 80125 Naples, Italy

Midbrain dopaminergic and hindbrain serotonergic neurons play an important role in the modulation of behaviour and are involved in a series of neuropsychiatric disorders. Despite the importance of these cells, little is known about the molecular mechanisms governing their development. During embryogenesis midbrain dopaminergic neurons are specified rostral to the mid-hindbrain organizer (MHO) and hindbrain serotonergic neurons caudal to it. We report that in transgenic mice in which the MHO is shifted caudally, the midbrain dopaminergic neuronal population expands to the ectopic positioned MHO and is enlarged. Complementarily, the extension of the hindbrain serotonergic cell group is decreased In adulthood these changes are preserved and the additional ectopic dopaminergic neurons project to the striatum, which is a proper dopaminergic target area. Also in mutants in which the MHO is shifted rostrally, dopaminergic and serotonergic neurons are relocated at the newly positioned MHO. However, in these mice the size ratio between these two cell populations is changed in favour of the serotonergic cell population. To investigate whether the position of the MHO during embryogenesis is also of functional relevance for adult behaviour we tested mice with a caudally shifted MHO and report that these mutants show a higher locomotor activity. Taken together, we provide evidence that the position of the MHO determines the location and size of midbrain dopaminergic and hindbrain serotonergic cell populations in vivo. In addition our data suggest that the position of the MHO during embryogenesis can modulate adult locomotor activity.

Keywords: mid-hindbrain organizer, dopamine, serotonin, attention-deficit hyperactivity disorder

Olfactory-learning induced reduction in post-burst AHP in piriform cortex neurons is due to a decrease in $\mathbf{S I}_{\mathrm{AHP}}$ Brosh I. ${ }^{1}$ and Barkai E.

Zlotowski Center for Neuroscience, Ben-Gurion University Beer Sheva; ${ }^{2}$ Center for Brain and Behavior, Haifa University We have previously shown that olfactory learning results with reduced post-burst afterhyperpolarization (AHP) in layer II pyramidal neurons of the piriform cortex. In the present study we examine which of the currents that generate the post-burst AHP ( $\mathrm{sI}_{\mathrm{AHP}}$ and $\mathrm{I}_{\mathrm{AHP}}$ ) is reduced after learning.

Rats were trained in an olfactory discrimination task to distinguish between positive and negative odor cues until they demonstrated rule learning.

Three days after completion of training, intracellular recordings from pyramidal neurons were performed in piriform cortex brain slices. As previously shown, AHP amplitude was 
significantly smaller $(P<0.02$, one way ANOVA) in neurons from trained rats, compared with neurons from pseudo trained and naive rats $(-4.98 \pm 0.30 \mathrm{mV}, \mathrm{n}=32$ in trained, $-6.79+0.38$ $\mathrm{mV}, \mathrm{n}=23$ in pseudo trained and $-6.76 \pm 0.37 \mathrm{mV}, \mathrm{n}=\mathbf{2} 4$ in naive). In the presence of $10 \mu \mathrm{M}$ NE, AHP amplitude in neurons from trained rats did not differ from those of pseudo trained or naive rats $(-6.32 \pm 0.71 \mathrm{mV}, \mathrm{n}=14$ in trained, -5.20 $\pm 0.54 \mathrm{mV}, \mathrm{n}=12$ in pseudo trained and $-5.64 \pm 0.40 \mathrm{mV}, \mathrm{n}=24$ in naive). In the presence of $50 \mathrm{nM}$ of the specific $\mathrm{I}_{\mathrm{AHP}}$ blocker Apamin, AHP amplitude remained significantly smaller $(\mathrm{P}<0.03$, one way ANOVA) in neurons from trained rats, compared with controls $(-2.76 \pm 0.41 \mathrm{mV}, \mathrm{n}=17$ in trained, $-4.67 \pm 0.59 \mathrm{mV}, \mathrm{n}=16$ in pseudo trained and $-4.73 \pm 0.31 \mathrm{mV}$, $\mathrm{n}=9$ in naive). Furthermore, the reduction in AHP amplitude was stronger in neurons from trained rats $(45 \%)$ then that in pseudo trained $(31 \%)$ or naive rats $(30 \%)$.

These data indicate that learning induced post-burst AHP reduction is accompanied by reduction in ratio of $\mathrm{sI}_{\mathrm{AHP}} / \mathrm{I}_{\mathrm{AHP}}$ conducnatnes. We suggest that the differential effect of noradrenaline is due to this change.

Keywords: olfactory-learning, piriform cortex, AHP, noreadrenaline

Identification of a new gene for otosclerosis in Israeli family Brownstein Z. ${ }^{1}$, Frydman M., ${ }^{1,2}$ and Avraham K.B. ${ }^{1}$ Dept. of Human Genetics and Molecular Medicine, Sackler School of Medicine. Tel Aviv University, Tel-Aviv 69978, ${ }^{2}$ Genetics Institute, Haim Sheba Medical Center, Tel Hashomer Otosclerosis is a common bone disorder with a prevalence of $0.2 \%-1 \%$ among adults. It is characterized by isolated endochondral bone sclerosis of the labyrinthal capsule. Otosclerosis leads to a progressive hearing impairment which begins as a conductive hearing loss (HL), due to a fixation of the stapedial footplate in the oval window, and might develop into a mixed to sensorineural (SN) HL, caused by cochlear otosclerotic foci. The age of onset is usually $20-40$ years and in most cases both ears are involved. The mode of inheritance is autosomal dominant, with reduced penetrance. Thus far, the chromosomal locations of three loci for otosclerosis have been reported on chromosomes 15q25-q26, 7q34-36 and 6p21.3-22.3

A family with otosclerosis (Family $\mathrm{O}$ ) is participating in our research project. Twenty one members of Family $\mathrm{O}$ have been investigated. Nine have progressive HL, with large variability, and onset in the early thirties. Two individuals that were operated on were confirmed as having otosclerosis. We excluded linkage to the three loci reported, leading us to perform a whole genome scan at the Laboratory of DNA Analysis at the Institute of Life Sciences, Hebrew University of Jerusalem, in order to identify the chromosomal location of the causative gene. Four hundred polymorphic markers were evaluated. We have identified three possible regions of linkage, on chromosomes 2,16 , and 20 . We are now genotyping with additional microsatellite markers in these three regions to determine which region is indeed linked to hearing loss in Family $\mathrm{O}$.

Keywords: otosclerosis, conductive hearing loss, linkage, genome scan

\section{Cortisol levels in traumatized patients presenting to the ER: A preliminary report \\ Brunet A., Birmes P. and Meaney M. \\ Douglas Hospital and McGill University.}

Low cortisol among individuals with Posttraumatic Stress Disorder (PTSD) has been taken as evidence that PTSD is associated with enhanced negative feedback of the hypothalamic-pituitary-adrenal (HPA) axis. It remains unclear however whether this pattern reflects (a) a risk factor for PTSD, (b) a universal consequence of trauma exposure, or (c) a long-term consequence of having PTSD. In order to examine this issue, we followed for 6 months 31 individuals presenting to the emergency department (ED) as a result of trauma exposure. Salivary cortisol was collected 5 days post-trauma as well as $1,2,4$, and 6 months post trauma. Six months post-trauma 9 individuals had PTSD while 22 did not. The analyses revealed a main effect for diagnosis, $F(1,29)=20.46$, $\mathrm{p}<.001$, with the PTSD group having lower salivary cortiso levels from the onset. There was a marginally significant effect for time, $F(4,26)=2.35, p p<.06$, suggesting that cortisol levels increase with the passage of time and as symptoms of PTSD decrease. No time by group interaction was observed. Those preliminary results suggest that low cortisol may be both a risk factor and a consequence of trauma exposure.
T cell-mediated protective autoimmunity after spinal cord injury affects immune-related activity of glial cells in vitro and in vivo

Butovsky O. and Schwartz M.

Dept. of Neurobiology, Weizmann Institute of Science, Rehovot 76100

Studies in our laboratory have demonstrated that passive or active vaccination with $T$ cells specific to myelin self-antigens such as myelin basic proteins (MBP) can reduce the spread of damage after central nervous system (CNS) injury. This phenomenon was defined by our group as "protective autoimmunity" (Moalem et al. Nat Med 5:49-55 [1999]; Hauben et al., J Neurosci 20, 6421-6430 [20001). Our in vivo studies suggest that $T$ cells affect the behavior of microglia/macrophages. When spinal cord injury in rats was followed by active or passive T-cell vaccination, accumulation of $\mathrm{T}$ cells in the vicinity of the lesion site was accompanied by a dramatic increase in MHC-II expression on macrophages/microglia, with a concomitant increase in expression of the costimulatory molecule B7-2, possibly augmenting the ability of the microglia to support antigen-dependent $T$ cell activation (Butovsky et al., FASEB J $15,1065[2001])$

Our in vitro studies showed that co-culturing of microglia with autoimmune $\mathrm{T}$ cells resulted in up-regulation of the expression of MHC-II and B7-2 (both reminiscent of the activity of antigen-presenting cells) as well as increased expression of TNF-(, TNF-RI and TNF-RII in early interaction and later on, decreased expression of TNF-RII (possibly associated with termination of the microglial activity). We suggest that autoimmune $\mathrm{T}$ cells exert their protective effect through dialog with local microglia and invading macrophages, enabling these cells to express their beneficial activity, while at the same time imposing strict regulation so as to avoid their potentially detrimental long term effects.

Keywords: CNS ,spinal cord injury, secondary degeneration, autoimmune neuroprotection, microglia/astrocytes, TNF/TNF-RI/RII

\section{The ischemic synapse and the role of mitochondria \\ Carlen P.}

University of Toronto, Canada

Mitochondria are increasingly implicated in neurodegenerative mechanisms from several perspectives, including intracellular calcium regulation, free radical production, energy utilization, and release of apoptotic factors. Synaptic transmission could be an early and sensitive target for cerebral ischemia and other neurodegenerative processes.

We have shown that an eight min ischemic (hypoxia/hypoglycemia) insult to an organotypic hippocampal slice culture, promoted glutamate-mediated generation of free radicals with concomitant elevation of intracellular calcium (Perez Velazquez et al., 1997). In this same model, the mitochondrial complex I inhibitor, rotenone, the mitochondrial permeability transition blocker, cyclosporin $\mathrm{A}(\mathrm{Cs} \mathrm{A})$, and a blocker of NAD + , nicotinamide, decreased ischemia-induced free radical generation and increased mitochondrial calcium (Frantseva et al., 2001). Interestingly, CsA did not diminish the increase in the cytoplasmic calcium, but did reduce the increased mitochondrial calcium, suggesting that mitochondrial calcium could be the most important mediator of neurodegenerative processes. Preliminary experiments, in acutely prepared rat hippocampal slices, have shown that during and following $8 \mathrm{~min}$. of hypoxia/hypoglycemia, stratum radiatum evoked synaptic transmission in the CAl subfield is depressed along with an increase in presynaptic cytoplasmic and mitochondrial calcium, which persists for up to one hour following the ischaemic insult. The presynaptic increase in calcium is reduced by BAPTA-AM. We hypothesize that the neuroprotective actions of intracellular calcium chelation could be by limiting rises in intramitochondrial calcium and that the maintenance of 'healthy' mitochondria will be neuroprotective against ischaemic insults.

Supported by the CIHR

Keywords: ischemia, synaptic transmission, mitochondria, presynaptic calcium 
Functional $\mathrm{GABA}_{\mathrm{B}}$ receptors are lost at the hippocampal mossy fibre synapse after status epilepticus Chandler KE. ${ }^{1}$, Princivalle AP. ${ }^{2}$, Bowery NG. ${ }^{2}$, Walker MC. ${ }^{1}$ and Kullmann DM.

${ }^{I}$ Dept. of Clinical and Experimental Epilepsy, Institute of Neurology, UCL, London, UK, ${ }^{2}$ University of Birmingham, UK Activity dependent GABA release reduces mossy fibre (MF) field excitatory post-synaptic potentials (fEPSPs) in hippocampal slices (Min et al, Neuron 1998; 21:561-70, Vogt \& Nicoll, PNAS 1999;96:1118-22). We have shown that this form of $\mathrm{GABA}_{\mathrm{B}}$ receptor mediated heterosynaptic depression (HD) is lost after SE (Chandler et al J Physiol 2001.536P). Is this due to reduced presynaptic $\mathrm{GABA}_{\mathrm{B}}$ receptors on MFs? We recorded extracellular fEPSPs in stratum lucidum (where MFs synapse on $C A 3$ pyramidal neurons) in acute hippocampal slices from control rats and rats after pilocarpine-induced SE. We applied the $\mathrm{GABA}_{B}$ receptor agonist baclofen at increasing concentrations. The fEPSP was less sensitive to baclofen after $S E$, relative to control slices $(p<0.05$, unpaired $t$ test $)$. $G A B A_{B}$ receptor mediated HD in post-SE slices could not be significantly increased by blocking GABA uptake with N0711 $(20 \mu \mathrm{M})(\mathrm{n}=4)$. These data suggest that reduced $\mathrm{GABA}_{B}$ receptor mediated HD is due to loss of functional receptors and not to enhanced uptake. We then asked if there was a change in receptor number after $\mathrm{SE}$ by detecting binding of the $\mathrm{GABA}_{B}$ receptor antagonist $\left.{ }^{3} \mathrm{H}\right] \mathrm{CGP} 62349$ autoradiographically. Maximal binding $\left(\mathrm{B}_{\max }\right)$ of $\mathrm{GABA}_{\mathrm{B}}$ receptors by $\left[{ }^{3} \mathrm{H}\right] \mathrm{CGP} 62349$ in stratum lucidum was significantly decreased after SE $\left[\mathrm{B}_{\max }(\mathrm{fmol} / \mathrm{mg}\right.$ tissue) for controls $1322+1-68, n=5 ; B_{\max }$ (fmol/mg tissue) 24 hours after SE $1080+1-56, n=4 ; p=0.033$ for difference]

We have provided evidence that loss of $\mathrm{GABA}_{\mathrm{B}}$ receptor-mediated heterosynaptic depression after $S E$ is due to reduced $\mathrm{GABA}_{\mathrm{B}}$ receptors on mossy fibre terminals. This may increase excitability of the hippocampus after SE and play a role in epileptogenesis

Keywords: epilepsy, $\mathrm{GABA}_{B}$ receptor, mossy fibre

The neuropeptides GnRH-II and GnRH-I interact directly with normal and cancer human $T$-cells and trigger de novo gene expression of a unique laminin receptor, adhesion, chemotaxis and homing in vivo to specific organs Chen A. ${ }^{1}$, Ganor Y. ${ }^{1}$, Rahimipour S. ${ }^{.}$, Ben-Aroya N. ${ }^{1}$, Koch Y. ${ }^{1}$ and Levite M.1,2

${ }^{I}$ Dept. of Neurobiology, Weizmann Institute of Science. Rehovot 76100; ${ }^{2}$ The Sackler Faculty of Medicine, Tel-Aviv University, Tel-Aviv 69978

Can T-cells be driven to de novo gene-expression under the exclusive command of a neuropeptide?

In this study, we investigated whether T-cells can be directly activated by gonadotropin-releasing-hormone-II (GnRH-II), a unique neuropeptide conserved through 500 million years of evolution, and recently identified in the brain of various mammals, as well as in various peripheral tissues. We found that exposure of normal human or mouse $\mathrm{T}$-cells to either GnRH-II or GnRH-I (the two neuropeptides sharing 70\% homology but encoded by different genes) leads to de novo gene-transcription of various genes, among them the cell-surface-expressed $67-\mathrm{kDa}$ non-integrin laminin-receptor, that plays a key role in cellular adhesion and migration, tumor invasion/metastasis, and specific infections.

GnRH-II or GnRH-I also directly induced cell-surface-expression of the 67-kDa-laminin-receptor, adhesion to laminin, chemotactic migration towards SDF-1 $\alpha$, and augmented entry in vivo of metastatic T-lymphoma into specific organs. Further, homing of normal T-cells occurred to a lesser extent in GnRH-I-knockout mice than in normal syngeneic mice. A specific GnRH-I receptor-antagonist blocked the induced effects of GnRH-I but not of GnRH-II suggesting signaling through distinct putative binding sites Finally, we found that normal human and leukemia T-cells produce GnRH-II and GnRH-l.

Taken together, we suggest that GnRH-II and GnRH-I, secreted from nerves or autocrine/paracrine sources, interact directly with T-cells, and trigger specific gene-transcription adhesion, migration and homing of $T$ cells across blood and tissue barriers. We further speculate that these GnRH-T-cell interactions may be beneficial in conditions of normal T-cell behavior, but detrimental when cancer or autoimmune T-cells are involved.

Keywords: GnRH, T-cells, laminin-receptor, neuropeptides, neuroimmunomodulation
$\mathrm{P}_{2} \mathrm{Y}_{4}$ receptors in satellite glial cells in trigeminal ganglia of mice

Cherkas P.S. ${ }^{1}$. Weick M. ${ }^{2}$, Hartig W. ${ }^{2}$, Pannicke T. ${ }^{2}$, Huang T.Y. ${ }^{1}$, Tal M. ${ }^{\mathrm{f}}$, Reichenbach A. ${ }^{2}$ and Hanani M.

${ }^{1}$ The Hebrew University-Hadassah Medical School. Jerusalem: ${ }^{2}$ Paul Flechsig Institute for Brain Research, University of Leipzig, Germany

Purinergic receptors have been found in sensory neurons, but there is no information on their presence in satellite glial cells (SCs) of sensory ganglia. SCs closely envelop each neuron and thus can respond quickly to substances released from neurons. We studied the actions of ATP on SCs in isolated mouse trigeminal ganglia. Incubation of the ganglia in medium containing $\mathrm{Ca}$-sensitive dye fura-2 AM led to its preferential uptake by the SCs. Putative neurotransmitters were tested for their ability to increase the intracellular $\mathrm{Ca}^{2+}$ concentration $\left[\mathrm{Ca}^{2+}\right]_{\text {in. }}$. Fluorescence above $510 \mathrm{~nm}$ was excited at $340 \mathrm{~nm}$ $\left(\mathrm{F}_{340}\right)$ and $380 \mathrm{~nm}\left(\mathrm{~F}_{380}\right)$, and images were recorded every 3 seconds. The ratio $F_{340} / F_{380}$ was used to describe relative changes in $\left[\mathrm{Ca}^{2+}\right]_{\text {id. }}$. Application of substance $\mathrm{P}$ and glutamate $\left(100 \mu \mathrm{M}\right.$ each) did not cause any change in $\left[\mathrm{Ca}^{2+}\right]_{\text {in }}(22$ and 20 cells). Application of $100 \mu \mathrm{M}$ of ATP caused an increase in $\left[\mathrm{Ca}^{2+}\right]_{\text {in }}$ in the SCs $(n=44)$. In the next experiments we attempted to determine which receptor was responsible for this response. In $\mathrm{Ca}^{2+}$-free extracellular solution an increase in $\left[\mathrm{Ca}^{2+}\right]_{\text {in }}$ was recordable, suggesting the involvement of $\mathrm{P} 2 \mathrm{Y}$ receptors. Moreover, the same effects were recorded when UTP (a P2Y agonist, $100 \mu \mathrm{M}$ ) was used $(\mathrm{n}=19)$. Application of adenosine $(100 \mu \mathrm{M})$ did not have an effect $(n=24)$, indicating that the ATP response was not due to breakdown of ATP and also not mediated by adenosine receptor. These results indicate that $\mathrm{P} 2 \mathrm{Y}_{2}$ and/or $\mathrm{P} 2 \mathrm{Y}_{4}$ receptors mediate the $\mathrm{Ca}^{2+}$ response to ATP. To identify which receptor subtype was involved we immunostained the trigeminal ganglia for $\mathrm{P}_{2} \mathrm{Y}_{1}, \mathrm{P} 2 \mathrm{Y}_{2}$ and $\mathrm{P}_{2} \mathrm{Y}_{4}$ receptors. We found immunoreactivity only for $\mathrm{P}_{2} \mathrm{Y}_{4}$ in SCs. We conclude that SCs express a G-protein-linked purinergic receptor $\mathrm{P} 2 \mathrm{Y}_{4}$, whose activation leads to $\left[\mathrm{Ca}^{2+}\right]_{\text {in }}$ elevation. These findings suggest a possible mode of communication between neurons and satellite glial cells in trigeminal ganglia.

Keywords: trigeminal ganglion, satellite glial cells, $\mathrm{P}_{2} \mathrm{Y}_{+}$ receptor

Knockout of gap junctions in the inferior olive "knocks-in" compensatory mechanisms that allows for the production of oscillations in single olivary neurons

Chorev E. ${ }^{1}$, Devor A. ${ }^{\text {, }}$, Manor $Y^{2}{ }^{2}$, de Zeeuw C.I. ${ }^{3}$ and Yarom Y. ${ }^{\top}$ Dept. of Brain Research, Life Sciences Institute. Hebrew University of Jerusalem. Jerusalem: ${ }^{2}$ Dept. of Life Sciences and Zlotowski Center for Neuroscience. Ben-Gurion University of the Negev, Beer-Sheva: ${ }^{3}$ Dept. of Neuroscience. Erasmus University Rotterdam, Rotterdam. The Netherlands

Olivary neurons exhibit subthreshold oscillations (STOs) of their membrane potential. These oscillations depend on electrical coupling as well as on intrinsic properties. To study the exact role of STOs in behavior we used CX36 knockout (KO) mice. EM cross-section reveals that the KO mice have abnormal gap junctions-like structures, which appear dysfunctional. This is further supported by the lack of dve coupling and the fact that direct current transfer could not be measured between $\mathrm{KO}$ olivary neurons

The loss of functional gap-junctions did not impair the ability of these neurons to generate calcium-spikes in response to depolarizing currents steps given from a depolarized or hyperpolarized baseline. However the $\mathrm{KO}$ neurons have developed a unique property that enables them to produce calcium spikes in response to hyperpolarizing current steps. The oscillatory nature of these cells also differed. In $50 \%$ of the wild type (WT) cases, olivary neurons exhibited yoltage independent subthreshold osciflations. In contrast, in $\mathrm{KO}$ neurons, oscillations appeared when the membrane potential was hyperpolarized in a narrow range of values.

Using the dynamic clamp technique we demonstrated that WT neurons could be transformed into $\mathrm{KO}$-like neurons by decreasing their leak conductance, increasing their low threshold calcium (L.TC) conductance or changing the LTC kinetics. We conclude that the absence of electrical coupling triggers compensatory processes that lead to rhythmic activity in individual neurons.

We hypothesize that STOs in olivary neurons play a pivotal role in cerebellar function. Under normal conditions, STOs are generated and modulated by the electrical coupling. In the absence of electrical coupling, the system is modified such that each neuron becomes a conditional oscillator. The modulation 
is thus achieved by an hyperexcitable response to chemical synapses. With such compensatory mechanisms, it is not surprising to find only minute differences in the behaviors of $\mathrm{KO}$ and WT

Keywords: inferior-olive, gap junctions, oscillation, knockout

Recording action potentials by depletion type floating gate P-channel MOS transistor

Cohen A. ${ }^{1}$, Yitshaik S. ${ }^{1}$, Shappir Y. ${ }^{1}$, Shwartzglass O. ${ }^{1}$, Borghs $\mathrm{G}^{2}$ and Spira M.E.

${ }^{I}$ Faculty of Science, The Hebrew University of Jerusalem, Jerusalem; '2 Imec Leuven, Belgium

We report the realization of electrical coupling between neurons and depletion type floating gate (FG) p-channel MOS transistors. The devices were realized in a shortened $0.5 \mu \mathrm{m}$ CMOS technology. Increased boron implant dose was used to form the depletion type devices. Post CMOS processing steps were added to expose the devices sensing area

The neurons are coupled to the polycrystalline Silicon (PS) FG through $420 \mathrm{~A}$ thermal oxide in an area which is located over the thick field oxide away from the transistor. The combination of coupling area pad having a diameter of $10-15 \mu \mathrm{m}$ and sensing transistor with $\mathrm{W} / \mathrm{L}$ of $50 / 0.5 \mu \mathrm{m}$ results in capacitive coupling ratio of the neuron signal of about 0.5 together with relatively large transistor trans-conductance.

The combination of the FG structure with depletion type device, leads to the following advantages: a) no DC bias between the biological solution and the transistor with direct consequences to the neuron as well as the silicon die durability. b) The sensing area of the neuron activity is separated from the active area of the transistor. Thus, it is possible to design the sensing area and the channel area separately. c) The channel area, which is the most sensitive part of the transistor, can be insulated and shielded from the ionic solution. d) The option to add a switching transistor to the FG and use the FG also for the neuron stimulation.

Keywords: MOS transistor, action potential

The neural activity in rat motor cortex changes during the learning of a conditional sensory discrimination task Cohen D. and Nicolelis M.A.L.

Dept. of Neurobiology, Duke University Medical Ctr., Durham, NC 27705

Neural activity in motor cortex has been intensively studied for many years. Yet, little is known about the changes that occur in these neurons' activity as animals learn a conditional auditory discrimination task. In this study, arrays of microwires were chronically implanted in motor cortex (MI) of adult rats to allow the recording of single unit activity as the rats learn to associate an arbitrary tone with a movement. This approach allowed us to characterize changes in cortical activity due to learning at the level of single neurons as well as the ensemble. Adult rats were trained to nose poke a center hole and wait for a go cue which was either a high or a low tone. Depending on the tone, the rat was required to nose poke a hole located either to the right or to the left of the center hole. Following correct trials, the rat received water reward. In this task, the rats learn to associate each tone with a specific movement in addition to improving the movement performance via repetitions.

Our results show that during the learning process, the behavioral attributes of reaction time, movement time and error frequency decreased with practice. The improvement in task performance was accompanied by changes in the firing patterns of neurons associated with the task. These changes included sharpening of the response to the task related events and modification of the average firing rate

Keywords: chronic recording, motor learning

ERK activation is correlated with synaptic modifications after odor rule learning in the piriform cortex

Cohen $\mathrm{S}^{1}$, Shiboleth A. ${ }^{2}$, Rosenblum $\mathrm{K}^{2}{ }^{2}$ and Barkai E. ${ }^{2}$ ZZlotowski Center for Neuroscience, Ben-Gurion University, Beer Sheva; ${ }^{2}$ Center for Brain and Behavior, Haifa University, Haifa

We have shown previously that post-burst afterhyperpolarization (AHP) and paired pulse facilitation $(\mathrm{PPF})$ is reduced in piriform cortex of pyramidal neurons, days after olfactory-trainning. We assume that this long term cellular modifications subserve at least part of the odor rule learning and are looking into possible molecular mechanisms for these long term modifications (See also Shiboleth et al.) Extra cellular regulated kinas (ERKL/II) were found by others and us to play major role in learning and memory as well as synaptic plasticity in cortical areas. We began to study the possible involvement of ERKI/II in long-term modulation of intrinsic and synaptic properties correlated with odor rule learning in the piriform cortex. The specific MEK inhibitor PD98059 $(40 \mu \mathrm{M})$ caused a significant increase in PPF amplitude in neurons from trained rats only. Consequently, the difference in PPF amplitude between trained and pseudo rats was diminished (from $1.22+0.25, \mathrm{n}=21$ to $1.56+0.29, \mathrm{n}=7$ in trained and from $1.34+0.20, n=6$ to $1.44+\overline{0} .18, n=5$ in pseudotrained). PD98059 did not have an effect on post-burst AHP amplitude in any group on neurons (from $6.25 \pm 2.29$, $\mathrm{n}=44$ to $6.53+2.72, \mathrm{n}=19$ in trained and from $7.82+1.74, \mathrm{n}=20$ to $7.31+1.7 \overline{4}, \mathrm{n}=8$ in pseudotrained). Using sub-cellular fractionation and quantitative immunoblot analysis of anti phospho-specific antibody and protein antibodies we are examining ERKI/II activation in the piriform cortex. In preliminary research we detected $20 \%$ increase $(n=4)$ in ERKI/II activation in the cytosolic fraction of trained $(n=4)$ compare to control rats. We conclude that ERKL/II is involved with long-term modulation of synaptic transmission in the piriform cortex of trained rats but not in post burst AHP 3 days following training.

Keywords: olfactory-learning, piriform cortex, paired-pulse facilitation, ERK

Prosapsin induces dendritic clustering of GluR1 and spine

formation ${ }_{\text {Cove }{ }^{1,2}, \text { Morales C. }}^{3}$ and Baranes D.,2

Dept. of Life Sciences, Ben-Gurion University of the Negev.

Beer-Sheva, ${ }^{2}$ Zlowtovski Center for Neuroscience, Beer Sheva: ${ }^{3}$ Dept. of Anatomy and Cell Biology, McGill University.

Montreal, Canada

Formation of post-synapic spines and mobilization of glutamate receptors into spines are part of the mechanism underlying long-term synaptic plasticity (Muller et al., Hippocampus 10(5):596-604 [2000]). However, little is known of the factors inducing these processes. Prosaposin, a precursor of the lysosomal saposins, has a $71 \mathrm{kD}$-secreted variant with neurotrophic activity, which can reduce synaptic degradation in hippocampus during ischemia (Sano et al., $B B R C$ 204(2):994-1000 [1994]). Nonetheless, it is unclear whether it directly influences synaptic development and function.

We found that in hippocampal neurons prosaposin is localized in vesicles within dendrites, but is absent from axons and synaptic connections. Depletion of prosaposin in knockout mice is associated with an approximately 3-fold lower expression level of dendritic Glutamate Receptor 1 (GluRI) in the hippocampal dentate gyrus and CA! regions compared to the wild type. Supporting this, application of an anti-prosaposin antibody to the growth medium of hippocampal cultures reduces dendritic GluRI cluster density by $44 \%$ and GluR cluster size by $56 \%$. These effects are specifically on GluRl since the treatment with anti-prosaposin has no effect on NMDAR1 clustering.

Furthermore, anti-prosaposin reduces the density of GluR1-containing spines by $88 \%$, whereas application of exogenous prosaposin increased this density by $91 \%$

Our results show that extra-cellular activity of prospaosin clusters GluRI, but not NMDARI in dendrites, and promotes spine formation.

Keywords: dendritic spines, glutamate receptor, prosaposin

The impact of intracellular A-beta peptides

Cuello A.C., Echeverria V., Dowd E., Lopez E., Vercauteren

F., Bell K., Ribeiro-da-Silva A. and Szyf M.

McGill University, Department of Pharmacology and

Therapeutics, Canada

Extracellular A- $\beta$ peptides unquestionably displav toxic effects contributing to the Alzheimer's Disease (AD) pathology: However, intracellular accumulation of $A \beta$ also occurs at early stages of $A D$. In vitro studies have shown that intracellular $A \beta$. (A $\mathrm{Bi}$ ) can disrupt cellular organelles and MAP kinases. We have continued investigations regarding the consequences of elevated levels of $\mathrm{ABi}$ in a rat transgenic model, expressing human transgene genes related to familial forms of $A D$, whose CNS pathology is limited to intraneuronal accumulation of $A \beta$ in the hippocampus and neocortex. In this transgenic rat mode we have observed that the presence of intraneuronal $A \beta$, in the absence of amyloid plaques, is sufficient to provoke up-regulation of the MAP kinase ERK2 in the hippocampus at 9 months. This is a dysregulation, which appears specific, since no changes were observed in other putative tau kinases. such as GSK $3 \quad \alpha-\beta$ and CDK5. These rats displayed a concomitant increase in the level of tau phosphorylation at ERK2 target sites. Confocal microscopy studies revealed that neurons displaving $A \beta i$, also manifest changes in the Golgi complex and lysosomes. Interestingly, these morphological and biochemical alterations were followed by behavioral cognitive impairments which became apparent at 16 months of 
age, thus suggesting that in the absence of amyloid plaques, accumulation of intracellular $A \beta$ peptides is sufficient to initiate steps in the tau-phosphorylation cascade as well as to provoke late impairments of higher CNS functions. We are currently investigating other potential protein dysregulations within our transgenic rat model of early $A D$ pathology, in an effort to identify the molecular pathology preceding plaque formation

Supported by grant number MOP-37996 from the Canadian Institutes for Health Research.

Keywords: intracellular A-beta; transgenic rat; MAP kinases; tau-phosphorylation

Effects of corticosterone on dendritic spine density in cultured rat hippocampal neurons

Dachir S. ${ }^{1}$, Greenberger V. ${ }^{2}$ and Segal M. ${ }^{2}$

${ }^{T}$ Dept. of Pharmacology, Israel institute for Biological

Research, Ness Ziona 74100; ${ }^{2}$ Dept. of Neurobiology,

Weizmann Institute of Science, Rehovot 76100

Dendritic spines, the site of synaptic connections between central neurons, play an important role in information processing and memory formation. Corticosterone, one of the major stress-induced hormones in mammals, may have profound effects on the morphology and functions of specific brain areas. One of the brain areas most affected by stress hormones is the hippocampus, a limbic structure, known for its involvement in learning and short-term memory. To examine the effects of corticosterone on the structure and functions of hippocampal neurons in relative isolation we exposed dissociated hippocampal neurons cultured for two weeks in-vitro to $0.1 \mu \mathrm{M}$ corticosterone for $24 \mathrm{hrs}$, and measured the effect of the hormone on several morphological indices. The most striking and consistent effect of corticosterone was a decrease of about $26 \%$ in the density of dendritic spines without a concomitant change in other morphological parameters, including soma size, and number of primary dendrites per neuron. Also, there was no difference in the size of the spines as a result of exposure to corticosterone. Interestingly, the presence of the steroid antagonist RU486 by itself caused a significant increase in spine density above control level, and blocked the effects of corticosterone.

Acute $(30 \mathrm{~min})$ corticosterone treatment caused a significant increase in NMDA receptor-independent rise in [Ca]i reactivity of the cultured neurons to topical application of glutamate. These results indicate that corticosterone causes pruning of dendritic spines by increasing calcium load through an enhanced voltage gated calcium channel activity, as suggested elsewhere

Keywords: corticosterone, hippocampus, dendritic spines

Intermittent pentobarbital microinfusion into the rat MPTA induces long lasting anesthesia

Del Canho $\mathrm{S}$. and Devor $\mathrm{M}$

Dept. of Cell and Animal Biology. Institute of Life Sciences,

Hebrew University of Jerusalem. Jerusalem

Aims of Investigation: We have identified an area in the rat brainstem mesopontine tegmentum (MPTA) where minute pentobarbital microinjections induce flaccidity, analgesia, slow-wave EEG and apparently loss of consciousness (Devor \& Zalkind Pain, 2001). Following single pentobarbital bolus microinjection the anesthetic state typically lasts 20-30 min. In many types of neurobiological research it would be advantageous to anesthetize animals without flooding the neural tissue under investigation with anesthetic agents. We therefore asked whether more prolonged anesthesia can be generated by pentobarbital microinfusion into MPTA

Methods: Wistar-derived Sabra strain rats $(280-300 \mathrm{~g})$ were anesthetized and implanted with bilateral stainless steel guide-cannulae aimed at MPTA. After full recovery (one week) a $30 \mathrm{~g}$ microinjection needle was inserted through each guide cannula bringing its tip within MPTA. We selected rats in which pentobarbital micronjection induced moderate to deep anesthesia (11-16 on the previously used 16-point scale)

Results: After inducing anesthesia with a bolus microinjection ( $100 \mu \mathrm{g}$ in $0.5 \mu \mathrm{l}$ per side), microinfusion was initiated $(10 \mu \mathrm{g}$ in $0.05 \mu \mathrm{J}$ per minute). Animals tended to lighten, awakening after $40 \mathrm{~min}$. However, intermittent microinfusions of $20 \mu \mathrm{g}$ in $0.1 \mu \mathrm{l}$ per minute or $40 \mu \mathrm{g}$ in $0.2 \mu \mathrm{l}$ per minute for periods of $5-10 \mathrm{~min}$ every $30 \mathrm{~min}$ induced stable anesthesia for periods of 2-3 hours (6 experiments in 4 rats).

Conclusion: Prolonged anesthesia can be obtained with repeated brief microinfusions, although similar doses infused continuously at lower rate, appear to be less effective. MPTA microinfusion is a potentially useful tool for inducing stable, prolonged anesthesia without delivering drugs to the systemic circulation.

Keywords: microinjection, general anesthesia, microinfusion
Differential pattern of CREB activation in the rat brain after conditioned aversion as a function of the cognitive process engaged: taste vs. context association Desmedt A., Hazvi S. and Dudai Y.

Dept. of Neurobiology, Weizmann Institute of Science, Rehovot 76100

Ample data indicate that cAMP-response element-binding protein (CREB) is essential for the formation of long-term memory in various species and learning systems (Silva et al, Ann Rev Neurosci 21:127-148 [1998]). This implies that activated CREB could be used as a metabolic marker to delineate neuronal circuits that subserve specific items in memory. However, the question how CREB activation in identified neurons contributes to the specificity of the internal representation encoded by the relevant circuit is still unexplored. As a first step in this direction, we describe here the differential activation of CREB in the rat brain as a consequence of two related yet distinct forms of aversive conditioning, conditioned taste aversion (CTA) and conditioned context aversion. Using immunohistochemistry, we found that CTA induces strong CREB activation in the insular cortex (IC) and the lateral septum (LS), but not in the parietal cortex (PC) and the medial septum (MS). In contrast contextual conditioning resulted in strong activation in the PC and MS, but not in the IC and LS. These findings are congruent with a model that links a differential pattern of activity within the LS and the MS with the acquisition of elemental vs. contextual conditioning (Desmedt et al, Eur $J$ Neurosci 11:3913-3921 [1999]). The findings also support the hypothesis that CREB activation may specify learning-dependent circuits as a function of the type of cognitive process engaged

Keywords: conditioned aversion, septum, insular cortex, parietal cortex

\section{Induction of brain amyloid beta deposits by inhibition of Neprilysin \\ Dolev I. and Michaelson D.M}

Faculty of Life Science, Tel Aviv University, Tel Aviv 69978

Brain amyloid beta $(\mathrm{A} \beta)$ deposits are a major histopathological hallmark of Alzheimer disease (AD) and are believed to play an important role in the pathology of the disease. The mechanisms underlying the aggregation and deposition of $A \beta$ are not yet known. Recent studies revealed that $A \beta$ deposits occur in mice transgenic to amyloid precursor protein (APP) The occurrence of these deposits is age dependent and it is not yet known whether they can be cleared or degraded in vivo. The brain neutral endopeptidase, Neprilysin, is a major $A \beta$ degrading protease. In the present study we inhibited brain neprilysin activity by prolonged i.c.v injection of Thiorphan, a selective inhibitor of Neprilysin, and examined the resulting effects on the rate and extent of $A \beta$ deposition in the brain as well as on the extent to which these deposits can be cleared and degraded. Accordingly, i.c.v administration into the lateral ventricle of Thiorphan by Alzet osmotic pumps $(0.5 \mathrm{mM}$ at a flow rate of $0.25 \mu \mathrm{l} /$ hour) revealed a time dependent increase in the number of immunohistochemicaly detectable $A \beta$ deposits The level of $A \beta$ deposits increased as a function of time for at least 1 month after initiation of treatment. Examination of the spatial distribution of the $A \beta$ deposits in the brain revealed maximal levels in the cortical areas whose ventral surface was the largest. The extent to which $A \beta$ deposition is affected by AD genetic risk factors such as the apoE genotypes and the degree to which these deposits can be cleared from the brain will be discussed

Keywords: $A \beta$, neprilysin, Alzet pumps, apoE

Protein kinase $\mathrm{C} \varepsilon$ is involved in the adenosine-activated mechanism of protection against ischemia/reperfusion in primary neuronal cultures

Di Capua N., Sperling O. and Zoref-Shani E

Dept. of Clinical Biochemistry, Sackler Faculty of Medicine Tel-Aviv University, Tel-Aviv 69978

We have demonstrated before in primary rat neuronal cultures (Reshef et al., Pharmac. \& Ther., 87:15]-159 [2000]), that adenosine activates a signal transduction pathway ("the adenosine mechanism"), conferring resistance against ischemia/reperfusion insult. In continuation to our previous studies, we now provide proof for the participation of $\mathrm{PKC} \varepsilon$ in the neuronal adenosine-induced protective signal transduction pathway. Our experimental model was that of primary rat neuronal cultures exposed to chemical ischemia (iodoacetate), followed by reperfusion. The protection signal was initiated by exposure to R-PIA, a specific $A_{1}$ adenosine receptor agonist (1 $\mu \mathrm{M}, 5 \mathrm{~min}, 3 \mathrm{~h}$ before the ischemic insult). The degree of neuronal damage was assessed by the trypan blue exclusion 
test. Exposure of the cultures ( $15 \mathrm{~min}$ before the exposure to R-PIA) to bisindolylmaleimide I (10 nM), a highly selective cell-permeable PKC inhibitor (selective for PKC $\alpha, \beta I, \beta I I, \gamma$ and $\varepsilon$ ), abrogated the protection conferred by R-PIA. By employing antibodies for p-PKC (PKC $\alpha$; Ser 657, PKC $\delta$; Ser 643 , PKC $\varepsilon$; Ser 729), we could demonstrate that exposure of the cultures to R-PIA resulted in activation (phosphorylation) of PKCE. Furthermore, insertion into the cells of PKCE translocation inhibitor peptide, resulted in abrogation of the protection conferred by R-PIA. These results demonstrate the involvement of activation of PKCE in the adenosine-induced protective signal transduction pathway in the neurons. A similar role for PKC $\varepsilon$ was shown before in the heart (Ping et al, Circ. Res., 88:59-62 [20011). Thus, PKC $\varepsilon$ appears to be a ubiquitous somatoprotective mediator in the adenosineactivated signaling cascade.

Keywords: adenosine,ilschemia, PKC, preconditioning

Mechanism of action of fast-onset antidepressant drugs Dremencov E. ${ }^{1}$, Gispan-Herman $\mathrm{I}^{1}$, Mendelman A. ${ }^{1}$, Overstreet D.H.' ${ }^{2}$ and Yadid G.

${ }^{1}$ Faculty of Life Sciences, Bar-Ilan University, Ramat-Gan; ${ }^{2}$ Dept. of Pychiatry, University of North Carolina, Chapel Hill, North Carolina, USA

In the last decade, a number of antidepressants were developed that demonstrated a more rapid onset of clinical effects than classical antidepressants. However, the mechanism that enables some drugs to have a faster onset of action than others is poorly understood. The aim of the current study was to determine dynamic changes in the brain in response to a fast-acting antidepressant to elucidate possible neurochemical parameters that correspond to the improvement of depressive behavior. In our previous studies, we found that Flinder sensitive line (FSL) rats, an animal model of depression, are characterized by: a) increased immobility during the forced swim test, b) absence of serotonin-induced dopamine release in the nucleus accumbens, and c) abnormal expression and increased inhibitory-like activity of $5-\mathrm{HT}_{2 \mathrm{C}}$ receptor. All of these parameters were normalized after chronic (14 day) treatment with antidepressants. In the current study, we found that subchronic (7 day) treatment with nefazodone (a fast-onset antidepressant), but not with despiramine (a classical antidepressant), normalized immobility time in the swim test in FSL rats. Nefazodone treatment of FSL rats also restored normal activity of the $5-\mathrm{HT}_{2 \mathrm{C}}$ receptor and accumbal serotonin-dopamine interaction after a subchronic treatment. We conclude that the restoration of accumbal serotonin-dopamine interaction via the $5-\mathrm{HT}_{2 \mathrm{C}}$ receptor seems to be a critical parameter for the fast onset of antidepressant treatment.

Keywords: antidepressant; fast onset; microdialysis; serotonin $5-\mathrm{HT}_{2 \mathrm{C}}$ receptor

\section{Effect of somatostatin on prostaglandin and nitric oxide} synthesis in glial cells

Dror N., Belinson H., Shemi D., Danon A. and

Fleisher-Berkovich $\mathrm{S}$

Dept. of Clinical Pharmacology, Faculty of Health Science,

Ben Gurion University P.O.B 4653, Beer-Sheva 84105

Recent evidence suggests that prostaglandins (PGs) are involved in the pathogenesis of Alzheimer's Disease (AD) and that nonsteroidal antiinflammatory drugs (NSAIDs) prevent its progression. Glial cells are a major source of PGs in the central nervous system (CNS) and are important in physiological and pathological processes in the CNS. Deficits in somatostatin (SS)-like and corticotropin releasing factor (CRF)immunoreactivity are recognized as prominent neurochemical deficits in AD patients. Fleisher-Berkovich et al showed that CRF can regulate PG synthesis in endothelial cells and fibroblasts (Endocrinology 136:4068-4072, [1995]). The aim of the present study was to investigate the role of SS in the regulation of basal and lipopolysaccharide (LPS)-induced PG production in primary rat glial cells culture. Our results show that SS $\left(10^{-9}-10^{-7} \mathrm{M}\right)$ inhibited basal PG production by $64 \%$ to $68 \%$. LPS increased in a dose and time dependent manner PG synthesis in rat glial cells. PG levels increased significantly after $24 \mathrm{~h}$ incubation with LPS. SS $\left(10^{-10} \mathrm{M}-10^{-9} \mathrm{M}\right)$ inhibited LPS-induced PG synthesis by $40 \%$ to $50 \%$.LPS increased NO release in a time and dose dependent manner. SS $\left(10^{-10}-10^{-9} \mathrm{M}\right)$ decreased LPS-induced NO release by $25-30 \%$. It is tempting to speculate that if brain PG and NO synthesis induced by inflammatory agents like LPS is significantly regulated by neuropeptides like SS, then specific preventive and therapeutic modalities may be devised to intervene with these mechanisms. Dr. Sigal Fleisher-Berkovich is a Kreitman Post-Doctoral fellow Keywords: somatostatin, prostaglandins, nitric oxide, glial cells, Alzheimer's disease
Effect of nerve on regenerating neuromuscular junctions in dystrophin-deficient mutant $(m d x)$ mice

Durrant A.R. ${ }^{1}$, Szabo M. ${ }^{2}$, Salpeter M.M..$^{2 *}$ and Anglister L. ${ }^{1}$ ${ }^{1}$ Dept. of Anatomy and Cell Biology, Hadassah Hebrew University Medical School, Jerusalem $91120 ;{ }^{2}$ Dept. of Neurobiology and Behavior, Cornell University, Ithaca, NY 14853-2702. Deceased.

Normal development of neuromuscular junctions (nmj's) depends on proper innervation of muscles. In mutant mice with X-linked muscular dystrophy $(m d x)$, the nmj's have fewer junctional folds, altered shape (grapelike), and accelerated turnover rate of acetylcholine receptors (AChRs). The present study aimed to determine whether innervation of $m d x$ muscles by normal nerve can correct the $m d x$ nmj deficits. We surgically interchanged sternomastoid muscles of $m d x$ and normal (wt) mice. Months later, AChRs were labeled with fluorescent $\alpha$-bungarotoxin (Bgt) or ${ }^{125} \mathrm{I}$-Bgt and the chimeric-nmj's were analyzed for fine-structure, shape and AChR number. EM examination revealed that in all cases the host nerve innervated the transplanted muscle, but when wt nerve innervated $m d x$ muscle, the nmj's developed folds more fully than when $m d x$ nerve innervated $w t$ muscle. Fluorescent microscopy showed that a significant fraction $(>30 \%)$ of the nmj's formed when $w t$ nerve innervated $m d x$ muscle resembled "normal" plate-like nmj's, whereas all nmj's in wt muscles innervated by $m d x$ nerve exhibited "dystrophic" grapelike shape. Furthermore, ${ }^{125}$ I-Bgt binding (reflecting AChR number) of the transplanted $m d x$ muscles innervated by $w t$ nerve became similar to their contralateral $w t$ control muscles In all cases, wt muscles showed robust staining with dystrophin-antibody, while $m d x$ muscles showed no dystrophin-immunoreactivity, indicating maintenance of the transplanted muscle genotype. Together, these findings suggest that the phenotype of the nerve determines the development of junctional folds, the total AChR number and the shape of the regenerating $\mathrm{nmjs}$

Supported by NIH NS 09315 (MS), NIH GM 07469 (AD), and Israel Sci. Found. 685/01 (LA)

Keywords: dystrophin, neuromuscular junction, sternomastoid muscle, acetylcholine receptor

Visuo-motor impairments in unilateral neglect Dvorkin A.Y. ${ }^{1,2}$, Flash T. ${ }^{2}$, Behrmann M. ${ }^{3}$, Soroker N. ${ }^{4}$ and Bentin S.

${ }^{I}$ The Interdisciplinary Center for Neural Computation, The Hebrew University, Jerusalem. Jerusalem; ${ }^{2}$ Dept. of Computer Science and Applied Mathematics. Weizmann Institute of Science, Rehovot 76100; ${ }^{3}$ Dept. of Psychology, Carnegie Mellon University, Pittsburgh, USA: ${ }^{4}$ Loewinstein

Rehabilitation Center, Ra'anana

In this study we investigated the spatial and temporal characteristics of the attentional deficit in unilateral neglect patients during reaching toward visual targets located within the horizontal plane, by using the double-step (DS) target displacement paradigm.

Results from two neglect patients showed significantly longer reaction times (RT) and movement times (MT), compared with control subjects. Moreover, a contralesional to ipsilesional gradient was found in both RT and MT.

The initial direction of motion in both young and elderly healthy controls depended on the modification time (RT Inter-Stimulus-Interval), and included averaged/non-averaged and direct trajectories. In contrast, both patients showed no such dependence and no averaged modified trajectories. These findings suggest the patients' inability to amend their ongoing movements in response to a sudden change in the target location. Furthermore, a higher proportion of direct trajectories was observed in DS movements, for movements that were initially directed toward the left side of space.

In addition, whereas in elderly subjects and patients, some of the DS movements contained a pause before the direction of the trajectory was modified, (15\% and $35 \%$ of the DS movements respectively), the patients displayed significantly longer pauses. Moreover, pauses were significantly longer when the first target appeared ipsilesionally, with respect to the trunk-midline. These findings reflect the existence of a competitive bias in favor of ipsilesional stimuli.

Results from a perceptual control experiment demonstrated the existence of a spatial perception deficit in both patients. Taken together, our findings suggest that the impairment seen in the two patients could not be related to either pure perceptual or pure motor deficits.

Keywords: neglect, reaching, competition 
A new member of the cyclin family may play a role in long-term memory

Edelheit S., Yahav S. and Meiri N.

Institute of Animal Science, Agriculture Research Organization, Volcani Center, Bet-Dagan 50250

Identifying the molecular correlates of long term-memory has been a focus of research, nevertheless little progress has been made and only few molecules have been directly correlated with the neuronal processes underlying consolidation. In this project we were interested in findings gene products that are altered hours after learning. Since the prevailing hypothesis emphasizes the role of neuronal plasticity in memory consolidation we used a behavioral paradigm that occur during early developmental stages and hens amplify changes in memory-related gene expression. We used the one-day old chick passive-avoidance learning paradigm in which a chick learns to avoid eating a bead coated with an unpleasant tasting substrate. The intermediate medial hyperstriatum ventrale (IMHV) is centrally involved in storing this memory

A screen using differential display for changes in mRNA expression in the IMHV between 2 and 24 hours after training chicks on the passive-avoidance memory task, revealed a new gene sequence with an homology of $80 \%$ with the cyclin family that was induced after training with a peak at 12 hours after training.

Our results coincide with earlier findings suggesting selective induction of a different member of the cyclin family - ania- 6 by different types of neurotransmitters in the striatum. It is also known that certain cyclins can interact with RNA polymerase II. This might be a novel mechanism for regulation of neuronal gene expression which convergent with multiple lines of evidence suggesting that acute regulation of pre-mRNA splicing is important in neuronal plasticity. Keywords: passive avoidance, cyclin, chicks, differential display

The extinction of fear conditioning in Medaka fish (Oryzias latipes)

\section{Eisenberg M. and Dudai Y}

Dept. of Neurobiology, Weizmann Institute of Science, Rehovot 76100

Ample evidence indicates that experimental extinction is learning rather than unlearning. However, it is yet unclear how different is the mechanism of extinction from that of acquisition. In the present study we used a developmental approach in order to address this question. Medaka fish of three different ages (1week, 4 week and 7 months) were subjected to fear conditioning. They were trained by pairing light (conditioned stimulus, CS) with electric shock (unconditioned stimulus, US) for 20 trials over 2 days. Fear response was evaluated by computerized comparison of locomotion before and during CS presentation. Subjects were tested on memory retention starting 24 hours after the last training trial. Afterwards, the fish were subjected to 20-30 successive trials over 2-3 days in the absence of the US. Significant age-dependent differences were unveiled in retention and extinction of conditioned response. Whereas all age groups readily acquired the task, the youngest fish (1 week old) failed to retrieve memory of learned fear 24 hours later. Adult fish (7 month old) successfully extinguished the fear response in 20 trials, while the 4 week-old fish continued to exhibit a startle response to the CS even after 30 trials. These results suggest that extinction demands a more mature nervous system than the acquisition of conditioned fear. In addition, the results validate the ability to use Medaka fish, which are a convenient neurogenetic model, for the study of the ontogeny of learning and memory.

Keywords: fear conditioning, extinction, acquisition, ontogenesis

\section{A novel amphiphilic motif in the $\mathrm{N}$-terminal helix of} heterotrimeric G-proteins

Elia N., Kosloff M. and Selinger Z.

Dept. of Biological Chemistry, The Hebrew University, Jerusalem

Heterotrimeric G-proteins relay signals between membrane-bound receptors and downstream effectors. The $\alpha$ subunits of this super-family are anchored to the membrane by one or more lipid modification at their $\mathrm{N}$-termini. These modifications can be palmitoylation, myristoylation or both. As no sequence determinant for palmitoylation is apparent, we used systematic homology modeling of all different human $\mathrm{G}_{\alpha}$ proteins to look for a three-dimensional structural determinant of palmitoylation, rather then a linear sequence motif.

Comparison of the $\mathrm{N}$-termini of this super-family revealed that all $\alpha$ subunits modified only by palmitoylation contain a similar structural motif at their N-terminal helix. This motif is characterized by a prominent basic patch that extends a positive potential well beyond the molecular surface of the protein. Furthermore, this structural motif is oriented opposite to the face that interacts with the $\beta \gamma$ subunits. Hence, these positive patches are free to interact with the negatively charged inner surface of the plasma membrane.

Based on previous results, we suggest that that efficient palmitoylation of $G_{\alpha}$ proteins requires prior targeting to the plasma membrane. The signal for this membrane localization can therefore be either myristoylation or the novel motif that we identified. This signal is cooperative with the interaction of the $\alpha$ subunit with the $\beta \gamma$ complex. The $N$-terminus of a $G_{\alpha}$ protein can therefore be described as amphiphilic, containing dual signals attracting it to the membrane and enabling it to undergo palmitoylation. As palmitoylation has been shown to modify a plethora of proteins extending beyond G-proteins, this motif could be widely applicable.

Keywords: structure, G-protein, membrane-attachment

Proteomics approach to study the rule of ERK in learning and plasticity

Elkobi A. ${ }^{1}$, Admon A. ${ }^{2}$. and Rosenblum K. ${ }^{1}$

${ }^{7}$ Faculty of Science and Science Education,University of Haifa; ${ }^{2}$ Dept. of Biology Protein Center, Technion, Haifa

We are interested in the role of the extracellular regulated kinase I/II (ERKI/II) in the formation of long-term memory and plasticity. ERK I/II is involved in both early and late phases LTP (Rosenblum et al. J. Neuroscience [2002]). In addition, ERKI/I activity is correlated and necessary for the formation of long-term memories (e.g. Berman et al., 1998). Using subcellular fractionation, phospho-specific antibodies and proteomics (two Dimensional Electrophoresis, mass spectrometry, and bioinformatics) we are trying to identify ERKI/II substrates in the brain that are involved in memory formation. In order to identify ERKI/II substrates we are using antibody that recognizes phosphorylated-Threonine only when followed by the amino acid Proline (the favorite phosphorylated site by ERKI/II). In addition, we test the modulation in synaptosomal protein expression and post translation modifications using different fractionation methods. The functionality of the identified spots or bands will be determined in the insular cortex both following learning (novel taste learning) and LTP.

Keywords: consolidation, ERK, proteomics

\section{Oxidative stress induced by 6-hydroxydopamine affects} ubiquitin-conjugates, protein degradation and proteasome activity in PC12 cells: Implications for the pathogenesis of Parkinson's disease

Elkon H., Melamed E. and Offen D.

Dept. of Neurology, Felsenstein Medical Research Center, Rabin Medical Center, Petah-Tikva, 49100, and Sackler School of Medicine, Tel Aviv University, Tel-Aviv doffen'a) post.tau.ac.il

Mutations in familial Parkinson's disease (PD) have been associated with the failure of protein degradation through the ubiquitin-proteasome system. Impairment of proteasome function has also been suggested to play a role in the pathogenesis of sporadic PD. Protein damage seen in the substantia nigra in PD was postulated to be induced by the local oxidative metabolism of dopamine. We examined the proteasome activity in PC12 cells treated with 6-hydroxydopamine (6-OHDA), the dopamine synthetic derivate used in models of $\mathrm{PD}$. We found that the treatment increased protein degradation, increased the levels of free ubiquitin and ubiquitin-conjugated proteins, in a dose dependent manner. In addition, there was an increase in proteasome trypsin-, chymotrypsin- and post acidic protease-like activities in cells treated with $10-100 \mathrm{uM}$ of 6-OHDA, whereas higher doses caused a dramatic decline. Similarly, the presence of $0.3 \mathrm{mM} 6-\mathrm{OHDA}$ for up to 10 hours increased proteasome activities while further incubation (10-24 hrs) reduced them markedly. We demonstrated that 6-OHDA treatment increased the protein degradation, accumulation of carbonyls groups and caspase- 3 activity, while addition of antioxidant, N-acetylcysteine, prevented these effects. In conclusion, our data indicate that mild oxidative stress elevates proteasome activities in response to the increase in protein damage. Severe oxidative insult may lead to failure of the ubiquitin system to clear defective proteins and cause protein aggregation and cell death. Control of protein clearance might offer a new strategy for therapy in neurodegenerative diseases in general and particularly for $\mathrm{PD}$

Keywords: Parkinson's disease, 6-hydroxydopamine,

ubiquitin-conjugates, protein degradation, proteasome activity 
Axotomy induced reversed microtubules polarity leads to the formation of a vesicles trap and the extension of a growth cone's lamellipodium

Erez H. ${ }^{1}$, Shapira E. ${ }^{1}$, Hoogenraad C.C. ${ }^{2}$, de Zeeuw C.I. ${ }^{2}$ Galjart ${ }^{3}{ }^{3}$ and Spira M.E.

Depts. of Neuroscience and Cell Biology ${ }^{2}$, Life Sciences

Institute, The Hebrew University of Jerusalem, Jerusalem;

${ }^{3}$ Erasmus MC, the Netherlands.

The transformation of a stable axonal segment into a motile growth cone is a critical step in the regeneration of amputated axons. A striking observation made in earlier studies from our laboratory, is that down stream, in the cascade leading to growth cone formation, anterogradely transported vesicles accumulate at a defined compartment some $100-150 \mu \mathrm{m}$ proximal to the tip of the cut axonal segment. This compartment forms the GC's center.

Here we began to explore the mechanisms by which vesicles are trapped at this specific location. To that end, we expressed EGFP-EB3 in cultured Aplysia neurons. EGFP-EB3 binds in stretches to the plus end of microtubules, moves with the growing MT's tips forming a comet tail like structure and thereby allows to image the dynamics and polarity of MTs. We found that axotomy leads to restructuring and reorientation of the MTs polarity at the cut axonal end. 100-150um from the cut axonal tip where the GC center is formed and vesicles are trapped, the MTs reorient such that plus ends point towards a common center- the trap. Proximally the trap is formed by MTs that maintain their original polarity. The distal boundary of the trap is formed by MTs with reversed polarity. Distal to that, following a short axoplasmic gap the MTs point their plus ends anterogradely.

We propose that the vesicles trap is formed by the reorientation of the MTs polarity thus directing molecular motors to deliver vesicles into the trap.

Keywords: axotomy, growth cone, microtubules, regeneration, EB3

ErbB4 receptor expression elevates following closed head injury

Erlich S. ${ }^{1}$, Shohami E. ${ }^{2}$ and Pinkas-Kramarski ${ }^{1}$

${ }^{T}$ Dept. of Neurobiochemistry. Tel-Aviv University, Tel-Aviv 69978; ${ }^{2}$ Dept. of Pharmacology, The Hebrew University, Jerusalem 91120

The ErbB-4 receptor tyrosine kinase and its ligand neuregulin are widely expressed in the nervous systems. To investigate their possible role in neurodegeneration we used the closed head injury (CHI) model. We demonstrate that levels of ErbB-4 are dramatically increased at the site of injury. ErbB-4 levels in the cortical region at the site of injury were significantly increased starting at day 3 post trauma, lasted two weeks and then declined to basal levels. Notably, the highest levels of ErbB-4 expression at the site of injury were observed one week after CHI. The staining patterns indicate that activated microglia/macrophages and neurons but not astrocytes, constitute the major population of cells that highly express the receptor at the injury site. Confocal microscopy analysis suggests that the high levels of ErbB-4 protein in activated microglia/macrophages is probably due to phagocytosis of neuronal cells. These findings indicate that ErbB-4 receptors may play a role in brain responses to head trauma.

Keywords: tyrosine kinase, ErbB-4, brain, closed-head injury, microglia/macrophages

\section{NRG mediates neuronal differentiation and survival in} PC12-ErbB4 cells

Erlich S., Goldshmit Y. and Pinkas-Kramarski R.

Dept. of Neurobiochemistry, Tel-Aviv University. Tel-Aviv 69978

Neuregulins (NRGs), a large family of transmembrane polypeptide growth factors, mediate various cellular responses depending on the cell type and receptor expression. To examine the possible role of ErbB-4 in neurons we have used the PC12 cell. Expression of ErbB-4 in PC12 cells demonstrated that NRG induced signals and neurite outgrowth, which were indistinguishable of those mediated by NGF. In PC12-ErbB-4 cells, NRG induced an initial weak mitogenic signal and subsequently neurite outgrowth. The differentiation induced by NRG involves generation of reactive oxygen species (ROS). In PC12-ErbB-4 cells NRG can rescue from apoptotic cell death induced by serum deprivation or TNFa treatment. We also show that NRG induces a significant protective effect from $\mathrm{H} 2 \mathrm{O} 2$ induced death. This effect of NRG is mediated by the PI3K signaling pathway, since NRG failed to rescue cells from $\mathrm{H} 2 \mathrm{O} 2$ insult in the presence of the PI3K inhibitor, LY294002. Furthermore, the downstream effector of
PI3K, PKB/AKT, is activated by NRG in the presence of $\mathrm{H} 2 \mathrm{O} 2$ and PKB/AKT activation is inhibited by LY294002. In addition, our results demonstrate that the high levels of ROS induced by $\mathrm{H}_{2} \mathrm{O}_{2}$, is inhibited by NRG. LY294002, which blocks NRG mediated rescue, increases ROS levels. Moreover. both $\mathrm{H} 2 \mathrm{O} 2$-induced ROS elevation and cell death are reduced by expression of activated PI3K. These results suggest that PI3K dependent pathways may regulate toxic levels of ROS generated by oxidative stress. In conclusion, our results demonstrate that neurite outgrowth induced by ErbB-4 in PC12 cells, requires MAPK and PKC signaling networks and NRG induced survival from apoptotic cell death requires PI3K signaling.

Keywords: phosphatidylinositol 3-kinase (PI3K), ErbB-4, neuregulin (NRG), tyrosine kinase, reactive oxygen species (ROS)

Expression of the proapoptotic protein ARTS in cerebellar granule cells in culture

Faber D. ${ }^{1}$, Loboda Y. ${ }^{1}$, Larisch S. ${ }^{2}$ and Finberg, J.P.M. ${ }^{1}$

${ }^{1}$ Dept of Pharmacology, Rappaport Family Faculty of Medicine, Technion, Haifa; ${ }^{2}$ Dept. of Pathology, Rambam Medical Center, Haifa

The protein ARTS (Apoptosis Related protein in the TGF $\beta$ Signaling pathway) is involved in apoptosis induced by TGF $\beta$ and by other pro-apoptotic factors. In mouse brain, ARTS was seen in a number of brain areas, including several parts of the cortex, hippocampus, septal nuclei and bed nuclei of stria terminalis.

In this work we explored the expression of AR'TS in cerebellar granule neurons in primary culture by immunohistochemistry. Content of ARTS increased rapidly after plating. After $24 \mathrm{~h}$ in vitro, more than $90 \%$ of the neurons were strongly ARTS positive. The number of ARTS positive neurons was similar in cultures grown in medium containing a physiological concentration of potassium, and in $25 \mathrm{mM} \mathrm{K}^{+}$medium (in which the cells are viable for a longer period in vitro). ARTS protein was seen to a similar extent in mitotic and non-mitotic neurons, as detected by BrDU incorporation. ARTS was detected by confocal microscopy in mitochondria and nuclei both in young $(24 \mathrm{~h})$ and mature (10 days in vitro) cultures. Since our previous work in COS cells has shown that migration of ARTS from mitochondrion to nucleus occurs at the onset of apoptosis, this finding may indicate which neurons in the culture are undergoing apoptosis. The finding of a high content of ARTS in cerebellar granule cells in culture contrasts with the near absence of ARTS in cerebellum of the post-natal rat as seen in sections of whole brain, and also correlates with the high degree of apoptotic cells ( $10 \%$ by TUNEL stain) in the neuronal culture in vitro

Keywords: apoptosis, ARTS, cerebellar granule cells

Transgenic excess AChE exerts non-catalytic effects on hippocampal LTP via PKC

Farchi N., Hochner B. and Soreq $\mathrm{H}$

Life Sciences Institute and Interdisciplinary Center for Neural Computation, Hebrew University. Jerusalem

AChE is known for its catalytic ACh hydrolyzing features, but most interestingly acts as a sensitive marker for acute and chronic stress, as a specific splice variant of $\mathrm{AChE}$ is highly elevated under such insults. Transgenic models for chronic expression of either the stress-related AChE (AChE-R) or its primary synaptic variant (AChE-S) express both altered LTP patterns in hippocampal CAl synapses and memory and learning impairments. Specifically, LTP is enhanced under transgenic excess of AChE-R ( $172 \%$ vs. $138 \%$ in WT) whereas excess AChE-S leads to normal levels of potentiation that dramatically decays to $37 \%$ within $3 \mathrm{~h}$ post-induction (vs. no decay in WT). Intriguingly, the decay phenotype was not rescued by either physostigmine $(1 \mu \mathrm{M})$ or carbachol $(0.5 \mu \mathrm{M})$ administration. Moreover, it was also evident in the hippocampus of transgenics expressing catalytically inactive AChE-S suggesting that non-catalytic features of $\mathrm{AChE}$ are involved in LTP maintenance. We examined the possible involvement of PKC in the distinct LTP responses of AChE-R or $-\mathrm{S}$ transgenic slices. Phorbol di-butyrate $(5 \mathrm{nM})$. a PKC activator, highly facilitated synaptic field potentials in AChE-R slices $(101 \%$ vs. $65 \%$ in WT) contrasted by poor $(30 \%)$ facilitation in AChE-S slices. Furthermore, a tetanus following PKC activation induced stable late-phase LTP in AChE-S slices, thus rescuing the LTP decav. To conclude. we report the contribution of $\mathrm{AChE}$ non-catalytic features involving $\mathrm{PKC}$ activity and/or levels in LTP priming and maintenance. This study may demonstrate stress-related modulation of neuronal plasticity. 
The physiological relevance of the physical interaction of voltage gated $\mathrm{K}^{+}$channels with protein components of the exocytotic machinery in PC12 cells

Fili O., Singer-Lahat D., Chikvasgvili D. and Lotan I Dept. of Physiology \& Pharmacology, Sackler Faculty of Medicine, Tel Aviv University, Tel Aviv 69978

Presynaptic voltage-gated $\mathrm{K}^{+}(\mathrm{Kv})$ channels play a physiological role in the regulation of transmitter release by virtue of their ability to shape presynaptic action potentials. However, in a previous work (Fili et al, 2001) we showed that in brain synaptosomes and Xenopus oocytes Kvl.1 channels and SNAREs proteins display a physical interaction. Also, our preliminary results show that another $\mathrm{Kv}$ channel, Kv2.1, also interacts with the SNARE proteins in oocytes. In this study we aimed to attribute a physiological role to the physical interactions of these $\mathrm{Kv}$ channels with the exocytotic proteins using the cracked PC12 cell assay (Hay and Martin 1992). Both channels, Kv1.1 and Kv2.1, are expressed in PC12 cells. In co-immunoprecipitation experiments we could observe a physical interaction between the SNARE proteins and the Kv2.1 channel in PC12 cells. We introduce GST-proteins corresponding to parts of the Kv1.1 and Kv2.1 fragments of the two channels that were shown in in vitro binding assays to either bind or not SNAP-25 or syntaxin 1A, and assayed their effects on the $\mathrm{Ca}^{2+}$ regulated neurotransmitter secretion in this system. Our results are consistent with the notion that the physical binding of $\mathrm{Kv}$ channels to the SNARE proteins modulates $\mathrm{Ca}^{2+}$ regulated neurotransmitter secretion

Keywords: Kv1.1, Kv2.1, SNARE proteins, PC 12

The endogenous cannabis system: role in the stress response and critical function in milk ingestion and survival in newborn mice

Fride E. . Foox A. ${ }^{1}$, Weidenfeld J. ${ }^{2}$, and Mechoulam R ${ }^{3}$ ${ }^{7}$ Dept. of Behavioral Sciences, College of Judea and Samaria, Ariel: ' Hadassah Medical School and ${ }^{3}$ Dept. of Medicinal Chemistry and Natural Products of the Hebrew University of Jerusalem

The ability of cannabis to stimulate appetite has been used to benefit patients suffering from malnutrition. Endogenous cannabinoids ("endocannabinoids") are found in brain peripheral organs, and maternal milk; they activate cannabinoid (CB1-CB2) receptors. We showed recently that $\mathrm{CB} 1$ receptors are critical for milk ingestion and survival in newborn mice. Here, we further investigate the requirement for $C B 1$ receptors in newborn feeding and development in CB1 receptor-knockout $\left(\mathrm{CB}^{-1}\right)$ mice. Milk intake, weight gain, and development were recorded in $\mathrm{CB}^{-1-}$ and in wild type pups. Further, pups were injected with a $\mathrm{CBI}$ receptor antagonist (SR 141716A) on day 1 of life

$\mathrm{CB}^{-/-}$pups did not ingest milk on the first day of life, and survival was significantly lower compared to wild types. SR 141716 A completely inhibited milk intake in wild type pups and resulted in almost $100 \%$ mortality within 7 days. The antagonist partially affected milk ingestion, growth and survival in $\mathrm{CB}^{-1-}$ pups.

Throughout this study, we noticed a greater vulnerability and mortality in $\mathrm{CB}^{--}$mice, suggesting a role for the endocannabinoid system in stress. Indeed, acute stress $(4 \mathrm{~min}$ noise) induced a rise in corticosterone and in ACTH in the sera of $\mathrm{CB}^{-1}$ mice, which was respectively, twice and 4 times higher in knockout compared to wild type mice.

We conclude that 1) neonatal milk ingestion is entirely dependent on the endocannabinoid-receptor system. A major role is played by $\mathrm{CBI}$ receptors; however our data support evidence for an additional ("CB3") receptor. 2) The cannabinoid system plays an important role in the response to stress

Keywords: cannabinoids, stress, development, feeding

T-cells express a functional ionotropic glutamate receptor GluR3, and glutamate by itself directly activates T-cell function.

Ganor $\mathrm{Y}^{1}$, Besser M. ${ }^{1}$ and Levite $\mathrm{M}^{1,2}$

${ }^{\prime}$ Dept. of Neurobiology. Weizmann Institute of Science Rehovot 76100: ${ }^{2}$ The Sackler Faculty of Medicine, Tel-Aviv University, Tel-Aviv 69978

T-cells may encounter glutamate, the major excitatory neurotransmitter in the nervous system, when patrolling the brain, and in glutamate-rich peripheral organs. Moreover, CNS glutamate levels increase in various pathological conditions in which T-cells may play a beneficial or detrimental role. Do T-cells express ionotropic glutamate receptors? Can glutamate by itself trigger T-cell function?

We found, for the first time, that normal human T-cells, human T-leukemia line, and mouse autoimmune anti - myelin - basic
- protein T-cells express high levels of specific ionotropic glutamate receptor of the AMPA subtype 3 (GluR3)

The evidence for GluR3 expression in T-cells include GluR3-specific RT-PCR and sequencing, showing identity to brain GluR3, western-blotting, and GluR3 cell-surface expression revealed by immunocytochemical - fluorescence staining and flow - cytometry.

Glutamate $(10 \mathrm{nM})$, in the absence of any additional molecule, activated specific T-cell functions, among them integrin dependent adhesion to laminin and fibronectin, and chemotactic - migration towards the chemokine SDF-1

AMPA receptor-agonists mimicked glutamate-induced effects, and the specific antagonists CNQX and NBQX blocked it

Taken together, we suggest that GluR3 expression in T-cells and glutamate-triggered T cell function could be important to numerous physiological and pathological conditions, and especially relevant to: a) T-cell transmigration to the CNS across laminin-containing blood-brain-barrier; b) T-cell mediated multiple sclerosis, c) Some human epilepsies in which specific anti-GluR3- antibodies are found and suspected to play a neurotoxic role. Based on our finding we speculate that granzyme-B-producing T-cells can cleave the GluR3B autoantigen in an autocrine (from their own surface-expressed-GluR3) or paracrine (from neighboring T-cells) fashion, not only from neuronal cells, as suggested thus far.

Keywords: glutamate, ionotropic-glutamate-receptor GluR3,

T-cells, neuroimmunomodulation, autoimune-epilepsy

Mechanisms of aging of the growth hormone-releasing hormone receptor

Gaudreau P ${ }^{1,2}$, Robinette K. ${ }^{1}$, Veyrat-Durebex C. ${ }^{1,3}$, Strecko $\mathrm{J}{ }^{1}$. Ferland $\mathrm{G}$. ${ }^{3}$ and Farrajota $\mathrm{K}$.

${ }^{2}$ Laboratory of Neuroendocrinology of aging, CHUM Research Center, Notre-Dame Hospital and departments of ${ }^{2}$ Medicine and ${ }^{3}$ Nutrition, University of Montreal, Montreal, Canada.

In aging mammals, a decreased responsiveness of somatotrophs to growth hormone-releasing hormone (GHRH) leads to low GH and IGF-I serum levels. This event was proposed to be responsible for a diminution of muscle mass, increase adiposity and deterioration of several tissues and organs. In the anterior pituitary, it has been related to changes in the level of GHRH-receptor (R) mRNA transcripts and immunoreactivity and to modifications of GHRH binding parameters. In long-term moderate calorie-restricted rats, a model of successful aging, youthful levels of pituitary GHRH-R mRNA transcripts and GHRH binding sites are maintained. Among regulatory factors positively impacting on GHRH-R, corticosterone and testosterone could be proposed as candidates. In 18-month-old calorie-restricted rats, serum corticosterone and testosterone are increased when compared to 18-month-old ad libitum-fed rats. An optimal control of glycemia, such as in caloric restriction, could also protect the GHRH-R. In streptozotocin-diabetic rats, levels of pituitary GHRH-R mRNA transcripts exhibit several disturbances according to the length and severity of diabetes. Moreover, a glucotoxicity state mimicking that found in aged or diabetic rat serum down-regulates the human GHRH-R, stably expressed in BHK cells, indicating that it may represent a common mechanism of GHRH-R aging. Altogether, these results suggest that circulating steroids and glucose may be critical to regulate the expression of the pituitary GHRH-R in aging. As seniors represent the fastest growing segment of the population in industrialized countries, identification of cellular and molecular mechanisms involved in somatotroph axis dysfunction may allow to design new interventions to delay somatopause.

Linking Lissencephaly type I genes to MAPK pathway Gdalyahu A., Ghosh I., Levy T. and Reiner O. Dept. of Molecular Genetics, Weizmann Institute of Science, Rehovot 76100

Lissencephaly type I is a severe human disease that affects $1 / 30,000$ live births. It is characterized by a relatively smooth cerebral surface, and an abnormal organization of the cortical layers. This is the result of an abnormal embryonic neuronal migration. The clinical manifestations are short life span, epilepsy and mental retardation. Mutations in LISI and doublecortin (DCX) have been shown to cause Lissencephaly type I. A third gene, doublecortin-like kinase (DCLK) is thought to participate in the DCX pathway. Known protein interactions of LISI and DCX suggest their involvement in intracellular transport.

Intracellular transport is a complicated problem for a highly polar cell like a neuron. A recent idea suggests that it would be advantageous for a neuron to assemble the components of a 
signaling pathway by loading them together on a scaffold protein. This scaffold protein is bound to a motor protein, and thus delivers the entire complex to the right place. This can ensure that the components of a pathway will be in the right ratio, all together, and promise spatial regulation on the pathway. According to this model, proteins of the same pathway are predicted to be transported together.

Here we show that both DCX and DCLK interact with a scaffold protein that assembles the mitogen activated protein kinase (MAPK) pathway in neurons.

This observation links for the first time neuronal migration with the MAPK pathway. Our hypothesis is that these interactions function to regulate the localization of MAPK module.

Keywords: lissencephaly, doublecortin, microtubule, neuronal migration

\section{The association of DRD4 and 5-HTTLPR with infant temperament}

Geller V., Auerbach J., Belmaker RH., Ebstein R. and Levine J. Beer Sheva Mental Health Center, Psychiatry Research Unit, Ben Gurion University of the Negev, Beer-Sheva

It was previously shown that the dopamine D4 exon III repeat (D4DR) and the serotonin-transporter promoter region (5-HTTLPR) polymorphisms are not only associated with adult personality traits but also with temperament in 2 -week old neonates. We now report the results of a third study of these infants and their temperament at 12 months. This study examined the association between two common polymorphisms, the DRD4 gene and the 5-HTTLPR and temperament in 12-month-old infants 22 infants had a least one copy of the 6-8 repeat DRD4 alleles (L-DRD4) and 39 had two copies of the 2-5 repeat allele (S-DRD4). 20 infants were homozygous for the short form $(\mathrm{s} / \mathrm{s})$ of 5 -HTTLPR while 41 were either heterozygous for the short and the long form (1/s) or were homozygous for the long form $(1 / 1)$. The infants were observed in a series of standard temperament episodes that elicited fear, anger, pleasure, interest, and activity. L-DRD4 infants showed less interest in a structured block play situation and more activity in a free play situation. They also displayed less anger in an episode of mild physical restraint. Infants with s/s 5-HTTLPR showed less fearful distress to stranger approach and less pleasure in a structured play situation than infants with $1 / 1$ or $1 / \mathrm{s} 5$-HTTLPR. Duration of looking during block play was affected by a significant interaction between DRD4 and 5-HTTLPR. Shortest duration of looking was associated with the L-DRD4 and s/s 5-HTTLPR genotypes. These results support previous data showing that the origins of the molecular control of human temperament lie in infancy. Keywords: dopamine D4 receptor gene, serotonin transporter promoter genes, infant temperament

\section{DCX and development}

Ghosh I., Gdalyahu A., Levy T., Caspi M. and Reiner O Dept. of Molecular Genetics, Weizmann Institute of Science. Rehovot 76100

The Lissencephaly syndromes in humans involve abnormal cortical lamination and are medically categorized as neuronal migration defects. Two genes involved in Lissencephaly type I have been identified: LISI and DCX.

Doublecortin (DCX), a microtubule binding protein, maps to the $\mathrm{X}$ chromosome and mutations in this gene results in Lissencephaly in males or subcortical heterotopia in females. This strongly suggests a role for this gene product during neuronal migration. The protein consists of two 80 residue evolutionary conserved repeats and a serine proline rich C-terminal region. The repeats bind microtubules and most mutations that are found in Lissencephaly patients cluster in this domain The C-terminal portion has been shown to interact with clathrin adapter proteins. AP-1 and /or AP-2, thus suggesting a potential role of DCX in protein sorting or vesicular trafficking.

Scaffold proteins have been shown to regulate MAP kinase related pathwavs

We have identified a specific MAP kinase which phosphorylates DCX which maybe important during neuronal migration.

We have identified the phosphorylation sites on DCX and have also identified the regions through which it interacts with the MAP kinase complex. Using different biochemical approaches we have established that DCX is a key molecule in neuronal migration.

Keywords: DCX, lissencephaly, MAP kinase, phosphorylation
External barium inhibits inactivation gating of KCNQ1 channels

Gibor G., Peretz A. Schottelndreier H. and Attali B

Dept. of Physiology and Pharmacology, Sackler School of Medicine, Tel Aviv University, Tel Aviv 69978

The KCNQ1 pore-forming $\alpha$ subunit belongs to a newly characterized $\mathrm{K}^{+}$channel family, KCNQ, which forms voltage-gated $\mathrm{K}^{+}$channels. Here we used $\mathrm{Ba}^{2+}$ ions to probe the permeation pathway of homomeric KCNQ1 channels. In addition to its voltage-dependent block of the pore, $\mathrm{Ba}^{2+}$ also alters the gating of homomeric KCNQ1 channels by favoring the closed state. $\mathrm{Ba}^{2+}$ produces a marked rightward shift of the voltage-dependence of activation $(+33 \mathrm{mV})$ and an acceleration of the deactivation kinetics. However, $\mathrm{Ba}^{2+}$ also reduces channel inactivation as revealed by the suppression of the hook of the tail current, an additional effect that opposes KCNQ1 current inhibition. To further investigate the effect of $\mathrm{Ba}^{2+}$ on KCNQ1 inactivation, we used the L273F KCNQ1 mutant. This naturally occurring mutation, located in the transmembrane segment $\mathrm{S} 5$ produces macroscopic inactivation. $\mathrm{Ba}^{2+}$ potently inhibits the maximum conductance and the macroscopic inactivation of the L273F KCNQ1 mutant. In high external $\mathrm{K}^{+}$ the impact of $\mathrm{Ba}^{2+}$ on channel gating is relieved. At $50 \mathrm{mM}$ external $\mathrm{K}^{+}, \mathrm{Ba}^{2+}$ neither shifts the voltage-dependence of activation nor accelerates deactivation kinetics of both WT and L273F KCNQ1 channels. Similarly, $\mathrm{Ba}^{2+}$ loses its ability to prevent channel inactivation in $50 \mathrm{mM} \mathrm{K}^{+}$. Interestingly, pore block by $\mathrm{Ba}^{2+}$ was poorly affected by $50 \mathrm{mM}$ external $\mathrm{K}^{+}$, suggesting that $\mathrm{Ba}^{2+}$, like $\mathrm{K}^{+}$ions, may interact with the channel pore at more than one site. Though the mechanisms underlying the gating effects of $\mathrm{Ba}^{2+}$ are unknown, we suggest that they are coupled to the permeation process.

Keywords: permeation, gating, $\mathrm{K}+$ channels

Characterization of the antiproliferative activity of phenothiazines in neuroblastoma and in glioma cell-lines. Relevance to brain cancer therapy
Gil-Ad I. and Weizman A

${ }^{1}$ Laboratory of Biological Psychiatry, FMRC, Campus Rabin and Geha Mental Health Center, Petah-Tiqva 49100;

${ }^{2}$ Shalvata Mental Health Center and Tel-Aviv University

Recent studies in our laboratory revealed that some phenothiazines possess a potent eurotoxic and antiproliferative activity in different neuronal cultures.

The aim of this study was to characterize the antiproliferative activity of different phenothiazines in human neuroblastomas (SK-N-SH) and (SHSY-5Y) and rat glioblastoma (C6) cell-lines. Chlorpromazine, perphenazine, fluphenazine and thioridazine induced a potent cytotoxic activity in all the lines (IC50 10-15mcM). Thioridazine showed the most potent effect. Thioridazine, but not clozapine, a non phenothiazine tricyclic neuroleptic, induced DNA laddering in rat glioma and in human neuroblastoma cell-lines. Flow cytometry of neuroblastoma and glioma cells stained with propidium iodide and treated with the different phenothiazines $(10-40 \square \mathrm{mcM})$ showed a dose dependent increase in the fragmented DNA (up to $80.0 \%-90 \%$ vs $1-5 \%$ in controls). Analysis of the cell cycle of the neuroblastoma cells treated with low concentrations (1-20mcM) of perphenazine revealed a dose dependent increase in the Gl, and a decrease in the S phase, suggesting inhibition of DNA synthesis. Determination of the Caspase -3 activity in the neuroblastoma cells treated with thioridazine showed a marked dose dependent increase in the activity $4 \mathrm{hr}$ after drug exposure. Glioma (C6) cells co-stained with propidium iodide and hoechst reagents showed the characteristic reddish apoptotic bodies in the nuclei of the phenothiazine treated cells.

Conclusions: Phenothiazines possess a dramatic cytotoxic activity in brain derived tumors. The effect of phenothiazines is associated with DNA fragmentation, arrest of cell cycle, and caspase 3 activation. We suggest that some cytotoxic neuroleptics could play a role in the therapy of brain derived tumors.

Keywords: phenothiazines, brain tumors, apoptosis

Lithium modulates neuron survival and glial growth in culture - involvment of polyamines

Gilad G.M. and Gilad V.H.

Research and Development, Laboratory of Neuroscience, Assaf Harofeh Medical Center, Zrifin 70300

Lithium, the most prevalent treatment for manic-depressive illness, may have neuroprotective effects after brain injury. In young primary cultures, lithium can exert neurotoxic effects associated with reduction in polyamine synthesis, but neuroprotective effects as cultured neurons mature. Evidence 
indicating that lithium can affect glial cells directly, leads us to postulate that lithium may exert some of its effects on neurons indirectly, via glial cells. Here we used rat cerebellar cultures to ascertain the effects of lithium $(\mathrm{LiCl})$ on the activity of ornithine decarboxylase (ODC, the enzyme catalyzing the first limiting step in polyamine synthesis) and on neuron survival, and to compare the effects of lithium with those of the ODC inhibitor, $\alpha$-difluoromethylornithine (DFMO). Additionally, we examined the effects of lithium and DFMO on cultured cerebellar glial cells (astroglia and microglia). Switching cultures from high $(25 \mathrm{mM})$ to low $(5 \mathrm{mM}) \mathrm{K}^{+}(\mathrm{KCl})$ medium concentrations served as the traumatic insult. The results indicate that: 1) While high, depolarizing $\mathrm{K}^{+}$concentration enhances neuron survival, it inhibits astroglial growth. 2) $\mathrm{LiCl}$ $(1-8 \mathrm{mM})$ enhances neuronal survival, but inhibits astroglial growth in cultures switched from high to low $\mathrm{K}^{+}$. 3) $\mathrm{LiCl}$ treatment leads to reduced ODC activity. 4) DFMO enhances neuron survival, but inhibits astroglial growth in cultures switched to low $\mathrm{K}^{+}$medium. 5) $\mathrm{LiCl}$ or DFMO treatment of confluent glial cultures lead to transformation of astroglia from epithelioid (flat) to stellate morphology and to increased numbers of microglia. 6) Combined $\mathrm{LiCl}$ and DFMO treatment is highly cytotoxic to both neurons and glia in culture. The findings indicate that lithium can exert direct effects on astrocytes, as reflected by growth retardation and altered cell morphology of cultured astroglia, and suggest that these effects may be associated with inhibition of polyamine synthesis. Moreover, the results imply that direct effects on astrocytes and microglia may contribute to the neuroprotective effects of lithium.

Keywords: astrocytes, alpha-difluoromethylornithine,

microglia, neuroprotection, ornithine decarboxylase, rat

Funcional recovery by lysyl oxidase inhibitor treatment after spinal cord injury in rodents

Gilad V.H. and Gilad G.M

Research and Development, Laboratory of Neuroscience, Assaf Harofeh Medical Center, Zrifin 70300

Lysyl oxidase (LO), an extracellular matrix (ECM)-forming enzyme was recently implicated in modulating the ECM and in scar formation at CNS injury sites. Modulations of the ECM and scar formation at CNS injury sites are considered prohibitive for successful axon regeneration, thus restricting functional recovery. Therefore, inhibition of LO may be conducive for regeneration and recovery of function. Previously, we found evidence that active extracellular LO molecules appear in a spatiotemporal manner in sites of CNS injury and that treating adult mice with the LO inhibitor $\beta$-aminopropionitrile (BAPN) can result in accelerated and more complete functional recovery after spinal cord injury. In the present study, adult rats received daily treatment of BAPN (100 mg/kg, intraperitoneally) for 20 days after complete unilateral spinal cord transection. This treatment led to an accelerated and more complete functional recovery as compared to controls. Additionally, in contrast to controls, which showed relatively minor functional deficit after a second transection, produced at 90 days at the site of the first spinal cord injury, BAPN rats demonstrated functional deficits and a functional recovery time course similar to those observed after the first injury. The mode of functional recovery of BAPN-treated rats suggests that axonal regeneration of long descending tracts may have occurred. These and previous findings indicate that BAPN, by inhibiting LO, may enhance recovery after spinal cord injuries.

Keywords: $\beta$-aminopropionitrile, extracellular matrix

regeneration, rat

\section{A novel thiol antioxidant that can cross the blood brain barrier demonstrates neuroprotective effects in a mice model of MOG-induced chronic experimental autoimmune} encephalomyelitis

Gilgun-sherki Y ${ }^{1}{ }^{2}$ Panet $\mathrm{H}^{1}{ }^{1}$, Livnat A. ${ }^{3}$, Melamed E. ${ }^{1}$, Offen $\mathrm{D}$ and Atlas D

${ }^{I}$ Dept. of Neurology, Felsenstein Medical Research Center, Rabin Medical Center, Petah-Tikva, 49100, and Sackler School of Medicine, Tel Aviv University, Tel Aviv 69978; 'Institute of Life Sciences, Edmond Safra Campus Givat Ram, Jerusalem. 91904; ${ }^{3}$ Novia Pharmaceuticals Ltd. P.O.Box 10209 Tel Aviv 61101 doffen a post.tau ac.il

Oxidative stress is believed to play a crucial role in the initiation and enhancement of the inflammation process that lead to demyelination in multiple sclerosis. We examined whether treatment with $\mathrm{N}$-acetylcysteine-amid (AD4), a novel thiol-antioxidant that readily crosses the blood brain barrier might provide a beneficial effect in the chronic, experimental autoimmune encephalomyelitis (EAE) model induced by myelin oligodendrocyte glycoprotein (MOG). Mice (C3H.SW, $\mathrm{n}=15)$ were injected s.c. twice with $\mathrm{pMOG} 35-55(3 \mathrm{mg} / \mathrm{kg}$ on day 1 and 8$)$ to induce EAE. AD4 treatment $(0.2 \mathrm{~g} / \mathrm{kg} \times 2 \mathrm{i} . \mathrm{p}$. and $0.3 \mathrm{~g} / \mathrm{kg}$ in drinking water daily) started in three groups on days 1,8 and at day 14 after the appearance of the symptoms. We found that while most of the control MOG-treated mice developed significant clinical manifestations with complete hind limb paralysis, the AD4-treated mice were resistant when treated from day 1 and showed only very mild and delayed clinical signs when treatment started at day 8 or 14 Histological examination of spinal cord sections showed, in the MOG-injected group (in the absence of AD4), multifocal areas of pervascular lymphohistocytic inflammation containing activated macrophages associated with loss of myelin. In contrast, in the AD4-treated animals, only mild foca inflammatory changes were observed. Similarly, immunostaining and silver staining of the neurons indicated a massive axonal damage in the EAE control group, and only a minimal damage was seen in the AD4-treated groups. In conclusion, our study demonstrates that in a chronic EAE model, administration of a BBB penetrating thiol-antioxidant prior to or even after the appearance of symptoms, significantly reduces the induced axonal damage, motor dysfunction and death.

Keywords: multiple sclerosis, experimental autoimmune encephalomyelitis (EAE), Myelin oligodendrocyte glycoprotein (MOG), AD4

Glatiramer acetate neuroprotective effects in a C57/b mice model of MOG-induced chronic experimental autoimmune encephalomyelitis

Gilgun-sherki Y., Panet H., Holdengreber V., Melamed E. and Offen D.

Dept. of Neurology, Felsenstein Medical Research Center Rabin Medical Center, Petah-Tikva, 49100, and Sackler School of Medicine, Tel Aviv University, Tel-Aviv 69978 doffen'apost.tau ac.il

Glatiramer acetate (GA, COP-1) is effective in the treatment of relapsing-remitting multiple sclerosis. Here we examined whether treatment with $\mathrm{GA}$ might provide a beneficial effect in the chronic experimental autoimmune encephalomyelitis (EAE) model induced by myelin oligodendrocyte glycoprotein (MOG). Mice $(\mathrm{C} 57 / \mathrm{b}, \mathrm{n}=20)$ were injected s.c. twice with pMOG $35-55(12 \mathrm{mg} / \mathrm{kg} \times 2)$ and $\mathrm{GA}(25 \mathrm{mg} / \mathrm{kg} \times 2$ s.c. $)$ or saline (control group), on day 1 and 8. Control MOG-treated mice developed significant clinical manifestations with complete hind limb paralysis, while the GA-treated mice showed only very mild and delayed clinical signs (clinical score $2+/-0.3$ vs. $0.4+/-0.2, p<0.001$ ). GA treatment provided constant resistance following MOG injection at least for 50 days until the animals were sacrificed. Histological examination of CNS tissue sections showed multifocal areas of perivascular lymphohistiocytic inflammation with loss of myelin in the control mice. By contrast, in the GA-treated animals, there were only mild focal inflammation changes. In parallel, silver staining (Bielshowsky) of the neurons indicated massive axonal damage in the EAE control group, while there was only minimal damage in GA-treated mice. Antibodies against amyloid precursor protein used to measure axonal damage demonstrated lower injury response, as measured by the Image-j software $(65 \% \mathrm{p}<0.001)$. In conclusion, our study demonstrates that in a chronic EAE model, GA immunization attenuates the induced axonal damage.

Keywords: multiple sclerosis, experimental autoimmune encephalomyelitis (EAE), myelin oligodendrocyte glycoprotein (MOG), axonal damage, glatiramer acetate

Radial correlation MRI contrast for global neuronal synchronization: Observation of cortical layers during spontaneous activity

Goelman G.

MRMRS lab. The Human Biology Research Center, Dept. of Medical Biophysics and Nuclear Medicine, Hadassah Hebrew University Hospital, Jerusalem

A new magnetic resonance imaging (MRI) post-processing contrast named 'Radial Correlation Contrast $(R C C)$ is described. The contrast gives the average communication for each volume element with its surroundings and its direction for a chosen distance. The method identifies dynamic structures based on their temporal fluctuations. In neuro-imaging these fluctuations are shown to be generated in the capillary bed, thus presenting neuronal activity. While the average communication shows good gray/white contrast, its direction is more sensitive and accurate for dynamic structural definition. It is shown that during spontaneous activity, short-range communication identifies specific structures. such as cortical layers. The agreement between these $R C C$ structures and 
cortical layers obtained by histology is excellent. Longer-range communication clearly identifies major cerebral structures and has the potential of showing their interaction during specific brain activity. This non-invasive ability to follow neuronal communication at various distances will enable a better understanding of brain function.

Keywords: fMRI, neuronal synchronization, cortical layers

NGF and BDNF regulation in the brains of interleukin-1-knock-out mice Golan $\mathrm{H}^{1,3}$, Shalom R. ${ }^{1}$ and Huleihel M. ${ }^{2}$ ${ }^{7}$ Dept. of Developmental and Molecular Genetics, ${ }^{2}$ Dept. of Immunology, Faculty of Health Sciences and Zlotowski Center of Neuroscience, Ben-Gurion University, Beer-Sheva

The neurotrophic factors, NGF and BDNF are involved in neuronal survival, morphogenesis and modulation of synaptic strength in the adult brain. Both factors are differentially regulated and their levels vary between different brain regions. Activation of interleukin-1 (IL-1) was implicated in the regulation of NGF and BDNF. Using the IL-1 knock-out (KO) $\mathrm{balb} / \mathrm{c}$ mice we have examined the possibility that basal levels of IL-1 may control NGF and BDNF protein expression. Brain tissue of IL-la-KO, IL-lb-KO, IL-lab-KO, IL-1Ra-KO and wild-type (WT) were analyzed. BDNF levels were higher and more variable than NGF in all examined brain areas; cerebral cortex, thalamus, cerebellum and hippocampus. No significant difference was observed in both NGF and BDNF levels between WT and KO mice in all examined brain regions. Nevertheless, BDNF/NGF ratio was constant in each tested region in balb/c mice; $5.2 \pm 2$ in the cerebral cortex, $4.5 \pm 3$ in the cerebellum and $3.7 \pm 1$ in the hippocampus. All IL-1-KO mice to the same background preserve the ratio observed in the comparable brain region of the WT mice. In contrast, no relation between BDNF and NGF levels was detected in JBC-57 WT mice. However, ILlb-KO on JBC-57 background exhibit in the cerebral cortex a constant ratio of BDNF/NGF similar to that observed in this area in the balb/c KO and WT, which is significantly different from its control. Our data suggest that the involvement of IL1 in BDNF/NGF regulation is distinct between mice strains. In the JBC-57 mice the basal levels of IL-1b may be implicated in the regulation of NGF and BDNF.

Keywords: neurotrophic factors, IL-1, cytokines

Integration and differentiation of human embryonic stem cells transplanted to the chick embryo Goldstein R.S. ${ }^{1}$, Drukker M. ${ }^{2}$, Reubinoff B.E. ${ }^{3}$ and Benvenisty

'Gonda Research Center, Faculty of Life Sciences, Bar-Ilan University, Ramat-Gan $52900 ;{ }^{2}$ Dept. of Genetics, The Institute of Life Sciences, The Hebrew University, Jerusalem 91904: ${ }^{3}$ Dept. of Obstetrics and Gynecology, Hadassah University Hospital, Jerusalem 91120

Human embryonic stem (ES) cells are pluripotent cells that can differentiate into a large array of cell types and thus hold promise for advancing our understanding of human embryology and for contributing to transplantation medicine In this study differentiation of human ES cells was examined in vivo by transplantation to early organogenesis-stage chick embryos. Human ES cells were grafted into or in place of epithelial-stage somites of chick embryos of 1.5 to 2 days of development. The grafted human ES cells survived in the chick host, and were identified either by a fixable vital dye or by using a green fluorescent protein (GFP) expressing clone Histological analysis showed that human ES cells are distinguished from host cells by their larger, more intensely staining nuclei. Some grafted cells differentiated en masse into epithelia, while others migrated and mingled with host tissues, including the dorsal root ganglion. Colonies grafted directly adjacent to the host neural tube produced primarily structures with the morphology and molecular characteristics of neural rosettes. These structures contain differentiated neurons as shown by $\beta-3-$ tubulin and neurofilament expression in axons and cell bodies. Axons derived from the grafted cells penetrate the host nervous system, and host axons enter the structures derived from the graft. These show that human ES cells transplanted in ovo survive, divide, differentiate and integrate with host tissues, and that the host embryonic environment can modulate their differentiation. The chick embryo may therefore serve as an accessible and unique experimental system for the study of in vivo development of human ES cells. Keywords: human embryonic stem cells, xenografts, organogenesis, neural differentiation
Immunomodulation of PrP 106-126

Goren O. Hanan E. and Solomon B.

Dept. of Molecular Microbiology and Biotechnology, Tel-Aviv University, Tel-Aviv 69978

Prion diseases are transmissible neurodegenerative pathologies characterized by the accumulation in the brain of altered forms of the normal cellular prion protein (PrPc), termed PrP scrapie (PrPsc). Previous studies have shown that a synthetic peptide homologous to residues 106-126 of $\operatorname{PrP}(\operatorname{PrP} 106-126)$ maintains many characteristics of PrPsc. Like PrP, peptide 106-126 has the ability to adopt a beta-sheet conformation and to form amyloid fibrils and induce apoptosis in neurons. Moreover, the neurotoxicity of the peptide requires the expression of endogenous $\operatorname{PrP}$, and the peptide induces hypertrophy and proliferation of astrocytes and activation of microglial cells in-vitro. These data suggest that the region including residues 106-126 might be an essential site in the conversion of PrPc to PrPsc.

Antibodies are known to act as chaperons and are able to stabilize protein structure and/or induce conformational changes. Thus, site directed antibodies might interfere with aggregation processes and/or inhibit prion replication. In order to investigate antibodies ability to interfere with aggregation processes we used active and passive immunization approaches. Polyclonal sera generated by administration of filamentous phage displaying peptide 106-126 as the antigen of choice, was shown to prevent 106-126 peptide aggregation as well as dissolve already formed fibrils and protect PC 12 cells from death induced by 106-126 fibrils. Two monoclonal antibodies generated using $\mathrm{KLH}$ as the protein carrier were shown to share the sera characteristics towards 106-126 peptide and where further analyzed for their ability to induce those changes with the whole protein

Keywords: prion protein, phage display, antibodies, chaperons

Effect of antidepressants on abnormalities in circadian rhythm and feedback regulation of the hypothalamic-pituitary adrenal (HPA) axis in prenatally-stressed rats

Gorodetsky E. ${ }^{1}$, Bejar C. ${ }^{1}$, Poltyrev T. ${ }^{1}$, Youdim MBH. ${ }^{2}$ and Weinstock M.

IDept. of Pharmacology School of Pharmacy. Hebrew University, Jerusalem and ${ }^{2}$ Eve Topf and US National Parkinson's Foundation Centers for Neurodegenerative

Diseases, Technion, Haifa.

Prenatal stress impairs the feedback regulation of HPA axis and capability of the organism to cope in stressful situations (Weinstock, Prog. Neurobiol. 65: 427-451 [2001]). This study compared the circadian rhythm for corticosterone (COR) in adult control [C] and prenatally-stressed [PS] rats, with and without treatment with a tricyclic antidepressant, amitriptyline or a novel brain-selective MAO inhibitor, TV-3326. The female offspring of rats stressed by daily restraint during the last week of pregnancy were housed in a 0700-1900 hr light cycle and given water, amitriptvline $(4.5 \mathrm{mg} / \mathrm{kg} / \mathrm{dav})$ or TV-3326 (17 mg/kg/day) from age of $6-13$ weeks in the drinking water to avoid the stress of injection. When aged 16 weeks, blood $(50 \mu \mathrm{l})$ was taken from the tail at 0800,1200 1500 and $1800 \mathrm{hrs}$ for determination of circadian rhvthm of plasma COR. In other rats, blood was taken before (at 0800-0900) and $30 \& 90 \mathrm{~min}$ after exposure a novel environment. Plasma COR peaked earlier in PS (at $1500 \mathrm{hr}$ ) than in $\mathrm{C}$ rats $(1800 \mathrm{hr})$. In both groups, novelty stress increased plasma COR 2.5 -fold at $30 \mathrm{~min}$. This declined significantly by $90 \mathrm{~min}$ in C but not in PS rats. Neither antidepressant normalized the circadian rhithm in PS rats, but selectively reduced the plasma COR at $90 \mathrm{~min}$ in PS rats. In conclusion, antidepressants appear to be able to correct the abnormality in feedback regulation of the HPA axis in PS rats in keeping with their antianxiety effect, but do not affect the control of its circadian rhythm by the suprachiasmatic nucleus. Keywords: antidepressant, circadian rhythm, corticosterone, novelty stress

The role of interleukin-1 in HPA axis regulation following stress or adrenalectomy

Goshen I. ${ }^{1}$, Weidenfeld $\mathrm{J}^{2}$ and Yirmiva R.

${ }^{T}$ Dept of Psychology. The Hebrew University of Jerusalem. Jerusalem: ${ }^{2}$ Dept. of Neurology. Hadassah University Hospital, Jerusalem

Interleukin-1 (IL-1) is a pleiotropic crtokine, which is known to activate the hypothalamo-pituitary-adrenal (HPA) axis Recent studies demonstrate induction of brain IL-1 following stress. To further examine the role of IL- 1 in stress-induced regulation of the HPA axis, we used mice with deletion of IL-I receptor type I (IL-1rKO). When exposed to mild 
psychological (4-minutes loud noise) or metabolic (7.5mg/mouse 2-deoxyglucose) stressors, IL-1rKO mice displayed significantly lower increase in corticosterone secretion, compared to wild-type (WT) controls. However, when exposed to stronger stressors $(60$-minutes restraint or $15 \mathrm{mg} / \mathrm{mouse} 2$-deoxyglucose), IL-1rKO and WT controls showed a similar increase in corticosterone secretion. These results suggest that IL-1 plays an important role in the regulation of the HPA axis response to mild psychological and metabolic stressors. However, stronger stressors may induce other mediators that can activate the HPA axis in the absence of IL-1. Brain IL-1 was shown, by us and others, to be increased following adrenalectomy (ADX), suggesting a role for IL-1 in the regulation of the HPA axis feedback. To examine this hypothesis, mice were adrenalectomised, and post operational serum ACTH levels were measured. Five days following ADX, both IL-1rKO and WT mice demonstrated a mild increase in ACTH levels compared to their sham-operated controls. Eight days following ADX, ACTH levels in IL-1rKO remained mildly increased, while WT mice displayed an 80-fold increase in ACTH compared to sham-operated mice. These results suggest that the increase in IL-1 levels following stress and adrenalectomy plays a critical role in HPA axis regulation under these conditions.

Keywords: interleukin-1 (IL-1), stress, corticosterone, ACTH adrenalectomy

Local injection of bone-marrow derived dendritic cells pulsed with specific myelin-related antigens stimulates functional recovery from spinal cord injury Gothilf A. ${ }^{1}$, Hauben E. ${ }^{1}$, Cohen A. ${ }^{2}$, Schwartz M. ${ }^{1}$ ${ }^{T}$ Dept. of Neurobiology, Weizmann Institute of Science, Rehovot ${ }^{2}$ Proneuron Ltd., Industrial Park Ness-Ziona

The outcome of spinal cord injury is more devastating than might be expected from the initial insult. This is due to a selfpropagating process of secondary degeneration of neurons that escaped the primary insult. Our group has demonstrated that an inadequate immune response in the CNS is in part responsible for the continuation of this harmful process. It was recently shown by our laboratory that by boosting the immune response in a specific and controlled manner it is possible to protect spared neurons within the CNS from this process of degeneration. Both passive transfer of $\mathrm{T}$ Lymphocytes reactive to CNS autoantigens or immunization of the animal with such CNS specific antigens was shown to lead to a significantly better outcome of trauma to the CNS (Moalem et al, Nat Med 5:49-55 [1999]; Hauben et al, J of Neuroscience 20:6421-6430 [2000]).

In this work we propose that a similar effect can be achieved by manipulating the post traumatic immune response with the use of bone marrow derived dendritic cells pulsed with myelin peptides or with altered myelin peptides (A91) that lack the risk of causing experimental autoimmune encephalitis (EAE). We have found in a model of contusive spinal cord injury in rats that local administration of dendritic cells prepulsed with myelin basic protein (MBP) or with A91, directly into the site of injury, significantly improves recovery as measured by functional outcome of hind limb movement and confirmed by morphological criteria. Dendritic cells injected into the site of injury as far as two weeks after the injury had a beneficial effect on recovery as well. Nonpulsed dendritic cells or those pulsed with a nonrelevant antigen had no effect on recovery. Other possible routs of administration of dendritic cells to the injured animal were also examined. The fact that a positive effect of the dendritic treatment could not be achieved in injured thymectomized rats, which are deprived of mature $T$ cells, suggests that the mechanism whereby dendritic cells display their effect is $\mathrm{T}$ cell dependant, similar to that of vaccination involving antigen specific activation of the immune system. Vaccination with dendritic cells may be an effective way of achieving beneficial immunomodulation following spinal cord injury.

Keywords: CNS trauma, dendritic cells, autoimmunity, neuroprotection

Modulation of prepulse inhibition of the startle reflex (PPI) induced by prenatal injection of an anti-mitotic agent in the rat

Gourevitch R. ${ }^{1}$, Le Pen G. ${ }^{1}$, Canal N.M. ${ }^{2}$, Wanstok N. ${ }^{1}$,

Sandner G. ${ }^{2}$, Jay T.M. ${ }^{3}$ and Krebs M.O.'

${ }_{I}$ INSERM-EMI OI 17-Paris V, Hop Sainte-Anne, Paris, France; ${ }^{2}$ INSERM U405, Faculte de médecine, Strasbourg, France ${ }^{3}$ NAMC, CNRS UMR 8620, Univ. Paris-sud, Orsay, France

Prepulse Inhibition (PPI) of the acoustic startle response is the normal reduction of the startle response to a stimulus when this is preceded by a weaker, non-startling stimulus. PPI deficits are often associated with schizophrenia, a disorder that could be at least in part the consequence of neurodevelopmental abnormalities, especially in the hippocampus and its connections.

Pregnant female Sprague-Dawley rats were IP injected with either an anti-mitotic agent (methylazoxymethanol, MAM, 25 $\mathrm{mg} / \mathrm{kg}$ ) or with saline on gestational day 17 , a critical period for hippocampal development. Their offspring were tested when turned adult ( 4 months) in a PPI paradigm using different prepulse duration (PD) conditions (Canal \& al. Psychopharmacology 156: 169-176 [2001]).

MAM animals exhibited similar baseline startle reflex as controls, but displayed non significant decreased PPI in classical conditions (prepulse-pulse interval $80 \mathrm{~ms}$; PD $20 \mathrm{~ms}$ ). When prepulse-pulse interval was set at $20 \mathrm{~ms}$, the difference between treated and control rats appeared to depend on PD MAM animals had significantly impaired PPI responses when PD was 160 but not 5 or $20 \mathrm{~ms}$; there was a tendency for impairment in 80 and $1280 \mathrm{~ms}$ PD conditions. In both groups, the maximum inhibition was observed in the $20 \mathrm{~ms} P D$ condition. There was no overall treatment effect, but a significant interaction between treatment and parametric conditions, suggesting that prepulse temporal conditions have a specific influence in MAM rats.

This narrow "temporal window" for PPI efficiency in MAM treated animals might be somewhat comparable to the PPI modulation seen with dopaminergic and serotoninergic agonists. These findings have to be discussed in the light of developmental and pharmacological theories of schizophrenia. Keywords: prepulse inhibition; temporal parameter;

methylazoxymethanol; schizophrenia; neuro development

Activity-dependent neuroprotective protein (ADNP): a regulator of rodent brain development and neuronal

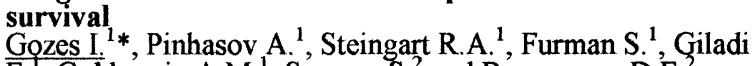

E. Goldsweig A.M. ${ }^{1}$, Servoss S. ${ }^{2}$ and Brenneman D.E.

Clinical Biochemistry, Sackler School of Medicine, Tel Aviv

University; ' $S D M P, L D N, N I H$, Bethesda, MD, USA.;

*igozes@post.tau.ac.il

Activity-dependent neuroprotective protein (ADNP) is a vasoactive intestinal peptide (VIP) responsive gene (Bassan et al J.Neurochem.72:1283-1293 [1999]; Zamostiano et al, J Biol. Chem. 276: $708-714$ [2001]). The current study assessed the role of ADNP in VIP-associated functions, embryonic development and neuroprotection. A first step evaluated ADNP expression during development. Results showed that the embryonic mouse ADNP gene was expressed during early gestation: detected at E7.5 and increased on E9.5. Expression was augmented in the brain (E12) and was sustained throughout embryogenesis. A second step measured ADNP subcellular localization and potential processing in glial cells Results showed intact ADNP-like immunoreactivity $(\sim 120 \mathrm{kD})$ in the rat astrocytic nucleus, the cytoplasm and the extracellular milieu and increased ADNP content following VIP treatment. Lower molecular weight ADNP-immunoreactive bands were also observed. Subcloning of ADNP into a vector containing the Herpes VP22 protein, that is able to penetrate through cell membranes, and assessments of VP22-ADNP neuroprotective activities indicated protection of rat pheochromocytoma cells against oxidative stress. Finally, establishment of ADNP gene knockout mice indicated that homozygous ADNP-knockouts die in the uterus. Macroscopic analysis revealed cranial neural tube closure failure and death on E8.5-9.0. Incubation of E8.5 mouse embryos with VIP was shown to result in robust embryonic growth (Gressens et al, Nature 362,155-158, 1993). Results now indicate increased ADNP gene expression in the presence of VIP. Thus, ADNP may mediate the VIP-stimulated embryonic growth and neuroprotection while serving a vital role in brain maturation.

Support: BSF, Neufeld, ISF, Gildor, ISOA, NIH we thank Dr. Heiner Westphal.

Keywords: VIP, ADNP, embryogenesis, neuroprotection

Shaping and protecting the brain: activity-dependent

neuroprotective protein
Gozes I. ${ }^{*}$, Pinhasov A. ${ }^{1}$, Steingart R.A. ${ }^{1}$, Furman S. ${ }^{1}$, Giladi

E. , Goldsweig A.M. ${ }^{1}$, Servoss S. $^{2}$ and Brenneman D.E.

${ }^{I}$ Clinical Biochemistry, Sackler School of Medicine, Tel Aviv University; ${ }^{2}$ SDMP,LDN, NIH, Bethesda, MD, USA.

* igozes(a)post.tau.ac.il

Activity-dependent neuroprotective protein (ADNP), a vasoactive intestinal peptide (VIP) responsive gene (Zamostiano et al, J. Biol. Chem. 276: 708-714 [2001]), contains nine zinc fingers, a proline-rich region, a nuclear bipartite localization signal, a homeobox domain profile and.a 
nuclear export sequence. Current studies show intact ADNP-like immunoreactivity $(\sim 120 \mathrm{kD})$ in the rat astrocytic nucleus, the cytoplasm and the extracellular milieu and increased ADNP content following VIP treatment. Subcloning of ADNP into a vector containing the Herpes VP22 protein that is able to penetrate through cell membranes and assessments of VP22-ADNP activities indicated protection of rat pheochromocytoma cells against oxidative stress. Part of the ADNP neuroprotection may be attributed to decreases in the pro-apoptotic protein $\mathrm{p} 53$. Peptide scanning analysis identified an eight amino acid peptide (NAP) within the ADNP sequence that mimics the ADNP neuroprotection (Leker et al, Stroke. 33: 1085-1092 [2002]). To further assess ADNP's roles in the intact organism, ADNP knockout mice were established. Results showed that homozygous ADNP-knockouts suffer cranial neural tube closure failure and death on E8.5-9.0. Expression of OCT4, a gene associated with germline maintenance, was markedly augmented, while expression of PAX6, a gene crucial for cerebral cortex formation, was abolished in the embryos and the brain primordial tissue of the knockout embryos, respectively. Incubation of E8.5 mouse embryos with VIP was shown before to result in robust embryonic growth and results now indicate increased embryonic ADNP gene expression in the presence of VIP. Thus, the vital gene, ADNP, may mediate the VIP-stimulated embryonic growth and neuroprotection.

Support: BSF, Neufeld, ISF, Gildor, ISOA, NIH we thank Dr. Heiner Westphal.

Keywords: neuropeptides, neuroprotection; homeobox genes; neurodevelopment

Toward the neurostructural theory in drug abuse: evidence from in vivo and in vitro studies

Green T. ${ }^{1}$, Kinor N. ${ }^{1}$, Hyman T. ${ }^{2}$ and Yadid G. ${ }^{1}$

${ }^{7}$ Bar Ilan University, Ramat Gan, Israel and ${ }^{2}$ Laboratory of Cellular Carcinogenesis and Tumor Promotion, National Cancer Institute, National Institutes of Health, Bethesda. Maryland USA

Neurotrophic factors such as glial cell line-derived neurotrophic factor (GDNF) have been implicated in many forms of plasticity within the brain, including drug addiction. Previously we described that activation of the dopamine-1 $\left(D_{1}\right)$ receptor in a human astrocyte cell line, SVG, increases GDNF levels. Therefore, we examined the effect of cocaine on GDNF and $D_{1}$ receptor expression as compared to amphetamine and morphine in SVG cells. Twenty-four hr incubation of SVG cells with cocaine significantly lowered $D_{1}$ receptor and GDNF mRNA levels (detected using Reverse Transcriptase Polymerase Chain Reaction [RT-PCR]) and increased levels of signal transducers and activators of transcription (STAT) (via scanning and analysis of cDNA arrays). Neither amphetamine nor morphine altered these parameters. Furthermore, in vivo studies demonstrate that rats that have been trained to self-administer cocaine have decreased levels of striatal GDNF, confirming and extending the in vitro findings. In conclusion, cocaine in vivo and in vitro has a direct and specific effect on extraneuronal cells, in addition to its known effect on the neuronal dopamine transporter. Decrease in GDNF neurotrophic support may increase local neuronal vulnerability. Keywords: cocaine, astrocytes, GDNF, $D_{1}$ receptor, STATs

Apoptosis and alternative cell deaths in the retinal tissue Guimaraes C.A. ${ }^{1}$, Benchimol M. ${ }^{2}$, Amarante-Mendes $\mathrm{G}^{3}$ and Linden R.

${ }^{I}$ Institute of Biophysicsm - UFRJ, Rio de Janeiro, Brazil: ${ }^{2}$ Univesidade Santa Ursula, Rio de Janeiro, Brazil; ${ }^{3}$ Institute of Immunology - USP, Rio de Janeiro, Brazil

Distinct types of cell death $(\mathrm{CD})$ occur either during development and pathologies. Apoptosis and autophagy share some hallmarks as cell shrinkage and membrane integrity maintenance. Our aim was to identify the types of $C D$ induced by anisomicyn in the retina and their mechanisms of execution. Ultrastructural analysis of explants treated with anisomycin showed of both autophagic and apoptotic features. Bongkrekic acid, a mitochondrial pore inhibitor, completely inhibited CD induced by anisomycin in retinal explants. Ac-LEHD-CHO Z-DEVD-FMK and Ac-VEID-CHO, caspase-9, -3 and -6 inhibitors respectively, partially inhibited CD induced by ANI. Immunohistochemistry for activated caspases-9, activated caspase- 3 and TUNEL-staining stained approximately $50 \%$ of condensed cells in tissue sections. 3-methyl-adenine (3MA), an autophagy inhibitor, reduced $\mathrm{CD}$ induced by anisomycin. Caspase-9 and caspase- 6 inhibitors had synergic effect upon $\mathrm{CD}$ induced by anisomycin. Co-incubation of retinal explants with 3MA and caspase-9 inhibitors did not have synergic effect upon $\mathrm{CD}$ induced by anisomycin and TUNEL, activated caspases-9 and activated caspase-3 staining decreased in explants treated with 3MA. Furthermore co-incubating of explants with caspase- 3 and caspase- 6 inhibitors did not have synergic effect upon $\mathrm{CD}$ induced by anisomycin

These results suggest that anisomycin induces distinct types of $\mathrm{CD}$, all dependent on mitochondrial commitment and caspase activation. The first type of CD involves autophagy, caspase-9 and -3 activation and is TUNEL+, suggesting an overlap between apoptosis and autophagy. The other CD type involves caspase- 6 and is TUNEL-. A third CD pathway is dependent on caspase- 9 activation and occurs under caspases- 3 and -6 inhibition

Keywords: cell death, caspases, autophagy, retina

\section{Orientation selectivity in $\mathrm{V} 1$ of the alert monkey}

Gur M. ${ }^{1,2}$, Kagan I. ${ }^{1}$ and Snodderly DM.

${ }^{1}$ Biomedical Engineering, Technion, Haifa; ${ }^{2}$ Schepens Eye

Research Institute, Harvard Medical School

Area V1 is unusual among all primate cortical areas in its neural cell density, cortical thickness, and intricate histology. Physiological recordings, however, emphasize overal integrating features such as ocular dominance and orientation selectivity. We have previously shown that in the alert monkey physiological properties of single cells reflect alternating anatomical layers structure. Here we study the relation of single cells orientation selectivity to the cells spontaneous activity and receptive field (RF) size, and how these properties are related to the anatomical location of the cell. Single cells were recorded from area V1 of an alert monkey performing a simple fixating task. The cells spatial organization was studied by drifting increment and decrement bars and by sinusoidal gratings. Orientation selectivity was defined by the orientation tuning curve bandwidth and circular variance. Orientation selectivity was clearly correlated with RF size and spontaneous activity. These 3 measures were strongly predicted by the layer of origin such that small RFs, low spontaneous activity, and a high degree of orientation selectivity were found in the output layers $2 / 3,4 \mathrm{~B}$ and 5 while the reverse was true for the input layers $4 \mathrm{~A}, 4 \mathrm{C}$ and 6 . We conclude that the conjunction of these physiological measures and their anatomical characterization reflect interactions between excitatory and inhibitory mechanisms. When excitation is strong, large RFs, high spontaneous activity and a low degree of orientation tuning are found while when significant inhibition is present, RFs shrink, spontaneous activity almost disappears and orientation selectivity is high.

Keywords: visual cortex, orientation selectivity

\section{Assesment of vasomotor reactivity for prediction of syncope in patients with orthostatic hypotension. Gurevich T. Yu, Gur A. Yu, Korczyn A.D., Giladi N. and Bornstein N \\ Neurology Dept., Tel-Aviv Sourasky Medical Center.}

Background: Orthostatic hypotension $(\mathrm{OH})$ is a common neurological symptom. It is not clear why some patients can tolerate $\mathrm{OH}$ while others faint. Syncope is a result of severe cerebral hypoperfusion and cerebral autoregulation failure.

Objective: To assess cerebral autoregulation in patients with $\mathrm{OH}$ and history of syncope.

Methods: 29 patients with $\mathrm{OH}$ age $72.9 \pm 9.8$ years, mean orthostatic decrease of systolic blood pressure of $33.7 \pm 129$ $\mathrm{mmHg}$ after three minutes of standing, were assessed for VMR of middle cerebral arteries (MCA) and vertebral arteries (VA) using TCD (Rimed) after injection of $1 \mathrm{~g}$ acetazolamide i.v. 13 patients had experienced at least 2 documented syncope episodes over the preceding year. Patients with carotid stenosis greater than $75 \%$ were excluded from the study.

The percent difference between blood flow velocities before and after acetazolamide injection was defined as VMR\% and the results were compared by Mann-Whitney test.

Results: Patients with syncope had worse VMR in both RT MCA and RT VA than those without syncope $(12.1 \pm 30.9 \%$ vs $40.6 \pm 19.9 \%$ in RT MCA $(\mathrm{P}<0.005)$ and $21.1 \pm 36.4 \%$ vs $49.2 \pm 57.4 \%$ in RT VA $(P<0.05)$, while there was no significant difference in these parameters on the left side. A strong statistical trend of decrease in mean VA VMR\% in patients with syncope was found $(19.9 \pm 38.9 \%$ in patients with syncope vs. $38.3 \pm 32.8 \%$ in patients without syncope; $p=0.09$ )

Conclusions: Impaired VMR may contribute to the development of syncope in patients with $\mathrm{OH}$. The acetazolamide test is useful for predicting the risk of syncope in patients having $\mathrm{OH}$.

Keywords: orthostatic hypotension, syncope, Vasomotor reactivity 
Myosin VI and hereditary hearing loss: from mouse to man and back to mouse

Guy K. ${ }^{1}$, Ahituv N. ${ }^{1}$, Solomonik I. ${ }^{2}$, Gasparini P. ${ }^{3}$ and Avraham K.B. ${ }^{1}$

${ }^{1}$ Dept. of Human Genetics and Molecular Medicine, Sackler School of Medicine, Tel Aviv University, Tel Aviv, Israel;

${ }^{2}$ Faculty of Life Sciences, Bar-Ilan University, Ramat-Gan, Israel; ${ }^{3}$ Genetica Medica, Dip. Patologia Generale, II Universite di Napoli and Tigem, Italy

The past few years have provided an explosion in our understanding of how the inner ear functions. This dramatic increase is due in large part to the genes found to be associated with nonsyndromic hearing loss. Since 1997, 29 genes have been found, and these have provided clues about auditory transduction, ion homeostasis, and inner ear development. In particular, mouse models for human deafness, with mutations in orthologous genes, have revealed essential information about the pathophysiology caused by these mutations. One group of proteins frequently associated with hearing loss are the myosins, three of which were discovered thanks to their corresponding mouse mutants. Myosins are molecular motors that move along actin filaments and have been implicated in various cellular functions such as cell movement, membrane traffic, and signal transduction. An intragenic deletion in myosin VI (MyO6) leads to deafness and vestibular dysfunction in Snell's waltzer mice. A missense mutation in an Italian family is associated with autosomal dominant progressive hearing loss (C442Y) (Melchionda et al, $A J H G$ 69:635-640 [2001]). The following suggests that the deafness in this family is due to the MYO6 missense mutation: the segregation of this mutation with the affected individuals in the family, the previous association of myosin VI with deafness, and the conservation of the mutated residue. We have now confirmed that this mutation is the cause of deafness in humans, since reproduction of this mutation in transgenic mice has revealed a similar progressive hearing loss. A morphological analysis of the transgenic mice demonstrates the pathophysiology of this mutation.

Keywords: sensory system, hearing loss, myosin, transgenic mice

\section{Global-local processing among elderly with and without} insomnia: a comparison with young adults

Hadad B. Shurkin D. and Haimov I.

Dept. of Behavioral Science, Emek Yezreel Academic College Disturbed sleep, an important cause of human misery at any stage of life, exacerbates with advancing age. Complaints of difficulty in initiating and maintaining sleep and daytime drowsiness are more prevalent among the elderly than in any other group. Likewise, aging is associated with a decline in many aspects of visual processing including changes in global-local processing. However, little is known about the interaction between sleep disturbances and visual processing of global and local aspects among elderly people. Thus, we examined whether insomnia is indeed associated with age-related changes in this global-local processing. Results showed that the global and the local structures were equally manifested in young adults, along with a tendency toward a global advantage. Elderly subjects without insomnia, on the other hand, showed a reversed tendency of faster response time for the local aspects, indicating the processing dominance of the local level of visual stimuli in elderly. Furthermore, elderly subjects with insomnia showed a significant advantage for the local structure and an asymmetric local-to-global interference, suggesting that the ability to integrate individual elements into a coherent pattern deteriorates with age, and becomes substantially impaired among elderly insomniacs. Overall, the findings imply that, at least in some aspects, sleep disturbances may account for perceptual and cognitive decline in the elderly. Keywords: aging, global-local processing, visual perception, insomnia

The wasp Ampulex Compressa injects venom directly into prey central nervous system

Haspel G., Rosenberg L.A. and Libersat F. Zlotowski Center for Neuroscience and Dept. of Life Sciences, Ben-Gurion University, Beer-Sheva

In contrast to other venomous animals that paralyze their prey, the venomous parasitoid wasp Ampulex Compressa subdues its prey by the induction of a bout of grooming followed by a five-week long lethargic state. During the lethargic state the cockroach is docile enough for the wasp to pull it into a burrow where the wasp larva eats it alive. The cockroach is always stung by the wasp twice, first in the thorax and then in the head.
To localize the site of stinging we injected wasps with a mixture of $\mathrm{Cl} 14$ radiolabeled amino acids. After the amino acids were incorporated into the venom, we allowed the wasps to freely sting several cockroaches

First, the amount of radioactivity in the ganglia of stung cockroaches was assessed using liquid scintillation of the different ganglia and tissue. Significantly higher levels of radioactivity were detected in the head ganglia and the first thoracic ganglion. In contrast, radioactivity levels in the third thoracic ganglion and non-neuronal tissue were much lower and comparable to control values.

Second, microscopic emulsion autoradiography was carried out to determine the precise location of injection. Radioactivity was detected in the central area of the brain ganglion, the subesophageal ganglion and the first thoracic ganglion. No radioactivity could be detected in the second thoracic ganglion or in ganglia stung by non-radiolabeled wasps.

To our knowledge, this is the first direct evidence of a venomous animal stinging into the central nervous system of its prey.

Keywords: venom, wasp, cockroach, central-body

Mirror-symmetry organization of human occipito-temporal object areas

Hasson U. ${ }^{1}$, Harel M. ${ }^{1}$, Levy I. ${ }^{1,2}$ and Malach R. ${ }^{1}$

Dept. of Neurobiology, Weizmann Institute of Science, Rehovot 76100: ${ }^{2}$ The Interdisciplinary Center for Neural Computation, Hebrew University of Jerusalem, Jerusalem 91904

Object images activate a large and complex array of high order visual areas in the human cortex, ranging from dorsal occipito-parietal cortex to ventral occipito-temporal cortex. To search for consistent patterns within this array we performed a detailed analysis of fMRI activation in 12 experienced subjects along four basic dimensions (object selectivity, eccentricity, motion selectivity and visual meridians). Our results revea seven consistently distinct category-related entities situated in the cortex adjoining early visual areas. These include: two face-related regions, three object-related regions and two building-related regions. Interestingly, we found that the complex pattern is organized in a dorso-ventral, large-scale object-based mirror symmetry. Furthermore, correlating this pattern to the map of visual field eccentricity, we find that the entire network of object areas can be related to a single eccentricity map. We hypothesize that this large-scale organization points to a possible development of high order object areas through extension and specialization of a single proto-representation.

Funded by Israel Academy 8009 and 644/99 grants.

Keywords: visual cortex, fMRI, object recognition, topography

\section{Lack of paternal care reduces spine density in the limbic} cortex

Helmeke C., Ovtscharoff jun. W. and Braun K.

Dept. Zoology/Developmental Neurobiology, Otto von Guericke University, 39008 Magdebuirg, Germany.

Email: katharina.braun@nat.uni-magdeburg.de

Previous studies in trumpet-tailed rat Octodon degus have demonstrated that, quite analogous to the functional maturation of sensory and motor cortices, which strongly depend on sensory or motor stimulation, the development of synaptic connections in limbic brain regions are modulated by early postnatal emotional experiences. Periodic and chronic interruptions of parental care during the first weeks of life induce a significant increase of dendritic spines in the limbic dorsal anterior cingulate cortex (ACd) (Helmeke C, Ovtscharoff jr et al, 2001, Cerebral Cortex, 11: 717). In the present study the contribution of paternal care on the development of synaptic composition in the $\mathrm{ACd}$ was analyzed light and electron microscopically. The comparison of pups which were either raised with or without their father revealed in the fatherless animals a $20 \%$, decreased spine density on apical and basal dendrites of layer II/III pyramidal neurons in the ACd.

Such experience-induced modulations of synaptic inputs in higher associative and limbic cortical areas appear to shape the limbic synaptic networks and thereby may determine cognitive and psychosocial capacities during early and later stages of life. Supported by SFB 426.

Keywords: limbic system, development, synaptic plasticity, dendritic spines 
Assessment of tissue functionality in vicinity of brain lesions using DTI and fMRI

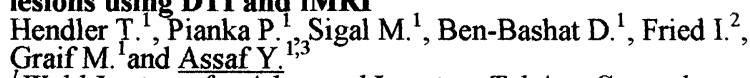

${ }^{I}$ Wohl Institute for Advanced Imaging, Tel Aviv Sourasky Medical Center, Tel-Aviv; ${ }^{2}$ Functional Neurosurgery Unit, Tel Aviv Sourasky Medical Center, Tel-Aviv; ${ }^{3}$ School of Chemistry, Tel Aviv University, Tel-Aviv 69978

In the last decade functional MRI (fMRI) gradually evolved to become a clinical tool for pre-surgical evaluation of critical functionality in vicinity of brain lesions. fMRI signals relate to activated gray matter only. However the connectivity of white matter is crucial for functionality of a region and should not be damaged during surgery. Diffusion tensor imaging (DTI) is a well-established method for in-vivo mapping of the white matter directionality and organization, but its clinical application has not been fully explored. Our aim in this study was to explore the added value of the combined methodologies in brain surgery. DTI is based on the anisotropic motion of water molecules in white matter. While the diffusion of water parallel to long the long axis of the neuronal fibers is free, perpendicular to the fibers it is disturbed. Recently, the concept of three-dimensional fiber tracking based on DTI data was introduced. With this methodology it is possible to produces fiber tracts that corresponds with large fiber bundles such as the pyramidal tract, corpus callosum, optic radiation, corona radiata and thalamic radiation. In relation to brain tumors, one can think of three ways brain lesion and tumors can affect white matter fibers: cutting, pressing or infiltrating. Indeed, three-dimensional fiber tracking helped to visualize critical white matter bundles and their relation to the tumor. White matter bundles that were cut, pressed or infiltrated by the lesion could be identified and relation of these bundles to task related gray matter activation could be visualized.

Keywords: MRI, diffusion, DTI, fMRI, white matter

Glial cell coupling and growth in sensory ganglia after axotomy: ultrastructural evidence Huang T. Y ${ }^{1}$, Hanani M. ${ }^{1}$, Cherkas P.S. ${ }^{1}$, Ledda M. ${ }^{2}$ and Pannese E.

${ }^{1}$ Laboratory of Experimental Surgery, Hadassah University Hospital, Mount Scopus, Jerusalem 91240; ${ }^{2}$ Institute of Histology, Embryology and Neurocytology, University of Milan, 14 Via Mangiagalli, I-20133 Milan, Italy

Axotomy induces profound changes in primary sensory neurons and often produces neuropathic pain states, but very little is known about its effects on satellite glial cells (SCs) in dorsal root ganglia (DRG). We have shown previously that dye coupling among SCs was increased after axotomy. In this research we studied the ultrastructural basis of this change by correlating intracellular dye injections and serial section electron microscopy (EM). Lucifer yellow injections showed that in control ganglia $76.2 \%$ injected SCs were not coupled to other SCs, and $20.7 \%$ were coupled within a glial envelope around a given neuron, and very few $(3.1 \%)$ were coupled between glial envelopes around different neurons. However, in axotomized ganglia the incidence of coupling between glial envelopes increased by 7 -fold $(p<0.0001)$ and those of coupling within an envelope increased to $30.5 \%(p<0.001)$. Some glial processes from glial sheath extended into adjacent connective tissue. Neurons were not coupled to other cells in control or axotomized ganglia. Serial section EM showed that after axotomy SCs extended new processes into surrounding connective tissue and formed new bridge-like connections between glial envelopes belonging to different neurons. Such bridge-like connections were absent in control ganglia. The number of gap junctions between SCs increased 6.5-fold $(p<0.01)$ after axotomy. Thus, glial dye coupling is apparently mediated by gap junctions. We propose that axotomy induces growth of perineuronal SC sheaths, leading to new contacts between SCs enveloping different neurons and to formation of new gap junctions between SCs. These changes may be an important mode of glial plasticity and contribute to neuropathic pain.

Keywords: axotomy, dorsal root ganglia, satellite glial cells, gap junctions, dye coupling, ultrastructure

\section{Receptors for neurotransmitters on $\mathrm{T}$ lymphocytes as} regulators of immune cells Ilani T. ${ }^{1}$, Strous RD. ${ }^{2}$ and Fuchs $S .^{1}$ ${ }^{T}$ Dept. of Immunology, Weizmann Institute of Science, Rehovot 76100; ${ }^{2}$ Beer-Yaakov Mental Health Center.

In recent years it became apparent that peripheral blood lymphocytes express receptors for various neurotransmitters (NTs). Their existence on peripheral cells is unclear, as NTs are generally confined to the CNS and unavailable for peripheral binding. It has been suggested that neurotransmitter receptors are activated by peripheral innervations, yet their role remains unresolved. We show that NT receptors play a role in a surveillance pathway of the brain on immune cells. To study this pathway, we chose one neurotransmitter, dopamine, and focused on one of its receptors, the $D_{3}$ dopamine receptor $\left(D_{3} R\right)$, which is expressed on $T$ cells. $D_{3} R$ is known to be involved in neuropsychiatric disorders and serves as a target for therapeutic treatment. We demonstrate that $D_{3} R$ on $T$ cells is functionally active, and its activation affects immunological properties of the cells. Interestingly, the immunological changes observed, are confined to only a subclass of highly activated $T$ cells, blasts. Unlike non-activated $T$ cells, blasts can cross the BBB and bind NTs due to high expression of adhesion molecules. In addition, they also express higher levels of $D_{3} R$ than T cells. Dopaminergic activation of blasts changes their cytokine secretion profile and the expression of severa surface markers. Since these effects do not occur in non-activated $T$ cells it seems that the underlying mechanism is meant to activate only those cells that can cross the BBB

Finally, we show that peripheral $T$ lymphocytes of schizophrenic patients behave like blasts in several features. They exhibit higher levels of both $D_{3} R$ mRNA and protein These findings suggest that a central disorder like schizophrenia leads to changes in peripheral immune cells demonstrating a control pathway of the brain on immune cells. Keywords: $\mathrm{D}_{3}$ dopamine receptor, peripheral blood lymphocytes

\section{Involvement of plasminogen activator system in central} nervous system inflammation and demyęlination Irony-Tur-Sinai $\mathrm{M}^{1}{ }^{1}$, Pinto $\mathrm{F}^{1}$, Higazi $\mathrm{AE}^{2}$ and Brenner $\mathrm{T}$. Depts. of Neurology $y^{I}$ and Biochemistry ${ }^{2}$, Hadassah Hebrew University Medical Center, Jerusalem

Extracellular proteolytic enzymes have been implicated in the pathogenesis of demyelinating neuroinflammatory disorders, such as multiple sclerosis (MS) and experimental autoimmune encephalomyelitis (EAE). During EAE, there is concurrent induction of plasminogen activator (PA) and matrix metalloproteinases (MMPs) systems, supporting the concept that neuroinflammatory damage in EAE involves an altered balance between extracellular proteases and their inhibitors. In our study, we tested the involvement of the PA system in EAE, using mice genetically deficient in urokinase PA (UPA) or UPA receptor (UPAR). In comparison to the wild type (wt) UPA knockout (UPA-/-) mice were more severely ill (UPA-/$2.1+0.5$, wt: $0.9+0.3)$ and with longer duration $(23+3$ days versus $12+3$ days). Similarly, UPAR knockout (UPAR-/-) mice also exhibited the disease more severely, which was accompanied with a lack of spontaneous recovery. The death percentage in the UPAR-/- group was $50 \%$ whereas in the wt it was only $7.7 \%$. Addition of octapeptide (A6) (that blocks interaction between UPA and UPAR), to the T-cell proliferation assay in vitro, resulted in a marked inhibition of T-cell reactivity (both in T-cells derived from UPA- $/$ - and wt animals) Our results imply that a network of functionally redundant proteases is involved in EAE and MS progression and recovery. Modulation of the PA system can serve as a potential target for treatment of CNS inflammatory and demyelinating diseases.

Keywords: urokinase plasminogen activator, urokinase plasminogen activator receptor, CNS inflammation, experimental autoimmune encephalomyelitis

Inhibition of beta secretase cleavage of APP (Amyloid Precursor Protein) by active immunization approach Jacoby I and Solomon B.

Dept. of Microbiology and Biotechnology, Faculty of Life Science, Tel Aviv University.

APP (amyloid precursor protein) is subject to post-translational modifications, glycosylation, sulphation and phosphorylation along the intracellular protein secretory pathway. Cleavage of APP occurs in two different ways:

1) The non-amylodogenic pathway in which alpha secretase cleavage $g$ generates a soluble $\mathrm{N}$-terminal APP fragment, which leaves the $C$ terminus attached to the membrane.

2) The amyloidogenic pathway, which generates $A \beta P$ through beta and gamma secretase cleavages involving internalization of APP from the cell surface and its cleavage in late secretory pathways. Recently the beta site cleavage enzyme (BACE) was reported (Vassar et al, Science 286: 735-741 [1999]) and is now subjected for intense research aimed for blocking the enzyme activity.

We are developing a new approach for blocking beta secretase activity, based on immunization with a small peptide representing the cleavage site of beta secretase. Antibodies 
raised recognize the cleavage site of APP and will hopefully interfere with AP formation. Limiting of beta amyloid production by this approach may become an important therapeutic target in Alzheimer disease (AD).

Keywords: Alzheimer's disease, beta amyloid, BACE, inhibition, vaccination

The relationship between the cell cycle and BMP4 signaling in the control of neural crest delamination

Kalcheim C. and Burstyn-Cohen T.

Dept. of Anatomy and Cell Biology. Hebrew University of Jerusalem-Hadassah Medical School. Jerusalem 91120

Delamination of premigratory neural crest cells from the dorsal neural tube depends upon a gradient of BMP4 activity along the neuroaxis, which is generated by changing levels of noggin. The latter are in turn modulated by an inhibitory activity from the dorsomedial somite which coordinates the timing of cell emigration with the elaboration of a migratory mesodermal substrate. Cell-intrinsic mechanisms also regulate delamination. Here we show that neural crest cells synchronously emigrate from the neural tube in the S-phase of the cell cycle. Specific inhibition of the transition from G1 to $S$ both in vivo and in vitro blocks delamination, whereas inhibition at $\mathrm{S}$ or $\mathrm{G} 2$ phases has no immediate effect. Thus, the transition between Gl to S is necessary for the epithelial-to-mesenchymal conversion of crest precursors and may be required for the cells to respond to environmental signals that trigger delamination. The notion is being examined that BMP signaling and the cell cycle features involved in cell delamination are hierarchically linked.

Keywords: neural cest, control of proliferation, migration,

morphogenesis

\section{Bi-phasic modulation by hydrogen peroxide of synaptic} plasticity

Kamsler A. and Segal M

Dept. of Neurobiology, Weizmann Institute of Science, Rehovot 76100

Unlike the proposed role of reactive oxygen species (ROS) in neurodegeneration, acute effects of reactive oxygen on synaptic plasticity are poorly understood. Using rat hippocampal slices we found that exposure to high concentration $(0.5-5 \mathrm{mM})$ of $\mathrm{H}_{2} \mathrm{O}_{2}$ reduces EPSP's in both potentiated and non-potentiated synapses. Exposure of the slices to $20 \mu \mathrm{M} \mathrm{H}_{2} \mathrm{O}_{2}$ did not affect expression of pre-established long term potentiation (LTP) but prevented induction of new LTP, and enhanced long term depression (LTD). Surprisingly, $1 \mu \mathrm{M} \mathrm{H}_{2} \mathrm{O}_{2}$ caused a two-fold increase in LTP compared to controls, and it further enhanced NMDA-independent LTP. Low concentration of $\mathrm{H}_{2} \mathrm{O}_{2}$ also suppressed LTD. Nifedipine, an L-type calcium channel blocker did not affect control LTP but blocked effects of both $1 \mu \mathrm{M}$ and $20 \mu \mathrm{M} \mathrm{H}_{2} \mathrm{O}_{2}$. Calcineurin inhibitors (FK506, Cyclosporin A, but not Rapamycin) acted similarly, and also restored LTP in the presence of $20 \mu \mathrm{M} \mathrm{H}_{2} \mathrm{O}_{2}$. These results suggest that $\mathrm{H}_{2} \mathrm{O}_{2}$ alters NMDA-independent, voltage-gated-calcium channel- mediated LTP by activating calcineurin

Keywords: $\mathrm{H}_{2} \mathrm{O}_{2}$, LTP, calcineurin

\section{Myeloid dendritic cells are activated in secondary} progressive multiple sclerosis: increased CD80 expression, cytokine production and a proinflammatory polarization effect on naïve $T$ cells

Karni A ${ }^{1,2}$, Abraham M. ${ }^{1,2}$, Cai G. ${ }^{1,2}$, Freeman $F^{2,3}$, Hafler D.1,2 Khoury S.J.1,2 and Weiner H.L.',

${ }^{\prime}$ Brigham \& Women's Hospital, ${ }^{2}$ Harvard Medical School, ${ }^{3}$ Dana Farber Cancer Institute

Objective: To study the activation status and function of myeloid dendritic cells in MS.

Methods: Expression of HLA-DR, CD80, CD86, CD40, CD83 and PDL- 1 on and IL-12 \& TNF- $\alpha$ production in myeloid DCs was studied by flow cytometry. The polarizing effect of MS DCs on naïve T-cells was studied using mixed-lymphocyte reaction (MLR) of DC with naive $T$ cells, T-cell stimulation with anti-CD3/CD28 and measurement of IL-2, IL-4, IL-10, IFN- $\gamma$ \& TNF- $\alpha$ in the supernatant by ELISA

Results: Compared to relapsing remitting (RR)-MS, secondary-progressive (SP)-MS showed: $\{1\}$ More DCs expressing CD80 $(19.1 \%$ vs. $7.4 \%, p=0.03),\{2\}$ Higher IL-12 $(4.3 \%$ vs. $1.25 \%, p=0.012)$ and TNF- $\alpha$ production $(9.18 \%$ vs. $3.05, \mathrm{p}=0.042)$ in unstimulated DCs, $\{3\}$ Decreased expression of PDL- $1(3.4 \%, 9.3 \%$ and $12.2 \%$ for SP, RR and HC resp. $\mathrm{p}<0.05)$. DCs incubated with serum from SP-MS $(n=15)$ had higher IL-12 (3.53\%) compared to RR-MS $(1.27 \%, \mathrm{p}=0.009$ $\mathrm{n}=28)$ or $\mathrm{HC}(0.78 \%, \mathrm{p}=0.007, \mathrm{n}=17)$. Post-MLR T-cell stimulation showed T-cells polarized by SP-MS DCs secreted more TNF- $\alpha(225.9 \mathrm{pg} / \mathrm{ml})$ vs. those polarized by HC DCs $(22.7 \mathrm{pg} / \mathrm{ml}, \mathrm{p}=0.049)$. The secreted TNF $-\alpha / \mathrm{IL}-4$ ratio from T-cells polarized by SP-MS DCs was higher than by HC DCs (8.01 vs. $1.45, \mathrm{p}=0.038$ )

Conclusions: Myeloid DCs in SP-MS are activated compared

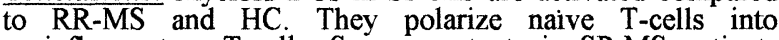
proinflammatory T-cells. Serum contents in SP-MS patients may play a role in DC activation, manifested by its effect on DC IL-12 production. These results demonstrate that the activated DCs may determine the type of immune activity in SP-MS.

Keywords: dendritic cells, multiple sclerosis, co-stimulation, cytokines, $\mathrm{T}$ cells polarization

The expression of mitochondrial complex I subunits, 24-and 51-kDa, is reduced in the frontal and elevated in the parietal cortices of schizophrenic patients

Karry R., Klein E. and Ben Shachar D. Lab. Psychobiology, Dept. of Psychiatry, Rambam Medical center, B. Rappaport faculty of Medicine, Technion

Impairment of the mitochondria, which play a major role in cell function and survival, could provide an explanation for the tremendous heterogeneity of clinical and pathological manifestations in schizophrenia. Previous studies have shown abnormalities in brain mitochondrial morphology as well as in the respiratory chain enzymatic activity in schizophrenia. We have recently reported a disease stage dependent alteration in complex I activity in platelets of schizophrenic patients, which was accompanied by alterations in the expression of the $24 \mathrm{kDa}$ and $51 \mathrm{kDa}$, but not in the $75 \mathrm{kDa}$ subunits of complex I. In the present study we compared the mRNA and protein levels of the three subunits in frontal and parietal post-mortem brain cortices of 14 schizophrenic patients, 15 unipolar patients (UP), 15 bipolar patient (BP) and 15 normal subjects, obtained from the Stanley Foundation Brain Bank. Both mRNA and protein levels of 24- and 51-kDa subunits were significantly reduced in the schizophrenic frontal cortex compared to the controls, while UP patients showed a reduction in $24 \mathrm{kDa}$ protein level, which was less significant than that of the schizophrenic group. No such changes were observed for the BP. In contrast, a significantly increase was observed in mRNA and protein levels of the $24 \mathrm{kDa}$ subunit in the parietal cortex of the schizophrenic patients. Protein level of both subunits was also increased in the BP patients. The $75 \mathrm{kDa}$ subunit did not differ between groups. The present study further demonstrates the malfunctioning of complex I in schizophrenia and supports the relevance of our peripheral findings to the CNS pathology.

Keywords: schizophrenia, mitochondrial complex I, post-mortem brains

\section{Embryo exposure to hypoxic episode affects postnatal}

development ${ }_{\text {Kashtutsky I.4 }}{ }^{1,4}$, Huleihel M. ${ }^{2}$, Hallak M. ${ }^{3}$, Sorokin $Y^{5}$ and Golan $\mathrm{H}$.

Dept. of Developmental and Molecular Genetics ${ }^{l}$. Dept. of Immunology $y^{2}$ and Dept. of Obstetrics and Gynecology, Faculty of Health Sciences and Zlotowski Center for Neuroscience ${ }^{4}$, Ben-Gurion University, Beer-Sheva; ${ }^{5}$ Dept. of Obstetrics and Gynecology, Hutzel Hospital, Detroit,

Michigan, USA

Maternal hypoxia during pregnancy is known as a major risk factor for fetal brain damage. To evaluate the mechanisms of the neuro-developmental damage caused by hypoxia we have studied newborn mice development following 2 hours of exposure of 17 gestation day pregnant mice, to $9 \%$ oxygen and $3 \% \mathrm{CO}_{2}$. Newborn were inspected daily for phenotype, morphogenic parameters, reflex development, muscle strength and coordination. Hypoxia did not affect newborns body weight, however, it increased brain weight of newborns during first postnatal month, as measured at postnatal days 1 (P1), P7, P14 and P21 ( $\mathrm{n}=15-12)$. In addition, hypoxic episode accelerate eyelid opening $(\mathrm{P}<0.001, \mathrm{n}=8-9)$ and teeth eruption, as compared to the control group. No difference was observed in the development of righting reflex between the study groups. Yet, newborns previously exposed to hypoxia delayed in developing the ability to climb on inclining slope and hold themselves to rotating rod. On P8-P9 hypoxia exposed newborn were able to climb on $10^{\circ}-20^{\circ}$ (mean) slope as compared to $40^{\circ}-50^{\circ}$ in the control group $(\mathrm{p}<0.0029,0.013$ $n=6-11,9-10$, respectively). Significant difference was also observed between the groups in the rotarod holding at P10, P11 $(\mathrm{P}=0.006,0.04, \mathrm{n}=6-10,6-4)$. Geotrophism was examined in $50^{\circ}$ slope, a full response was achieved by day 6 in the control group, while only $65 \%$ of the hypoxia exposed newborn responded to the slope on P13. Taken together, hypoxia 
episode at 17 gestation day accelerated the development of some morphogenic parameters and delayed development of muscle strength and orientation of newborns.

Supported by BSF grant contract No. 200172

Keywords: hypoxia, development, brain damage

Separating signal from noise in psychophysics

Katkov M., Tsodyks M. and Sagi D.

Dept. of Neurobiology, Weizmann Institute of Science, Rehovot

Psychophysical discriminations can be viewed as a comparison between two internal responses, each characterized by its mean value and noise amplitude. Performance, measured as percent correct discrimination, is effectively determined by signal to noise ratio. To separate these two response components, available methods impose additional constraints on the decision process or on the stimulus-response transformation. Here we propose a novel approach that only assumes normally distributed one-dimensional internal responses. It is based on minimizing the residual error between measured and modeled psychophysical response levels. This procedure requires a number of stimuli pairs in order to derive an over-complete system of equations for mean and noise response amplitudes. The method was applied to a contrast discrimination task Gabor patches with three contrast levels in two spatial configurations - with flankers and without them - were used as stimuli. Stimuli were presented employing a two alternative force-choice paradigm. Each trial consisted of a sequence of two stimuli having Gabor patches of different contrast levels.

For increasing contrast, results show monotonic increase of mean response values with decreasing slope and monotonic decrease of noise amplitude. The presence of flankers resulted in weaker mean response (inhibitory effect) and higher noise amplitude.

Keywords: contrast discrimination, model, signal-detection theory, lateral interactions

Elongation factor-2 phosphorylation in the rat insular cortex following taste learning

Kats K. ${ }^{1}$, Elkobi A. ${ }^{1}$, Nairn A.C. ${ }^{2}$ and Rosenblum K. Center for Brain and Behavior Research, Haifa University, ${ }^{2}$ Sch Med, Yale University, New Haven, CT, USA

This study was conducted in order to learn whether active regulation of protein synthesis plays a role in memory consolidation. We have examined the possibility that phosphorylation of eEF2 on Thr-56 by eEF2 kinase is correlated with learning processes. Phosphorylation of eEF2 was previously found to be involved in development of the visual cortex (Scheetz et al, Nature Neuroscience 3: 211-216 [2000]). We used novel taste learning paradigms together with immunoblotting analysis of total and phospho-eEF2 levels in the insular cortex in different time points following taste presentation. Phosphorylation of ERKII was used as a positive control. At 2,30,180,360 min and 18 hours following taste presentation ( $n=7$ each group) there was no change in eEF2 phosphorylation following consumption of the same amount of the novel tastant $(0.1 \%$ saccharin) compared with water However, there was a significant increase $(22 \%)$ in eEF2 phosphorylation $20 \mathrm{~min}$ following the offset of novel taste consumption $(\mathrm{p}<0.03$ two-way student $\mathrm{t}$-test, $\mathrm{n}=10)$. This time-dependence of eEF2 phosphorylation in the insular cortex was similar to ERKII phosphorylation: only $20 \mathrm{~min}$ following novel taste presentation there was an increase of $95 \%$ in ERKII phosphorylation $(p<0.02$, two-way student $t$-test $n=10)$. Examination of a sinaptosomal fraction from the insular cortex exhibited a similar increase $(23 \%)$ in eEF2 phosphorylation following exposure to saccharin $(p<0.03$, two-way student t-test $n=10$ ). The results indicate for the first time modulation of eEF2 phosphorylation during learning, and suggest that regulation of pretein synthesis in the relevant cortical area may be correlated with memory consolidation.

Keywords: memory, consolidation, protein synthesis

Antiphospholipid syndrome exacerbates cognitive impairment in an APP mouse model of Alzheimer's disease Katzav A. ${ }^{1}$, Pick C. G ${ }^{3}$, Korczyn A.D. ${ }^{1,2}$, Blank M. ${ }^{4}$, Shoenfeld $\mathrm{Y}^{4}$ and Chapman J.

Depts. of ${ }^{I}$ Physiology \& Pharmacology, ${ }^{2}$ Neurology and ${ }^{3}$ Anatomy, Sackler Faculty of Medicine, Tel Aviv University Tel Aviv $69978{ }^{4}$ Dept of Medicine B and Research Unit of Autoimmune Diseases, Sheba Medical Center, Tel Hashomer. and Sackler Faculty of Medicine, Tel Aviv University

Immune and inflammatory mechanisms have been consistently implicated in the pathogenesis of neurodegenerative diseases. Conversely, many immune mediated diseases of the central nervous system lead eventually to neurodegeneration Clinically, genes influencing neurodegenerative diseases, such as APOE, also influence the course of neuroimmunological diseases such as multiple sclerosis We have investigated whether immune challenges can modify the course of animal models of neurodegenerative diseases. The animal model chosen is a mouse strain carrying a pathogenic mutation in the $\beta A P P$ gene. The immune challenge used was induction of antiphospholipid antibodies by immunization with $\beta 2$-glycoprotein $I$. This immunization has previously been found to induce behavioral and cognitive dysfunction in a number of normal mouse strains, but not in C57BL, the background for the transgenic mice used in the present study. The mice were immunized at the age of 4-5 months and 4.5 months later were tested for hyperactivity and anxiety on a staircase apparatus and for cognitive function in a swim T-maze. There were significant differences in behavior between the APP transgenic mice compared to the controls in both tests. Induction of antiphospholipid antibodies impaired performance in the cognitive test only in the APP transgenic mice and had no significant effect in the wild type controls The immunization had no significant effect in the behavioral assay. These results indicate the potential role of immune mediated mechanisms in the pathogenesis of neurodegenerative processes and point to the potential use of immunomodulatory therapies in such diseases.

Keywords: transgenic mice; Alzheimer's disease; amyloid

precursor protein; $\beta 2$-glycoprotein-I; antiphospholipid antibodies

Molecular and pharmacological alterations of the serotonin receptor $\mathbf{5 H T}_{2 \mathrm{C}}$ in depression

Kinor N. ${ }^{1}$, Blatman G. ${ }^{1}$, Dremencov E. ${ }^{1}$, Overstreet D.H. ${ }^{2}$

Michaeli S. and Yadid G.

${ }^{I}$ Faculty of Life Sciences, Bar Ilan University; ${ }^{2}$ Dept. of Psychiatry, University of North Carolina, Chapel Hill

Serotonin ${ }_{2 \mathrm{C}}$ receptors $\left(5 \mathrm{HT}_{2 \mathrm{C}} \mathrm{R}\right)$ have been implicated in some psychiatric disorders, including depression. $5 \mathrm{HT}_{2 \mathrm{C}} \mathrm{R}$ undergoes editing whereby one to four specific adenosines are converted to inosines. Editing modulates receptor-G protein interactions thereby altering receptor-mediated signaling. We tested $5 \mathrm{HT}_{2} \mathrm{R}$ editing in the nucleus accumbens $(\mathrm{N}$. Acc) of Flinder sensitive line (FSL, an animal model of depression) and control rats using the sequencing method. FSL rats exhibit 50\% higher levels of the fully edited isoform (VSV) of $5 \mathrm{HT}_{2 \mathrm{C}}$ than control rats. Only control rats exhibited the non-edited isoform (INI) Treatment with desipramine had no effect on RNA editing although it improved swim behavior in FSL rats. Western blot analysis detected a decrease in protein levels of $5 \mathrm{HT}_{2 \mathrm{C}} \mathrm{R}$ in FSL rats after despiramine treatment although no significant changes were detected in mRNA and pre-mRNA levels of $5 \mathrm{HT}_{2 \mathrm{C}} \mathrm{R}$ in FSL rats or desipramine treated rats compared to controls. Acute exposure of the accumbal $5 \mathrm{HT}_{2 \mathrm{C}} \mathrm{R}$ to its antagonist, RS102221, resulted in dopamine release and this was doubled in FSL as compared to control rats. Therefore, we suggest that the $5 \mathrm{HT}_{2 \mathrm{C}} \mathrm{R}$ in the N.Acc has an inhibitory-like role in tonic DA release. Furthermore, $5 \mathrm{HT}_{2 \mathrm{C}}$ receptor RNA editing and pharmacology are altered in association with depressive behavior. These data indicate that $5 \mathrm{HT}_{2} \mathrm{C}$ receptor could be a potential target for future treatment of depressive behavior.

Keywords: RNA editing, serotonin- $2 \mathrm{C}$ receptor, microdialysis

Autoimmune neuroprotection: the evolutionary compromise needed to maintain autoimmunity on alert without the risk of autoimmune disease

Kipnis J., Avidan H., Mizrahi T., Lewitus G.M. and Schwartz M Weizmann Institute of Science, Rehovot 76100

Autoimmunity is the body's own mechanism against self-destructive compounds emerging as a consequence of CNS insult. In this work we show that induction of tolerance to self myelin-specific antigens, what was believed for years to be a preferential state of autoreactive cells, is against 'individual's fitness' in coping with stressful conditions after CNS injury. Neonatal immunization of rats with whole spinal cord homogenate, diminished the ability of adult rats to respond to myelin immunization and those animals show worse neuronal survival after optic nerve crush injury or spinal cord injury. As a corollary, immunization of adult animals with myelin-derived self-antigen or depletion of endogenous suppressor T cells (e.g. $\mathrm{CD} 4^{+} \mathrm{CD} 25^{+}$regulatory $\mathrm{T}$ cells) increased the animals' ability to resist injurious conditions. These findings call for the redefinition of tolerance to self-antigens, and for the role of CNS self-proteins as safeguards against CNS neurodegenerative disorders

Keywords: autoimmune neuroprotection, regulatory

$\mathrm{CD} 4^{+} \mathrm{CD} 25^{+} \mathrm{T}$ cells, CNS trauma, autoimmune diseases 
Perinatal dehydration correlates with adolescent salt preference

Kochli A. ${ }^{1}$, Rakover $\mathrm{Y}^{2}{ }^{2}$ and Leshem M. ${ }^{1}$

'Dept. of Psychology, Haifa University.; ' 2 Pediatric Endocrine Unit, Ha'Emek Medical Center, Afula

In our study of adolescents with congenital adrenal hyperplasia $(\mathrm{CAH})$ we find correlations between possible dehydrational events pre- and post-natal and the liking for salt taste expressed by adolescents. These events include scores of maternal vomiting and nausea when pregnant with the adolescent, and scores of the adolescent's levels of vomiting, diarrhea and infusions during infancy. We find positive and significant relationships between these events and how much the adolescent likes salt, years later. Since these data are based on recall by the mother of events occurring years previously, we attempted to control for the unreliability of the recall data by making comparisons within the family, i.e. comparing the recall data for siblings and its relationship to adolescent salt-liking. The relationship between perinatal dehydrational events and adolescent Finally, we find a strong relationship between maternal nausea and vomiting and the severest from of CAH. However, here the causal relationship seems to be reversed - apparently carrying a fetus with $\mathrm{CAH}$ induces higher levels of nausea and vomiting in the mother.

Keywords: human, salt, taste

\section{Licking of salt by adolescents with congenital adrenal} hyperplasia

Kochli A. ${ }^{1}$, Rakover $\mathrm{Y}^{2}$. and Leshem $\mathrm{M}^{1}$

'Dept. of Psychology, Haifa University; ${ }^{2}$ Pediatric Endocrine Unit, Ha'Emek Medical Center, Afula

The liking for salt in congenital adrenal hyperplasia (CAH) patients has been known in anecdotal form in the medical profession. However, there are no systematic reports of the phenomenon. In our study of adolescents with $\mathrm{CAH}$ we have reported that adolescents with the severest form of the disease, 'salt wasting', show a preference for salt as measured by questionnaires and psychophysical tests (Kochli, A. Rakover, $Y$. and Leshem, M. Neural Plasticity 8(3): 182; 2001). Here we present a semi-quantitative descriptive report of how these adolescents seek out pure salt, and discuss the issue of whether this behavior is an innate or acquired response to salt wasting. Keywords: human, salt, taste

\section{Activity-dependent translocation of the G-protein (DGq) in} Drosophila photoreceptors

KosToff M. Elia N., Timberg R. and Selinger Z

Dept. of Biological Chemistry, The Institute of Life Sciences

The Hebrew University, Jerusalem, 91904

The covalent lipid modification of proteins plays a major role in targeting heterotrimeric $(\alpha \beta \gamma) \mathrm{G}$ proteins to cellular membranes. In the case of the Drosophila visual system, palmitoylation of the cysteine residues at position 3 and 4 of an eye specific DGq-alpha is the sole lipid modification of the alpha subunit. Little is known, however, about the control of $\mathrm{G}_{\alpha}$ subunit localization within the natural endogenous environment of a specialized signaling cell. Here we show using live Drosophila flies, that light causes massive and reversible translocation of the visual $\mathrm{Gq}_{\alpha}$ to the cytosol, associated with marked architectural changes in the signaling compartment. Molecular genetic dissection together with detailed kinetic analysis enabled us to characterize the translocation cycle and to unravel how signaling molecules that interact with $\mathrm{Gq}_{\alpha}$ affect this process. Using specific visual mutants our results indicate that the translocation is not influenced by phototransduction steps at the level of PLC or downstream of it and that $\mathrm{Gq}_{\beta}$ is essential for efficient targeting of $\mathrm{Gq}_{\alpha}$ to the membrane.

Together with analysis of a 3-dimensional model of $\mathrm{Gq}_{\alpha}$ our in vivo results are mechanistically consistent with the "two signal model' for membrane targeting. Immuno-electron microscopy revealed that both $\mathrm{Gq}_{\alpha}$ and the signaling compartment undergo dynamic and reversible light-dependent changes. These events give $\mathrm{Gq}_{\alpha}$ access to other cellular compartments and point to possible cross talk between sensory transduction and the cytoskeleton.

Keywords: G-protein, localization, activity-dependent
Low GSK-3 $\beta$ in schizophrenia - a genetic marker or a neurodevelopmental insult consequence

Kozlovsky N. ${ }^{1}$, Nadri $\mathrm{C}^{1}$, Lipska BK ${ }_{1}^{2}$, Weinberger $\mathrm{DR}^{2}$, Shannon-Weickert $C^{2}$, Kleinman JE. ${ }^{1}$ and Agam G. ${ }^{1}$ ${ }^{I}$ Stanley Research Center, Ben-Gurion University of the Negev, Beer Sheva; ${ }^{2}$ Clinical Brain Disorders Branch, NIMH.

Bethesda MD, USA

Glycogen synthase kinase-3 (GSK-3) is a protein kinase highly abundant in brain and involved in signal transduction cascades, particularly neurodevelopment. Its activity and protein levels have recently been found over $40 \%$ lower in postmortem frontal cortex of schizophrenic patients. To elucidate whether the low levels are a genetic marker or a consequence of a perinatal neurodevelopmental insult the following experiments were carried out: GSK-3 $\beta$ protein levels in the frontal cortex of rats treated with neuroleptics or exposed to cold restraint stress were assayed. In the schizophrenia-related neonatal ventral hippocampal lesion rat model we measured GSK-3 $\beta$ protein levels and activity in the frontal cortex. To confirm our origina finding in another brain area we studied mRNA levels of GSK-3 $\beta$ in postmortem dorsolateral prefrontal cortex (DLPFC) from schizophrenic patients. Chronic treatments of rats with neuroleptics or exposure to cold restraint stress did not alter GSK-3 $\beta$ protein levels, supporting the concept that low GSK-3 $\beta$ in schizophrenia is not secondary to drug treatment or stress. However, GSK-3 $\beta$ protein levels in lesioned rats were significantly lower than in sham rats, favoring the perinatal insult possibility. GSK-3 $\beta$ mRNA levels were $36 \%$ lower in postmortem DLPFC of schizophrenic patients, supporting our previous findings. An additional intriguing recent preliminary finding is that GSK- $3 \beta$ protein levels in CSF samples from schizophrenic patients were found $28 \%$ lower than in control subjects. Further studies will be aimed at determining whether nonspecific neonatal damage or only specific factors cause low GSK-3 as a late effect

Keywords: schizophrenia, GSK-3 $\beta$, neurodevelopment

The cellular mechanisms underlying learning and stress Kogan I. and Richter-Levin G.

Dept. of Psychology, University of Haifa

Stress has multiple modes of influence on learning and memory, improving or impairing it under different conditions. The hippocampus is held accountable for explicit memory. The CA1 area of the hippocampus is reported to be involved in spatial memory. The amygdala is critically involved in mediating stress-related effects on behavior.

Memory storage has a late phase that requires protein synthesis. The activation of the MAPK (ERK) cascade is related to the establishment of the late, protein-synthesis dependent phase of memory formation. Thus, the activation of ERK and of its down-stream substrates may serve as a biochemical indicator for the activation of long-term memory processes.

Most learning protocols involve also a component of stress. In the present study we attempt to separate between learning and stress induced mechanisms by examining the activation of members of MAPK cascade (ERK1/2, CREB and Elk-1) in the hippocampus and amygdala. Three groups of rats were tested: 1. Learning - subjected to a spatial learning protocol. 2 Stressed - subjected to the water maze as the Learning group, but without an escape platform. 3. Naïve. Ten minutes after the last training session, tissue was collected for analysis of ERK, CREB and Elk-1 activation.

Only in the Learning group an activation of ERK was found in the CA1 area of the hippocampus but not in the amygdala Other molecules are still under the examination in both brain areas. The findings are expected to contribute to the dissociation between learning- and stress-induced mechanisms of signal transduction

Supported by The Israel Science Foundation - The Charles H.

Revson Foundation (no.582/00-1 to G.R-L.)

Keywords: memory, stress, protein synthesis

Molecular analysis of a novel neurodevelopmental rat model applying gene expression macroarray technology Koronyo-Hamaoui M. ${ }^{1,2}$, Gak E. ${ }^{1}$, Zuckerman L. ${ }^{3}$, Guetta E. ${ }^{1}$ Barkai G. ${ }^{1,2}$, Goldman B. ${ }^{1,2}$, Weizman A., ${ }^{2,4}$ and Weiner I ${ }^{3}$

${ }^{1}$ Danek Gertner Institute of Human Genetics, Sheba Medical

Center, Tel Hashomer: 'Sackler Faculty of Medicine. Tel Aviv

University, Tel Aviv, Israel. ${ }^{3}$ Psychobiology Unit, Dept. of

Psychology, Tel Aviv University, Tel Aviv: ${ }^{4}$ Felsenstein

Medical Research Center, Beilinson Campus. Petah Tikva

We have recently found that maternal immune activation during pregnancy by means of systemic administration of the synthetic cytokine releaser poly I:C in rats, led in the offspring to neurochemical and histological brain aberrations, as well as 
long term behavioral deficits (Zuckerman \& Weiner, submitted). Moreover, these deficits were not present before puberty but emerged at adulthood, implicating abnormal brain maturation processes. The present study used this model for the analysis of multiple gene expression profiles in two brain regions, the striatum and hippocampus, from the offspring of poly I:C treated and control dams. To this end, we employed Clontech cDNA macroarray technology containing 1200 rat genes relevant to neuroresearch and housekeeping genes. RNA preparations were pooled from four matched brain sections obtained from adult female offspring. RNA hybridization procedure was repeated in two independent trials.

Our data indicated that genes associated with synaptic trafficking and neuronal growth, synapsins (1A, B, 2A), syntaxin (B) and myelin basic proteins, were up-regulated in both striatum and hippocampus of the poly I:C offspring. In addition, several genes of the signal transduction pathway, G-coupled proteins and kinases, were elevated. Few other genes of the excitatory glutamate-NMDA pathway were up-regulated specifically in the poly I:C offspring striatum, while the poly I:C offspring hippocampus was distinct by elevated neuro-endocrine proteins secretogranin and somatostatin. Although these findings need further validation by other molecular methodologies, they may suggest that prenatal administration of poly $\mathrm{I}: \mathrm{C}$ interferes with neurodevelopmental processes involving neuronal remodeling, activity and proliferation, that might be associated with brain disorders such as schizophrenia.

Keywords: neurodevelopment, rat animal model, cDNA

expression arrays, poly I:C

Constitutive proteolytic activity is required for short-term plasticity of cultured Aplysia sensorimotor synapses Khoutorsky A. and Spira M.E.

Dept. of Neurobiology, Life Sciences Institute; The Hebrew University of Jerusalem, Jerusalem

The mechanisms underlying short term facilitation in Aplysia sensory-motor synapse can be subdivided into two processes: (a) a spike duration dependent process (sensitization) that results from 5HT induced PKA activation, and (b) a spike duration independent process (dishabituation) that results from PKC activation. As a result of PKA activation the potassium conductance is reduced, leading to spike broadening and enhanced calcium influx. The cellular mechanisms underlying PKC dependent synaptic dishabituation are not entirely understood. It was suggested that vesicles mobilization, alterations in the release mechanism or local activation of specialized calcium channels might be involved.

In contrast to earlier reports, we found that calpains (calcium activated cystein neutral proteinases), are involved in the cascade of events leading to synaptic habituation and dishabituation.

Application of the membrane permeable calpain inhibitors calpeptin or the nonspecific proteasome inhibitor MG132 increases the rate of synaptic habituation and inhibits $5 \mathrm{HT}$-induced synaptic dishabituation. On the other hand, sensitization is not affected by the inhibitors.

The results are consistent with the hypothesis that inhibition of constitutive proteolytic activity slow down or prevent the translocation of synaptic vesicles to the release sites. As a consequence the rate of synaptic habituation is accelerated in the presence of the inhibitors, and after massive habituation, $5 \mathrm{HT}$ application does not lead to dishabituation. These results demonstrate that constitutive proteolytic activity is necessary for the induction of short-term neuronal plasticity.

Keywords: synaptic habituation, dishabituation, proteolysis, Aplysia

\section{Hypothalamic plasticity: identification of genes involved in} thermal adaptation

Labunskav G., Shruster A., Yahav S. and Meiri N.

Institute of Animal Science, Agriculture Research

Organization, The Volcani Center, Bet Dagan 50250

The preoptic anterior hypothalamus (PO/AH) is responsible for body temperature control. This nucleus plays probably dual function both monitoring local temperature changes and integrating temperature information from the periphery. Thermoregulatory responses are controlled by thermo-sensitive neurons that change their firing rate in correlation with hypothalamic temperature. Thermal-conditioning in young animals cause a plastic change in the ratio of the aforementioned thermo sensitive neurons. Whereas in normal unconditioned $\mathrm{PO} / \mathrm{AH} 40 \%$ of the cells are temperature sensitive, after heat conditioning only $29 \%$ of the neurons are thermo sensitive. In this project we are identifying the gene products that are altered during thermal conditioning by using molecular techniques.

After thermal conditioning the AH/PO of young chicks was dissected in a time course ranging from minutes to 24 hours, and mRNA changes were monitored. We applied two strategies: 1. General mRNA amplification changes screening using differential display technique 2 . Since conditioning cause change in hypothalamic plasticity, we checked for changes in genes that are expected to be involved in growth and development concentrating on neurotrophic factors.

We identified 15 genes that are altered in the hypothalamus during heat conditioning. Among the induced genes we decided to further study the induction of M-RAS, 14-3-3 $\mathrm{N}-\mathrm{CAM}$ and BDNF, which are all related to growth and cellular interaction. The mRNA of M-RAS is maximally induced after 12 hours, and that of 14-3-3, N-CAM and BDNF after 6 hours. We are further analyzing the amount and exact location of the induction of these mRNAs.

Keywords: thermotolerance, chicks, differential display

Norepinephrine regulated genes involved in plasticity, CAM-L1 and CREB, are altered in post mortem brains of depressed patients

Laifenfeld D., Klein E., Karry R. and Ben-Shachar D. Dept. of Psychiatry, lab of Psychobiology, Rambam Medical Center and B.Rappaport Faculty of Medicine, Technion

Major depression has been shown to involve alterations in noradrenergic transmission. Recent research focuses on intracellular processes leading to synaptic plasticity as involved in the pathophysiology and treatment of depression. We have suggested a role for NE as a possible mediator of these processes. Previously, we reported NE-induced differentiation in SH-SY5Y cells accompanied by an increase in the cell adhesion molecule CAM-L1, in laminin and in CREB, all involved in processes of synaptic plasticity and neurite outgrowth. These genes were also increased in hippocampus and frontal cortices of rats treated with antidepressants, while decreased in a rat model of depression. In the present study we compared the expression of CAM-L1, laminin, CREB and p-CREB in post mortem brains of depressed patients $(n=15)$ bipolar patients $(n=15)$, schizophrenic patients $(n=14)$ and normal controls $(n=15)$ obtained from the Stanley Foundation Brain Bank. We found a two-fold increase in mRNA and protein levels of CAM-L1 in the frontal cortex of the depressed group compared to controls, which was not observed in either bipolar patients or schizophrenic patients. Likewise, CREB expression was increased 2.5 fold in depressed and 1.5 fold in bipolar patients, while pCREB was increased only in depressed patients, by 4 folds. No alterations were observed in laminin. No effect was found for post-mortem interval, age, age of onset, gender or antipsychotic medication. The present results support the hypothesis that noradrenergic dysregulation in depression leads to abnormal expression of genes involved in synaptic plasticity.

Keywords: depression, CAM-L1, CREB, post-mortem

Direction-specific effects of adaptation in motion-selective

neurons of cat primary visual cortex

${ }^{T}$ Dept. of Neurobiology, Weizmann Institute of Science, Rehovot 76100; ${ }^{2}$ Dept. of Neurobiology and Physiology, Northwestern University, Evanston, IL, USA

Adaptation, cause by prolonged visual stimulation, reduces the subsequent visual responses of neurons in primary visual cortex, as measured in both spiking and mean membrane potential. In previous works it was shown that adaptation induced membrane potential hyperpolarization of Vl neurons (Carandini et al. 1997). To distinguish between intrinsic mechanisms to network mechanism of adaptation we have studied the specificity of adaptation in visual responses of the neurons. If the mechanisms underlying adaptation are intrinsic to the recorded cell, adaptation should not change the selectivity of the neurons, but scale or reduce its response equally to all stimuli. We examine this hypothesis on direction selectivity in simple and complex cells. In simple cells we have found that adaptation, induced by drifting gratings, in either the preferred or the non-preferred direction cause a tonic hyperpolarization similar to what was previously described We did not measure, however, significant effect on the modulation of the response. In complex cells, on the other hand, we found a direction-specific effect of adaptation: Adaptation in the preferred direction caused a pronounced reduction in the direction selectivity assayed from the mean potential of the response. In contrast, adaptation in the non-preferred direction led to an increase in the direction selectivity of the neuron. The selectivity index of the 
non-adapted inputs, calculated from the responses to either direction after adaptation with the same direction of motion was no different than the control index, suggests that cortical adapting inputs do not change the selectivity of the cells. Furthermore, unless a significant fraction of the cortical inputs to direction selective cells are not affected by adaptation, intracortical activity does not make a significant contribution to direction selectivity

Supported by Grant R01 EY04726 from National Eye Institute

Keywords: intracellular recordings, in-vivo, direction

selectivity, cortical circuitry

Mismatch negativity (MMN) and "F (fusion) -complex" tap different aspects of deviance

Laufer I. and Pratt $\mathrm{H}$.

Evoked Potentials Laboratory, Technion-Israel Institute of

Technology, Haifa

The Mismatch negativity (MMN) component and the "F-(fusion) complex", the response to net-fusion, are brain potentials evoked by a deviant event. In this study we attempted to find out the extent to which these responses reflect the same process, albeit in different contexts.

The stimuli were base (presented to the front), that fused with the formant transitions presented to the front, left or right of the subject (the latter two producing an echo-sensation), resulting in a standard and deviant $\mathrm{V}-\mathrm{C}-\mathrm{V}$ sequences /aga/ and /ada/, respectively. Brain potentials were recorded from 10 normal hearing, right handed native Hebrew speakers, whose ages ranged between 19-30 years, that discriminated these V-C-Vs. Low resolution electromagnetic tomography (LORETA) t-test comparison images, that were run on the responses to deviants, showed that in case of front-fusion (no echo-sensation) activity contributing to the MMN was larger between 191-218 msec and was localized to the temporal cortices (BA 21,22). In this case, the phonetic identity of the auditory object affected the MMN. In contrast, an enhanced "F-complex" was associated with the lateralized fusion conditions that sounded different in spatial attributes (echo). This activity was localized to parieto-temporal (BA 19,39), occipital (BA 31) and frontal (BA 9, 10) regions in the latency range of 226-296 msec.

These results indicate that the MMN and "F-complex" tap different aspects of deviance: the "F-complex" is affected by properties of the auditory object itself, whereas the MMN is affected by its novelty in relation to other stimuli.

Keywords: speech, auditory evoked potentials (AEPs), fusion MMN, "F (fusion)-complex"

\section{Cannabinoid CB2 selective PRS-211,096 reduces} neurological deficit in experimental autoimmune encephalomyelitis (EAE)

Lavie V., Bar-Joseph A., Dar D.E., Garzon A., Menashe N., Margalit R. and Fink $G$.

Pharmos Ltd, Kiryat Weizmann, Rehovot 76326

Dexanabinol (HU-211) a nonpsychotropic cannabinoid has been shown to reduce the clinical score in rats with EAE. PRS-211,096, a novel bicyclic cannabinoid, binds with high affinity to the peripheral cannabinoid $-2(\mathrm{CB} 2)$ receptor $\left(\mathrm{IC}_{50}=\right.$ $0.42 \mathrm{nM})$ and with lower affinity $\left(\mathrm{IC}_{50}=22 \mathrm{nM}\right)$ to the central CB1 receptor. $C B 2$ receptors are expressed mainly by $B$ and $\mathrm{CD}_{4}{ }^{+}$and $\mathrm{CD}_{8}{ }^{+} \mathrm{T}$ cells as well as monocytes, and are known to be involved in immunomodulation. The aim of the present study was to determine whether PRS-211,096 could reduce the severity of clinical signs in EAE rats. EAE was induced in Lewis female rats by injecting (s.c) myelin basic protein (MBP) in complete Freund's adjuvant. After the first appearance of clinical signs, PRS-211,096 was administered on three consecutive days at different doses. The severity of the illness was determined by the mean maximal increment in the severity of the clinical score. The significance of differences between groups was determined by ANOVA followed by Fisher's LSD The results showed that PRS-211,096 significantly reduced clinical score and alleviated illness severity in a dose-dependent manner (22-33\% compared with that in vehicle treated animals). Depending on dosage, the efficacy of PRS-211,096 was similar or greater than that of methyl prednisolone (20\% compared with vehicle). Interferon-beta (Betaseron), and IgG were ineffective. The efficacy of PRS-211,096 in reducing the neurological deficit in EAE seems likely to be due at least in part to its affinity for the CB2 receptor. Thus, PRS-211,096 provides the basis for developing novel drugs for the treatment of multiple sclerosis in the human.

Keywords: multiple sclerosis, EAE, neuroprotection
Neuroprotection by PRS-211,220: assessed functionally and morphologically in transient MCA occlusion rats Lavie V., Bar-Joseph A., Berckovitch Y., Weksler A., Azulay M., Grienstein Y., Garzon A., Amselem S. and Fink G. Pharmos Ltd, Kiryat Weizmann, Rehovot 76326

Dexanabinol (PRS-211,007) is a non-psychotropic cannabinoid, which acts as a noncompetitive NMDA receptor antagonist, and has anti-oxidant and anti-inflammatory activities. It was shown to be neuroprotective in brain ischemia and traumatic brain injury models. PRS-211,220 is a novel analog of Dexanabinol, with two times higher affinity for the NMDA receptor and 30 times better ability to inhibit cyclooxygenase-2 than Dexanabinol. The aim of the present study was to determine whether PRS-211,220 had long-term beneficial effects on functional outcome and on infarct volume following focal brain ischemia. The middle cerebral artery (MCA) of adult Sprague Dawley rats was occluded for 120 minutes by intraluminal suture under halothane anesthesia. PRS-21 1,220 $(0.1,0.25,0.5,1,2.5,5$ or $10 \mathrm{mg} / \mathrm{kg}$ IV $)$ and its vehicle were administered by the end of the ischemia. The neuroprotective efficacy of the compound was evaluated behaviorally by the "staircase test" and morphologically by measuring infarct volume. Rats were trained for 1 week prior to the insult, twice a day for 15 minutes. Thereafter, rats were tested for 2-3 weeks. By the end of the last test brains were removed, serially sectioned and stained with thionin. Infarct volumes were evaluated using a computerized image analyzer. PRS-211,220 induced a dose related improvement in staircase test performance on the contralateral side. (30-55\% compared with vehicle alone, $55 \%$ improvement at $0.5 \mathrm{mg} / \mathrm{kg} \mathrm{p}<0.05$,). Infarct volume was also reduced with PRS-211,220 (44\% at $0.5 \mathrm{mg} / \mathrm{kg}$ ). PRS-211,220 induces functional as well as morphological neuroprotection following transient MCAo in rats.

Keywords: neuroprotection, stroke, staircase test, dexanabinol

The temporal evolvement of local and global image representations in human high order object areas Lerner $Y^{1}$, Harel M. ${ }^{1}$, Hendler $T .{ }^{2}$ and Malach $R^{1}$

Weizmann Institute of Science, Rehovot 76100; ${ }^{2}$ Sourasky

Medical Center, Tel Aviv 64239

Recently we have found that human high-order object areas are driven partially by local object fragments, and partially by global completion effects. Here we studied to what extent the local and global effects might emerge at different rates. We studied the temporal dynamics of these effects using brief exposures through a backward masking paradigm. In the first experiment, line drawings of animal shapes were shown that were occluded by parallel, vertical bands ("grid"), or scrambled by randomizing the relative location of the object stripes ("scrambled"), each presented in 2 different exposure durations $(60 \mathrm{~ms}, 250 \mathrm{~ms})$. In the second experiment, the same objects were presented either without occlusions ("whole") or with the "grid" condition, again using 60 and $250 \mathrm{~ms}$ exposures. The results showed that for both exposure durations, in occipito-temporal (LO and pFs) cortex, the signal in the "grid" condition was higher than the "scrambled" in the first experiment, and the "whole" condition exceeded the "grid" in the second one. However, the ratio between the preferred and non-preferred conditions in each experiment was similar at 60 $\mathrm{ms}$ and $250 \mathrm{~ms}$. The activation pattern matched very well the recognition performance of the subjects. In conclusion, our results show that the emergence of global completion effects is as rapid as the emergence of local feature representation. Thus, as early as $60 \mathrm{~ms}$ - when the visual activation is still low, the relative contribution of completion effects is already similar to that found for longer exposure times.

Supported by Israel Academy 8009/00-1 and MP 6971 grants. Keywords: fMRI, visual system, object recognition, completion

Resistance of bax-deficient mice to MOG-induced experimental autoimmune encephalomyelitis (EAE) Lev N Barhum Y, Melamed E and Offen D.

Felsenstein Medical Research Center, Dept. of Neurology, Rabin Medical Center. Tel Aviv University, Sackler School of Medicine, Petah-Tikva 49100, doffen a.post.tau.ac.il

Multiple sclerosis (MS) is an inflammatory disease of the central nervous system (CNS) characterized by destruction of myelin. Recent studies indicate that accumulating axonal damage is involved in the pathogenesis of progressive disability and frequently occurs in this disease. To study the role of axonal damage in the pathogenesis of MS-like disease induced by myelin oligodendrocyte glycoprotein (MOG), we compared experimental autoimmune encephalomyelitis (EAE) in wild type (WT) and transgenic mice deficient in bax, a 
pro-apoptotic gene. Our study shows that following EAE induction with pMOG 35-55, the WT mice developed significant clinical manifestations with complete hind limb paralysis (average score $2.3 \pm 0.5$ ). In contrast, most of bax-deficient mice were resistant and did not develop the disease, while others showed only mild clinical signs (average score $1.02 \pm 0.32, \mathrm{p}<0.05)$. No difference could be detected in the immune potency as indicated by $T$ cell proliferative responses of WT and bax-deficient mice to MOG and to Con A. In conclusion, knock-out of the pro-apoptotic gene bax, attenuates the severity of MOG-induced EAE. Our results emphasize the importance of apoptosis in the development of EAE and most probably also in MS

Keywords: multiple sclerosis, Bax, transgenic mice, axonal damage

Effect of systemic inflammation during pregnancy on maternal and offspring behavior

${ }_{\mathrm{Lev}^{1,4}}{ }^{1,4}$, Huleihel M. ${ }^{2}$, Hallak M. ${ }^{3}$, Sorokin $\mathrm{Y}^{5}$ and Golan Depts. of Developmental and Molecular Genetics ${ }^{l}$, Dept. of Immunology and Dept. of Obstetrics and Gynecology, Faculty of Health Sciences and Zlotowski Center for Neuroscience, Ben-Gurion University, Beer-Sheva; ${ }^{4}$ Dept. of Obstetrics and Gynecology, Hutzel Hospital, Detroit,

Michigan, USA

Intrauterine inflammation is associated with high levels of pro-inflammatory cytokines in maternal blood. To examine the damage of high levels of inflammatory cytokines on brain development, systemic maternal inflammation was induced by intraperitoneal (i.p) injection of lipopolysaccharide (LPS, $0.12 \mathrm{ug} / \mathrm{gr}$ ) to 17 gestation day mice. As we previously reported, $3 \mathrm{~h}$ following LPS injection, IL-1 $\beta$ levels were increased by three folds in embryo brains, and remained high for 6-12 hours.

Under these conditions no difference in the delivery day was observed between LPS and control groups (vehicle). Among the LPS- injected mothers (LPS-M, $n=27$ ), 44\% did not exhibit maternal behavior lead to newborn death and $18 \%$ exhibit canibalism as compared to $10.5 \%$ and $10.5 \%$ respectively, in the control group $(n=19)$. We have previously reported that LPS-M did not affect newborns weight, time of teeth eruption and hair growth. However, in contrast to our previous observation, detailed examination reveal accelerated eyelid opening in the LPS-M group as compared to control; at postnatal day 14 (P14) only $33 \%$ and $81 \%$ (P15) of the control mice opened their eyelids, while at the LPS-M group 55\% (P14) and $100 \%$ (P15) already demonstrate open eyelid $(n=16-30, p=0.0001)$. The righting reflex developed earlier in the offspring of LPS-M than in the controls ( $n=7-32$, $\mathrm{p}<0.0001$ ). However, no difference related to muscle strength and coordination development. At P70 the performance of LPS-M group in the open-field was similar to control group as measured by margin/center ratio and number of moves. Disturbance in motor skills of newborns of LPS-M was detected by a low variance of step size examined in the hinpaw footprint $(p<0.027)$. Based on these observations, we suggest that systemic maternal inflammatory response induce neurodevelopmental alterations, which may influence some aspects of the animal performance.

Supported by BSF grant contract No. 200172

Keywords: IL-1b, cytokines, LPS, development

ApoE4 impairs hippocampal plasticity isoform-specifically and blocks environmental stimulation of synaptogenesis and memory

Levi $O$ and Michaelson DM.

Faculty of Life Sciences, Tel Aviv University, Tel Aviv

Alzheimer's disease (AD) is associated with genetic risk factors, of which the allele E4 of apolipoprotein $E$ (apoE4) is the most prevalent, and with environmental factors that include early life education and socio-economic background. The extent to which environmental factors affect the phenotypic expression of the $\mathrm{AD}$ genetic risk factors is not known. We have recently shown that exposure of apoE transgenic mice to enriched environment at a young age, elicits apoE genotype specific cognitive effects. Accordingly, exposure to an enriched environment of mice transgenic for human apoE3, which is the benign $A D$ apoE allele, results in improved learning and memory, whereas mice transgenic for human apoE4 are not so affected by the enriched environment. In the present study we examined the effects of the enriched environment on brain neuronal parameters in the apoE transgenic mice. Measurements of the presynaptic protein synaptophysin and of NGF in the hippocampus of the transgenic mice revealed that environmental stimulation induced marked elevation in the hippocampal synaptophysin and NGF levels of the apoE3 transgenic mice whereas those of the apoE4 transgenic mice were not affected by this treatment. In contrast, the cortical synaptophysin and NGF levels of the apoE3 and apoE4 transgenic mice were similarly elevated by environmental stimulation. These findings show that apoE4 impairs hippocampal plasticity isoform-specifically and blocks the environmental stimulation of synaptogenesis and memory This provides a novel mechanism by which environmental factors can modulate the function and phenotypic expression of the apoE genotype.

Keywords: ApoE, plasticity, enriched environment,

Alzheimer's disease, working memory, synaptogenesis

Retinotopic organization of human visual areas following a bilateral retinal scotoma

Levin N. ${ }^{1}$ and Zohary E. ${ }^{2}$

${ }^{7}$ Dept. of Neurology, Hadassah - Hebrew University Hospital, Jerusalem; ${ }^{2}$ Neurobiology Dept., Hebrew University, Jerusalem In contrary to long held beliefs, sensory and motor maps are not immutable in adult cerebral cortex. In the monkey visual system, cortical reorganization has been demonstrated following focal retinal lesions. Thus, neurons that have receptive fields within the lesioned visual field (i.e. scotoma) undergo a functional change so that their receptive field shifts from representing the lesioned part of the retina to visual areas surrounding the lesion. To what extent does such neuronal plasticity exist in humans? We utilize the fact that fine retinotopic mapping of the visual field has been convincingly demonstrated using fMRI. Thus, it is feasible to detect cortical remapping of the visual field following topographically restricted damage to the retina. Functional MRI signals were obtained in a subject (DT, age 42 years) suffering from bilateral lower altitudinal scotoma. A contrast-reversing wedge rotating around the fixation point was used to measure the retinotopic organization with respect to polar angle Surprisingly, the upper calcarine sulcus that normally represents the lower visual field (that the subject cannot see) was activated by the visual stimulus.

Thus, the pattern of activation reported here does not conform to the classical pattern of neuronal plasticity found in monkeys. The activation in the cortical regions representing the blind field may be interpreted as (1) expectation of the stimulus in the blind field due to its cyclic appearance (2) existing islands of activity in the retinal blind area, which are not detected by classical perimetry methods.

Keywords: fMRI, vision, mapping, scotoma

\section{A dominant negative inhibitor of the Egr family of transcription regulatory factors suppresses cerebellar granule cell apoptosis by blocking c-Jun activation Levkovitz Y. and Baraban JM \\ ${ }^{7}$ Shalvata Mental Health Center, School of Medicine, Tel-Aviv University; ${ }^{2}$ Depts. of Neuroscience, Psychiatry and Behavioral Sciences, Johns Hopkins University School of Medicine, Baltimore, USA}

To investigate the role of the Egr family of transcription regulatory factors in neuronal apoptosis, we examined the effect of a dominant negative Egr inhibitor construct in a well characterized in vitro paradigm, cerebellar granule cell death induced by withdrawal of depolarizing concentrations of extracellular potassium. We found that this apoptotic stimulus increases the activity of a reporter gene driven by the Egr response element and that a dominant negative inhibitor of Egr-mediated transcription blocks granule cell apoptosis. In contrast, apoptosis of immature granule cells induced by cytosine arabinoside is not inhibited by the Egr inhibitor construct. Because activation of c-Jun is an essential step in granule cell death induced by potassium deprivation, but not cytosine arabinoside, we asked whether the Egr inhibitor acts by influencing c-Jun activation or its ability to induce apoptosis. We found that the Egr inhibitor does not block the ability of a constitutively active c-Jun construct to induce apoptosis in these cells but does suppress activation of c-Jun-mediated transcription induced by lowering extracellular potassium concentration. Furthermore, the Egr inhibitor blocks the ability of MEKK1 [mitogen-activated protein kinase (MAPK) kinase kinase 1], an upstream kinase capable of stimulating the JNK (c-Jun N-terminal protein kinase)-c-Jun pathway, to induce apoptosis and activate c-Jun. Together, these studies indicate that the Egr family of transcription factors plays a critical role in neuronal apoptosis and identify c-Jun activation as an important downstream target of the Egr family in this process.

Keywords: apoptosis, transcription factors, Egr family, c-Jun 
Continuous representation of objects in the human posterior fusiform gyrus

Levy I. ${ }^{1,2}$, Witz S. ${ }^{3}$, Hasson $U_{.}^{2}$, Hendler T. ${ }^{4,5}$ and Malach $R^{2}$ ${ }^{7}$ Hebrew University of Jerusalem, Jerusalem 91904;

${ }^{2}$ Weizmann Institute of Science, Rehovot 76100; ${ }^{3}$ Beer Ya'acov Mental Health Center, Beer Ya'acov; ${ }^{4}$ Sourasky Medical

Center, Tel Aviv 64239; ${ }^{5}$ Tel Aviv University, Tel Aviv 69978

The human ventral occipito-temporal cortex exhibits complex activation patterns to different objects. Recent studies revealed some of the principles which may underlie these representations, e.g. resolution needs and expertise level. However, the representation of objects along these dimensions may be either modular (i.e. each stimulus type activates a distinct region) or continuous (i.e. activations to different stimulus types slide smoothly along a certain dimension). The two possibilities lead to different predictions: in the modular case different objects should either activate the same area or different areas, whereas in the continuous case activations to different objects will be partly overlapping and partly offset compared to each other. To test these predictions we conducted a functional MRI experiment, in which subjects (9) were presented with head images in front and back views. Images were presented in 9-sec single-category epochs, interleaved with 6-sec periods of blank screen. We obtained activations of highly overlapping yet slightly offset regions in the fusiform gyrus. Crucially, the direction of this displacement was consistent across subjects and therefore cannot be attributed to random variability; In addition, the non-overlapping strips of activation were not the regions of highest activation to each category, excluding low-resolution, or partial volume effects as the cause of the overlap. We conclude that the activation pattern reflects a continuous topography, in which representations of objects "slide" smoothly along the fusiform gyrus.

Supported by ISF 8009

Keywords: visual cortex, fMRI, object recognition, topography

Adult human bone marrow-derived mesenchymal stem cells differentiate into neural cells

Levy Y.S. ${ }^{1}$, Bulvik S. ${ }^{2}$, Burshtein A. ${ }^{1}$, Barhum Y. ${ }^{1}$, Melamed

E. and Offen D.

${ }^{1}$ Felsenstein Medical Research Center, Dept. of Neurology,

Rabin Medical Center, Tel Aviv University, Sackler School of Medicine, Petah-Tikva: ${ }^{2}$ Laniado Medical Center. Natanya:

doffeniajpost.tau ac.il

Human mesenchymal stem cells present in adult marrow, are believed to be multipotent and can develop into bone, cartilage, fat, tendon and muscle. Here, we demonstrate that human bone marrow stromal cells (hBMSc) can be induced to differentiate into neural cells in vitro. Isolated hBMSc from human iliac crest incubated with retinoic acid, butylate hydroxyanisole and increased intracellular cyclic AMP, changed their phenotype to neural cells. The differentiated cells were positive for nestin, neurofilament-H (NF-H), neuron-specific enolase (NSE) proteins, neural nuclei (NeuN) as indicated by immunocytochemistry and Western blot assays. The mRNAs for NSE, retinoic acid receptor and neurite outgrowth promoting protein (NEGF2) were identified in differentiated and undifferentiated hBMSc using reverse transcriptase polymerase chain reaction. However, mRNAs for Musashi-1, necdin and NF-H were detected only in differentiated cells The mRNA expression of NEGF2 was increased during differentiation, as assessed by real-time PCR. In conclusion our method might offer a new and more accessible source of neural cells for transplantation to treat neurodegenerative diseases.

Keywords: human bone marrow stromal cells (hBMSc), nestin, Neurofilament-H (NF-H), Neuron-specific enolase (NSE), Neural nuclei (NeuN)

\section{Anticonvulsant action of bromide is associated with} enhanced synaptic inhibition

Libman L. , Astman N. ${ }^{2}$, Fleidervish I.A. ${ }^{1}$, Heinemann U. ${ }^{3}$ and Gutnick M.J. ${ }^{1}$

${ }^{I}$ Koret School of Veterinary Medicine, The Hebrew University of Jerusalem, Rehovot, ${ }^{2}$ Zlotowski Center for Neuroscience, Ben-Gurion University of the Negev, Beersheva \& ${ }^{3}$ Dept. of Physiology, Humboldt University, Berlin

Bromide salt $(\mathrm{Br})$ was introduced as a treatment for epilepsy in the 1850's, making it the first anticonvulsant drug (AD) Although $\mathrm{Br}$ has been almost entirely superceded by newer $\mathrm{ADs}$, it is still used on occasion in humans and more frequently in veterinary medicine. Yet, the mechanism underlying the anticonvulsant action of this halogen is not known. Because most ADs target either neuronal Na channels or GABAergic inhibition, we have investigated the effect of $\mathrm{Br}$ on these determinants of circuit excitability in in-vitro preparations of rodent neocortex. In organotypic cortical slice cultures, $\mathrm{Br}(20$ $\mathrm{mM}$ ) reversibly blocked the epileptogenic activity induced by removal of $\mathrm{Mg}$ from the bath solution. In whole cell patch-in-slice recordings from neocortical pyramidal neurons $\mathrm{Br}$ application did not have any apparent affect on $\mathrm{Na}$ channel availability. Thus, in current-clamp, it did not affect thresholds, amplitudes or maximum rates of rise of single action potentials, nor did it compromise neuronal capacity to fire at high frequencies. $\mathrm{Br}$ also did not cause significant changes in passive membrane parameters. By contrast, $\mathrm{Br}$ did have a profound effect on GABA-A receptor mediated inhibition. In voltage-clamp recordings, it prolonged by $>30 \%$ the decay time constants of mIPSCs, sIPSCs and stimulus-evoked IPSCs, resulting in a considerable increase in inhibitory charge transfer. The mechanism for this has yet to be determined; it is probably related to Br's greater permeability through the chloride channel. We conclude that like barbiturates and benzodiazepines, $\mathrm{Br}$ achieves its anti-convulsant effect by enhancing synaptic GABAergic inhibitory activity.

Keywords: anticonvulsant, epilepsy, $\mathrm{Na}$ current, neocortical neuron, IPSC

Low socio-economic status, a risk factor for ischemic stroke. A case-control study in Southern Israel Lieberman T. ${ }^{1}$, Herishanu Y.O. ${ }^{1}$ and Kordysh E ${ }^{2}$ ${ }^{T}$ Neurology Dept., Soroka University Medical Center Beer Sheva: ${ }^{2}$ Epidemiology Unit, Ben-Gurion University of the Negev, Beer-Sheva

Background: The risk factors for ischemic stroke (IS) are age, hypertension, ischemic heart disease, atrial fibrillation, diabetes, dyslipidemia, hypercoagulopathy, smoking, carotid artery stenosis, TlA etc. An additional risk factor with a variable impact is a low socio-economic status.

Aim: To study the role played by the socio-economic status in the etiology of IS in the heterogeneous population of the Negev (Southern Israel)

Methods: 102 acute ischemic stroke patients were compared to $102 \mathrm{sex}$, age and country of origin matched patients admitted to different surgical departments. All the patients were interviewed about medical history, habits, socio-economic status, education, past and present occupation, income, living conditions, property ownership, medical insurance, social support. STATA was used for statistical analysis. For univariate and multivariate analysis, $\mathrm{X} 2$, $\mathrm{t}$ test, univariate and multivariate logistic regression with Odd Ratio and $95 \% \mathrm{CI}$ calculation.

Results: A significant association of low socio-economic status and IS incidence was found. On univariate analysis a significant positive association was found with spouse low educational level $(\mathrm{p}=0.05$ ), spouse employment in blue-collar occupation $(p=0.047)$ and with low income $(p=0.0017)$. The IS patients were less property owners $(p=0.000)$ and lived in crowded conditions $(p=0.027)$, without additional medical insurance $(p=0.025)$ and without emotional support $(p=0.037)$ and without friends $(p=0.000)$. On multivariate analysis, systolic hypertension heart disease, diabetes, family history of hypertension and low income were found to be risk factors for IS.

Conclusion: Low socio-economic status is associated with an increased risk for IS. People with a low level of education and income is the target population for intervention on known medical risk factors to prevent IS

Keywords: ischemic stroke, socio-economic status, income, risk factors

\section{Assay of GTP hydrolysis by G-proteins}

Litvak Y., Kosloff $M$. and Selinger $Z$

Dept. Of Biological Chemistry, The Institute of Life Sciences, The Hebrew University of Jerusalem, Jerusalem, 91904

G-proteins are transducers of a wide range of cellular transactions, including transmembrane signaling, cell proliferation, intracellular transport and modulation of cytoskeletal organization. Despite their functional diversity, all members of the G-protein family share a common regulatory mechanism, the so-called regulatory GTPase cycle. The interaction of G-proteins with their downstream effectors is determined by the conformational state of the G-protein, which is influenced by the identity of the guanine-nucleotide in the binding site. When charged with GTP (guanosine triphosphate), the G-protein is in the 'on state', capable of acting on its downstream effectors. Hydrolysis of the bound GTP to GDP (GTPase), causes the G-protein to lose this ability, and 'turns the protein off'. This cycle constitutes the G-protein's biochemical on/off switch. The challenge in designing a GTPase assay is to enable us to determine the net 
rate of GTP hydrolysis. This is not a trivial undertaking because both the on and off reactions occur simultaneously and GTP hydrolysis is not necessarily the rate-limiting step. Here we present the development of a GTPase assay that overcomes these difficulties. It allows us to investigate the effect of different molecules and protein modifications on the rate of GTP hydrolysis by G-proteins.

Keywords: signal transduction, ras protein, one cycle GTPase assay

\section{Computation By Ensemble Synchronization In Recurrent} Networks With Synaptic Depression

Loebel A. and Tsodyks M.

Dept. of Neurobiology, Weizmann Institute of Science, Rehovot 76100

While computation by ensemble synchronization is considered to be a robust and efficient way for information processing in the cortex (C. Von der Malsburg and W. Schneider Biol. Cybern. 54: 29-40 [1986]; W. Singer Inter. Rev. Neuro. 37: 153-183 [1994]; J.J. Hopfield Nature 376: 33-36 [1995]; E. Vaadia et al. Nature 373: 515-518 [1995]), the neuronal mechanisms that might be used to achieve it are yet to be uncovered. Here we analyze a neural network model in which the computations are performed by near coincident firing of neurons in response to external inputs. This near coincident firing is enabled by activity dependent depression of inter-neuron connections. We analyze the network behavior by using a mean-field approximation, which allows predicting the network response to various inputs. We demonstrate that the network is very sensitive to temporal aspects of the inputs. In particular, periodically applied inputs of increasing frequency result in different response profiles. Moreover, applying combinations of different stimuli lead to a complex response, which cannot be easily predicted from responses to individual components. These results demonstrate that networks with synaptic depression can perform complex computations on time-dependent inputs utilizing the ability to generate temporally synchronous firing of single neurons.

Keywords: mean field, population spike (PS), recurrent network, synaptic depression

\section{Behavioral responses to pain of individuals with cognitive} disability

Lotan M. ${ }^{1}$, Pick C.G. ${ }^{1}$ and Defrin R. ${ }^{2}$

Dept. of Anatomy and ${ }^{2}$ Dept. of Physiotherapy, Sackler School of Medicine. Tel-Aviv University, 69978, Tel-Aviv.

Introduction: Individuals with cognitive impairment as well as the entire population are exposed to painful medical procedures and painful conditions. However, since many of them cannot communicate verbally with the surrounding, they are frequently undertreated for pain. Pain behavior scales that do not necessitate verbal reports have only recently been used for this population. Our aim was to study whether the behavioral responses to pain differ according to the level of cognitive impairment and whether these are in accordance with verbal reports of pain.

Methods: 108 cognitively impaired individuals (22 mild; 43 moderate; 23 severe and 24 profound) participated. They were videotaped before and during a flu vaccination. Two examiners, using two behavioral scales, analyzed pain behavior: NCCPC-R (general pain behaviors) and FACS (facial expression of pain). Subjects with mild and moderate cognitive impairment were also asked to rate their perceived pain on a VAS scale.

Results: Pain behavior, measured with NCCPC-R, was significantly increased in all groups during the injection $(p<0.001-0.01)$, while facial expression of pain (FACS) was only significantly increased in the mild and moderate retardation groups $(\mathrm{p}<0.01), 30 \%$ of individuals with severe to profound retardation responded with a "frees" during injection compared to only $4.5 \%$ of individuals with mild to moderate retardation. There was a good correlation $(r=0.76)$ between the VAS and the behavioral scores only in individuals with mild retardation. In addition, there was a good to high correlation between the two methods $(r=0.60-0.87)$ and a high correlation between the two examiners ( $r=0.91-0.97$ ).

Conclusion: Both NCCPC-R and FACS reliably detect changes in pain intensity in individuals with mild to moderate retardation but NCCPC- $R$ is more sensitive to these changes compared to FACS in sever to profound retardation. Both scales have high intra rater reliability. The response to acute pain differs according to the level of retardation.

Keywords: pain, mental retardation, pain behavior
The neurosteroid DHEA attenuates cocaine-seeking behavior in rats

Lotan S. ${ }^{1}$, Maayan R. ${ }^{1}$, Weizman A. ${ }^{1}$, Yadid G. ${ }^{2}$

${ }^{7}$ Felsenstein Medical Research Center, Beilinson Campus

Petah-Tikva, Sackler Faculty of Medicine, Tel-Aviv University; ${ }^{2}$ Faculty of Life Sciences, Bar-Ilan University

The term "neurosteroids" refers to steroids that can be synthesized de novo in the nervous system from sterol precursors. The group includes pregnenolone (PREG) dehydroepiandrosterone (DHEA), their sulfates (PREG-S and DHEA-S) and reduced metabolites such as tetrahydroprogesterone (allopregnanolone). In addition to the well known genomic effect of steroids via nuclear receptors, neuroactive steroids can also act as allosteric modulators of neurotransmitter receptors, such as $\mathrm{GABA}_{A}$, NMDA, and sigma receptors, and thus affect behavior. Indeed, clinical studies in humans have associated some of these hormones with a sensation of "well-being", but also with reward-related processes, mood and motivation. Neurosteroids thus may play a role in substance abuse. In the present study, we tested the hypothesis that the neurosteroid DHEA attenuates cocaine self-administration. Rats were pretreated with either DHEA (2 $\mathrm{mg} / \mathrm{kg} /$ day i.p.) or vehicle solution, and were then trained to self-administer cocaine $(1 \mathrm{mg} / \mathrm{kg} /$ infusion $)$ on a fixed-ratio schedule of reinforcement, while continuing hormone treatment. DHEA significantly reduced cocaine-seeking behavior. These results suggest that DHEA affects cocaine reward. We further analyzed brain DHEA levels in non-treated rats after exposure to cocaine. Our data show a 2-3 fold increase in DHEA levels in different mesolimbic brain areas of rats that maintain drug-seeking behavior compared to sham-operated controls.

This suggests that changes in brain concentrations of neurosteroids may play a role in the modulation of psychological states, including cocaine dependence.

Keywords: neurosteroids, DHEA, cocaine, self-administration

Neurosteroids modulating the antidepressive activity of

paroxetine ${ }^{1}$, Spivak B ${ }^{2}$, Ram E. ${ }^{1}$ and Weizman A. ${ }^{1}$

${ }^{T}$ Laboratory of Biological Psychiatry, Felsenstein Medical Research Center, Beilinson Campus; ${ }^{2}$ Research Unit Ness Ziona Mental Health Center, Ness Ziona

Although it is known that selective serotonin reuptake inhibitors (SSRIs), as other antidepressants, elevate mood only after 3-4 weeks of treatment the mechanism responsible for this delay is not understood. SSRIs have been demonstrated to alter the levels of neurosteroids such as allopregnanolone (THP) which possess anxiolytic and mood elevating properties We compared the effect of 9 and 21 days i.p. administration of paroxetine, a potent SSRI, on the synthesis of THP and its precursor, $5 \alpha$-dihydroprogesterone (DHP) in the mouse cortex hypothalamus and olfactory bulb. Cortex, olfactory bulb and hypothalamus synthesized levels of DHP were significantly raised after 9 days of paroxetine administration, whereas a significant rise in the THP synthesized level was observed only after 21 days of treatment. Peripheral synthesis of DHP measured by the level in serum, significantly increased after 9 days, but reverted to normal values after 21 days. No increase was detected in serum THP levels either after 9 or 21 days treatment. Differences in peripheral and brain synthesis indicates independence in brain synthesis. The data indicate that paroxetine administration differentially increases $\left[{ }^{3} \mathrm{H}\right] \mathrm{DHP}$ and THP content, depending on the duration of the treatment Our results suggest that brain THP may be involved in the antidepressive and anxiolytic activity of paroxetine.

Keywords: paroxetine, neurosteroids, allopregnanolone (THP)

Nicotine switches on "silent" synapses in the developing hippocampus

Maggi L. ${ }^{1}$, Le Magueresse C. ${ }^{1,2}$, Changeux J.P. ${ }^{2}$ and Cherubini

E

${ }^{1}$ Neuroscience Program, International School for Advanced Studies (SISSA), Via Beirut 2-4, 34014 Trieste, Italy. ${ }^{2}$ Unit. de Neurobiologie Moleculaire, Institut Pasteur, 25-28 Rue du Docteur Roux, 75724 Paris Cedex 15, France

Synapses are "silent" either because neurotransmitter is not released or because they are unable to detect the release of neurotransmitter, due to the lack of postsynaptic receptors. Recently we have shown that in the hippocampus of new-born rats, increased release of glutamate rather than insertion of new postsynaptic AMPA receptors account for switching on silent synapses and for LTP maintenance in both CA1 and CA 3 areas (Gasparini et al. Proc. Natl. Acad. Sci. USA 97, 9741-9746 [2000]). Neuronal nicotine acetylcholine receptors (nAChRs) activation is known to enhance neurotransmitter release. Single 
fibre whole-cell EPSCs, elicited by Schaffer collateral stimulation (double pulse protocol), were recorded in pyramidal neurons in hippocampal slices from P1-P5 old rats. Bath application of nicotine (1 $\mu \mathrm{M}$ for $3 \mathrm{~min})$ increased the probability of glutamate release converting silent synapses into functional. The percentage of successes to the first and second stimuli varied from 0 and $5.7 \%$ to 27.6 and $22.4 \%$, before and after nicotine respectively. This effect which was long lasting (for up to 2 hours) was prevented by the nicotine receptor antagonist $\mathrm{DH} \beta E(50 \mu \mathrm{M})$. This effect was mimicked by activation of $\mathrm{nAChRs}$ following stimulation of cholinergic fibers, in the presence of atropine $(1 \mu \mathrm{M})$ to block muscarinic receptors. This mechanism which is relevant for early LTP may be crucial for consolidating synaptic contacts and for rewiring immature hippocampal circuitry.

Keywords: EPSC; nicotine; cholinergic pathway; glutamate release

Green tea polyphenol (-)-epigallocatechin-3-gallate regulates secretion of non-amyloidogenic precursor protein via protein kinase c pathway in vitro and in vivo Mandel S.,_Levites Y., Amit T., and Youdim MBH. Eve Topf and US National Parkinson's Foundation Centers for Neurodegenerative diseases, Bruce Rappaport Family Research Institute, Technion - Faculty of Medicine, Haifa We have recently reported that both green tea extract, as well as its main polyphenol constituent, (-)-epigallocatechin-3-gallate (EGCG) possess potent neuroprotective activity in cell culture and mice model of Parkinson's disease. Here we show that EGCG is not only able to protect, but even to rescue rat pheochromocytoma PC12 cells against the $\beta$-amyloid (A $\beta$ ) toxicity. In addition, EGCG significantly increased ( 8 -fold) the secretion of the non-amyloidogenic soluble form of the amyloid precursor protein (SAPPa) into the conditioned media of SH-SY5Y human neuroblastoma and rat pheochromocytoma PC12 cells. The increase was dose-dependent and was blocked by the hydroxamic acid-based metalloprotease inhibitor, Ro31-9790, suggesting that the effect is mediated via $\alpha$-secretase activity. Inhibition of protein kinase $\mathrm{C}$ (PKC) with the inhibitor GF109203X, or by down-regulation of PKC, blocked the EGCG-induced increase in sAPP $\alpha$ secretion, indicating the involvement of PKC in the stimulated effect. Indeed, EGCG induced the phosphorylation of PKC, thus identifying a novel mechanism of EGCG action by stimulation of the non-amyloidogenic pathway via PKC activation. In addition, the administration of EGCG $(2 \mathrm{mg} / \mathrm{kg})$ to mice, for 7 or 14 days resulted in a significant decrease in membrane-bound holoprotein APP levels, with a concomitant increase in SAPP levels in the hippocampus. Consistently, EGCG markedly increased PKC $\alpha$ and PKC $\varepsilon$ in the membrane and the cytosolic fractions of mice hippocampus. These findings strongly suggest that EGCG has protective effects against $A \beta$-induced neurotoxicity and regulates secretory processes of non-amyloidogenic APP via PKC pathway.

Keywords: EGCG; sAPP $\alpha$; PKC; Alzheimer's disease

Neuroprotection and cell survival/cell cycle gene expression by green tea polyphenol (-)-epigallocatechin-3-gallate: involvement of protein kinase $C$ activation

Mandel S., Levites Y., Amit T. and Youdim MBH. Eve Topf and USA National Parkinson Foundation Centers of Excellence for Neurodegenerative Diseases Research,

Technion-Faculty of Medicine, Haifa

Studies from our laboratory have demonstrated that the major green tea polyphenol, (-)-epigallocatechin-3-gallate (EGCG) exerts potent neuroprotective actions against a variety of neurotoxins, both in mice and in cell culture models of Parkinson's disease (PD). Several lines of evidence correlate neuroprotection and cell survival processes with stimulation of protein kinase $\mathrm{C}$ (PKC) and extracellular signal-regulated kinases $($ ERK1/2). In this context, we tested the effects of low neuroprotective concentrations of EGCG $(0.1-10 \mu \mathrm{M})$ on these signaling pathways, employing the 6-hydroxydopamine (6-OHDA) model of PD. The present study shows a novel protective mechanism of EGCG, via restoration of the reduced PKC and ERK1/2 activities, caused by 6-OHDA toxicity, in the human neuroblastoma (NB) SH-SY5Y cell line. Since EGCG was also shown to increase phosphorylated PKC, we suggest that PKC may be necessary for its neuroprotective action against 6-OHDA. This was confirmed by the selective sensitivity of EGCG action to the inhibitor of PKC,
GF109203X $(1 \mu \mathrm{M})$. In addition, gene expression analysis revealed that EGCG prevented the 6-OHDA-induced expression of several pro-apoptotic molecules such as Bax and $\mathrm{Bad}$, as well as the decreased $\mathrm{Bcl}-2, \mathrm{Bcl}-\mathrm{w}$ and $\mathrm{Bcl}-\mathrm{xL}$. These results establish a neuroprotective mechanism of EGCG action against oxidative stress-induced cell death, via stimulation of $\mathrm{PKC}$ and modulation of cell survival/apoptotic genes. Keywords: EGCG, apoptosis, neuroprotection, PKC

Exposure to acute stress blocks the induction of long term potentiation at amygdala-prefrontal cortex synapses in vivo Maroun M. and Richter-Levin G.

The Brain and Behavior Center, University of Haifa, Haifa 31905

Most studies of stress-induced synaptic plasticity impairments have focused on the hippocampus. The present study examined whether stress, which impairs hippocampal long-term potentiation (LTP), also affects LTP at amygdala-prefrontal cortex synapses in vivo. After undergoing an inescapable stress experience, rats exhibited markedly impaired LTP without having effects on baseline transmission, in contrast, unstressed control animals showed robust LTP that persisted during the recording period. Similar to stress, LTP at the amygdala-prefrontal cortex synapses was completely blocked when the competitive NMDA receptor antagonist CPP, was injected prior the tetanus. These results demonstrate that similar to the hippocampus, LTP in the amygdala-prefrontal cortex synapses is blocked after exposure to stress and the induction of LTP in this pathway is an NMDA receptor-dependent process.

The present data show the amygdala-prefrontal pathway is amenable to long lasting plastic changes, behavioral stress and administration of NMDA-receptor antagonist CPP attenuate LTP in this pathway. Taken together these data suggest that the amygdala-prefrontal cortex pathway is relevant to stress-mediated impairments in LTP, and this pathway may have an important role in emotional memory processes. Supported by a postdoctoral fellowship to M.M from the National Institute for Psychobiology in Israel

Keywords: stress, synaptic plasticity, amygdala, prefrontal cortex

The relevance of differential response to trauma in an animal model of post-traumatic stress disorder Matar M. ${ }^{1}$, Zohar J. and Cohen $\mathrm{H}$.

${ }^{\bar{T}}$ Ministry of Health Mental Health Center, Anxiety and Stress Research Unit, Faculty of Health Sciences, Ben-Gurion University of the Negev, Beer-Sheva; ${ }^{2}$ The Chaim Sheba Medical Center, and Sackler Medical School, Tel-Aviv University

Post-traumatic Stress Disorder affects $20-30 \%$ of those exposed. Clinical studies employ stringent inclusion/exclusion criteria, yet animal studies include the entire exposed population as the study population. We examined the effect of grouping prestressed rats according to severity of response on the statistical analysis of results

The effects of exposure to a cat on behavioral measures in rats were demonstrated. Response severity was assessed and used to divide the animals into "diagnostic" groups. The two extremes were studied, i.e. those clearly "maladapted" and those clearly "well-adapted", using arbitrarily selected "Cut-off Behavioral Criteria" (CBC). The middle group was discarded for reasons of clarity. The hypothalamic-pituitary-adrenal axis and heart-rate variability were subsequently analyzed for the entire exposed population and then according to the $\mathrm{CBC}$. A single ten-minute exposure to a predator caused anxiety or fear-related behaviors. However, only $25.3 \%$ of exposed rats were affected. Compared to controls and to "well-adapted" exposed rats "maladapted" rats exhibited significantly higher plasma corticosterone and ACTH concentrations, increased sympathetic activity, diminished vagal tone and increased sympathovagal balance. These differences were significantly more obvious when data were analyzed according to $C B C$ Animals respond to stress heterogeneously, resembling humans. Overlooking this heterogeneity may significantly affect the results of bio-behavioral data analysis, and animals can (and probably should) be divided into distinct groups according to their response.

Keywords: Post-traumatic Stress Disorder, animal model anxiety, stress, maladapted, well adapted 
Imaging dementia using b value q-space analyzed diffusion

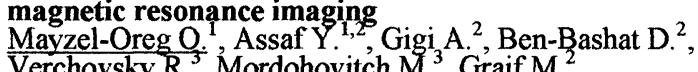
Verchovsky R. ${ }^{3}$, Mordohovitch $\mathrm{M}_{2}^{3}$, Graif $\mathrm{M}^{2}$, Rieder-Groswasser I. ${ }^{3}$, Hendler T. ${ }^{2}$, Korczyn A.D. ${ }^{3,4}$ and Cohen Y

School of Chemistry, Tel-Aviv University, Tel-Aviv 69978; ${ }^{2}$ Wohl Institute for Advanced Imaging and ${ }^{3}$ Dept. of Neurology Tel Aviv Sourasky Medical Center, Tel Aviv 64239; ${ }^{4}$ Sackler Faculty of Medicine, Tel Aviv University, Tel Aviv 69978

High b-value diffusion weighted magnetic resonance imaging is highly sensitive to white matter (WM) changes. Diffusion images, when analyzed using the q-space approach, show areas of abnormal WM in multiple sclerosis (MS) not detected by other MRI methods like $\mathrm{T}_{2^{-}}, \mathrm{T}_{1^{-}}$, weighted imaging, FLAIR, and DTI (Assaf et al, Magn. Reson. Med. 47:115-126 [2202]). When applied to vascular dementia(VaD) patients, high b-value q-space analyzed diffusion detected areas of abnormal WM extending further than $T_{2}$ apparent hyperintensities. In the areas that presented hyperintense signal in the FLAIR and $\mathrm{T}_{2}$-weighted images the WM density, as detected by q-space images, was reduced. These observations point to extensive nerve fiber loss in the $\mathrm{WM}$ in $\mathrm{VaD}$. Analysis of high $b$-value, q-space analyzed diffusion images of Alzheimer's (AD) patients also contributed information not apparent from other MRI methods. AD patients presented less marked WM density decline in the parietal lobes than $\mathrm{VaD}$ patient but similar WM loss in the frontal lobes. Interestingly, diffusion images of AD patients also showed marked effects in gray matter areas, consistent with the pathologic changes in $\mathrm{AD}$ that consist of accumulation of neurofibrillary tangles and senile plaques along with neuronal and synaptic loss leading to atrophy. To summarize, high b-value q-space diffusion MRI shows high sensitivity to WM changes and may be useful to characterize tissue loss in $\mathrm{VaD}$ and $\mathrm{AD}$, as well as to the differentiation between those two pathologies.

Keywords: Alzheimer's disease, vascular dementia, q-Space, diffusion MRI

\section{Dexanabinol and its analog PRS-211,092 inhibit}

\section{LPS-induced brain inflammation}

Meilin S., Oni E., Lavon I., Sheinin T., Avraham A., Efroni G. Lavie V., Greenberg O., Elgamiel R., Bar-Joseph A. and Fink G Pharmacology, Pharmos Ltd Kiryat Weizmann, Rehovot 76326 Dexanabinol, a nonpsychotropic cannabinoid, and its novel analog, PRS-211,092, are neuroprotective in brain ischemia induced by middle cerebral artery occlusion. Both compounds were previously shown to reduce PGE-2 secretion in LPS activated macrophages in vitro. The aim of the present study was to assess whether Dexanabinol and PRS-211,092 inhibit central neuroinflammation. C57BL mice were injected intra-cerebro-ventricularly (i.c.v) with either $250 \mathrm{ng}$ LPS (dissolved in $5 \mu \mathrm{l}$ PBS) or $5 \mu \mathrm{l}$ PBS as sham. The mice were immediately injected IP with either cremophor-ethanol (vehicle) alone, Dexanabinol $(20 \mathrm{mg} / \mathrm{kg})$ or PRS-211,092 $(20 \mathrm{mg} / \mathrm{kg})$. The mice were killed under anesthesia 24 hours and 3 days after LPS injection, and the brains removed. IL-1 beta gene expression was determined in brains extracts from animals killed at 24 hours using RT-PCR. Analysis of activated microglia was performed after 3 days using PRS-211,092 reduced the number of microglia immunoreactive cells surrounding the hippocampus by $40 \%$ and $47 \%$ respectively compared with that in animals treated with vehicle alone. IL-1 $\beta$ mRNA level was reduced by $50 \%$ in the brains of mice treated with PRS-211,092. Similar results were obtained in LPS induced mouse macrophages (RAW 264.7). These results show that Dexanabinol and PRS-211,092 can inhibit CNS microglial activation as well as IL1 $\beta$ gene expression, properties that may play an important role in the neuroprotective effect of these compounds in ischemia and brain injury.

Keywords: inflammation, gliosis, gene expression

Interaction of syntaxin $1 A$ and SNAP-25 with the C-terminus of the voltage-gated potassium channel Kv2.1 underlies their functional effects on the Kv2.1 current Michaelevski I. and Lotan I.

Dept. of Physiology and Pharmacology, Sackler School of Medicine, Tel-Aviv University

The voltage-gated potassium channel, Kv2.1, showing a delayed rectifying-type of current, exhibiting slow inactivation, is widely distributed in neuroendocrine and endocrine cells Previously we showed that Kv2.1 physically interacts with SNARE proteins, particularly, with syntaxin $1 \mathrm{~A}$ and SNAP-25. We also described the functional implication of the interaction in Xenopus oocytes. Here we present further investigations that attempt to elucidate the structural basis of such interactions. We found that nearly complete deletion of the C-terminus of Kv2.1 channel (delC416) abolished Syntaxin 1A induced left shifts of the channel steady state activation and inactivation curves. It also abolished SNAP-25 induced increase of the sustained current and right shift of the steady-state inactivation The slowing down of the onset of inactivation by SNAP-25 disappeared too. Similarly to delC416, deletion of the last two-thirds of the C-terminus (delC351) abolished the effects of syntaxin on the channel, but in contrast to delC416, only partially affected the effects of SNAP-25, diminishing the increase in the sustained current without significant effect on the steady-state inactivation curve. Based on these data we suggest that: i) syntaxin $1 \mathrm{~A}$ interacts with the proximal half of the C-terminus; ii) SNAP-25 has two sites of interaction, one, high affinity, at the proximal half, deletion of which cancels the right shift of the steady-state inactivation, and another site, low affinity, at the distal half of the channel, responsible, mainly, for the sustained current effect. These data concur with in vitro binding assays obtained in our lab (see the abstract of $S$. Tsuk). Keywords: Kv channels, syntaxin 1A, SNAP-25, C-terminus, SNARE complex

\section{Fusion of speech elements: Early auditory cortex} involvement

Mittelman N., Bleich N., Laufer I. and Pratt H. Evoked Potentials Laboratory, Technion, Haifa

Purpose: To define early auditory cortex activity to fusion of speech elements, and suggest plausible speech processing stages.

Methods: Stimuli were binaural formant transition and base, that were presented separately or fused to form the vowel-consonant-vowel sequence /ada/. Eleven right-handed, adult native Hebrew speakers listened to $2 / \mathrm{sec}$ presentations. Brain potentials from $\mathrm{Cz}$ during the $250 \mathrm{msec}$ following transition onset in the responses to transition, to the fused word and to base alone were recorded. The net-fusion response was extracted by subtracting the sum of potentials to the base and the formant transition from the potentials to the fused sound Results: Auditory middle-latency components $(20-45 \mathrm{msec})$ comprising of nine peaks and troughs were recorded in response to the base, to the formant transition and to the fused /ada/. In general, the responses to the fused object were significantly smaller in peak amplitude and in total activity (area under the curve) resulting in the difference waveform of the net-fusion response that also included 9 peaks, but with opposite polarities.

Conclusion: auditory cortex is involved in the definition and clustering of sounds elements as speech, as early as $30 \mathrm{msec}$ after stimulus onset. This early processing involves both inhibition and occlusion, and precedes the later stages of discrimination and meaning analysis.

Keywords: auditory object, streaming, cortex

\section{In vivo two-photon imaging of dendritic stability in} identified glomeruli of the mouse olfactory bulb Mizrahi A. and Katz L.C.

Howard Hughes Medical Institute and the Dept. of

Neurobiology, Duke University Medical Center, Durham, NC 27710, USA

Ongoing sensory neuron and granule cell neurogenesis results in turnover of both excitatory and inhibitory inputs to mitral cells, the main output neurons of the mammalian olfactory bulb. To evaluate the long-term stability of mitral cell dendrites in the face of these ongoing changes, we used two-photon microscopy to image mitral cell dendrites over prolonged periods in adult mice in vivo. Using transgenic mice expressing Yellow Fluorescent Protein in roughly $1 / 3$ of the mitral cell population (Feng et al., Neuron 28:41-51 [2000]), we imaged the same dendritic trees in identified glomeruli over intervals of up to two weeks. We first examined the stability of mitral cell dendrites over short and long periods. Randomly chosen areas of the dendritic arbors were reconstructed in three dimensions and compared at different time intervals. The overall arborization of dendritic trees and even the smallest distal dendritic segments remained extremely stable over periods of hours, days and weeks (imaging intervals: 1 hour $n=3 ; 24$ hours, $n=6 ; 2$ weeks, $n=3$ ). We next used intrinsic signal imaging of the dorsal surface of the olfactory bulb to identify individual glomeruli activated by a specific odorant. The sizes of glomeruli stimulated by $0.1 \%$ butanal $(n=5)$ were almost identical to the glomerular size imaged by two-photon microscopy. Given that dendritic stability of mitral cells serves as a solid baseline in control mice, we are currently using this combined approach to assess the effects of odor exposure and odor-based learning on morphological stability in identified glomeruli. 
Keywords: olfactory bulb, dendrites, two-photon microscopy, transgenic mice

\section{Pathogenic self-antigens in autoimmune disease are} potentially protective: $A$ lesson from the visual system Mizrahi T. and Schwartz M.

Dept. of Neurobiology, Weizmann Institute of Science, Rehovot 76100

Axonal injury in the central nervous system (CNS) leads to primary and secondary degeneration of the fibers, with resulting death of the corresponding cell bodies. Our laboratory recently discovered that CNS myelinated axons, after suffering a mechanical insult such as a crush injury, can benefit from the activity of autoreactive $T$ cells directed against myelin antigens (self-antigens associated with an autoimmune disease in the brain and spinal cord). Using a rat model of optic nerve crush or glutamate toxicity, we show here that vaccination with peptides derived from either interphotoreceptor retinoid-binding protein or s-antigen (a retinal self-antigens that can cause experimental autoimmune uveitinitis) reduces retinal ganglion cells (RGCs) loss resulting from either glutamate toxicity or axonal injury. In the case of glutamate insult, no such protection was obtained by vaccination with myelin antigens. These results suggest that as in the case of myelin-associated antigens, the protective antigen for RGCs is identical to the self-pathogen associated with the common autoimmune disease in this tissue (i.e. uveitis). Based on our results we propose a more general phenomenon: pathogenic-self antigens in autoimmune disease may represent the potential protective autoimmune-evoking antigens. Keywords: autoimmunity, neuroimmunology, vaccination, glaucoma, optic neuropathy

Functional properties of the anterior calcarine cortex in the human

Mukamel R. ${ }^{1}$, Levy $\mathrm{I}_{1}{ }^{1,4}$, Avidan G. ${ }^{1,4,5}$, Amedi A. ${ }^{4,5}$, Hendler T, ${ }^{2,3}$ and Malach R. ${ }^{1}$

${ }^{I}$ Dept. of Neurobiology, Weizmann Institute of Science, Rehovot 76100: ${ }^{2}$ Functional Brain Imaging Laboratory, Wohl Institute for Advanced Imaging. Tel-Aviv Sourasky Medical

Center, Tel Aviv 64239; ${ }^{3}$ Faculty of Medicine, Tel Aviv University, Tel-Aviv 69978; ${ }^{4}$ The Interdisciplinary Center for Neural Computation, Hebrew University of Jerusalem Jerusalem 91904; 'Dept. of Neurobiology, Hebrew University of Jerusalem, Jerusalem 91904

Retinotopic mapping of the human visual cortex has provided detailed information on the sequence of visual areas located ventrally and dorsally to area V1. However, little information is available concerning the functional properties of extra-striate cortex situated anterior to area V1, along the medial wall of the occipital lobe. Here we used Functional Magnetic Resonance Imaging (fMRI) on 15 different subjects, in 5 experiments in an attempt to define the functional profile of a region we term Anterior-Calcarine (AC) cortex. Ac cortex is situated anterior to area V1, just posterior to the parieto-occipital sulcus, along the ventral and dorsal banks of the calcarine sulcus. This region manifested positive fMRI signals in certain experimental conditions and negative fMRI signals in others. Conditions in which the AC had positive AMRI signals included: peripheral visual field stimulation, preferential activation to images of building compared to images of faces, and a preference to real-life photographs compared to line-drawings. Negative (inactivation) signals were found for foveal/mid eccentricity visual stimulation, and auditory stimulation by sounds of various objects (both man made objects and animal voices). No consistent meridian maps were observed in the AC. Finally, this region manifested a highly non-linear temporal signal summation so that a four fold increase in the frequency of presentation of visual images led only to a $1.26 \pm 0.13$ fold increase in signal strength. We hypothesize that the $\mathrm{AC}$ is a high order area involved in the representation of the peripheral, ambient environment. Supported by ISF 8009

Keywords: anterior calcarine, visual cortex

Glycogen synthase kinase (GSK)-3 in postmortem hippocampus of schizophrenic patients

Nadri C. ${ }^{1}$ Dean B. ${ }^{3}$ Belmaker RH. ${ }^{1,2}$ and Agam G. ${ }^{1}$ ${ }^{7}$ Stanley Research Center, Faculty of Health Science, and

Zlotowski Center for Nẹuroscience, Ben-Gurion University of the Negev, Beer-Sheva: ${ }^{2}$ Mental Health Center, Beer-Sheva; ${ }^{3}$ The Rebecca L. Cooper Research Laboratories at the Mental Health Research Institute of Victoria, Australia

GSK-3 is a protein kinase highly abundant in brain and involved in signal transduction cascades particularly in neurodevelopment. We have shown that in frontal cortex obtained postmortem GSK-3 $\beta$ is decreased at the level of protein ( $41 \%$ ), activity ( $45 \%$ ) and mRNA ( $34 \%$ ) in tissue from subjects with schizophrenia (postmortem brains were donated by The Stanley Medical Research Institute's Brain Collection), a finding that has been replicated by others at the protein leve (Beasley et al, 2001. Neurosci Lett 302:117-20). It has also been shown that GSK-3 $\beta$ is not reduced in occipital cortex of schizophrenic subjects suggesting that changes in this critical protein are regional specific. We are now extending these studies to a number of other regions of CNS using tissue from the Cooper Research Laboratories, Victoria, Australia. The hippocampus from 15 pairs of postmortem specimens from schizophrenic and controls subjects was studied blind to diagnosis. GSK-3 $\beta$ protein levels were measured using Western blot and GSK-3 activity by measuring the phosphorylation of a GSK-3 specific substrate phospho-CREB by $\left[\gamma-{ }^{32} \mathrm{P}\right]-\mathrm{ATP}$. These studies do not find that GSK-3 $\beta$ protein levels are altered in the hippocampus from subjects with schizophrenia although there is a trend to a reduction $(31 \%)$ in GSK-3 activity in that region. We are currently studying the frontal cortical specimens from the same subjects.

Keywords: schizophrenia, glycogen synthase kinase-3,

hippocampus, neurodevelopment

\section{Dynamics of cortical responses to tone pairs in relation to}

task difficulty

${ }^{1}$ Brain Research Unit, Low Temperature Laboratory, Helsinki University of Technology, Espoo, Finland: ${ }^{2}$ Permanent address: Interdisciplinary Center for Neural Computation,

Hebrew University, Jerusalem

When sounds are presented in pairs with intervals of $<300 \mathrm{~ms}$ the 100-ms cortical response $\mathrm{N100m}$ to the 2 nd stimulus is enhanced (Loveless et al., 1989). We studied the build-up of this enhancement effect and its relationship to behavioral performance and task difficulty. Whole-scalp magnetoencephalographic (MEG) signals were recorded from 10 subjects with a 306-channel neuromagnetometer. Sequences of 5 pairs of $50-\mathrm{ms}$ binaural tones were presented with stimulus-onset asynchronies of $150 \mathrm{~ms}$, inter-pair intervals of 1 $\mathrm{s}$ and an inter-sequence-interval of $10 \mathrm{~s}$. Subjects were asked to discriminate inter-pair frequencies under 2 conditions: easy $(1000$ vs. $1100 \mathrm{~Hz})$ and difficult $(1040$ vs. $1055 \mathrm{~Hz})$. In $50 \%$ of the pairs the tones were of the same frequency. All stimuli elicited prominent $\mathrm{N} 100 \mathrm{~m}$ responses in the auditory cortex, peaking at $105 \pm 4 \mathrm{~ms}$ to the $1 \mathrm{st}$ tone and at $155 \pm 15 \mathrm{~ms}$ to the 2 nd. The response to the 1st tone in a pair decreased by $46 \pm$ $3 \%$ from the 1 st to the following pairs. In contrast, the response to the 2 nd tone in a pair gradually increased in amplitude from the 2 nd to the 5 th pair. Consequently, the ratio of the 2 nd and 1st responses increased throughout the five pairs from $0.73 \pm$ 0.07 to $1.23 \pm 0.05(\mathrm{p}<0.02)$. Task difficulty did not affect the response amplitudes, whereas the response latencies to the 2 nd stimulus were $11 \pm 4 \mathrm{~ms}$ longer for the difficult condition across pairs and hemispheres $(\mathrm{p}<0.02)$; the difference was most prominent $(29 \mathrm{~ms})$ in the 5th pair (LH: $\mathrm{p}<0.03$; $\mathrm{RH}: \mathrm{p}<$ 0.05 ). Taken together, our findings suggest that the difficulty of behavioral discrimination is related to the dynamics of response build-up rather than to response strengths as such. Keywords: MEG, auditory evoked responses, pitch discrimination

Munc18 phosphorylation enhances vesicle pool refilling Nili $U^{1}$, Tonnen R. ${ }^{2}$ Verhage $\mathrm{M}^{2}$ and Ashery $\mathrm{U}^{1}$

${ }^{T}$ Dep. Of Neurobiochemistry, Wise Faculty of Life Sciences. Tel Aviv University, Tel-Aviv 69978; ${ }^{2}$ Molecular Neuroscience, Rudolf Magnus Institute, University of trecht Medical Center,Utrect, Netherlands

During repetitive or intense stimulation, neurosecretory cells and neurons undergo use-dependent changes in secretory strength which are partially mediated by activation of protein kinase C (PKC). However, the molecular targets of PKC and their mode of action remain elusive. In the present study we investigated the effect of Munc18 phosphorylation on catecholamine secretion from chromaffin cells by membrane capacitance measurements following flash photolysis of caged calcium. Munc18 is phosphorylated by PKC in a calcium-dependent manner and this phosphorylation inhibits its interaction with Syntaxin. By mutating the three PKC phosphorylation sites of Muncl8 we created the Munc18-3A mutant, which mimics the non-phosphorylated form of the native protein. Overexpression of Munc18-3A caused an increase in the number of fusion competent vesicles as well as an increase in vesicle recruitment under high calcium concentration, similar to the effect of wild-type Munc18 overexpression. In contrast, the response to a second stimulation was similar to control cells while Munc18 
wild-type overexpressing cells exhibited a larger response. This suggests that vesicle pool refilling is reduced in Munc18-3A overexpressing cells. As was shown recently, during the first flash stimulation, high calcium levels activate $\mathrm{PKC}$, which acts on specific targets to enhance vesicle pool refilling. The reduction in vesicle pool refilling observed in the Munc18-3A cells as compared to Munc18 wild-type cells suggests that Munc18 may be one of these targets. Application of phorbol ester demonstrated that $\mathrm{PKC}$ in Munc18-3A overexpressing cells can still enhance secretion but to a lesser degree than controls. These results indicate that phosphorylation of Munc18 in chromaffin cells potentiates vesicle recruitment after emptying of the releasable vesicle pools.

Keywords: Munc 18, PKC, secretion, membrane capacitance

\section{Distinct neural activity following an invalidly cued target in a cued attention task \\ Ofek E. and Pratt $\mathrm{H}$. \\ Evoked Potentials Laboratory, Technion - Israel Institute of \\ Technology, Haifa}

The cued attention task involves presentation of a cue stimulus, which provides information to direct attention to a target stimulus that follows, to which the subject has to respond. In the minority of cases the cue provides erroneous information about the target (invalid cues). The cue validity effect on brain response to the target was analyzed in the current study, and the brain structures involved were estimated.

In previous studies, a P3 component in response to the target was not found. Using a modified cued attention paradigm in the current study, a P3 component was evoked. The distinct neural activity pattern, during $\mathrm{P} 3$ period, was analyzed following identical target stimuli, which differed from each other only by the valence of the preceding cue - valid or invalid. LORETA t-test results of 10 subjects showed significantly higher brain activity (current density) following invalidly cued targets. This higher activation was especially salient on the background of lack of significant validity effect on P3 amplitude. Unique brain areas were activated following the invalidly- but not the validly cued targets. Those areas include attention related areas (BA), as well as emotion related areas (BA), and, surprisingly, also visual cortex.

In summary, distinct and significant activation was found, following identical stimuli, depending on whether they were preceded by invalid compared to valid cues. The additional brain activation in the invalid case may be related to emotional processes, attention shift, and spatial updating.

Keywords: Evoked Potentials, attention, P3, LORETA

Time and the Learning of a Cognitive Skill: The Spacing of Practice Sessions Across Days Improves Performance Ofen-Noy N. ${ }^{1}$, Dudai Y. ${ }^{1}$ and Karni A. ${ }^{2}$

Dept. of Neurobiology, Weizmann Institute of Science. Rehovot $76100^{2}$ Brain-Behavior Research Center, Haifa University, Mt. Carmel 31905

Skill is acquired in a gradual manner, i.e., repetitions lead to better performance. However, the effect of time intervals on the course of learning is less clearly understood. Here we show that spacing the same total amount of practice events over days significantly improves performance. Subjects were trained in a mirror-reading paradigm given 12 blocks of practice on a repeated list of 24 words. Practice was given in a single session or in four sessions, separated by an interval of either half an hour or 24 hours. The reaction time data was well fitted by power functions with respect to the number of repetitions. However, while practice given in the same day, i.e., in a single session or in sessions spaced with half-hour intervals, resulted in identical slopes, a significantly steeper slope was found for the group in which practice was spaced over 4 days. When three additional groups were given the same amount of training with spacing as described above, but with non-repeated words, the groups trained with a short or no time interval displayed a shallower slope compared with the group given spaced training over 4 days. Our results show that the number of repetitions during practice is not the only factor that determines the course of acquiring a cognitive skill; rather the spacing in time of practice events is important. These results are congruent with the notions that: a) that within a session training may be superfluous beyond a certain number of repetitions, b) that time-after-practice contributes to the processes of learning complex tasks.

Keywords: procedural learning, distributed practice, mirror reading
The metabotropic glutamate $\mathbf{G}$-protein-coupled receptor mGluR3 is voltage sensitive

Ohana L. Parnas I. and Parnas H.

Dept. of Neurobiology, Hebrew University, Jerusalem

G-protein coupled receptors (GPCRs) comprise the largest superfamily of proteins in mammalians and are involved in most signal transduction processes as well as in regulation of many fundamental processes such as release of neurotransmitter from nerve terminals. In spite of GPCRs being transmembrane proteins they are not considered, unlike voltage gated channels, to be voltage sensitive. However, it was shown that muscarinic receptors, GPCRs, bind ACh in a voltage dependent manner (Ilouz et al, $J$ Biol Chem, 274: 29519-29528 [1999]). Here, we examined whether mGluR3, a presynaptic autoreceptor that mediates feedback inhibition of glutamate release in CNS, exhibits voltage sensitivity. Using fresh rat brain synaptosomes we show that presynaptic glutamate receptors at their physiological environment, similarly to the muscarinic receptors, bind glutamate in a voltage dependent manner. Depolarization reduces the maximal binding of $\left[{ }^{3} \mathrm{H}\right]$ Glu several folds and further analysis revealed that depolarization reduced the fraction of the high affinity glutamate receptors. The experiments with synaptosomes could not discern whether it is the mGluR3 or the NMDA receptors (or both) that is responsible for the voltage dependent binding. This question was resolved using Xenopus oocytes. The mGluR3-mediated G-protein gated potassium channel currents were used to assay the activity of the mGluR3. We found that the apparent affinity of the mGluR3 toward glutamate was reduced upon depolarization The results presented here are compatible with the notion that the mGluR 3 is by itself voltage sensors.

Keywords: G-protein coupled receptors, metabotropic glutamate receptors, voltage sensitivity

Preparation and characterization of monoclonal antibodies directed specifically at the apolipoprotein $E$ isoform Ophir G.,Smordinsky N. and Michaelson D.M Faculty of Life Sciences, Tel Aviv University, Tel Aviv 69978

Apolipoprotein E (apoE), the most abundant lipoprotein in the brain occurs as three alleles, termed E2, E3 and E4, which differ from each other by a single amino acid substitution. Genetic and epidemiological studies revealed that the allele E4 (apoE4) is a major risk factor of chronic neurodegenerative diseases such as Alzheimer's disease (AD) and of acute insults such as head injury. The mechanisms underlying the isoform specific pathological effects of apoE4 and the molecular structural features which differentiate between apoE4 and the other apoE alleles are not known. The present study was directed at the development of $\mathrm{mAbs}$ which react isoform specifically with apoE4 in its native form and at their use together with mAbs which react equally well with the different apoE isoforms, for structural analysis of the apoE molecule. The first approach used was to immunize apoE knockout mice which express human apoE3 with purified human apoE4. This approach yielded several $\mathrm{mAbs}$ which bound specifically to apoE4 in ELISA experiments but whose binding characteristics varied in different preparations. In order to overcome this problem we undertook an alternative approach in which control mice were injected with synthetic peptides specific to apoE4 followed by screening for mAbs which bind specifically to apoE4 in immunoblot, ELISA and immunohistochemical assays and which specifically immunoprecipitate apoE4. The apoE4 specific and pan apoE mAbs thus obtained will be described and their application for structural and functional studies of apoE4 will be discussed.

Keywords: apolipoprotein E, monoclonal antibodies,

Alzheimer's disease, neurodegeneration

Critical calpain-dependent ultrastructural alterations underlie the transformation of an axonal segment into a growth cone after axotomy of cultured Aplysia neurons Oren R., Dormann A., Gitler D. and Spira M.E.

Dept. of Neurobiology, Institute of Life Science, The Hebrew University of Jerusalem

The transformation of a stable axonal segment into a motile growth cone is a critical step in the regeneration of amputated axons. In earlier studies we found that axotomy of cultured Aplysia neurons leads to a transient and local elevation of the free intracellular $\mathrm{Ca} 2+$ concentration, calpain activation localized proteolysis of the submembrane spectrin and growth cone formation. Inhibition of calpain by calpeptin prior to axotomy inhibits growth cone formation. Here we investigated the mechanisms by which calpain activation participates in the transformation of an axonal segment into a growth cone. To that end we compared the ultrastructural alterations induced by 
axotomy of cultured Aplysia neurons performed under control conditions and in the presence of calpeptin. We identified critical calpain-dependent cytoarchitectural alterations that underlie the formation of a growth cone after axotomy. Calpain-dependent processes lead to restructuring of the neurofilaments and microtubules to form a specialized cytoskeletal compartment $15-50-\mu \mathrm{m}$ proximal to the tip of the transected axon. This compartment "traps" transported vesicles and serves as a locus for microtubule polymerization. As a result, a dense pool of vesicles accumulates in close proximity to a segment of the plasma membrane along which the spectrin membrane skeleton was proteolyzed by calpain. We propose that this facilitates the fusion of vesicles with the plasma membrane, promoting the extension of the growth cone's lamellipodium. The growth process is further supported by the radial polymerization of microtubules from the growth cone's center.

Keywords: axotomy, growth cone, microtubules calpain, regeneration

\section{Opposing effects apoE3 and apoE4 on APP metabolism} following closed head injury

Oron L. ${ }^{1}$ Moskovitch L. ${ }^{1}$, Ezra Y. ${ }^{1}$, Shohami E. ${ }^{2}$ and Michaelson D.M.

${ }^{I}$ Faculty of Life Sciences, Tel Aviv University, Tel Aviv, ${ }^{2}$ School of Pharmacy, The Hebrew University of Jerusalem, Jerusalem 91120

This study examined the possibility that the pathological effects of apoE4 and the beneficial effects of apoE3 following Closed Head Injury (CHI) are mediated by interactions with the amyloid precursor protein APP. Measurements of APP and of its soluble fragment APPs in cortical homogenates revealed that they were the same in sham treated control, apoE-deficient, and apoE3 and apoE4 transgenic mice. $\mathrm{CHI}$ induced marked elevation in cortical APP and APPs levels of the apoE3 transgenic mice whereas those of the other mice groups were not significantly affected by this treatment. Furthermore the elevation in APPs in the head injured apoE3 mice was associated with elevation of APPs $\alpha$. CHI also increased the levels of cortical apoE. There were however no differences in the cortical apoE levels of the apoE3 and apoE4 transgenic mice either prior to or following head injury. Measurements of the hippocampal APP and APPs levels of sham treated mice revealed that they were the same in the different mice groups. However, $\mathrm{CHI}$ induced a significant elevation in the hippocampal APPs and APPs $\alpha$ levels of the apoE3 transgenic mice and a decrease in the corresponding APPs and APPS $\alpha$ levels of the head injured apoE4 transgenic mice. In contrast the hippocampal APP levels were not affected by CHI. These findings show that APP metabolism is affected by the apoE genotype in a brain area specific manner. These effects may play a role in the pathological effects of apoE4 and the beneficial effects of apoE3 following brain injury. Keywords: apolipoprotein E, amyloid, Alzheimer's disease, transgenic mice

\section{$\mathrm{CB}_{1}$ cannabinoid receptors play crucial role in the} development of secondary brain injury

Panikashvili D. ${ }^{1}{ }^{2}$, Mechoulam R. ${ }^{2}$ and Shohami $E^{1}$ Depts..of Pharmacology and Medicinal Chemistry ${ }^{2}$, School of Pharmacy, Hebrew University of Jerusalem.

Background: Cannabinoids, acting through specific receptors $\left(\mathrm{CB}_{1}\right.$ and $\left.\mathrm{CB}_{2}\right)$ are implicated in the control of neurological and endocrine functions. $2-\mathrm{AG}$, an endogenous cannabinoid was shown to be neuroprotective after closed head injury (CHI) [Panikashvili et al. Nature 413: 527-531, 2001]. This study examines the role of $\mathrm{CB} 1$ receptors in the development of secondary brain injury.

Methods: CB1 knockout (CB1-/-) mice and their genetic background control $\mathrm{C} 57 \mathrm{BL} / 6 \mathrm{~J}$ were used. $\mathrm{CHI}$ was induced on the exposed skull of mice under ether anesthesia using weight-drop device. Edema was evaluated $24 \mathrm{~h}$ after $\mathrm{CHI}$ using the wet:dry weight ratio. The neurological status was evaluated using a 10-points scoring system (neurological severity score, NSS). $\triangle$ NSS reflects recovery after $\mathrm{CHI}$ and is calculated at any time $(\mathrm{t})$ as the difference $\mathrm{NSS}(1 \mathrm{~h})-\mathrm{NSS}(\mathrm{t})$. 2-AG, dissolved in ethanol:emulphor:saline (1:1:18) was injected subcutaneously immediately after injury.

Results: Interestingly, $\mathrm{CBl}(-/-)$ mice suffered some (non-significant) basal clinical impairment, as shown by the pre-injury NSS $(1.57 \pm 0.3$ vs. $0.56 \pm 0.3, p=0.08)$. These mice showed almost no clinical recovery at $24 \mathrm{~h}$ after $\mathrm{CHI}$, in contrast to the controls $(\Delta \mathrm{NSS}=0.71 \pm 0.29$ vs. $1.89 \pm 0.26$, $\mathrm{p}=0.016)$. To further demonstrate the role of $\mathrm{CB1}$ in recovery after $\mathrm{CHI}, 2-\mathrm{AG}$ was injected to $\mathrm{CB} 1(-/-)$ mice. This treatment, which was effective in the controls, had no effect on the neurological recovery of $\mathrm{CB} 1(-/-)$ mice $(\triangle \mathrm{NSS}=2.86 \pm 0.55$ vs $0.43 \pm 0.3$ respectively, $p=0.0041$ ) and did not reduce edema $(78.81 \pm 0.34$ vs. $80.57 \pm 0.61 \%$ water, $p=0.0308)$. These findings strongly indicate that $\mathrm{CBI}$ receptors are involved in the attenuation of secondary damage after $\mathrm{CHI}$

Keywords: traumatic brain injury, 2-arachidonoyl-glycerol (2-AG), Cannabinoids (CB), CB1-receptor, neuroprotection

Inter-hemispheric perceptual learning transfer depends on task difficulty

Pavlovskaya M. ${ }^{1}$ and Hochstein $\mathrm{S}^{2}$

'Loewenstein Rehabilitation Hospital, Raanana, 'Sackler School of Medicine, Tel-Aviv University, Tel Aviv 69978; '2ife Sciences Institute \& Neural Computation Center, Hebrew University, Jerusalem; ${ }^{1,2}$ Smith Psychobiology Institute, Jerusalem

We previously found hemispheric differences in feature search (Pavlovskaya et al, Spatial Vision 14:151-73, 2001) when search arrays were in one or the other hemifield, rather than when the array was central and target elements lateral central In parallel, Ahissar \& Hochstein (Nature, 387, 401-6, 1997) found perceptual learning transfer across position or orientation depends on task condition: Learning effects transfer when the task is easy (large target-distractor difference; limited target position uncertainty; long test-to-mask delay) and are considerably specific when conditions are harder. These easy vs. hard condition differences were related to cerebral sites of training modification: hard tasks were seen as requiring low-level (specific) representations while easy tasks are performed using high cortical level mechanisms alone. We ask if inter-hemispheric transfer also depends on task difficulty, since high-level receptive fields include contra-lateral areas Subjects performed color and orientation feature search, each within one hemifield. Sessions were half easy, half hard Following training, we switched the sides of color and orientation tasks, and found nearly complete transfer for easy conditions, and considerably less with difficult conditions. Our results support the conclusion that easy search depends on high cortical level mechanisms, while hard search is performed by guided return to low levels when fine discriminations are required. Practice improves performance by modifying high or low levels, accordingly. Thus, easy task performance and learning take place at a cortical area sufficiently high that its receptive fields are not limited to a single hemifield.

Keywords: visual search, perceptual learning, inter-hemispheric transfer

\section{Neuroscience the science for psychiatry}

Peled A.

Institute for Psychiatric Studies, Sha'ar Menashe Mental

Health Center \& Bruce Rappaport Faculty of Medicine,

Technion, Israel Institute of Technology, Haifa

Psychiatry was the only medical discipline that had no scientific foundation until recent years. Biological psychiatry began approximately half a century ago when medications were discovered. The so called "biological" effect of medication on mood affect and behavior initialized an enthusiastic surge of biological investigations in psychiatry. Research was directed to biochemistry and thus findings are confined to neurotransmitter and receptor alterations relevant to medications and their clinical effects. This trend of biological psychiatry is at its peak even nowadays. To be a psychiatrist with molecular biology education would advance your career enormously. Disappointing however are the advances for the patients. Even though patients' symptoms have improved, patients are not anywhere near real cure conditions, especially if serious mental disorders such as schizophrenia are targeted. Both the molecular biology as well as the genetic research findings failed to approach the big picture of mental disorders as brain diseases. To put the findings of biological psychiatry in any useful context the brain as a dynamic organization of neural networks has to be addressed. Cutting-edge thinking about mental disorders poin to neuroscience as the future science for psychiatry. Mental disorders are thus disturbances in the organization of complex dynamic interacting cell ensembles and ever-changing neurona networks spread in the brain. The future of psychiatry will require understanding of complex non-linear dynamics. Concepts such as criticality and optimizations, that are currently alien to psychiatrists will be the basis of understanding mental conditions. Instead of horrifying nomenclatures such as "schizophrenia" in an ineffective low-reliability diagnostic system, futuristic psychiatry will diagnose "optimization breakdowns" in the brains of patients Psychiatric treatment will be directed toward re-optimization of neural brain systems for real cure of mental illness. 
Keywords: psychiatry, complexity, system theory, functional connectivity

\section{Observation of spontaneous cortical layer activity using} physiological MRI noise

Pelled G. and Goelman G.

HBRC, medical biophysics and Nuclear Medicine Dept.,

Hadassah Hebrew University Hospital, Jerusalem

Measuring the functional magnetic resonance imaging (fMRI) blood-oxygenation level dependent (BOLD) signal non-invasively from the different layers of the cortex, can expand our understanding to the current debate in the fMRI community regarding the neuronal source of the fMRI signal, since the cortex is composed of six layers that different in there cell bodies, synapses and dendrites density. Using MRI physiological noise we measure the BOLD fluctuation of the cortical layers during rest. As a model we use the rat's visual cortex, which has been extensively studied using electrophysiology methods. Two different statistical approaches were used. In the first, for each voxel temporal SD were calculated and averaged together. In the second, the area spatial SD were calculated and averaged along time. Generally, the first approach measures the level of spontaneous neuronal activity, while the second approach measures the homogeneity behavior of the neuronal compartments. Our results show high temporal fluctuations in the deeper layers, indicating high spontaneous activity in these layers and low spatial fluctuations in these layers, indicating high homogeneity behavior. These results are in agreement with previous electrophysiology studies, and demonstrate that the physiology MRI noise is sensitive enough to detect localize neuronal activity and neuronal homogeneity behavior

Keywords: cortical layers, BOLD, fMRI

Early coassembly of KCNQ1 with KCNE1 or Yotiao but not with an LQT5 KCNE1 mutant prevents channel inhibition by a tetramerization peptide

Peretz A. ${ }^{\text {, Uziyel Y Y. }}{ }^{\text {, }}$, Schmitt N. ${ }^{2}$, Ben Aharon-Shamgar L. ${ }^{1}$, Schottelndreier $\mathrm{H}^{1}$, Pongs $\mathrm{O}^{2}$. and Attali B.

${ }^{I}$ Sackler School of Medicine, Dept. of Physiology \&

Pharmacology, Tel Aviv University; ${ }^{2}$ Institut fuer Neurale Signalverarbeitung, ZMNH, Hamburg, Germany

The $\mathrm{I}_{\mathrm{Ks}}$ potassium channel complex consists of the heteromeric assembly of two structurally distinct $\alpha$ and $\beta$ subunits called KCNQ1 and KCNE1, respectively. Mutations in KCNQ1 and KCNE1 genes produce the long QT (LQT) syndrome, a human genetic cardiovascular disease. We recently identified a small region in the KCNQ1 C-terminus (aa. 589-620) which functions as an assembly domain for KCNQ $1 \propto$ subunits. Here we show that co-expression of WT KCNQ1 with CAD, a KCNQ1 C-terminus peptide (510-620), suppresses $\mathrm{K}^{+}$channel activity. CAD inhibits KCNQ1 currents probably by inhibiting the tetrameric assembly of WT subunits as it encompasses the channel assembly domain and a $\alpha$ helix structure that is part of a calmodulin-binding domain. Confocal immunocytochemical images confirm the virtual absence of KCNQ1 expression in the plasma membrane. Co-expression of WT KCNE1 with WT KCNQ1 and CAD, fully restores the $\mathrm{K}^{+}$channel activity and the plasma membrane labeling of KCNQ1 and KCNE1 proteins. Conversely, a naturally oecurring LQT5 mutation of $\mathrm{KCNE} 1$ (W87R), located at the C-terminus, is unable to fully restore neither functional $\mathrm{K}^{+}$currents nor the membrane localization of the channel subunits. Yotiao, an adaptor protein that binds to the same KCNQ1 assembly domain could restore like other $\beta$ subunits (KCNE2 and KCNE3) functional $\mathrm{K}^{+}$ currents when co-expressed with WT KCNQ1 and CAD. In all these data suggest that the early co-assembly of KCNQ1 with KCNE1 or Yotiao, but not with an LQT5 KCNE1 mutant (W87R), prevents channel inhibition by the CAD tetramerization peptide.

Keywords: K+ channels, assembly, KCNQ

Meclofenamic acid and 1-EBIO, novel KCNQ2/3 potassium channel openers: therapeutic implications for epilepsy and neuroprotection

Peretz A., Uziyel Y. and Attali B.

Dept. of Physiology and Pharmacology, Sackler School of Medicine, Tel Aviv University, Tel Aviv 69978

Voltage-dependent $\mathrm{K}^{+}$channels play a major role in brain functions. Among them, the M potassium current has profound effects on brain excitability as its low-threshold gating and its slow activation and deactivation act as a brake for neuronal firing. Recently, the KCNQ2/3 channel complex belonging to the KCNQ family of voltage-dependent $\mathrm{K}^{+}$channels was identified as the molecular correlate of the M-current. Furthermore, the KCNQ2 and KCNQ3 channel $\alpha$ subunits are mutated in families with benign familial neonatal convulsions, a neonatal form of epilepsy. In this study, we characterize two novel openers of KCNO2/3 channels, meclofenamic acid and 1-EBIO. Using CHO cells transfected with the KCNQ2/KCNQ3 cDNAs and the patch-clamp technique, we found that meclofenamic acid and 1-EBIO activate KCNO2/3 channels, by causing a marked leftward shift in the voltage dependence of activation $(-23 \mathrm{mV}$ and $-8 \mathrm{mV}$, respectively). In addition, both compounds slow down the channel deactivation kinetics. These openers increase the KCNQ2/3 current amplitude at physiologically relevant potentials $(-70 \mathrm{mV}$ to 0 $\mathrm{mV})$. Recording of membrane potential in Xenopus oocytes expressing KCNQ2/3 channels indicates that incubation with $30 \mu \mathrm{M}$ meclofenamic acid within 1-2 min produces a hyperpolarization from a resting potential of $-62 \mathrm{mV}$ to -78 $\mathrm{mV}$. Interestingly, these openers potently and reversibly blocked the evoked and spontaneous electrical activity of rat cortical neurons. In view of the crucial role of KCNQ2 and KCNQ3 channel subunits in epilepsy and neuronal excitability, enhancement of KCNQ2/3 potassium currents by these openers may prove to be an important target for future anti-epileptic and neuroprotective therapy.

Keywords: $\mathrm{K}^{+}$channels, epilepsy, KCNQ

Visual deficits associated with object images revealed by fMRI in human amblyopis

Pianka $\mathrm{P}^{1}{ }^{1}$, Lerner Y. ${ }^{2}$, Azmon B. ${ }^{1}$, Leiba $\mathrm{H}^{3}$, Stolovitch $\mathrm{H}^{1}$, Loewenstein $A^{1}{ }^{1}$, Harel M. ${ }^{2}$, Malach $\mathrm{R}^{2}$ and Hendler T. ${ }^{\prime}$ Tel-Aviv Sourasky Medical Center, Tel Aviv: ${ }^{2}$ Weizmann Institute of Science, Rehovot 76100; ${ }^{3}$ Kaplan Hospital, Rehovot Amblyopia is a visual developmental disorder characterized by abnormal foveal vision due to aberrant early visual experience In normal subjects foveal vision is associated with the representation of faces (Levy et al. Nature Neurosci 2001) Here we explored possible relationship between the amblyopic deficit and fMRI activation to various object categories. Nine unilateral amblyopic and 3 healthy subjects with normal vision were studied. Three fMRI experiments were conducted using red-green filters for monocular stimulation: (1) Retinotopic mapping of the sound eye (2) Presentation of small and large pictures correlating to visual acuity of $6 / 6$ and $6 / 60$ respectively (3) Line drawings of faces and houses of equal size presented to each eye. In low order visual areas (e.g retinotopic) the sound eve's activation was similar to normal subjects. However, in the amblyopic eye, the activation for small pictures was markedly reduced compared to the sound eye, while large pictures' activation was only slightly reduced In high order visual areas the sound eye's activation was similar to normal subjects while the amblyopic eye showed marked activation reductions that appeared to be more emphasized in the fusiform gyrus compared to the collateral sulcus. We conclude that abnormal early visual experience resulting in amblyopia affects in a selective manner the central representation of objects both in low and high order visual cortex regions.

Supported by Adam's super-center for brain studies grant and Israel Academy grant No. 27324.

Keywords: amblyopia, visual system, fMRI

\section{Improving vision in adult amblyopia by perceptual}

${ }_{\text {Polat U. }}^{\text {learning }}$, Ma-Naim T. ${ }^{1}$, Belkin M. ${ }^{1}$, Bonneh Y. ${ }^{3}$, and Sagi D. ${ }^{3}$ ${ }^{T}$ Goldschleger Eye Research Institute, Tel-Aviv University, Sheba Medical Center. Tel Hashomer $52621:{ }^{2}$ NeuroVision. Inc., P.O. Box 1888, Ramat Gan, 52136: ${ }^{3}$ The Weizmann Institute of Science. Dept. of Neurobiology, Rehovot. 76100 Practicing certain visual tasks leads to a significant improvement in performance, a process termed "perceptual learning". Learning was shown to be specific for basic stimulus features such as local orientation, retinal location and eve of presentation, suggesting plasticity of the primary visual cortex in adults. However, it has never been shown that such a low-level learning may have impact on higher-level visual tasks, like recognition. Amblyopia is characterized by several functional abnormalities in spatial vision, including reduction in visual acuity (VA), contrast sensitivity function (CSF), and vernier acuity, as well as spatial distortion, abnormal spatial interactions and impaired contour detection. The visual deficiencies are thought to be irreparable after the first decade of life once the developmental maturation window has been terminated. The vision loss is thought to result from abnormal operation of the neuronal networks within the primary visual cortex, most notably, orientation selective neurons and their interactions. The perceptual learning procedure described here was designed to train this network by efficiently stimulating these neuronal populations and effectively promoting their 
spatial interactions. Here, using a systematic low-level training of a malfunctioning adult visual system, we show that inducing low level changes may yield significant perceptual benefits and transfer to higher visual tasks. The training procedure produced a two-fold improvement in CSF and letter recognition tasks in the treatment group $(\mathrm{N}=63)$. No improvement found in the control group $(\mathrm{N}=14)$. The results demonstrate that perceptual learning can improve basic representations within the adult visual system that did not develop during the critical period. Keywords: vision, perceptual learning, development, amblyopia, lateral interactions

The adverse effects of repeated intraperitoneal injections are reversed by chronic treatment with the antidepressant drug imipramine

Pollak Y. ${ }^{1}$ Ovadia $\mathrm{H}^{2}$ and Yirmiya $\mathrm{R}^{1}$

${ }^{7}$ Dept. of Psychology, The Hebrew University of Jerusalem, Mount Scopus, Jerusalem; ${ }^{2}$ Dept. of Neurology

Hadassah-Hebrew University Hospital, Jerusalem

Previous research suggested that repeated handling and intraperitoneal (i.p.) injections constitute a chronic stressor, producing depressive-like physiological consequences. This present study was aimed to characterize further the effects of repeated saline injections in SJL/J mice, particularly in the context of experimental autoimmune encephalomyelitis (EAE). Mice were injected daily with saline $(10 \mathrm{ml} / \mathrm{kg})$ for 3 weeks or not handled. Alterations in body weight, spleen weight and blood interleukin-1 (IL-1) protein levels were measured, as well as the susceptibility to the induction of EAE by myelin auto-antigen. Repeated injections were associated with lower body weight and higher levels of IL-1. No effect on spleen weight was recorded. Following immunization with PLP, a common myelin-associated protein, chronically injected mice presented with aggravated clinical symptoms of EAE and higher mortality rate. Chronic treatment with daily injections of the tricyclic antidepressant drug imipramine $(10 \mathrm{mg} / \mathrm{kg} /$ day $)$ imipramine, completely reversed the effects of repeated injections on body weight and EAE-associated mortality, and significantly attenuated the effects on IL-1 production and EÅE-associated symptoms severity. Oral administration of imipramine $(10 \mathrm{mg} / \mathrm{kg} /$ day dissolved in the drinking water) had no effect on non-injected EAE mice, indicating that the effects of imipramine on EAE were limited to counteracting the consequences of repeated injections. In conclusion, repeated injections in mice are associated with imipramine-suppressible adverse physiological consequences that may be related to higher production of pro-inflammatory cytokines. These results highlight the importance of control non-handled animals in any study design consisting of repeated i.p. injections

Keyword: repeated injections, stress, experimental

autoimmune encephalomyelitis, interleukin-1

Chronic treatment from adolescence with TV-3326, a brain-selective MAO-cholinesterase inhibitor, abolishes hyperanxiety in adult prenatally-stressed rats Poltyrev T. ${ }^{1}$ Youdim $\mathrm{MBH}^{2}{ }^{2}$, Derigues $\mathrm{N}^{2}$ and Weinstock $\mathrm{M}^{1}$ ${ }^{T}$ Dept. of Pharmacology School of Pharmacy, Hebrew University, Jerusalem; ${ }^{2}$ Eve Topf and NPF Centers, Technion, Haifa

Chronic treatment with tricyclic antidepressants (TAD) and monoamine oxidase (MAO) inhibitors may be as effective as benzodiazepines in the treatment of chronic anxiety states (Feighner, J. Clin. Psychiatry 60: 2218S-2222S [1999]) Studies in animals in which anxiety was induced by acute stress and was sensitive to benzodiazepines failed to demonstrate an anxiolytic effect of TADs. Chronic hyperanxiety and depressive-like behaviour can be induced in primates and rodents by prenatal stress. The aim of the presen study was to see whether amitriptyline and TV-3326, a novel MAO-cholinesterase inhibitor, had anxiolytic effects in this model. Male and female offspring [PS] of rats (at least 9/group) that had been stressed by daily restraint during gestation and of control rats [C], were given water amitriptyline $(4.5 \mathrm{mg} / \mathrm{kg} / \mathrm{day})$ or TV-3326 $(17 \mathrm{mg} / \mathrm{kg} / \mathrm{day})$ in the drinking water from the age of 6 to 12 weeks. TV-3326 inhibited MAO-A and B in the brain by $70 \%$ and $60 \%$ respectively. During the 12 th week, PS and $\mathrm{C}$ rats were tested in the elevated plus-maze, a validated test for anxiety. TV-3326 selectively increased the time spent by PS but not $C$ rats in

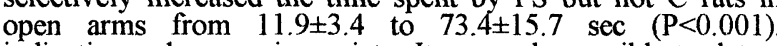
indicating a decrease in anxiety. It was only possible to detect an anxiolytic effect of amitriptyline in PS rats 2 weeks after cessation of treatment, when its depressant effect on exploration in $\mathrm{C}$ rats under continuous treatment was no longe seen. In conclusion, chronic administration of TV-3326, selectively abolishes hyper-anxiety induced by prenatal stress without suppressing behaviour of controls.

Keywords: antidepressant, chronic anxiety, prenatal stress, plus maze

Reversible internalization of voltage gated channels accompany brefeldin A-induced structural remodeling of cultured Aplysia neurons

Prager-Khoutorsky M. and Spira M.E

Life Sciences Institute, The Hebrew University of Jerusalem

Eukaryotic cells constitutively internalize the plasma membrane. Nevertheless, the morphology and membrane properties of the cells are maintained constant by compensatory constitutive exocytosis. Recently, we reported that the neurites and axons of cultured Aplysia neurons are internalized within $24-48 \mathrm{hr}$ of $5 \mu \mathrm{g} / \mathrm{ml}$ brefeldin A application (Prager-Khoutorsky and Spira 2000, Neuroscience Letters supplement 55, S43). When brefeldin is washed away, the Golgi apparatus reassembles, and the neuron extends new neurites. Here we report that the passive and active membrane properties of the neurons undergo alterations during these restructuring processes. Control experiments revealed that both the cell body and axons are excitable and that the action potentials are not blocked by BFA. We found that for as long as the axons are not totally internalized (in BFA) depolarization of the neuron evokes action potentials. Nevertheless, when the axons are internalized depolarization of the formally excitable cell body fails to generate action potentials. We thus conclude that the cell body looses it excitability during the axon's membrane internalization. Cytochalasin B inhibits the effects of BFA. Under these conditions the cell's input resistance and excitability are not altered. When BFA is washed and the naked cell body begins to extend growth cones, depolarization generates normal action potentials. These observations suggest that the newly formed vesicles carry to the plasma membrane the normal repertoire of voltage-gated channels to generate action potentials. We conclude that regulation of the balance between constitutive exocytosis and endocytosis leads to remodeling of the morphology and the biophysical properties of adult neurons.

Keywords: neuroplasticity, Golgi, endocytosis exocytosis, Aplysia

\section{The effect of external noise on word span}

Putter-Katz $\mathrm{H}^{1,2}$ and Ahissar M. ${ }^{1,2}$

${ }^{1}$ Interdisciplinary Center for Neural Computation, Hebrew University of Jerusalem; ${ }^{2}$ Dept. of Psychology. Hebrew University of Jerusalem

Previous studies documented strong correlations between auditory perception and working memory in the genera population, learning impaired subjects and hearing impaired individuals. We hypothesized that the items retained in working memory are those perceived with sufficiently high signal to noise ratio, with whole words as default candidates When signal to noise ratio is low, either due to external or to internal noise, the items retained in memory will be sub-word elements, and consequently word span will decrease dramatically. We tested this hypothesis in the general population. Subjects were asked to recall sequences of 1-6 two-syllable pseudowords that were presented in quiet and in noise. For the majority of subjects the addition of noise decreased single word repetition accuracy by $10-20 \%$. For these subjects, word span in noise was significantly lower than one would expect had span (memory) and repetition accuracy been independent. When sequences were longer than two words their repetition accuracy dropped dramatically. These results are consistent with our hypothesis that decreased perception (signal to noise ratio) affects level of representation in working memory and consequently decreases memory span Keywords: speech perception, verbal memory span, signal to noise ratio

Linking morphology and homeostatic synaptic plasticity using a CA1 pyramidal neuron model

Rabinowitch . $^{.}$, London M. ${ }^{3}$ and Segev I. ${ }^{1,2}$

Interdisciplinary Center for Neural Computation and ${ }^{2}$ Institute for Life Science. Hebrew University Jerusalem:

${ }^{3}$ University College, London

A recently discovered plasticity effect, termed "homeostatic plasticity', takes place when synaptic conductance is adjusted to maintain what seems to be a stable level of activity of the cell. This process is much slower than LTP/LTD (on the order of hours to days) and has been found to negatively correlate with the level of depolarization. In this study, a detailed model of a CAl neuron was used to explore the impact of dendritic 
morphology on synaptic homeostatic plasticity. The model consists of numerous excitatory synapses that are evenly spread on the dendrites, together with uniformly distributed active ion channels, giving rise to back-propagating action potentials. Synapses were activated by stationary stochastic inputs. A simple local rule was applied to iteratively scale synaptic conductance so as to keep the average local dendritic membrane potential at a predetermined level. The synapses progressively reached an average steady state conductance with a bell-shaped profile along the apical tree, peaking at about midway from soma to distal tufts. This is reminiscent of recent findings on the distribution of synaptic conductance in CA1 neurons. In the shorter basal tree, only the initial increase in synaptic conductance with distance from soma was apparent. We demonstrate that there is an interesting interaction between specific dendritic morphology, membrane excitability and synaptic plasticity. Together they shape local voltage perturbation as well as spatial interaction (the "region of influence") of one synapse on others; this in turn affects the global activity of the neuron (e.g., axonal spikes). In consequence, very local plastic rules result in a global cooperative effect.

Keywords: synapse, plasticity, homeostasis, morphology

Protection against soman-induced brain damage and cognitive deficits by an antidotal treatment with anticholinergic and antiglutamatergic agents Raveh L., Brandeis R., Gilat E., Cohen G., Alkalay D., Rabinovitz I. and Weissman B.A

Dept. of Pharmacology, Israel Institute for Biological

Research, P. O. Box, I9, Ness Ziona

The protective effects of post exposure antidotal treatments combined with pyridostigmine pretreatment on the deleterious consequences of soman poisoning were studied. TAB (a mixture of TMB4, atropine and benactyzine) was injected concomitant with the development of toxic signs whereas scopolamine $(0.1 \mathrm{mg} / \mathrm{kg})$ or caramiphen $(10 \mathrm{mg} / \mathrm{kg})$ were administered five min after soman $\left(1.2 L_{50}\right)$. Atropine (4 $\mathrm{mg} / \mathrm{kg}$ ) was given at onset of toxic symptoms to the latter two groups. It was found that caramiphen and TAB completely abolished electrographic seizure activity while scopolamine treatment exhibited only partial protection. Additionally, no significant alteration in the density of peripheral benzodiazepine receptors were noted ensuing the former therapies whereas scopolamine application resulted in a complex outcome: while part of the animals demonstrated no change in the number of these sites, the other exhibited markedly higher densities. Cognitive functions (learning and memory processes, evaluated using the Morris Water Maze) were improved by the three treatments compared to soman-injected animals, with the following rank order: caramiphen $>\mathrm{TAB}>$ scopolamine. Statistically significant correlations were demonstrated between learning parameters and $\left[{ }^{3} \mathrm{H}\right] \mathrm{Ro5}-4864$ binding to forebrain/midbrain membrane preparations $(r=0.72 ; r=0.73)$. These results show that caramiphen and $\mathrm{TAB}$ have a considerable potential as post exposure therapies against soman intoxication.

Keywords: soman, Morris Water Maze, PBR, protection

What is the function of the "visual" cortex in the blind? Raz N. ${ }^{1}$, Amedi A. ${ }^{1}$ Pianka P. ${ }^{2}$, Hendler T. ${ }^{2}$, Malach R. ${ }^{3}$, Ahissar ${ }^{1}{ }^{1}$ and Zohary E.

${ }^{1}$ Hebrew University, Jerusalem 91904; ${ }^{2}$ Sourasky Medical Center, Tel Aviv 64239; ${ }^{3}$ Weizmann Institute of Science,

\section{Rehovot 76100}

The areas devoted to vision in the primate brain constitute $\sim 25 \%$ of the cortical sheet. Does loss of vision render these regions useless. Recently, using fMRI mapping, we showed that the occipital cortex of the congenitally blind is activated during various high level cognitive tasks, including auditory verb generation and verbal memory. This activation was found in all blind subjects $(\mathrm{N}=10)$, in regions corresponding to retinotopic visual areas in sighted subjects. If the occipital cortex of the blind has any functional role, one might expect the level of occipital activation to be correlated with the subject's performance in similar tasks. We therefore measured the subjects' verbal and memory skills, using the standard Wechsler tests. Interestingly the blind as a group were far superior in their memory performance compared to the sighted controls (for example digit span- blind: 14.3 \pm 1.36 SEM compared with the grand population norm of 10). Second, the extent of fMRI activation and its significance level differed substantially between individual subjects. Crucially, strong positive correlation ( $r \sim 0.8-0.9)$ was also observed between the individual subjects' fMRI signal during verbal memory recall and their performance in the Wechsler's memory test. These results suggest that the occipital cortex of the congenitally blind may undergo a radical change to be seemingly involved in language and memory functions.

Supported by Israel Science Foundation grant number 8009

Ethyl-substituted carbamates show much lower acetylcholinesterase inhibitory potency than those with smaller or larger n-alkyl substituents

Razin M. ${ }^{1}$, Sterling J. ${ }^{2}$, Herzig Y. ${ }^{2}$, Weinstock M. ${ }^{1}$

${ }^{T}$ Dept. of Pharmacology, Hebrew University, Jerusalem ${ }^{2}$ Medicinal Chemistry R\&D Department, Teva Pharmaceutical Industries, Netanya

The only clinically proven therapeutic approach for the treatment of Alzheimer's disease is inhibition of acetylcholinesterase (AChE) which includes alkylcarbamates, such as physostigmine, rivastigmine and heptylphysostigmine. Several researchers have noted the surprisingly low potency of ethyl-substituted carbamates compared with methyl substituents, but there have been very few studies on AChE inhibitory activity of longer chain alkyl substituents. The aim of the present study was to measure the kinetics of AChE inhibition ( $k$ i) at $37^{\circ} \mathrm{C}$ in different homologous series of carbamates with an $\mathrm{H}$ or methyl group as one substituent $\left(\mathrm{R}_{1}\right)$ and a methyl, ethyl, n-propyl, n-butyl, n-hexyl or methoxyphenyl as the other $\left(R_{2}\right)$, using human erythrocyte $\mathrm{AChE}$, according to the scheme:

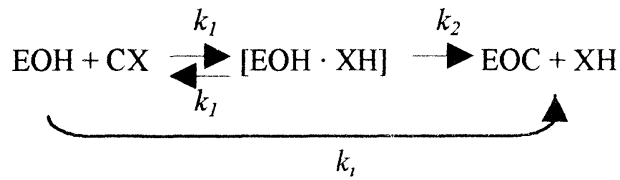

EOH, enzyme; $C X$, intact carbamate; EOC, carbamylated enzyme; ki, apparent bimolecular rate constant governing overall inhibition rate

Our results showed that $k i$ was highest when $R_{1}$ and $R_{2}$ were methyl in all series, (e.g. $4081 \quad\left[\mathrm{M}^{-1} \mathrm{~min}^{-1}\right]$ in the 6-carbamoylaminoindan series). This was reduced to 102 $\mathrm{M}^{-1} \min ^{-1}$ when $R_{2}$ was ethyl and gradually rose with increasing chain length to $3055 \mathrm{M}^{-1} \mathrm{~min}^{-1}$ for $\mathrm{n}$-hexyl and $7111 \mathrm{M}^{-1} \mathrm{~min}^{-9}$ for methoxyphenyl. A very similar relationship was found for carbamate derivatives of 3-hydroxyphenethylamines.

The high potency of the aromatic carbamates may be explained by $\pi-\pi$ interactions with phenylalanine residues near the active site of AChE, however, the unusual SAR of the alkyl substituents is more difficult to justify and may involve interactions at the peripheral anionic site (Lin et al, Bioorganic \& Medicinal Chem . 7:2683-2689 [1999]).

Keywords: acetylcholinesterase inhibition, carbamates, structure-function relationship

\section{LIS1: from brain to cell}

Reiner O., Caspi M., Gdalyahu A., Ghosh I. and Levy T. Dept. of Molecular Genetics, Weizmann Institute of Science

Formation of the brain structure in human is a complex process that occurs during several months of prenatal development One of the most striking features of the human brain is its characteristic convolutions. These convolutions are lacking in a severe human brain malformation known as lissencephaly (lissos $=$ smooth , encephaly = brain). About one in 30,000 life births are affected with this disease. Lissencephaly patients have a reduced life expectancy and a severe mental retardation. So far, two genes have been found to be mutated in lissencephaly; IISI located on chromosome 17 and Doublecortin an X-linked gene. LIS1 is a WD repeat protein and is known to be involved in several protein complexes and microtubule regulation by direct interactions with tubulin or via interactions with the dynein/dynactin motor complex. We describe the phenotype observed in Lis 1 mutant mice in the context of LIS 1 protein interactions. In addition, we discuss an interaction with the cytoplasmic linker protein CLIP-170 CLIP-170 is required for in vitro binding of endocytic transport vesicles to microtubules. We suggest that CLIP-170 and LIS1 are both required for cargo transport via the dynein motor complex. Using the knowledge we gained regarding LIS1 function we have analyzed a set of LIS1 point mutations that result in variable lissencephaly-related phenotypes. Our detailed protein function analysis allows to understand the spectrum of the disease phenotype.

Supported by HFSP grant No: RG283199 9. March of Dimes grant No: 6-FY01-5. ISF grant 19/00 and Minerva Foundation Keywords: LIS 1, microtubule, lissencephaly 
The effect of cytotoxic lesions of the hippocampus on spatial memory in GluR1-/- mice Reisel D. ${ }^{\text {; }}$ Bannerman D.M. ${ }^{1}$, Schmitt W. ${ }^{1}$; Deacon R.M.J. ${ }^{1}$ Flint J.'; Borchardt T. ${ }^{3}$, Seeburg P.H. ${ }^{3}$; Rawlins J.N.P. ${ }^{1}$ ${ }^{1}$ Dept. of Experimental Psychology, University of Oxford, Oxford, U.K.; ${ }^{2}$ Wellcome Trust Centre for Human Genetics, Oxford, U.K.'; ${ }^{3}$ Dept. of Molecular Neurobiology, Max-Planck Institute of Medical Research, Heidelberg, Germany

L-alpha-amino-3-hydroxy-5-methylisoxazole-4-propionate (AMPA) receptor activity is required for long-term potentiation (LTP), a postulated model of cellular mechanisms underlying learning and memory. GluR1 (GluR-A), one of four AMPA receptor subtypes, is thought to mediate a postsynaptic mechanism of LTP expression. Previous studies have demonstrated that GluR1-deficient mice lack LTP in the afferent pathways to the CAl area of the hippocampus, yet their spatial reference memory is intact (Zamanillo, D. et al. 1999. Science 284: 1805-1811). One way of explaining the intact learning ability in the GluR1-/- mice is that they employ extra-hippocampal structures to solve the spatial learning tasks. We tested this hypothesis by cytotoxic lesion of the hippocampus using an injection of NMDA. The mice were then tested in the standard version of the Morris water maze task and on a reference memory task on the elevated Y-maze. Performance in the water maze and on the Y-maze was impaired by lesion but not by GluR1 deletion. This suggests that extra-hippocampal compensation cannot account for the spared ability of the GluR1-\% mice in spatial learning tasks such as the Morris water maze and the Y-maze.

Keywords: GluR1, hippocampus, LTP, working memory

Utilization of genetic tools for studying neural development in avian embryos

Rendelman Z., Hazanov S., Guy U., Marom K., Feinstein Y. and $\mathrm{Klar} A$.

Dept. of Anatomy and Cell Biology, Hebrew University Hadassah Medical School, Jerusalem 91120

The employment of genetic tools for studying biology in general, and developmental biology in particular, has advanced our knowledge tremendously over the last decades. The ability to inactivate a specific gene, or to mis-express it, thereby generating a loss or gain-of-function mutant, is the ultimate way to verify its biological role. So far these tools were applied successfully in invertebrates and mice.

The avian embryo is the preferred organism for studying embryonic development of vertebrate. The embryo is flat, develops in ovo, and accessible for surgical manipulations. Hence, the inability to introduce genes in vivo has hampered the progress of research with this organism. In the last few years, a new method, electroporation, was introduced to deliver genes in vivo, and been used effectively in avian embryos. However, the site of expression is dictated by the topography of the embryo and the electrode. In order to achieve site-specific expression we have used electroporation of tissue specific enhancers. The activity of the specific enhancers was further amplified by the use of DNA site-specific recombinase. In this way, we are able to achieve high level ectopic expression of an active form or a dominant negative form of a protein, at a specific site. For generating a null phenotype, we are currently applying the RNAi method in chick embryos in vivo.

The genetic tools will be presented, and their potential use for studying axon guidance and cell migration during early development will be demonstrated.

Keywords: axon guidance, transgenic chick

Setting apart the affected: the use of behavioral criteria in animal models of Acute Stress Response and Post

Traumatic Stress Disorder

Richter-Levin G. ${ }^{1}$, Avital A. ${ }^{1}$, Matar M. ${ }^{2}$, Zohar J. ${ }^{3}$, Kaplan $Z^{2}$ and Cohen $\mathrm{H}$

${ }^{1}$ Dept. of Psychology, University of Haifa, ${ }^{2}$ Ministry of Health Mental Health Center, Anxiety and Stress Research Unit,

Faculty of Health Sciences, Ben-Gurion University of the Negev, Beer-Sheva, ${ }^{3}$ The Chaim Sheba Medical Center, Sackler Medical School, Tel-Aviv University

Post-traumatic stress disorder affects only $20-30 \%$ of the exposed population. Clinical studies of acute and chronic responses to stress generally employ stringent criteria for inclusion in the study population, and yet in animal studies the data are usually expressed as a function of the entire exposed versus the entire unexposed populations, regardless of individual variation in response. Prior data support an approach to animal models analogous to inclusion criteria in clinical studies.
This series of studies sought to assess prevalence rates of maladaptive versus adaptive responses determined according to two successive behavioral tests (elevated plus maze/open field and acoustic startle response tests), amongst rats exposed to a variety stress paradigms, in the acute and chronic phases, for single and repeated exposures, early in life and/or in adulthood. The results shed light on the pattern of prevalence rates of maladaptive responses to stress over time, with rates dropping from $90 \%$ acute responders to a plateau at about $25 \%$ enduring/chronic response. This plateau is attained at seven days and remains unchanged at 30 days in two distinct study models. Different types of trauma are associated with different degrees of response, possibly reflecting a "dose-response" relationship to severity of trauma. Re-traumatization is associated with increasing prevalence of maladaptive responses, all the more so for early-life trauma repeated in adulthood.

Setting the affected individuals apart from the unaffected and focusing on them clarifies the overall picture and more closely approximates clinical studies

Keywords: Post-traumatic Stress Disorder, animal model, anxiety, stress, maladapted, well adapted

\section{Studying architecture of $\mathbf{G}$-protein coupled $\mathrm{K}^{+}$channels} using fluorescence based approaches

Riven I., Kalmanzon E., Segev L. and Reuveny E Dept. of Biological Chemistry, Weizmann Institute of Science, Rehovot 76100

G protein coupled potassium channels (GIRK/Kir3.x) translate inhibitory chemical neurotransmission into changes in cellular excitability. These channels are activated by binding of the $\mathrm{G} \beta \gamma$ subunits to $G$ proteins, and are regulated by other intracellular components as well. To understand the mechanism of channel activation by $G$ proteins it is necessary to define the structural rearrangements in the channel that result from interaction with $\mathrm{G} \beta \gamma$ subunits. In this study we used a combination of fluorescence spectroscopy and through-the-objective total internal reflection microscopy to monitor the conformational rearrangements associated with the activation of GIRK channels. Conformational changes were assessed from changes in the efficiency of fluorescence resonance energy transfer (FRET) between CFP and YFP attached at various positions in the cytosolic domains. We detect activation-induced changes in FRET consistent with a rotation and expansion of the termini along the central axis of the channel. We propose that this rotation and expansion of the termini drives the channel to open by bending and possibly rotating the second transmembrane segment.

Wasp venom injected into prey's brain affects the activity of bioaminergic neurons to regulate the expression of specific motor patterns Rosenberg L.A. and Libersat F. Zlotowski Center for Neuroscience and Dept. of Life Sciences, Ben-Gurion University of the Negev, Beer-Sheva, Israel

The parasitoid wasp Ampulex compressa hunts cockroaches to serve as a living food supply for its larvae. The wasp injects its venom directly into the brain to induce a lethargic state lasting about five weeks. While in this state, the cockroach does not escape. Our hypothesis is that the injected venom affects neurons located in the brain, which send descending tonic input to bioaminergic neurons; these, in turn, control the thoracic premotor circuitry. Octopamine is a bioamine, which is secreted by DUM (dorsal unpaired median) neurons in the prey's thoracic portion of the nervous system. It is known to regulate the expression of specific motor patterns by modulating the excitability of specific neurons. In this work, we show that the activity of DUM neurons is altered in stung animals. Identified DUM neurons in the metathoracic ganglia of stung animals have a lower spontaneous firing rate and their responsiveness to specific sensory stimuli decreases. For example, DUM234 neuron does not produce a burst of action potentials in response to a wind stimulus as it does in control animals.

Given these results, we propose that the venom injected into the brain affects descending tonic input to the thoracic DUM neurons. The decrease in activity of the DUM neurons could be responsible for the decrease in the responsiveness of thoracic motor circuits and consequently the expression of specific motor patterns such as the escape behavior

Keywords: ampulex compressa, cockroach, octopamine, dorsal unpaired median neurons 
Genetic variations in apoptosis and inflammation-related genes and the risk of Alzheimer's disease

Rosenmann H. ${ }^{\text {, }}$, Meiner Z. ${ }^{1}$, Kahana E. ${ }^{2}$, Aladjem Z. ${ }^{2}$ Friedman G. ${ }^{3}$, Ben-Yehuda A. ${ }^{3}$, Grenader T. ${ }^{3}$, Wertman E. and Abramsky $\mathrm{O}^{1}$

The Agnes Ginges Center for Human Neurogenetics, Dept. of Neurology and ${ }^{\Phi}$ Geriatric Unit, Hadassah University Hospital, Jerusalem: ${ }^{2}$ Dept. of Neurology, Barzilai Medical Center, Ashkelon; ${ }^{4}$ Dept. of Neurogeriatrics, Herzog Memorial Hospital, Jerusalem.

The etiology of sporadic Alzheimer's disease (AD) is complex, and both genetic and environmental factors are involved. The APOE-e4 variant the only widely confirmed major risk factor for $\mathrm{AD}$ accounts for only part of the genetic component of $\mathrm{AD}$, and other genetic variants are yet to be detected.

We performed association studies in 109 sporadic AD cases and 111 aged healthy controls. We investigated natural genetic variations (polymorphisms) in 5 genes selected by pathobiological criteria: p53 and TNFRSF6 (Fas), both apoptosis-related genes which are upregulated in AD brains; complement component (Clr) and interleukin-1-beta (ILl-B), both inflammation-associated genes upregulated in AD brains; and heparane sulfate proteoglycan (HSPG2), which is associated with amyloid plaques and neurofibrillary tangles. We tested whether these genes act as susceptibility genes affecting the risk for $A D$ or as modifier genes influencing its clinical features. While our study population demonstrated a strong association between $\mathrm{AD}$ and the APOE-e4 risk factor $(p=0.000002)$, no such association was found with the following polymorphisms: (codon 72) p53 [p=0.921], (-670) Fas $[\mathrm{p}=0.475]$, (codon 135)Clr $[\mathrm{p}=0.353]$, (3953)IL 1-B $[p=0.628]$ and (domain I)HSPG2 $[\mathrm{p}=0.486]$. No association was detected also for age at disease onset and disease progression, and no interactive effect was found with APOE-e4. This is the first report of association studies of $\mathrm{p} 53$, $\mathrm{C} 1 \mathrm{r}$ and HSPG2 with AD or any neurodegenerative disorder at all. Our results of Fas and IL1-B are in agreement with initial reports, published during our work. Further intensive association studies are needed for identifying genetic risk factors that will allow constructing a profile of individuals prone to $\mathrm{AD}$, and may assist in developing novel therapies. Keywords: Alzheimer's disease, polymorphism, apoptosis, inflammation, proteoglycan.

\section{Microglia and macrophage activation in the injured nervous system}

Rotshenker S., Reichert F., Slobodov U. and Makranz C. Dept. of Anatomy and Cell Biology, the Eric Roland Center for Neurodegenerative Diseases, Hebrew University Faculty of

Medicine, Jerusalem

Microglia and macrophages play critical roles in the response of the CNS and PNS to injury and disease. One major role is the removal of degenerating myelin by phagocytosis. Myelin degeneration occurs after injury to axons and in autoimmune demyelinating diseases such as multiple sclerosis and EAE. Degenerated myelin inhibits axonal regeneration and further activates the complement system to form membrane attack complexes that disintegrate intact myelin. The rapid removal of degenerating myelin is vital, therefore, for repair and minimizing additional damage to myelin.

Myelin phagocytosis can be mediated by complement-receptor-3 (CR3/MAC-1), scavenger receptor (SRAI/II) and Fc-receptor (FcR). We presently document that CR3/MAC-1 is expected to play the major role in mediating myelin phagocytosis after trauma $(70 \%$ to $80 \%)$. However, its in-vivo expression does not indicate necessarily the occurrence of myelin phagocytosis. CR3/MAC-1 expressing microglia are fully activated and phagocytose mvelin efficiently in EAE but not after injury to CNS axons. These observations led us to suggest that CR3/MAC-1 mediated myelin phagocytosis is subjected to modulation such that it may range between efficient and inefficient states.

We tested the functional plasticity of CR3/MAC-1 mediated myelin phagocytosis in macrophages and microglia by anti-CR3/MAC-1 mAbs, documenting inhibition and augmentation of myelin phagocytosis by anti-CR3/MAC-1 mAbs. We suggest that mAbs binding to distinct extracellular epitopes in $\alpha / \beta$ subunits of CR3/MAC-1 can modulate (inhibit or augment) CR3/MAC-1 mediated myelin phagocytosis by inducing and/or stabilizing distinct conformational changes that correspond with inactive or active functional states CR3/MAC-1. We further suggest that anti-CR3/MAC-1 mAbs may reveal a mechanism by which native extracellular molecules bind to and modulate CR3/MAC-1 mediated myelin phagocytosis in microglia and macrophages. Keywords: trauma, regeneration, microglia, macrophages, myelin
Brain reward system and depression: A tool for testing hedonia in an animal model

Roth Deri I., Gispan-Herman I. and Yadid G. Faculty of Life Sciences, Bar-Ilan University

Anhedonia is one of the main parameters that distinguish a depressed patient from a normal one. However, the brain mechanisms that underlie anhedonia remain elusive. Data suggest that there are overlaps between depression/anhedonia and neural reward systems. Therefore, the brain reward system may be a potentially important therapeutic target to relieve anhedonic symptoms.

The Flinders sensitive line of rats (FSL) is a valid and reliable model of depression with strong face-, construct- and predictive- validity. Abnormalities in the reward system of FSL rats were tested behaviorally and neurochemically using the cocaine self-administration paradigm in parallel with microdialysis measurement of dopamine levels in the nucleus accumbens. Our results show that cocaine consumption was significantly higher in control than in FSL rats. Treatment with the antidepressant desipramine increased the cocaine self-administration in FSL rats. Basal dopamine secretion ratio (using GBR 12909) and extracellular dopamine in response to cocaine were also abnormal in the nucleus accumbens of FSL rats. Both of these measures were normalized following treatment with desipramine. Taken together, we suggest that the brain reward system may be a tool for studying the anhedonia expressed in depressive behavior.

Keywords: brain reward system, cocaine, hedonia, animal model

Plasma Homocysteine in Schizophrenia

Ruderman V., Stahl Z., Ben-Ami S., Belmaker RH. and Levine

Stanley Foundation Research Center, Ministry of Health Mental Health Center, Faculty of Health Sciences, Ben Gurion University of the Negev. Beer Sheva

Elevated plasma homocysteine has been found to be a risk factor for Alzheimer's disease as well as in cerebral vascular disease, suggesting that some risk factors can accelerate or increase the severity of several CNS disease processes. We screened chronic schizophrenic patients in our catchment area for plasma homocysteine levels

A one-way ANCOVA with age and sex as covariants was performed on plasma total homocysteine levels of 190 schizophrenic patients vs 762 controls (evaluated in a screening program for employee health). The effect of schizophrenia was marked $(\mathrm{F}=130.5 \mathrm{df}=1.902, \mathrm{p}<0.0001)$ and mean homocysteine level was $16.7 \pm 11.8$ (SD) in schizophrenic patients vs $10.6 \pm 3.6$ (SD) in healthy controls (covariance adjusted means 16.5 vs 10.7 in controls). The increase was almost entirely in young male schizophrenic patients.

Homocysteine has been shown to be neurotoxic and it has been shown that stress can open the blood brain barrier to some neurotoxic substances. It is possible that the stress of acute psychosis allows high homocysteine levels to enter the brain and cause neurodegeneration, clinical deterioration and chronicity. This hypothesis does not make any assumption as to the origin of high homocysteine in young male schizophrenics. It could be caused by smoking, lack of exercise or by poor diet. Yet via a biochemical mechanism it could negatively affect the course of illness.

Keywords: homocysteine, neurodegeneration, schizophrenia

The degree of monoamine oxidase inhibition by TV3326 in relation to MPTP induced neurotoxicity and $L$-dopa

induced dopamine behavioral syndrome

Sagi ${ }^{1}{ }^{1}$, Weinstock $\mathrm{M}^{2}$ and Youdim $\mathrm{MBH}^{1}$

${ }^{T}$ Eve Topf and NPF Centers of Excellence for

Neurodegenerative Diseases Research, and Dept. of

Pharmacology, Technion- Faculty of Medicine, Haifa $;{ }^{2}$ Dept.

of Pharmacology, Hebrew University, Jerusalem

TV3326 [(N-propargyl-(3R)-aminoindan-5-yl)-ethyl methyl carbamate])] is a novel bifunctional cholinesterase $(\mathrm{ChE})$ as well as brain-selective monoamine oxidase (MAO) A and $\mathrm{B}$ inhibitor, which has been developed for treatment of dementia with extra pyramidal disorder such a s Lewy body disease. In vitro TV3326 has little or no MAO inhibitory activity. However, on chronic treatment $(26 \mathrm{mg} / \mathrm{kg} /$ day for 14days) in mice it inhibits both brain MAO iso-enzymes by $\sim 70 \%$, resulting in a complete protection from MPTP (1-methyl-4-phenyl-1,2,3,6-tetrahydropyridine)-induced neurotoxicity of nigrostriatal dopamine neurons. The neurotoxicty of MPTP is dependent on its conversion to the neurotoxin MPP+ (1-methyl-4-phenylpyridinium ion) by MAO $\mathrm{B}$. It is not known what degree of MAO B inhibition is required for neuroprotection against MPTP. In the present 
study we show that protective effect of TV-3326 is not dependent on complete inhibition of MAO B. A correlation exists between degrees of MAO $B$ inhibition and neuroprotection as measured by striatal dopamine concentration. In addition the effect of TV $-3326(52 \mathrm{mg} / \mathrm{kg})$ on the 1-dopa induced dopamine behavioral stereotypy was evaluated in rats and compared to that initiated by the non-selective MAO inhibitor tranylcypromine (TCP) $(10 \mathrm{mg} / \mathrm{kg})$. Both TV -3326 and TCP as expected, caused highly significant increases in striatal dopamine. However, the behavioral syndrome that is normally seen with TCP and other MAO inhibitors plus 1-dopa was absent with TV3326. These results in part may be explained by inhibitory effect of increased acetylcholine levels on MI receptors resulting from $\mathrm{ChE}$ inhibition by TV-3326.

Keywords: TV-3326, MAO, ChE, MPTP

SKF-100303A, an inhibitor of the GABA transporter GAT1, regulates chloride concentration in SCN neurons Sagiv N. ${ }^{1}$ Belenky M. ${ }^{2}$ and Yarom Y.

Depts. of ${ }^{1}$ Neurobiology and ${ }^{2}$ Cell and Animal Biology,

Institute of Life Sciences, Hebrew University, Jerusalem

We have shown that GABA has a dual effect on SCN neurons, excitatory during the day and inhibitory at night (Wagner et al. Nature 387: 598-603 [1997]). This duality, which has been attributed to changes in intracellular $\mathrm{Cl}^{-}$concentration, is due to changes in chloride regulation (Wagner et al. J. Physiol. 537: 853-869 [2001]). We investigated the role of the GABA transporter, GAT1, in regulating chloride concentration in SCN neurons. This transporter, which in the SCN is located on axon terminals and glia, as well as on some dendrites, translocates GABA along with chloride and sodium. We studied the effect of the GAT1 inhibitor SKF-100303A on the decay time of GABA currents, induced by either inhibitory synaptic activity or by local application of GABA. The blocker caused prolongation of the decay time constant by an average of 35\%, and had a larger effect on inward (39\%) than on outward $(31 \%)$ currents. We next investigated the effect of the blocker on the recovery of intracellular $\mathrm{Cl}^{-}$concentration. Loading or depletion of intracellular $\mathrm{Cl}^{-}$was induced by a prolonged GABA application, which generated either influx or efflux of chloride, depending on the membrane holding potential. Recovery was deduced from the change in chloride reversal potential calculated from the response to a test GABA pulse presented at $30 \mathrm{sec}$ delay after the first GABA pulse. While recovery from chloride loading was nearly unaffected by the blocker, recovery from chloride depletion was reduced, particularly during subjective day.

Keywords: SCN, circadian rhythm, GABA

\section{Single trial and artifact extraction from human evoked} potentials using sparsity

Sandhaus H., Meir R., Zibulevsky M. and Pratt H.

Evoked Potentials Laboratory, Technion-Israel Institute of Technology, Haifa; Dept. of Electrical Engineering,

Technion-Israel Institute of Technology, Haifa

Event-related potentials (ERPs) have a low amplitude relative to background EEG activity. Therefore, ERPS are usually averaged, disregarding variations in the time course of activity among the single trials comprising the average. This study addressed this need for ERP single trial derivation by solving the blind source separation (BSS) problem.

In the BSS problem unknown sources that are linearly mixed with an unknown mixing matrix need to be extracted based only on the mixtures. Most approaches that solve this problem rely on independence between the sources (ICA) or some distribution quality. Sparsity assumes that only a small number of signal values differ significantly from zero. Biological signals can be sparsely represented in a Fourier subset such that each source has a sparse representation by its decomposition Fourier coefficients. This criterion is used without any additional assumptions in order to separate sources of EEG. The data used in order to demonstrate the algorithm's performance included responses to two types of stimuli randomly presented in sequence using the "odd-ball" paradigm. The separated sources of the multichannel data show that artifacts such as eye movement, eye blink, ECG and in some subjects muscle activity, are represented by separate sources Moreover, frontal activity ( $\mathrm{Nl}$ and $\mathrm{P} 2$ waves) and parietal activity ( $P 3$ wave) were separately represented. ERP component templates were used in addition to the sparsity criterion in order to improve the ERP signal/noise ratio. A raster display of single trials allowed following the dynamics of each component during the recording session.

Keywords: Blind Source Separation (BSS), ERP single trial, sparsity
Paxb and En1 regulate distinct steps in the development of renshaw cells

Sapir T. ${ }^{1}$, Geiman E.G., Velasquez T. ${ }^{1}$ Alvarez F.J. ${ }^{2}$ and Goulding $\mathrm{M}$.

${ }^{I}$ Molecular Neurobiology Laboratory, The Salk institute, La

Jolla, CA; ${ }^{2}$ Dept. of Anatomy, Wright State University.

Dayton, $\mathrm{OH}$

During development functional classes of neurons are selectively generated at defined locations according to the position their progenitors along the neuroaxis. In the ventral spinal cord, graded Shh signaling subdivides the neuroepithelium into five progenitor domains that give rise to different subtypes of neurons. It is not known whether these embryonic subtypes define physiologically identified neuronal classes in the adult, nor is it known how genes that are transiently active in these neurons contribute to the formation of locomotor circuits.

Previously, we demonstrated that V1 IN's are locally projecting inhibitory interneurons that express GAD65. To study the V1 population at late stages of development we created En $1{ }^{\text {Cre }}$ knockin and Rosa- ${ }^{26 \mathrm{Gap} 4-\mathrm{EGFP}}$ reporter mice. We have shown that the V1 interneurons are last order interneurons and directly synapse onto motor neurons. We also show that a subpopulation of $\mathrm{V} 1$ interneurons $(10 \%)$ differentiate into adult Renshaw cells and that adult Renshaw cells are exclusively derived from Vl interneurons. Gephyrin and calbindin immunoreactivity identification criteria and anterograde transynaptic transport from the motor axon of an EGFP-TTC were used to define adult Renshaw cells. We have found that Renshaw cells are not generated in Pax6 mutant mice. In contrast, Enl mutant mice have normal numbers of Renshaw cells; however Renshaw cell-motor neuron connectivity is altered. These results demonstrate that Pax6 and En1 control two distinct steps in the development of the spinal cord circuit that mediates recurrent inhibition of motor neurons.

Supported by grants from the National Institutes of Health and the Christopher Reeve Paralysis Foundation.

Keywords: En1, Pax6, Renshaw cells

Correcting space-clamp distorted voltage-clamp recordings from dendrites

Schaefer A.T. ${ }^{1}$, Helmstaedter M. ${ }^{1}$, Sakmann B. ${ }^{1}$. and Korngreen A 1 , 2

Abteilung Zellphysiologie, Max-Planck-Institut fuer

Medizinische Forschung Jahnstrasse 29, D-69120 Heidelberg Germany; 1,2 Faculty of Life Sciences, Bar-Ilan University, Ramat-Gan 52900, Israel

Understanding the functions of a single neuron, such as, propagation and generation of synaptic or action potentials, a detailed description of the kinetics and distribution of the underlying ionic conductances is essential. In voltage-clamp experiments, incomplete space clamp distorts the recorded currents, rendering accurate analysis impossible. We developed a simple numerical algorithm that corrected such distortions The method performs a stepwise approximation of the conductance density at the site of a local voltage clamp. This is achieved by estimating membrane conductances in a simulation that yields simulated clamp currents, which are then fitted to the distorted recordings from the non-space-clamped structure, relying on accurately reconstructed cell morphology and experimentally determined passive properties. The algorithm was tested using channels with simplified kinetics and on published realistic potassium-channel models. For all tested conditions, the method enabled accurate retrieval of the local densities and kinetics of somatic and dendritic channels at the site of the voltage clamp, rather than averaging these parameters across the structure. Furthermore, correct calculation of conductance gradients required only a few recordings. Neither the addition of noise nor variation of passive parameters significantly reduced the performance of the correction algorithm. The correction method was applied to two-electrode voltage-clamp recordings of $\mathrm{K}^{+}$currents from the apical dendrite of layer 5 neocortical pyramidal neurons. The generality and robustness of the algorithm make it a valuable tool for voltage-clamp analysis of voltage-gated currents in structures of any morphology that is amenable to the voltage-clamp technique.

Keywords: voltage-Clamp, potassium channels, cable-theory

The representation of objects in the human lateral occipital complex

Schiff M. and Zohary E.

Dept. of Neurobiology, Hebrew University, Jerusalem 91904

Regions in the lateral and ventral occipital cortex, known as LOC, play an important role in human object recognition. LOC is characterized by a stronger response to objects compared to 
scrambled objects or textures, regardless of the visual cues used to define object shape (luminance, texture or motion differences). Can this cue invariance be observed at the level of single neurons, or are different neuronal populations within LOC sensitive to different object cues. To test this we utilized the fact that LOC shows fMRI-adaptation, a decreased activation in response to repeated stimulus presentations. Our subjects watched two types of three-dimensional objects: (1) stationary, in which object shape was defined by its contours, shading, etc, and (2) structure from motion (SFM) stimuli, in which the perception of an object is induced solely from motion cues. Every SFM object was made of 18 different frames, depicting the rotating object as it is seen from sequential viewpoints. The object was unidentifiable from any single frame. Both types of stimuli activated LOC, and in both cases fMRI-adaptation was seen following stimulus repetition. Since there is little physical similarity between successive SFM images, adaptation during the SFM repetition indicates that LOC is involved with holistic object identity. However, when stationary pictures and SFMs of the same object were alternated within a block no adaptation was apparent. This may imply that while on the macroscopic level LOC is indeed processing object information in a cue invariant manner, separate neuronal groups within LOC process different object cues.

Keywords: LOC, structure from motion, adaptation

The role of orbitofrontal cortex and basolateral amygdala in latent inhibition - A possible model of negative symptoms in schizophrenia

Schiller D. and Weiner I.

Dept. of Psychology, Tel Aviv University, Tel Aviv 69978

Latent inhibition (LI) refers to the proactive interference of inconsequential stimulus pre-exposure with its ability to signal significant events, and disrupted LI is considered to model positive symptoms of schizophrenia. Lesions of prefrontal cortex (PFC) and basolateral amygdala (BLA), that were reported to produce behavioral effects potentially relevant to schizophrenic symptomatology in several animal models, have been reported to spare LI. However, certain drug and lesion manipulations produce abnormally persistent rather than disrupted LI and we have suggested that such LI perseveration may model the impaired set shifting associated with negative symptoms of schizophrenia. In the present study, we tested whether excitotoxic lesion of BLA and orbitofrontal cortex (OFC), which are reciprocally connected, will induce LI perseveration, which would become evident under conditions that do not allow for LI in controls. LI was measured in a thirst motivated conditioned emotional response procedure by comparing suppression of drinking in response to a tone in rats which received 0 (nonpre-exposed) or 40 tone presentations (pre-exposed) followed by either 2 or 5 tone-shock pairings. Control rats showed LI with 40 pre-exposures and 2 conditioning trials, but raising the number of conditioning trials to five disrupted LI. In contrast, both OFC- and BLA-lesioned rats persisted in exhibiting LI under the latter conditions. PFC and amygdalar pathology has been extensively reported to be an essential feature of schizophrenia, thus, our results that OFC and BLA damage lead to persistent rather then disrupted LI, indicate that persistent LI provides an animal model of such deficits in schizophrenia.

Keywords: basolateral amygdala, orbitofrontal cortex, latent

inhibition, schizophrenia

Recurrent electrical stimulation suppresses inter-ictal like discharges an electrographic seizures in acute models of neocortical epilepsy in-vitro

Schiller Y.

Dept. of Neurology, Rambam Medical Center, Haifa

Background: In most patients with epilepsy, seizures can be controlled by antiepileptic drugs. However, approximately a fifth of patients with epilepsy suffer from an intractable disease and continue to experience seizures despite appropriate medical treatment. Hence, new antiepileptic treatment strategies are required for intractable epilepsy. One potential new antiepileptic treatment modality is electrical stimulation Vagal nerve stimulation is already approved for treatment of intractable epilepsy. In addition it is possible that seizures can be eliminated by cortical electrical stimulation of the epileptogenic zone.

Objectives: Examine the ability of chronic electrical stimulation to eliminate inter-ictal epileptiform discharges and electrographic seizures in acute models of epilepsy in-vitro. Methods: The study was performed in neocortical brain slices treated with either the GABA-A receptor blocker Bicucculine (BCC, $10 \mu \mathrm{M})$ or zero extra-cellular magnesium. Recordings were performed using whole-cell recordings from single neurons or extra-cellular recordings from a population of neurons. Electrical stimulation was performed using a computer controlled extra-cellular stimulator.

Results: Both acute models of neocortical epilepsy produced inter-ictal like discharges and electrographic seizures. Recurrent electrical stimulation at frequencies of $0.2-2 \mathrm{~Hz}$ eliminated altogether inter-ictal like discharges and electrographic seizures in both BCC treated slices and slices exposed to zero-magnesium. To investigate possible mechanisms underlying the antiepileptic effect of recurrent electrical stimulation I examined its effect on excitatory synaptic transmission. I found that recurrent electrical stimulation resulted in a $20-55 \%$ reduction of the EPSP amplitude in a frequency dependent manner.

Conclusions: Recurrent electrical stimulation has an antiepileptic effect in acute models of neocortical epilepsy in-vitro. This effect is probably mediated, at least in part, by stimulus-evoked depression of excitatory synaptic transmission. Further studies are required to examine the antiepileptic effect of electrical stimulation in-vivo.

Keywords: epilepsy, seizures, electrical stimulation

Field-specific changes in hippocampal opioid mRNA, peptides, and receptors due to prenatal morphine exposure in adult male rats

Schindler C.J. ${ }^{1 *}$ Slamberová $\mathbb{R}^{2,3}$, Rimanóczy Á. ${ }^{2,4}$, Hnactzuk O. ${ }^{1}$, Riley M.A. ${ }^{p}$ and Vathy I. Depts. of ${ }^{1}$ Neuroscience and ${ }^{2}$ Psychiatry and Behavioral Sciences, Albert Einstein College of Medicine, Bronx, NY USA; ${ }^{3}$ Dept. of Normal, Pathological and Clinical Physiology, 3 rd Faculty of Medicine, Charles University, Prague, Czech Republic; ${ }^{7}$ Dept. of Psychiatry, Albert Szent-Györgyi Center for Medical and Pharmaceutical Sciences, Faculty of Medicine, University of Szeged, Szeged, Hungary; *Current affiliation: Faculty of Life Sciences, Bar Ilan University

Alterations in the opioid system in the hippocampus as well as some possible functional consequences were investigated by several methods in adult male rats who were prenatally exposed to either saline- or morphine $(10 \mathrm{mg} / \mathrm{kg}$ twice daily on gestational days 11-18). In situ hybridization and Northern blots were used to measure proenkephalin and prodynorphin mRNA, and radioimmunoassays were used to quantify proenkephalin- and prodynorphin-derived peptide levels in the dentate gyrus (DG), CA3, and CA1 subfields of the hippocampus. Prenatal morphine exposure in male rats decreases proenkephalin and increases prodynorphin mRNA selectively in the granule cell layer of the DG. Similarly, met-enkephalin peptide levels are decreased and dynorphin $B$ peptide levels are increased in the DG but not CA 3 or CA1 of prenatally morphine-exposed males. In addition, there are decreases in some dynorphin-derived peptides in the CA3 subfield. Receptor autoradiography revealed increases in the density of $\mu$ but not $\delta$ receptor labeling in discrete strata of specific hippocampal subfields in prenatally morphine-exposed males. Prenatal morphine exposure, however, does not alter the latency to onset or number of episodes of wet dog shakes or clonic seizures induced by infusion of $10 \mathrm{nmol}$ [D-Ala ${ }^{2}$ $\mathrm{MePhe}^{4}, \mathrm{Gly}^{-0 l^{5}}$ ] enkephalin (DAMGO) into the ventral hippocampus. Interestingly, a naloxone $(5 \mathrm{mg} / \mathrm{kg}$ ) injection 30 min before bicuculline reverses the increased latency to onset of clonic and tonic-clonic seizures in prenatally morphine-exposed males. Thus, the present study suggests that prenatal morphine exposure alters the hippocampal opioid system, suggesting possible alterations in the excitability of the hippocampus.

Keywords: drug exposure, epilepsy, enkephalins, dynorphins; peptides, hippocampal layers

Parental deprivation induces changes of distinct shape parameters of deneritic spines in the anterior cingulate cortex of octodon degus

Schnabel R. ${ }^{1}$, Herzog A. ${ }^{2}$, Michaelis B. ${ }^{2}$ and Braun K. ${ }^{1}$ *I Otto-von-Guericke University. Inst. Biol., Brenneckestr 6. 39118 Magdeburg ${ }^{2}$ Inst. Electr. Signal Process. Commun. 39016 Magdeburg, Germany;

Email: katharina.braun@nat.uni-magdeburg.de

The synaptic development of higher associative cortical areas such as the anterior cingulate cortex (ACd), displays a high sensitivity towards environmental influences, such as severe uncontrollable psychosocial stress. An indicator for such experience-induced changes of synaptic efficacy may be alterations of synaptic shape. In the present study we searched for differences in the fine morphological parameters of dendritic spines on the basal dendrites of Lucifer Yellow-injected layer III pyramidal neurons in the ACd of 
parentally deprived $(3 \times 1$ hour per day between postnatal days 1 and 21), 45 days old degus in comparison with age-matched socially undisturbed control pups. The 3D-measurements of spines were performed in image stacks produced by a confocal laser-scanning microscope applying a newly developed image analysis software (Herzog et al., SPIE 2984:146-158, 1997). Although the spine volumes remained unchanged, a significant reduction of spine length, accompanied by a significant increase of mean spine diameter was observed in the parentally deprived animals. These overall effects were due to a reduction of spine length on dendritic branch 2 and 4, and a significant increase of spine diameter on branch 3 . This is the first evidence that the disturbance of the parent-child contact during an early postnatal phase results in lasting changes of distinct shape parameters of presumed excitatory spine synapses, which most likely reflect a experience-related fine-tuning of synaptic strength within limbic cortical circuits.

Supported by grants from the Volkswagenstiftung and SFB 426.

Keywords: limbic system, development, synaptic plasticity, dendritic spines

\section{Immune related mechanisms participating in resistance} and susceptibility to glutamate toxicity

Schori H. ${ }^{\text {. }}$ and Schwartz M.

Dept. of Neurobiology, Weizmann Institute of Science, Rehovot 76100

Glutamate is an essential neurotransmitter in the central nervous system (CNS). However, at abnormally high concentrations it becomes cytotoxic. Recent studies in our laboratory showed that glutamate evokes $\mathrm{T}$ cell-mediated protective mechanisms,. The aim of the present study was to examine the nature of the glutamate receptors and signaling pathways that participate in immune protection against glutamate toxicity. We show, using the mouse visual system, that glutamate-induced toxicity is strain dependent, not only with respect to the amount of neuronal loss it causes, but also in the pathways it activates. In strains that are genetically endowed with the ability to manifest a $\mathrm{T}$ cell-dependent neuroprotective response to glutamate insult, neuronal losses due to glutamate toxicity were relatively small, and treatment with NMDA-receptor antagonist worsened the outcome of exposure to glutamate. In contrast, in mice devoid of $T$ cell-dependent endogenous protection, NMDA receptor antagonist reduced the glutamate-induced neuronal loss. In all strains, blockage of the AMPA/KA receptor was beneficial. The results suggest that glutamate-induced toxicity involves multiple glutamate receptors and that the types and relative contributions of receptors vary among strains. We suggest that a multi-factorial protection, based on a mechanism independent of the specific pathway through which glutamate exerts its toxicity, is likely to be a safer, more comprehensive, and hence more effective strategy for neuroprotection.

Keywords: glutamate, NMDA, immune system, neuroprotection, CNS

\section{An 55 residue of KCNQ1 controls channel gating and pore} properties and is essential for the specific interaction with KCNE3

Schottelndreier H., Peretz A. and Attali B.

Sackler School of Medicine. Dept. of Physiology \&

Pharmacology, Tel Aviv University, Tel-Aviv 69978

The KCNQ1 pore-forming subunit is a member of the newly characterized $\mathrm{K}^{+}$channel family (KCNQ1-5), whose mutations have been identified as causes of inherited cardiovascular and neurological disorders such as LQT syndrome and neonatal epilepsy. KCNQ1 can interact with KCNE proteins, an emerging family of auxiliary $\beta$ subunits, to produce functionally distinct $\mathrm{K}^{+}$currents. Here we show that the naturally occurring LQT1 mutation L273F, located in the S5 transmembrane segment of the KCNQ1 pore-forming subunit leads to dramatic changes in channel gating and permeation as well as in the interaction properties with KCNE3. The L273F mutation produces a marked rightward shift of activation gating $(+28 \mathrm{mV})$ with a shallower slope of the voltage dependence of activation in comparison to WT KCNQ1. In addition, L273F generates a pronounced macroscopic inactivation that exhibits distinct properties to those displayed by WT KCNQ1, including slower recovery kinetics. In contrast to WT KCNQ1, external protons poorly inhibit the maximum conductance and the macroscopic inactivation of $\mathrm{L}_{273 \mathrm{~F}}$. Expression of WTKCNQ1 with WTKCNE3 leads to $\mathrm{K}^{+}$ currents that activate quasi instantaneously at all voltages, suggesting that heteromeric KCNQ1/KCNE3 channels are constitutively open and exhibit neither voltage- nor time-dependence. Co-expression of L273FKCNQ1 with WT KCNE3 dramatically changes channel gating and exhibits a biophysical profile resembling that produced by WTKCNQ1/WTKCNE1 ( $\left.\mathrm{I}_{\mathrm{KS}}\right)$; it leads to slowly activating outwardly-rectifying $\mathrm{K}^{+}$currents with a strong block of inward currents. In all, our results indicate that the KCNQ1 S5 residue L273 critically controls the channel gating and pore properties, probably allosterically, and is essential for the specific interaction with $\mathrm{KCNE} 3$

Keywords: $\mathrm{K}^{+}$channels, inactivation, gating

\section{$B$ vitamins and brain aging}

Selhub J.

Tufts University, Boston MA.

The vitamins folic acid, B12 and B6 and B2 are the source of coenzymes which participate in one carbon metabolism. In this metabolism, a carbon unit from serine or glycine is transferred to tetrahydrofolate (THF) to form methylene-THF which is used for the synthesis of thymidylate (DNA), purines (DNA, RNA) and methionine. Methionine is converted to S-adenosylmethionine, a universal donor of methyl groups, including DNA, RNA, hormones, neurotransmitters, membrane lipids, proteins and others. Interest has been growing particularly in the area of aging and the possibility that certain diseases that afflict the aging population, loss of cognitive function, Alzheimer's disease, cardiovascular disease, cancer and others, may be in part explained by inadequate intake or inadequate status of these vitamins. Homocysteine, a product of methionine metabolism as well as a precursor of methionine synthesis, was shown recently to be a risk factor for cardiovascular disease, stroke and thrombosis when its concentration in plasma is slightly elevated. There are now data which show association between elevated plasma homocysteine levels and loss of neurocognitive function and Alzheimer's disease. In the Framingham Study we have shown that high plasma homocysteine is associated with increased incidence of dementia and Alzheimer's disease. There are many factors that contribute to a low status of these vitamins in the elderly and effort is now being placed to determine the beneficial effect of homocysteine lowering intervention on incidence of age related brain dysfunctions.

A role of the cholinergic system in depressive behavior Shabat M. ${ }^{1}$, Kinor N. ${ }^{1}$, Gispan-Herman I. ${ }^{1}$, Overstreet D.H. ${ }^{2}$ and Yadid $\mathrm{G}$.

${ }^{I}$ Faculty of Life Sciences, Bar-Ilan University Israel ; ${ }^{2}$ Dept. of Psychiatry, University of North Carolina, Chapel Hill, North Carolina, USA

Changes in the cholinergic system in the brain were one of the first neurochemical alterations discovered in depressive disorders. Since then, alterations in many more neurotransmitters were recognized. Using the Flinders sensitive Line (FSL) rats as a model of depression, we have found major changes in the neuronal monoaminergic systems. Recently, we discovered that specific and local molecular changes relate to the dynamics of depression onset in these rats. It was surprising to find that molecular changes in the limbic system were at terminal sites of the monoaminergic system and not in the cell body regions. This finding suggested that interneurons are involved in the pathology of depression and may serve as the common denominator in neurotransmitter alterations that are involved in the disease onset. The nucleus accumbens (Nacc) is likely to have a role in depression and contains cholinergic interneurons, which may mediate lasting behavioral changes by regulating monoamine release. To date, there has been no systematic investigation of the role of the cholinergic system in the Nacc in animal models of depression. Acute ( $45 \mathrm{~min})$ infusion of $5 \mu \mathrm{m}$ pirenzepine, a muscarinic-1 receptor antagonist, into the Nacc by a microdialysis probe returned the FSL's increased immobility in the swim test to the level of controls. Pirenzepine in FSL rats also normalized the basal levels of dopamine and serotonin in the extracellular space in the Nacc. These results may suggest that the cholinergic system is not just another neurotransmitter altered in depression, but is critical for controlling the interaction between several neurotransmitter systems. The convergence of these systems trigger the behavioral alterations found in depression. Keywords: cholinergic system, nucleus accumbens, muscarinic receptor, depression 
Stand-by microglial cells become activated glutamate scavengers upon T cell activation: Involvement of INF- $\gamma$ Shaked I., Butovsky O., Poart Z., Gersner R. and Schwartz M. Dept. of Neurobiology, Weizmann Institute of Science, Rehovot 76100

Inflammation following central nervous system (CNS) injury is still a subject of controversy, regarded by many as a detrimental process. Our group, however, have provided substantial evidence for immune involvement in neuronal protection. In this study we found that, the ability to resist the consequences of CNS axonal injury is characterized by the early activation of site-specific phagocytic activity and MHC class II expression. Our data suggest that post-traumatic CNS inflammation comprises a highly complex cascade of events, in which only a suitably timed and properly balanced innate immune dialog will lead to neuronal survival. Such a dialog is demonstrated in vitro by the ability of activated $T$ cells, via soluble factors, to activate microglial cells so as to increase their capacity to uptake extracellular glutamate. Interferon (IFN) $-\gamma$ was capable to simulate, at least in part, this activation effect. Using the RNA microarray methods we found in T cell-activated microglia cells alterations in expression of genes associated with an increase ability to resist threatening conditions, which often follows CNS lesion. These results suggest that autoimmune $T$ cells facilitate coping with injurious conditions by locally activating the stand by microglia cells. Keywords: CNS inflammation; CNS trauma; protective autoimmunity; glutamate toxicity; glutamate scavenging. microglia.

\section{Abnormal maternal behavior in GSK-3 $\beta$ knockout} heterozygote mice Shaldubina A ${ }^{1}$, Agam G. ${ }^{1}$, Hoeflich $\mathrm{K}^{2}{ }^{2}$, Woodgett J.R. ${ }^{2}$,

${ }^{Z}$ Zlotowski Center for Neuroscience, Faculty of Health

Sciences, Ben Gurion University of the Negev, Beer-Sheva;

${ }_{2}^{2}$ Ontario Cancer Institute/Princess Margaret Hospital, 610

University Avenue, Toronto, Ontario M5G 2M9, Canada

Glycogen synthase kinase-3 (GSK-3) is a multi-substrate serine/threonine protein kinase, highly abundant in brain. GSK-3 $\beta$ 's substrates include: transcription factors, regulatory enzymes and structural proteins and it is suggested to play a role in multiple cellular processes including: metabolism, proliferation, differentiation and development, particularly neurodevelopment. Its activity and protein levels have recently been reported to be over $40 \%$ lower in postmortem frontal cortex of schizophrenic patients. GSK-3 $\beta$ null mutant mice are unviable. Heterozygote mice, which have a $50 \%$ reduction in brain GSK-3 $\beta$, do not show any obvious physical or neurological abnormality. The GSK-3 $\beta$ heterozygotes are being studied in our lab for schizophrenic-like behavior. This experiment was conducted to test the maternal behavior of GSK-3 $\beta$ heterozygote female mice. Behavioral observation showed that heterozygote females have clear deficits in maternal behavior, leading to maternal infanticide. Neither pre-partum vanilla-smell on the mother's nose, nor pre-partum diazepam treatment prevented the infanticide. This finding could suggest a deficit in control of aggression or in bonding, and will be studied in specific behavioral paradigms.

Keywords: GSK, maternal behavior, knockout mice

Valproate inhibits Myo-inositol-1-phosphate (MIP) synthase

Shaltiel G. ${ }^{1}$, Agam G. ${ }^{1}$, Shamir A. ${ }^{1}$ and Greenberg $\mathrm{ML}^{2}$

Stanley Research Center, Zlotowski Center for Neuroscience, Psychiatry Research Unit, Ben Gurion University of the Negev, Beer-Sheva; ${ }^{2}$ Dept. of Biological Sicience, Wayne State University, Detroit, Michigan, USA

In mammalian brain, lithium inhibits inositol monophosphatase and both valproate and lithium reduce intracellular inositol The same reduction has been found in yeast where it was also observed that unlike lithium, that causes a parallel increase in intracellular inositol-1-phosphate, valproate causes a decrease in this metabolite. This suggested to us that myo-inositol-1-P (MIP) synthase is the site of valproate's action in inosito metabolism. MIP synthase is the rate-limiting step in inosito biosynthesis and in yeast is highly regulated in response to inositol. We therefore hypothesized that human brain MIP synthase is a factor in the psychopharmacology of mood stabilizers. The effect of therapeutically relevant valproate concentrations on MIP synthase activity in postmortem human brain homogenates was studied. Valproate inhibits human brain MIP synthase activity with a $\mathrm{Ki}$ of $0.21 \mu \mathrm{M}(0.35 \mu \mathrm{M}$ is the lowest plasma therapeutic level). The effect is not obtained with other anticonvulsant mood stabilizers, typical and atypical antipsychotics and tricyclic antidepressants. To find out whether reduction of inositol levels following lithium's inhibition of inositol monophosphatase or valproate's inhibition of MIP synthase is physiologically meaningful the chronic effect of lithium in food and the acute effect of i.p. valproate administration on brain MIP synthase expression was studied in mice. Lithium caused $33 \%$ upregulation of hippocampal MIP synthase expression and a two-fold upregulation of frontal cortex MIP synthase one hour after valproate treatment was observed. Inositol depletion as a first event in the therapeutic mechanism of action is thus common to lithium and valproate, albeit, through inhibition of different enzymes.

Keywords: lithium, valproate, Myo-Inositol-1-Phosphate (MIP) Synthase

"Affective theory of mind" is mediated by the ventromedial prefrontal cortex: a lesion study

Shamay-Tsoory S.G. ${ }^{1,2}$, Tomer R. ${ }^{1,2}$, Berger B.D. ${ }^{2}$ and Aharon-Peretz J

${ }^{1}$ Cognitive Neurology Unit. Rambam Medical Center. P.O.B 9602, Haifa; ${ }^{2}$ Dept. of Psychology, University of Haifa

Prefrontal brain damage may result in impaired social behavior, especially when the damage involves the orbitofrontal/ventromedial (VM) prefrontal cortex. It has been previously suggested that deficits in theory of mind (ToM) may account for the aberrant behavior observed in patients with prefrontal lesions. However, inconsistent results have been reported and different regions within the frontal cortex have been associated with ToM impairment. The present study examined the hypothesis that patients with ventromedial frontal lesions are impaired in the affective rather than cognitive facets of ToM. The performance of patients with localized lesions in the prefrontal cortex $(n=26)$ was compared to responses of patients with posterior $(n=13)$ and healthy control subjects $(n=13)$. Three ToM tasks differing in the level of emotional processing involved were used: second-order false belief task, understanding ironic utterances and identifying social 'faux pas'. The results indicated that patients with VM prefrontal lesions were significantly impaired in irony and faux pas but not in second order false belief, as compared to posterior patients and healthy controls. Furthermore, among the patients with the most impaired ToM, lesions in the right VM area were significantly larger than the lesions in either the left VM, right and left dorsolateral regions. The present results highlight the dissociation between the cognitive and affective facets of 'mindreading' processes mediated by the VM cortex.

Keywords: prefrontal lesions, theory of mind, the entromedial prefrontal cortex

\section{Exposing collinear facilitation in the periphery with} attention

Shani R. and Sagi D.

Dept. of Neurobiology, Brain Research, Weizmann Institute of Science, Rehovot 76100

It has been previously shown in psychophysical studies that detection threshold of a Gabor patch, presented at the fixation point, is reduced when flanked by co-aligned high contrast alike stimuli. In the periphery, increased inhibition is found However physiological studies in V1 of cats and monkeys do show increased firing rates for co-aligned stimuli at $4 \div$.

Here we attempt to resolve this apparent conflict. We show that the absence of peripheral facilitation is not a result of improper scaling. We observed that collinear facilitation was reduced or gone as soon as targets were placed outside fixation $(1 \div)$, stil within the fovea. This suggested the involvement of attentional mechanisms in peripheral facilitation. It has been previously shown that lateral interactions are modulated by attention in the fovea. We assessed the role of attention in peripheral surround modulation by directing attention to the flankers in a double task paradigm. Subjects performed a Vernier acuity task on the flankers and a detection task on a Gabor target located in between. Collinear thresholds were lower than orthogonal ones in the double task. When performing only the detection task, collinear and orthogonal thresholds were similar. This result shows that under conditions that force attention to critical parts of the stimulus, facilitation is present at $4 \div$ and further suggests that differences in allocation of attention between fovea and periphery may underlie the lack of peripheral collinear facilitation. The orientation selectivity of this attention-induced facilitation may imply that it is mediated by lateral interactions, as suggested for foveal facilitation.

Keywords: attention, lateral interactions, periphery 
The involvement of PKC in rule learning in the piriform cortex

Shiboleth A. ${ }^{1}$, Brosh I. ${ }^{2}$, Serussi Y. ${ }^{2}$, Barkai E. ${ }^{1}$ and Rosenblum $\mathrm{K}$.

${ }^{1}$ University of Haifa, Center for Brain \& Behavior; ${ }^{2}$ Dept. of Morphology, Faculty of Health Sciences and Zlotowski Center for Neuroscience, Ben Gurion University

We are interested in the molecular mechanisms of olfactory rule learning in the piriform cortex. We have shown previously that post-burst after-hyperpolarization (AHP) and paired-pulse facilitation (PPF) is reduced in pyramidal neurons, days after olfactory rule learning. Assuming that these long-term cellular modifications subserve odor rule learning, we are looking into their possible molecular mechanisms. (See also Cohen et al). $\mathrm{PKC}$ has been implicated in the process of learning and memory formation. Here we study the involvement of PKC in long-term AHP modulation following odor rule learning. The specific PKC inhibitor GF109203x (10 $\mu \mathrm{M})$ caused an increase in AHP amplitude in neurons from trained rats. Consequently, the difference in AHP amplitude between trained and control rats was diminished. Moreover, activation of PKC by OAG (10 $\mu \mathrm{M})$ significantly reduced the AHP in neurons from naive and pseudotrained rats, but not in neurons from trained rats, abolishing the difference between groups. Using sub-cellular fractionation and quantitative immunoblotting analysis, we are examining the role of the different PKC isoenzymes in rule learning in the piriform cortex. Phosphorylation of PKC / $B I I$ on Thr638/641 was decreased by $15 \%$ in the trained group compared with pseudotrained and naive groups $(n=7)$. In the cytosolic fraction, phosphorylation of PKC $\alpha / \beta I I$ on Thr638/641 was reduced in the trained group by $23 \%$ compared to controls $(n=7)$. We conclude that PKC $\alpha / \beta I I$ phosphorylation and subcellular distribution might be involved with PKC-dependent AHP modulation in the piriform cortex of trained rats.

Keywords: PKC, piriform cortex, rule-learning

Modulation of voltage-dependant potassium channel Kv1.1 by SNARE protein SNAP-25

Shilo N Michaelevski I. Chikvashvili D and Lotan I Dept. of Physiology and Pharmacology, Sackler School of Medicine, Tel-Aviv University, 69978 Ramat-Aviv, Israel.

It has been established that presynaptic voltage-gated $\mathrm{K}^{+}$ channels are involved in shaping presynaptic action potentials and thus regulate neurotransimtter release. It has also been established that both $\mathrm{Kv} \alpha 1.1$ channel and its auxiliary subunit KvB1.1 interact directly with the exocytotic apparatus through the SNARE complex protein syntaxin 1A. In our present work we are examining the prospect of a connection between Kval.1 channel and yet another v-SNARE protein - SNAP-25 (25-kDa synaptosome-associated protein). Such possible connection is being characterized in Xenopus oocytes. From the results we have gathered so far, it appears that SNAP-25, like syntaxin-1A, affects the channel currents in a biphasic manner, according to the concentration in which is it applied. However, as opposed to syntaxin's effect, it seems that low SNAP concentrations cause decrease in amplitude of current through the channel, with or without $K v \beta 1.1$, while at high SNAP concentrations there is an increase in the amplitude of the current through the channel, with or without Kv $\beta 1.1$. SNAP-25 does not affect the channel's steady state activation. We are currently studying the effects of SNAP-25 on the steady-state $\mathrm{N}$-type inactivation of the $\mathrm{Kvl} 1.1 / \mathrm{Kv} \beta 1.1$ channel. We are also examining the possibility of a crosstalk between the effect of syntaxin 1A and the effect of SNAP-25

Keywords: $K v \alpha 1.1, \mathrm{Kv} \beta 1.1$, SNAP-25, syntaxin 1A

It may be easier to see two things at the same time! Shneor E and Hochstein S.

Dept. of Neurobiology, Institute of Life Sciences and Neural Computation Center, Hebrew University, Jerusalem

It is well known that subjects can detect rapidly an element that differs greatly from surrounding elements in a single dimension such as color or orientation. With attention spread across the entire array, subjects detect presence or absence of such a target with a response time that does not depend on the number of distractor elements. We now asked what will be the speed and accuracy of detecting 2 such elements simultaneously. Subjects viewed a briefly presented $8 \times 8$ array of pink lines oriented at $55^{\circ}$ (or $60^{\circ}$ ) followed by a masking stimulus after a variable Stimulus-to-mask Onset Asynchrony. On some trials, 1 or 2 of the elements were replaced by a pale green line of the same orientation, a pink line of orientation $35-40^{\circ}$ (or $30^{\circ}$ ), or a line with both these changes. Subjects reported the number of odd lines, and their nature. Surprisingly, we found that subjects were more accurate at detecting targets in arrays with 2 odd elements than arrays with one - despite the requirement of accurately reporting the nature of the change. This result was true for all types of odd elements. In addition, we found that it was easier to report presence of two odd elements - one with an odd color and one with an odd orientation - than to report the presence of one odd element - which differed from the distractor elements both in color and orientation. Analysis of these results as a function of distance between the odd elements suggests that oddity is detected as a Gestalt and that arrays with a pair of targets are perceived as distinct structures.

Transgenic increase in the "readthrough" acetylcholinesterase variant (AChE-R) associates with increased motor activity of FVB/N mice

Shoham S. ${ }^{1}$, Kovalev E. ${ }^{\mathrm{Y}}$, Cohen O. ${ }^{2}$, Yirmiya $\mathrm{R}^{3}$ and Soreq $\mathrm{H}$.

${ }^{1}$ Research Dept., Herzog Hospital, Jerusalem; ${ }^{2}$ The Institute of Life Sciences, Jerusalem; ${ }^{3}$ Dept. of Psychology, The Hebrew

University, Jerusalem

Cholinergic neurotransmission is extensively involved in the function of motor systems. This clearly includes the "synaptic" acetylcholinesterase variant AChE-S, multimeric complexes of which adhere to the synaptic membrane. However, it was unknown whether the monomeric, soluble "readthrough" AChE-R variant also contributes to the regulation of motor activity. To address this issue, open field motor activity was video-photographed in $\operatorname{TgR}(n=8)$ and FVB/N mice $(n=8)$. In addition, locomotor activity of $\operatorname{TgR}(n=10)$ and $F V B / N$ $(n=10)$ mice was telemetrically recorded in the home cage. In an open field, $\mathrm{TgR}$ but not FVB/N mice, displayed frequent episodes of running in circles at a rate of $13 \pm 4$ per min. In the home cage, telemetry of TgR mice revealed abnormally large variabilities in the circadian pattern of motor activity. We employed rabbit anti-hAChE-R and goat anti-choline acetyltransferase (ChAT) antibodies to correlate the anatomica distribution of human (h)AChE-R in the brain of transgenic FVB/N mice (TgR) with their cholinergic nature and their capacity to control locomotor activity. hAChE-R was immunolabeled in cortical and hippocampal neurons, targets of cholinergic neurons. In contrast, cholinergic neurons of the medial septum, diagonal band and striatum did not contain hAChE-R although they displayed intense ChAT staining. An exception was the nucleus basalis magnocellularis (NBM), in which 5\% of ChAT-positive neurons, known to be involved in regulation of cognitive and motor functions displayed $\mathrm{hAChE}-\mathrm{R}$ staining. Our findings demonstrate that alterations in AChE-R expression in cholinergic neurons of the NBM and in target regions of cholinergic neurons may causally contribute to the modulation of motor activity.

Keywords: acetylcholine, acetylcholinesterase, motor behavior, NBM

Genetic and gender factors in physiological, behavioral and neuronal activation responses to diet restriction Shoham S. ${ }^{1}$, Kovalev E. , Marcus EL. ${ }^{1}$, Avraham Y. ${ }^{2}$ and Berry EM.

${ }^{1}$ Research Dept., Herzog Hospital, ${ }^{2}$ Human Nutrition and Metabolism, Hadassah University Hospital, Jerusalem

Human anorexia nervosa (AN) involves self-imposed diet restriction (DR). It is not known how DR may modulate psychiatric phenomena in AN. In light of evidence for contribution of genetic and gender factors to $\mathrm{AN}$, the present study compares responses to DR in the strains of inbred mice, $\mathrm{C} 57 \mathrm{BL}$ and $\mathrm{BALB} / \mathrm{c}$, representing diverse genetic backgrounds focusing on (1) body weight response, (2) behaviora responses: learning (serial maze task), conflict behavior (between hiding and exploration) in the elevated plus maze and emergence paradigms and (3) brain neuronal response assessed by immunohistochemical staining of the immediate early gene product, $c$-FOS. Mice were assigned to groups, receiving ad libitum (AL), or $70 \%$ or $60 \%$ of daily $\mathrm{AL}$ food intake. BALB/c mice displayed greater weight loss in response to DR with greater loss in females than in males. This was associated with DR-induced c-FOS in the arcuate nucleus of $\mathrm{BALB} / \mathrm{c}$ but not of $\mathrm{C} 57 \mathrm{BL}$ mice. Female BALB/c mice did not display DR-induced c-FOS in the dorsomedial and lateral hypothalamus whereas $\mathrm{C} 57 \mathrm{BL}$ did. BALB/c mice displayed greater DR-induced reduction of emotional conflict (increased exploration in novel environments) than did C57BL mice but had no deficit in learning. DR induced c-FOS in hippocampal $\mathrm{CA} 1$ and dentate gyrus of $\mathrm{BALB} / \mathrm{c}$ but not of $\mathrm{C} 57 \mathrm{BL}$ mice. The $\mathrm{BALB} / \mathrm{c}$ strain emerges as a model for $\mathrm{AN}$, in displaying greater changes in emotional behavior and changes in activation of limbic brain regions in response to DR. These findings suggest pathways by which DR may modulate psychiatric phenomena in AN. 
Keywords: diet restriction, anorexia, genetic background, gender differences

Distribution of small-conductance, calcium-activated potassium channel 3 (SK3) in mouse brain Shoham S. ${ }^{1}$, Bronstein-Sitton N. ${ }^{2}$, and Rozen $\mathrm{Y}^{2}{ }^{2}$ ${ }^{T}$ Research Dept., Herzog Hospital, Jerusalem, ${ }^{2}$ Alomone labs, Jerusalem

The calcium influx that occurs during an action potential activates, among others, small-conductance, calcium-activated potassium channels (SK). Since both SK3 mRNA and protein have been identified in rat substantia nigra pars compacta (SNC) and ventral tegmental area (VTA), the SK3 channel has attracted much attention in the context of dopaminergic neuronal function and implications for schizophrenia. However, SK3 has not been mapped in mouse brain. The present study documents SK3 distribution in brain of BALB/c mice. At the forebrain level, SK3 staining was documented in thalamic ventromedial complex and laterodorsal lamina, in the medial habenula, and in the central and corticomedial amygdala. In the mesencephalon, there was staining in the SNC and VTA and in superficial lamina of the superior colliculus. At the pontine level there was staining in the locus coeruleus and parabrachial nucleus. All staining patterns were blocked by pre-incubation of the antibody with the antigen. The SK3 distribution in mice documented in the present study parallels the distribution reported in rats, with the additional presence of SK3 in the amygdala and ventral thalamus. Thus, the present study supports the possibility that SK3 in ventral mesencephalic dopaminergic neurons is ubiquitous in mammalian species and that this localization could be preserved in humans. SK3 distribution in mice suggests possible involvement of SK3 in pathways that modulate sensory information processing. The presence of SK3 in the central amygdala nucleus may suggest that in mice, SK3 also participates in neural pathways for integration of sensory stimuli in visceral-emotional reactions.

Keywords: SK3, calcium, potassium, channels

A system for rapid uncaging in neural tissue using spatially defined patterns

Shoham S. and Wang S. S.-H.

Dept. of Molecular Biology, Princeton University, USA

Two-photon laser scanning microscopy has enabled fast high-resolution imaging deep into brain tissue, which is strongly light scattering. The ongoing development of functional fluorescent probes and high-throughput techniques for dye loading promise an increasing range of applications of multiphoton optical methods in neuroscience.

The use of light-sensitive "caged" compounds complements two-photon microscopy by providing a means of optically manipulating biochemical signals. We are developing a flexible new system that allows controlled photo-release of caged neurotransmitters in defined spatial and temporal patterns with submicron and submillisecond resolution. Our system relies on the steering of an ultraviolet laser beam in two dimensions using acousto-optical devices made from $\mathrm{TeO}_{2}$. The ultraviolet light pattern is projected into the same plane scanned by a custom-made two-photon microscope. The beam can be steered to up to 100,000 locations per second. We are currently beginning to apply the system to neural systems.

Keywords: two-photon microscopy, uncaging, slice physiology, optics

Functional Ras antagonist inhibits the increase in brain Ras-GTP induced by closed head injury and exerts neuroprotection

Shohami E. ${ }^{1}$, Yatsiv I ${ }^{1,2}$, Alexandrovich A ${ }^{1}$, Haklai R. ${ }^{3}$, Elad-Sfadia G. $^{3}$, Grossman R. ${ }^{4}$, Biegon A. and Kloog Y. ${ }^{3}$ ${ }^{1}$ Dept. of Pharmacology. School of Pharmacy and ${ }^{2}$ Pediatric Intensive Care Unit, Hadassah Medical Center, The Hebrew University, Jerusalem, ${ }^{3}$ Neurobiochemistry, Tel Aviv University, ${ }^{4}$ Neurosurgery, Sheba Hospital, and ${ }^{5}$ Lawerence Berkeley National Laboratory, Berkeley CA, USA

Ras proteins are involved in receptor-mediated signaling pathways, including those regulating cell death and survival Ras antagonists may therefore be protective when these pathways are activated. S-trans, transfarnesylthiosalicylic acid (FTS), a Ras inhibitor, acts primarily on the active, GTP-bound, form of Ras. This study investigates whether: 1) closed head injury (CHI) activates Ras; 2) FTS interferes with Ras activation; 3) FTS affects functional outcome, NMDA receptor function and lesion volume after $\mathrm{CHI}$. Mice were subjected to $\mathrm{CHI}$ or sham operation, treated with vehicle or FTS $(5 \mathrm{mg} / \mathrm{kg})$, and sacrificed $2 \mathrm{~h}, 24 \mathrm{~h}$ or $7 \mathrm{~d}$ after CHI. Total Ras, Ras-GTP, ERK and Ras-dependent active phospho-ERK were determined using specific $A b$. Neurological deficits were assessed by a Neurological Severity Score (NSS) at $1 \mathrm{~h}-7 \mathrm{~d}$ post $\mathrm{CHI}$. Recovery was defined by $\triangle \mathrm{NSS}(\mathrm{t})=\mathrm{NSS}(\mathrm{lh})-\mathrm{NSS}(\mathrm{t})$. NMDA receptor binding and lesion volume were measured by autoradiography and morphometry of brain sections, respectively. Ras-GTP was significantly increased in the contused hemisphere at $2 \mathrm{~h}$ post-CHI and this increase was inhibited by FTS and by the NMDA antagonist MK-801. Total Ras was not affected and Ras-GTP levels returned to baseline by $24 \mathrm{~h}$ post $\mathrm{CHI}$. Both drugs reduced phospho-ERK levels after CHI. $\triangle$ NSS and neurological deficits were significantly improved $(p<0.0001,60 \%)$ by FTS, which also prevented the CHI-induced reduction in NMDA receptor binding in cortical, striatal and hippocampal regions and produced a trend towards smaller lesions. We propose that Ras activation after $\mathrm{CHI}$ contributes to neuronal cell death and its suppression by FTS has a long-lasting beneficial effect

Keywords: traumatic brain injury, Ras-GTP, S-trans transfarnesylthiosalicylic acid (FTS), NMDA receptors, neuroprotection

Activation of Matrix metalloproteinases by TNF- $\alpha$ in astrocytes in vitro: a model for brain injury Shoshany $\mathrm{Y}^{1}$, Shohami $\mathrm{E}^{1}$. Brenner $\mathrm{T}^{2}$ and Reich $\mathrm{R}$. Dept. of Pharmacology, Hebrew University of Jerusalem: ${ }^{2}$ Dept. of Neurology, Hadassah University Hospital, Jerusalem Following head trauma several processes take place including inflammation, neurotransmitter release and cell death. Cytokine action followed by specific gene's activation play a key role in the pathophysiology of brain injury. Among the earliest events (hours) is the disruption of the blood-brain barrier (BBB) Matrix metalloproteinases (MMP) are involved in the disruption of the blood-brain barrier as well in the repair process, taking place in the injured brain. TNF $\alpha$, a cytokine upregulated within $1 \mathrm{~h}$ after trauma was shown to play a major role in the processes leading to BBB disruption. In experimental model of closed head injury, administration of dexanabinol prevented TNF $\alpha$ mediated BBB breaching. In the present study, we undertook to investigate the role of TNF $\alpha$ in induction of MMP secretion and dexanabinol effect in astrocytes in culture, as a model of brain damage

Our results indicate that TNF $\alpha$, dose dependently, induces the secretion of MMP-2 and MMP-9 in astrocytes culture Furthermore dexanabinol prevented the elevation of both MMPs. RT-PCR experiments indicate that the site of action of dexanabinol is at the transcriptional level. Stimulation of astrocytes with LPS led to elevation of TNF $\alpha$ activity that was inhibited by dexanabinol. The inhibitory effect of the drug was on the release of TNF $\alpha$ from membranal site by TACE RT-PCR analysis showed no significant effect on TNFo mRNA whereas TACE mRNA levels were reduced significantly. To our knowledge, this is the first demonstration of a direct effect of dexanabinol on TNF $\alpha$ production/release via inhibition of TACE production in astrocytes.

Keywords: Tumor necrosis factor (TNF $\alpha$ ), matrix metalloproteinases (MMP), dexanabinol, cultured astrocytes, blood-brain barrier

\section{Synaptic dynamics determine the functional correlations between neocortical neurons in excited slices}

Silberberg G. ${ }^{1}$, Wu C.Z.' and Markram H.

${ }^{1}$ Brain \& Mind Institute, EPFL. Lausanne, Switzerland: ${ }^{2}$ Dept.

of Neurobiology, Weizmann Institute of Science, Rehovot

Stereotypical microcircuits have long been recognized as computational modules of neocortical function, but the principles that determine the emergence of activity patterns within these microcircuits are unknown. We obtained multiple simultaneous whole-cell voltage recordings from neurons in neocortical slices. A cross-correlation analysis of subthreshold membrane voltage between different types of neurons revealed the emergence of characteristic functional relationships between neurons when the slice was activated by an excitant extracellular solution. The functional relationship between neuronal pairs was correlated to the nature of the excitatory synaptic input to these neurons, specifically to the governing synaptic dynamics. In simulations, differences in cross-correlation delays emerged when the excitatory discharge was patterned and deviated from purely random spike activity In this study we show that synaptic dynamics determine the relative timing of activity of different neurons and thereby impose stereotypical response characteristics in the neocortical microcircuit as a function of the excitatory activity pattern.

Keywords: neocortical microcircuits, synaptic dynamics.

multineuron recordings, subthreshold correlations 
Perception of happy and sad facial expressions in chronic schizophrenia: Evidence for two evaluative systems Silver $\mathrm{H}^{1,2}$, Shlomo N ${ }^{3}$, Travis T. ${ }^{4}$ and Gur R. 'Sha' ar Menashe MHC and Technion; ' Mazra MHC, University of Pennsylvania

Background: Schizophrenia patients have impaired perception of emotional expressions, but it is not clear whether this is part of a generalized deficit in cognitive function. Aim: To test for existence of emotion-specific deficits by studying the effects of valence on recognition of facial emotional expressions. Methods: 24 male subjects suffering from chronic schizophrenia were examined with two tests of perception of emotion: the Penn Emotion Acuity Test (PEAT 40) and the Emotion Differentiation Task (EMODIFF). Clinical state was assessed with the SANS and SAPS scales, visual memory with the Benton Visual Retention Test (BVRT) and motor function with the finger tapping test. Results: Identification of happy facial expressions showed significant negative correlation with age, cumulated time in hospital and length of current hospitalization; positive correlations were found with visual retention and finger tapping scores. Identification of sad facial expressions showed significant correlation only with cumulated time in hospital while identification of neutral facial expressions showed no significant correlations. Discrimination between degrees of happy but not sad facial expression, showed a positive correlation with negative symptoms. Conclusion: Perception of happy and sad emotion relates differently to significant illness parameters. This differentiability supports the existence of an emotion-specific deficit in perception of emotions in schizophrenia and of separate channels for processing positive and negative emotions.

Keywords: emotions, schizophrenia, valence, cognitive

Non-auditory brain structures involved in processing auditory linguistic material

Sinai A and Pratt $\mathrm{H}$.

Evoked Potentials Laboratory, Technion-Israel Institute of Technology, Haifa 32000

The purpose of the study was to localize non-auditory cortical areas activated during first and second language processing. Brain potentials were recorded from 17 normal hearing, right handed, native Hebrew (first language) speakers that also speak English (second language). Subjects performed a lexical decision task with speech stimuli from both languages. Brain sources of activity were estimated by calculating current densities from the scalp distribution of the ERPs, using low-resolution electromagnetic tomography analysis (LORETA). Current densities in brain voxels (cubicles of 7 $\mathrm{mm}$ spatial resolution) were compared with pre stimulus baseline by non-parametric t-tests adjusted for multiple comparisons. The time course of activity in each Brodman area (BA) was plotted separately for the right and left hemisphere. Brain regions outside the traditional auditory and language-related regions demonstrated different temporal patterns of activity that varied with the type of stimulus. These regions included, among others, frontal lobe motor regions, pre-frontal associative region, cingulate gyrus and pre-cuneus. The structure-specific spatio-temporal pattern of activity is compatible with the idea that language processing occurs in spread out neural networks involved with processing different aspects of language.

Keywords: hearing, language, functional imaging,

Event-Related Potentials

The neural representation of odor pleasantness and intensity in the human brain

Sobel N. and Anderson K.N.

Helen Wills Neuroscience Institute, University of California at Berkeley, Berkeley, CA, 94720 USA

Imaging studies have shown increased amygdaloid complex (AC) activation in response to aversive odors. Odor quality is dependent on odor concentration (intensity) and odor hedonics (pleasantness/unpleasantness). Here we dissociate these dimensions, and ask whether $\mathrm{AC}$ activity reflects odor hedonics, intensity, or both. An olfactometer generated 5 stimuli; 1) PH - pleasant high intensity, 2) PL - pleasant low intensity, 3) UH - unpleasant high intensity, 4) UL - unpleasant low intensity, 5) clcan air. 16 young healthy subjects participated in an event-related $\mathrm{MMR} I$ study at $3 \mathrm{~T}$ (T2* spiral $\mathrm{TE}=30, \mathrm{TR}=1 \mathrm{~s}, 64 \times 64$ FOV, 17 slices, slice thickness $=$ $4 \mathrm{~mm}$, stimulus ISI $=20 \mathrm{~s}$, stimulus repetition $=30$ ). A first-pass analysis using SPM99 to compare for regions of increased activity related to odor intensity regardless of pleasantness revealed a pronounced locus of activity in the $\mathrm{AC}(\mathrm{P}<0.001)$. In contrast, an analysis comparing for increased activity related to the unpleasantness of an odorant regardless of intensity, revealed a locus of activity in the orbitofrontal gyri $(\mathrm{P}<0.001)$. In-depth ROI analysis corroborated these findings, demonstrating an increase in signal change in the $\mathrm{AC}$ in response to increases in intensity, not unpleasantness, of an odor Activity in the $\mathrm{AC}$ was significantly correlated with individual intensity estimates $(\mathrm{r}=0.35, \mathrm{p}=0.001)$ but not with pleasantness estimates $(r=0.04, p=0.7)$. These findings suggest that the AC may be encoding more of the immediate physical dimensions of a stimulus (intensity) rather than the later "psychological dimensions" of the stimulus (pleasantness).

Keywords: olfaction; fMRI; amygdala

The molecular neurobiology basis of physiological stress responses

Soreq $\mathrm{H}$.

Institute of Life Sciences, The Hebrew University of Jerusalem

Physiological symptoms reminiscent of post-traumatic stress disorder (PTSD) occur following exposure to anticholinesterases, however, the origin of this phenomenon is unknown. Recently, we found a dominant activating polymorphism in the ACHE promoter, carried by over $5.0 \%$ of the Israeli and compared with $0.5 \%$ of the North American population. This polymorphism is associated with acetylcholinesterase (AChE) overproduction and hypersensitivity to cholinergic agonists and antagonists (Shapira et al., Hum. Mol. Genetics, 9:1273-1281 [2000]). Its consequences could be attributed to AChE's hydrolytic activity, the complexity of ACHE gene regulation and/or the "non-classical" AChE activities (Soreq \& Seidman, Nature Neurosci. Rev. 2:294-302. [2001]). There are three 3' splicing options for AChE mRNA, and 3 corresponding AChE variants with different C-terminal peptides, multimeric assembly and non-classical function(s). Of these, the normally rare "readthrough" variant, AChE-R, accumulates following stressful stimuli and under acetylcholinesterases (Kaufer et al., Nature 393:373-377 [1998]) or under neuromuscular diseases (e.g. myasthenia gravis) (Brenner et al., FASEB J., in press [2002]). The AChE-R mRNA, usually located in neuronal cell bodies, translocates to dendritic processes following stress This rapid yet long-lasting change may suppress the initial insult, but leads to adverse consequences under long-term conditions (Meshorer et al., Science 295:508-512 [2002]) AChE-R involvement in these adverse symptoms was proven by using partially 2'-oxymethylated oligonucleotides inducing AChE-R mRNA destruction (Shohami et al., J. Mol. Med. $78: 228-236[2000])$. The variant selectivity of these antisense agents, currently tested in clinical trials, prevents the stress-induced physiological impairments while protecting cholinergic neurotransmission by maintaining the synaptic AChE.

Keywords: stress, pharmacogenomics, mRNA translocation, acctylcholinesterase

On line confocal imaging of processes underlying the dedifferentiation of an axonal segment into a motile growth cone after axotomy

Spira M.E., Erez H., Khoutorsky A. and Prager-Khoutorsky M. Dept. of Neurobiology, Life Sciences Institute, The Hebrew University of Jerusalem, Jerusalem

Regeneration of neuron after mechanical injury requires structural and functional dedifferentiation of the cut axonal end into a motile growth cone (GC). Here we analyzed the events leading to the formation of a growth cone (GC) and examined the mechanisms that set the dedifferentiation process into motion.

To that end we fluorescently labeled actin by chimeric EGFP-actin, microtubules (MTs) by tetramethylrhodamine conjugated tubulin, and vesicles by the styril dye $\mathrm{RH}-237$, in primary cultures of identified Aplysia neurons.

Confocal imaging revealed that: (a) axotomy triggers a wave of MTs depolymerization. The depolymerizing wave stops at a point $100-150 \mu \mathrm{m}$ from the cut axonal end, forming a transition zone (TZ) between the depolymerized segment and a proximal segment in which the MTs maintain normal structure. (b) In parallel, actin reach adhesion plaques disappear only to reform minutes later. (c) Anterogradlly transported vesicles are trapped within the TZ. Thereafter, (d) actin bundles assemble along the axons perimeters surrounding the vesicles trap. Once the above-described structure is formed an actin reach GC lamellipodium, supported y radial polymerization of MTs, begins to extend.

Axotomy in the presence of nacodasol prevents the restructuring of the depolymeryzed MTs and the trapping of vesicles, but does not affect the initial extension of a GC's lamellipodium. In jusplakinolid the MTs based vesicles trap is 
formed, but the extension of a GC's lamellipodium is blocked. Calpeptin totally inhibits the restructuring of the axon (Oren et al., this meeting).

We conclude that a common calpeptin sensitive event triggers the dedifferentiation process.

Keywords: axotomy, growth cone, regeneration, calpain, microtubules

Color adaptation models and their predictions to color induction effects, color constancy and color contrast Spitzer H. and Barkan Y.

Dept. of Biomedical Engineering, Faculty of Engineering, Tel Aviv University, Tel Aviv

A new biological model and algorithm for color adaptations, which includes the color constancy (first order) and color contrast modulation (second order), is presented. (Color constancy (CC) is a psychophysical phenomenon in which a system has a partial capability to discount the chromaticity of the illumination). The goal of the model was to achieve computational psychophysical predictions to different color appearance effects such as color constancy, color contrast and different induction effects. The model predicts the above human visual performance, including the modulation of perceived color due to surrounding color. It predicts also the dual effects of enhancement and the suppression of the central perceived contrats due to the relation between the central and surround contrasts. The model is based on the properties of retinal ganglion cells (opponent cells) and cortical cells (double opponent cells), as well as on chromatic adaptation mechanisms in these double opponent color-coded cells: remote chromatic adaptation. The suggested color adaptation mechanisms are modeled as gain control mechanisms based on the "curve-shifting" effect. This effect is the transition from one response curve to another, due to a change in the light intensity (or color, or contrast) of the local receptive field and its remote area, in order to obtain a higher gain in the new color or contrast. The simulations calculated also on real images. The results indicate that the contribution of adaptation mechanisms to color constancy and color contrast are significant, robust, and enables color enhancement, color contrast enhancement and color constancy of still and video images.

Keywords: visual system, color vision, adaptation, remote effects

Omega-3 fatty acid treatment of depressive breakthrough during unipolar maintenance

Stahl Z., Nemets B. and Belmaker RH.

Ministry of Health Mental Health Center, Faculty of Health

Sciences, Ben Gurion University of the Negev, Beer-Sheva

Objective: Studies have reported that countries with high intake of fish oil have low rates of depression. We studied a specific omega-3 fatty acid, the ethyl ester of eicosapentaenoic acid (E-EPA) as an adjunct to antidepressant treatment for breakthrough depression in recurrent unipolar patients on maintenance therapy.

Methods: Design was four-week parallel group double-blind add-on to ongoing antidepressant therapy. Twenty patients participated, seventeen females and three males, all with diagnosis of current major depression.

Results: Highly significant benefits were found by week 3 of treatment for eicosapentaenoic acid compared to placebo.

Conclusions: It is not possible to distinguish whether eicosapentaenoic acid augments antidepressant action in the manner of lithium or has independent antidepressant properties of its own.

Keywords: omega-3, depression, antidepressant

Gustatory thalamus: Where does it connect to?

Stehberg J. and Dudai Y

Dept. of Neurobiology, Weizmann Institute of Science, Rehovot 76100

The role of the gustatory thalamus in gustatory function has been controversial, due mainly to the fact that unlike other senses, gustatory projections from the parabrachial nucleus (PBN) go either directly to the cortical taste area or travel via the thalamus. A combination of retrograde (fluorogold) and anterograde (BDA and FDA) neuronal tracers was used to map the afferents and efferents of the gustatory thalamus Ventroposteromedial parvicellular nucleus. Anterograde and retrograde tracers were injected into the insular cortex and $\mathrm{PBN}$. We found that the PBN afferents to the thalamus do not colocalize with CGRP and that the CGRP axons have a non-overlapping more ventral localization from the PBN axons. Thalamic neurons projecting to the cortex localize indistinctively along the nucleus, covering areas that receive input from the PBN, CGRP and areas that do not receive input from neither of the above areas. Corticothalamic projections also do not show a clear mapping, terminals being found all over the nucleus. We were also able to determine the cellular location of the PBN synaptic terminals in the thalamic neurons. In search for a functional topographic map of the thalamus, we combine thalamic retrograde labeling from the cortex and pCREB immunohistochemistry as an activity marker. We test whether there is a distinctive activation of the thalamocortical projections when the rat drinks novel taste vs. a familiar one Supported by the Minerva Foundation and the Human Frontiers Science Program Organization.

Keywords: thalamus, taste, cortex, pCREB, tracers.

Role of $\mathbf{G}_{\beta \gamma}$ in the regulation of adenylyl cyclase activity following acute and chronic receptor activation Steiner D., Schallmach E., Butovsky E., Nevo I., Saya D. and Vogel Z.

Dept. of Neurobiology, Weizmann Institute of Science, 76100 Rehovot

Acute activation of opiate (and other $G_{i / 0}$ ) receptors inhibits adenylyl cyclase (AC) activity, while prolonged activation leads to an increase in $\mathrm{AC}$ activity, known as AC sensitization or superactivation. This phenomenon has been proposed to play a role in opiate addiction and has been shown for AC types I, V, VI and VIII, but not for AC-II, IV or VII demonstrating that superactivation is isozyme-specific. To investigate the roles of $G_{\beta \gamma}$ and $G_{\alpha i}$ in $A C$ regulation, we produced several point mutations in $A C-V$ and $A C-I$. We found that a mutation in the $C_{1 a}$ region of AC-V (F481Y) and of AC-I (F314Y) led to reduced inhibition by acute agonist treatment and to the loss of chronic agonist induced superactivation, and in parallel to loss of $A C$ sensitivity to $G_{\beta y}$ dimers and to constitutively active $\mathrm{G}_{\mathrm{\alpha}}$, (demonstrating that this amino acid in $\mathrm{C}_{1 \mathrm{a}}$ plays an important role in the regulation of $\mathrm{AC}$ activity and its interaction with $G$ protein subunits. To distinguish between the roles of $G_{\alpha i}$ and $G_{\beta \gamma}$, three other mutations in $C_{1 a}$ region (shown previously to reduce affinity of $G_{\alpha i}$ to $A C$ ) were prepared. Indeed the activity of these mutants was not inhibited by constitutively active $G_{\alpha i}$ (compared with $70 \%$ inhibition of AC-V wild-type). On the other hand, these mutants behaved identically to wild-type $A C-V$ with respect to $G_{\alpha s}$ stimulation and $\beta \gamma$ inhibition and showed normal opiate acute inhibition and chronic induced superactivation. These results suggest that the inhibition of $A C$ by $G_{\beta \gamma}$, and not by $G_{\alpha i}$, is essential for the superactivation process

Supported by NIDA and the US-Israel Binational Science Foundation.

Keywords: $\mathrm{G}_{\mathrm{i} / 0}$-coupled receptor; adenylyl cyclase; opiates

Central pathways between sacrocaudal afferents and locomotor circuits in the spinal cord of the newborn rat Strauss I. and Lev-Tov A.

Dept. of Anatomy \& Cell Biology, The Hebrew University

School of Medicine, Jerusalem

The central pathways mediating sacrocaudal afferent (SCA) activation of locomotor circuits in the lumbar spinal cord were studied in isolated spinal cords of neonatal rats. The lumbar rhythm induced by SCA stimulation was abolished after blocking synaptic transmission in the sacrococcygeal (SC) cord and restored when the synaptic block of specific SC segments was temporarily alleviated. Thus, synaptic activation of SC relay neurons is required for generation of locomotor activity by SCA stimulation.

The transmission across these relays required activation of non-NMDA excitatory amino acid receptors, and it was not impaired in the presence of specific antagonists of NMDA receptors and $\alpha 1$ and $\alpha 2$ adrenoceptors

Midsagittal splitting experiments of parts of the SC and lumbar cord revealed that crossed and uncrossed ascending/propriospinal pathways are coactivated by SCA stimulation. These pathways ascend onto the lumbar cord through the lateral, ventrolateral, and ventral funiculi, because the lumbar rhythm could be completely abolished only following a bilateral transection of all of these funiculi. Further studies are required to better understand the SCA system and the pathways associated with it, and unveil its potential use in facilitating stepping in patients with spinal cord injury.

Keywords: pattern generation, spinal cord, propriospinal

pathways 
A novel thiol antioxidant that can cross the blood brain barrier protects dopaminergic neurons in the three experimental models of Parkinson's disease Stroomza M. ${ }_{2}^{1}$ Atlas $D^{2}{ }^{2}$, Gilgun-sherki Y. ${ }^{1}$, Livnat A. ${ }^{3}$, Offen $\mathrm{D}^{1}$ and Melamed $\mathrm{E}^{1}$

${ }^{1}$ Felsenstein Medical Research Center, Dept. of Neurology Rabin Medical Center, Tel Aviv University, Sackler School of Medicine, Petah-Tikva 49100; ${ }^{2}$ Institute of Life Sciences, Edmond Safra Campus Givat Ram, Jerusalem, $91904 ;^{3}$ Novia Pharmaceuticals Ltd. P.O.Box 10209 Tel Aviv 61101; doffen@ipost.tau.ac.il

Oxidative stress is believed to play a crucial role in the degeneration of nigral dopaminergic neurons in Parkinson's disease. Dopamine (DA) and related catecholamines were suggested to contribute to the selective degeneration of dopaminergic neurons via the formation of free radicals. Most currently available antioxidants cannot readily penetrate the blood-brain barrier. We have synthesized a novel glutathione-like low molecular thiol antioxidant (AD4) capable of penetrating the brain following systemic administration. We demonstrated that in vitro, it prevents, neuronal cell death induced by a series of parkinsonian-related neurotoxins. Furthermore this new agent demonstrated significant neuroprotection in three experimental models. I) AD4 inhibited the rotational behavior induced by apomorphine in rats injected unilaterally into the nigra with 6-hydroxydopamine. II) Rats treated with rotenone, a complex I inhibitor, $(5 \mathrm{mg} / \mathrm{kg}$ for 28 days) show loss of dopaminergic neurons in the substantia nigra mimicking parkinsonian characteristics. AD4 attenuated the loss of the dopaminergic neurons as indicated by immunostaining with tyrosine hydroxylase antibodies. III) AD4 was also effective in preventing the reduction in striatal dopamine levels in mice treated with MPTP $(25 \mathrm{mg} / \mathrm{kg} \mathrm{x} 4$, i.p.). These results suggest that AD4 could become a potential new neuroprotective drug used to slow down nigral neuronal degeneration and illness progression in patients with Parkinson's disease

Keywords: Parkinson's disease, dopamine, AD4, MPTP, 6-OHDA, rotenone

The association of aggressive behavior in schizophrenia with the low enzyme activity COMT polymorphism Strous RD. ${ }^{1}$, Nolan K. ${ }^{2}$, Lapidus R. ${ }^{1}$, Kotler M. ${ }^{1}$, Saito T. ${ }^{3}$, Lachman $\mathrm{HM}^{3}$.

${ }^{I}$ Beer Yaakov Mental Health Center, Sackler School of Medicine, Tel Aviv University; ${ }^{2}$ Nathan S. Kline Institute for Psychiatric Research, Orangeburg, New York; ${ }^{3}$ Dept. of Psychiatry and Behavioral Sciences Albert Einstein College of Medicine, Bronx, New York

Increased violent behavior in schizophrenic patients may be associated with a polymorphism at codon 158 of the catechol O-methyltransferase (COMT) gene that encodes a low enzyme activity variant. While this finding has been replicated by one group, others have shown discrepant findings which may be due to different diagnostic criteria used to assess violent behavior, or considering the complexity of aggressive behavior, either type I and/or type II statistical errors Consequently, additional studies are needed. In this study, we assess 122 patients with schizophrenia for violent behavio using the Lifetime History of Aggression (LHA) scale, an 11-item questionnaire that is subdivided into Aggression, Self Directed Aggression, and Consequences/Antisocial Behavior subscales. DNA was genotyped for the COMT 158 polymorphism, as well as a functional polymorphism in the monoamine oxidase A gene (MAOA) promoter. Similar to previous findings reported by our group, a statistically significant association was found between violent behavior in schizophrenia and the COMT 158 polymorphism. Mean LHA scores were higher in subjects homozygous for the low enzyme activity COMT variant, $158 \mathrm{Met}(\mathrm{p}=0.005)$. Analysis of the major LHA subscales revealed that the association with 158 Met was due to high scores on the Aggression, and Self Directed Aggression subscales, but not the Consequences/Antisocial Behavior subscale. No significant association was detected for the MAOA gene alone. These observations further suggest that COMT is a modifying gene that plays a role in determining interindividual variability in the proclivity for outward and self directed violent behavior found in a subgroup of schizophrenic patients.

Keywords: schizophrenia, aggression, COMT, polymorphism
Neuroanatomical and immunohistochemical characterization of the brainstem locus for barbiturate-evoked general anesthesia Sukhotinsky I. and Devor M.

Dept. of Cell and Animal Biology, Hebrew University of Jerusalem, Jerusalem

Microinjection of minute quantities of pentobarbital into a restricted region of rat mesopontine tegmentum can induce a state of general anesthesia (Devor and Zalkind, Pain 2001). We have begun to investigate the anatomical connections and histochemical signature of this mesopontine tegmental anesthesia area (MPTA) using anterograde and retrograde tracers and immunocytochemistry. MPTA has bilateral ascending and descending efferent projections to various forebrain and brainstem structures including the caudoputamen, hypothalamic and subthalamic structures intra-laminar nuclei of the thalamus and widespread areas of the mesencephalic, pontine and medullary reticular formation Descending spinal projections reach as far as the sacral segments. MPTA receives bilateral afferent projections from restricted areas of the diencephalon, and the reticular formation. The most important candidate receptor for barbiturates is a modulatory site on the GABAA receptor (GABAA-R), which augments cellular response to GABA. To determine if GABAA-Rs are present on MPTA neurons, serial brainstem sections were immunolabeled with antibodies to the alphal subunit of the GABAA-R. This is the most common GABAA-R alpha subunit in the CNS. Labeled neuronal cell bodies were scattered throughout the MPTA region with dendrites stained to some extent, and heavy staining in the neuropil. A significant proportion of neurons in the MPTA were immunopositive for GABA. Cell bodies and variously orientated labeled fibers were scattered throughout the MPTA region, with considerable staining in the neuropil Immunocytochemical characterization of MPTA cells, together with their connectivity, contributes to the understanding of brainstem circuitry controlling alert behavior

Keywords: general anesthesia, central pain mechanisms

Further studies on the octopus fetch movement Sumbre G. ${ }^{1}$, Flash T. ${ }^{2}$ and Hochner B.

${ }^{T}$ Dept. of Neurobiology, Institute of Life Sciences and Center for Neural Computation, Hebrew University of Jerusalem. Jerusalem 91904; ${ }^{2}$ Dept. of Applied Mathematics, Weizmann Institute of Science, Rehovot 76100

When an octopus reaches towards an object, it forms a bend at the base of the arm which is propagated towards the tip. Interestingly, during fetching, when an end-point motion control strategy should be used in order to bring the object to a specific point (the mouth), a totally different type of movement is performed. In this case, the octopus grips an object, anywhere along its arm, and brings it to the mouth. A 3D kinematic analysis has revealed the following characteristics An articulated-like structure is formed by creating 3 joints along the arm, in a definite spatiotemporal order. In a given movement, the length of the two proximal segments is similar. The joints rotate along a single plain, to accurately bring the end point to the target (mouth). We conclude that the octopus simplifies the motor control of its arm during goal-directed end-point movements by utilizing an adjustable articulated-like structure. This, thereby, drastically reduces the number of degrees of freedom from virtually infinite to only three allowing the octopus to achieve a high level of accuracy. In contrast to arm extensions, where we showed that the motor program is confined to the peripheral nervous system (Science (2001) 293:1845-1848), preliminary lesion experiments show that the fetching movement is controlled by higher motor centers in the brain

Supported by the Israel Science Foundation.

Keywords: cephalopods, motor control, flexible structure, kinematics.

Temporal encoding of horizontal object position by the rat whiskers: recordings from the trigeminal ganglion Szwed M., Bagdasarian K. and Ahissar E.

Dept. of Neurobiology. Weizmann Institute of Science, Rehovot 76100

We investigated how neurons in the trigeminal ganglion of anesthetized rats respond to whisking against objects inserted at different horizontal positions along the anterior-posterior axis. Rhythmic 5 and $8 \mathrm{~Hz}$. whisker movements similar to spontaneous whisking were induced by electrical stimulation of the peripheral buccal branch of the facial motor nerve ("electrical whisking"). A vertical pin-shaped object was placed inside the whisking field at different horizontal 
distances from the whisker resting position. We recorded single- and multi- unit responses and captured video-images of the whisker at $1 \mathrm{~ms}$ resolution. This allowed us to correlate the moment when the whisker touched the object with neural events

We divided single-unit responses into 1) "touch cells" ( $\mathrm{n}=30)$ that responded to the various aspects of whisker - object contact 2) "whisking cells" (n=29) that responded to whisking and 4) "high threshold cells" $(n=21)$ that responded only to very rapid mechanical deflections.

When a whisker protracts, more posterior objects are contacted earlier, and more anterior - later. We found that for all "object detectors" the timing of the first spike occurring after protraction began was tightly correlated with the time of whisker-object contact. We conclude that horizontal position of object is temporally encoded in the responses of these "object detectors" such that firing later relative to protraction initiation signifies more anterior object position. Also, horizontal object position was sometimes encoded be decreasing spike-counts. This coding, however, was less consistent than the latency coding.

Supported by ISF grant 377/02-1

Keywords: object localization, neural code, sensory encoding

High Pressure and $\left[\mathrm{Ca}^{2+}\right]_{0}$ Modulation of Dynamic

Properties of a Cortico-hippocampal Synapse

Talpalar A.E. ${ }^{1,2}$ and Grossman Y.

${ }^{I}$ Dept. Physiology, Faculty of Health Sciences and Zlotowski Center for Neuroscience, Ben-Gurion University of the Negev, Beer-Sheva; ${ }^{2}$ Depts. Neurophysiology, Kaye College of

Education, Beer-Sheva

Hyperbaric environment (>1.5-2 MPa) induce abnormal activity of the nervous system termed high-pressure nervous syndrome (HPNS) that is characterized by cognitive and motor impairments. The neocortex and the hippocampus are involved in this disorder. The medial perforant path (MPP) synapse onto the granule cells of the dentate gyrus is the main path connecting these structures. Cellular effects of high-pressure of helium were studied using electrophysiological recordings in rat corticohippocampal slices at $30^{\circ} \mathrm{C}$. High pressure $(10.1$ MPa) suppressed single MPP field-EPSP (fEPSP) slope by 55 $\pm 7 \%(\mathrm{n}=9)$ while fEPSP's decay time and delay were prolonged. Amplitude, slope and duration of MPP input volley were reduced by 32,26 and $20 \%$ respectively at $10.1 \mathrm{MPa}$ $(n=5)$. Pressure reduced paired-pulse depression (PPD) observed at short inter-stimulus-intervals (ISI), and increased paired-pulse facilitation (PPF) at ISIs $40-120 \mathrm{~ms}$ (from 5-20 $\%)$. Pressure increased synaptic frequency-dependent depression (FDD) at $25-50 \mathrm{~Hz}$. Reduction of $\left[\mathrm{Ca}^{2+}\right]_{0}$ from 2 to $1 \mathrm{mM}$ under normobaric conditions mimicked high-pressure effects on single fEPSPs' slopes (depressed by $51 \pm 8 \%, n=7$ ) and partially reproduced high-pressure increase in PPF $(n=5)$. In contrast, low $\left[\mathrm{Ca}^{2+}\right]_{0}$ induced frequency-dependent potentiation (FDP) instead of FDD observed at pressure $(n=5)$. The data indicate that high-pressure effects on single and paired synaptic responses are induced by impaired $\mathrm{Ca}$-entry at presynaptic terminals. However, the responses at high frequency suggest that additional release mechanisms such as vesicle replenishment, priming or docking at synaptic sites are impaired by high pressure.

Keywords: hyperbaric pressure, HPNS, field potentials, paired-pulse facilitation, frequency-dependent depression, presynaptic $\mathrm{Ca}^{2+}$ influx, perforant path, dentate gyrus

mGSTM5 KO mice as a potential model for brain studies Tchaikovskaya $\mathrm{T}^{1}$, Fraifeld $\mathrm{V}^{2}$, Asraf $\mathrm{I}^{2}$, Sagi $\mathrm{O}^{2}$, Wolfson $M^{2}$ and Listowsky $\bar{I}$

${ }^{I}$ Albert Einstein College of Medicine of Yeshiva University.

Bronx, NY; ${ }^{2}$ Ben-Gurion University of the Negev, Beer-Sheva

Glutathione S-transferases (GSTs) are members of a superfamily of detoxification enzymes. Recent findings suggest some GSTs may have some other functions as well. mGSTM5 (ortholog of hGSTM3) could be one of those with distinct functions. We found that this cytosolic isoform is abundant in testis, well represented in brain and barely expressed in other organs. Peptide sequence specific mGSTM5 and hGSTM3 antisera were used to localize this isoform in paraffin sections of mouse and human brains by immunoperoxidase staining. mGSTM5 was found to be unevenly distributed in differen regions of mouse brain: strong positive staining was observed in perikarions and processes of Purkinje cells in cerebellum neurons in anterior thalamus and forebrain and neuronal processes in cortex. Analysis of sections from patients who were diagnosted with Alzheimer's disease, revealed an accumulation of this isoform in neurofibrillary tangles and neuritis in hippocampus. These findings indicate that
hGSTM3/mGSTM5 may play a specific role in brain. To further investigate the possible implications of this isoform on organism function and pathology, we developed the GSTM5 knockout mice. GSTM5 KO mice did not display any abnormal phenotype under usual conditions. However, the level of expression of some stress-related genes differed in $\mathrm{KO}$ and wild-type mice. Quantitative RT-PCR of specific mRNAs showed an increased expression of p66-shc in the cortex and whole brain of $\mathrm{KO}$ mice as compared with the wild-type. The level of 5-lipoxygenase mRNA decreased, whereas the expression of $\operatorname{cox} 1$ and $\operatorname{cox} 2$ was unaffected in $\mathrm{KO}$ mice. The data suggest that GSTM5 could be involved in regulation of stress responses.

Keywords: glutathione S-transferases; knockout mice; brain; gene expression

\section{Accelerated brain-to-blood efflux of glutamate: A novel} approach for the management of neurodegenerative diseases

Teichberg V.I. and Gottlieb M

Dept. of Neurobiology, Weizmann Institute of Science, Rehovot 76100

Abnormally high Glutamate (Glu) levels in brain interstitia and cerebrospinal fluids (ISF/CSF) are the hallmark of several neurodegenerative conditions. We examined here the prediction that a decrease of blood Glu levels should facilitate the naturally occuring process of brain-to-blood Glu efflux and assist the brain to remove the excess Glu from the ISF/CSF into blood. The feasibility of accelerating the brain to blood Glu efflux was studied by using two basic paradigms based on the fate of $[3 \mathrm{H}] \mathrm{Glu}$ infused into brain. In the first, we infused $[3 \mathrm{H}] \mathrm{Glu}$ intracerebroventricularly and followed its appearance in blood before, during and after decreasing blood Glu levels by the intravenous administration of pyruvate and oxaloacetate the respective Glu co-substrates of the blood resident enzymes Glutamate-pyruvate transaminase and the Glutamate-oxaloacetate transaminase, In the other, we performed ventriculo-cisternal perfusions of $[3 \mathrm{H}] \mathrm{Glu}$ and followed its disappearance from brain before, during and afte decreasing blood Glu levels with pyruvate and oxaloacetate. In both cases, the results obtained point out to the same conclusion that the intravenous administration of pyruvate and oxaloacetate that scavenges blood Glu accelerates proportionally the brain-to-blood Glu efflux. On that basis, we propose the use of intravenous pyruvate and oxaloacetate for the treatment of all neurodegenerative conditions in which the excess Glu present in the brain interstitial fluid or cerebrospinal fluid is thought to trigger neuronal cell death and its accompanying neuropathological sequelae. Examples of the neuroprotective and life-saving role of this newly proposed blood Glu scavenging process will be presented.

Keywords: neuroprotection, glutamate scavenging,

brain-to-blood efflux

\section{Ischemic preconditioning involves greater reductive} capacity of the brain and lesser oxidative stress in rats Trembovler V. ${ }^{1}$, Glantz L. ${ }_{2}$ Avramovich A. ${ }^{2}$ Gurvitz V. ${ }^{2}$, Kohen R. Eidelman L.A. ${ }^{2}$ and Shohami E.

${ }^{I}$ Dept. of Pharmacology. Hebrew University. Jerusalem and ${ }_{2}^{2}$ Anesthesiology, Rabin medical Center. Petah Tikva

Background: Brain injury (ischemia, trauma) releases reactive oxygen species (ROS) implicated in secondary brain damage Endogenous low molecular weight antioxidants (LMWA) neutralize ROS and are among the defense mechanisms protecting tissues from oxidative stress. Sub-lethal injur provides protection by a mechanism not fully understood collectively referred to as "preconditioning". This study examines whether brain LMWA are altered in ischemic preconditioning.

Methods: Rats were preconditioned (PC) by 2 min occlusion (or sham surgery) of right middle cerebral artery (rMCAO) using intraluminar suture. $24 \mathrm{~h}$ later arterial blood pressure was reduced to $55 \mathrm{mmHg}$ and rats were subjected to $90 \mathrm{~min}$ of rMCAO (or sham surgery) followed by $5 \mathrm{~min}$ or $4 \mathrm{~h}$ of reperfusion. LMWA were extracted from both hemispheres and the tissue reducing power derived from LMWA was evaluated by cyclic voltammetry (Kohen et al. Methods in Enzymology 300: 285-296 [1999]). Infarct volume was assessed at $4 \mathrm{~h}$ using TTC staining.

Results: PC rats displayed $20 \%$ smaller infarct volume as compared to non-PC rats $(\mathrm{p}<0.05)$. In non-PC rats, at 5 min of reperfusion LMWA levels dropped from $0.209 \pm 0.062$ to $0.149 \pm 0.021 \mathrm{nAmp} / \mathrm{mg}$ protein $(\mathrm{p}<0.05)$, indicating their consumption by massive ROS producion during ischemia. In contrast, in PC rats a 2.5 -fold increase was measured (to $0.531 \pm 0.114 \mathrm{p}<0.001) \quad$ suggesting that mobilization of 
antioxidants occurred at preconditioning, and their availability during the ischemic injury is part of the neuroprotection. At $4 \mathrm{~h}$ of reperfusion the LMWA levels increased by 14 and $18 \%$ in both groups. These findings agree with our previous reports showing that higher LMWA levels after injury are associated with better recovery.

Keywords: cerebral ischemia, preconditioning, oxidative stress, antioxidants

\section{Behavioral impact of hyperhomocysteinemia in}

Apolipoprotein E-deficient mice

Troen A., Shukitt-Hale B., Smith D.E., Selhub J. and Rosenberg I.H.

Jean Mayer USDA Human Nutrition Research Center on Aging at Tufts University

High levels of homocysteine in blood are a risk factor for Alzheimer's disease and cognitive decline in the elderly (Seshadri, S. et al. New England Journal of Medicine 346(7):476-83 [2002]). Homocysteine, a sulfur-containing amino acid, generated as a metabolic by-product of biological methylation reactions, is thought to be toxic to endothelial cells and neurons, and is disposed through reactions that require folate and vitamins B12 and B6 as cofactors (Selhub, J. et al., American Journal of Clinical Nutrition 71(Supplement): 614S-20S [1999]). We used Apolipoprotein E-deficient mice to determine whether nutritionally induced hyperhomocysteinemia would accelerate age-related neurological changes characteristic of these mice. 4-week old, male ApoE-null mice were fed control, B-vitamin (folate, B12 \& B6) deficient, B-vitamin deficient and methionine supplemented, and B-vitamin and methionine supplemented diets for eight weeks. Wild type C57/Bl mice fed a control diet were used to establish behavioral and biochemical reference values. At 8 weeks, mice underwent behavioral testing on a series of psychomotor tests, the Morris Water Maze test of spatial memory and learning, and measurement of home-cage exploration and open-field behavior. The two B-vitamin deficient diets induced significant hyperhomocysteinemia in ApoE-null mice, and were associated with markedly longer escape latencies from the Morris Water Maze, but not with psychomotor performance. A trend for hyperactivity in B-vitamin supplemented ApoE-null mice was observed in nocturnal open-field activity and in measures of psychomotor function. Behavioral differences were not observed between ApoE-null and wild-type mice fed control diets.

Keywords: nutrition, homocysteine, behavior, dementia ApoE, mouse

Involvement of the monoaminergic neurotransmitters systems in cooperative coordinated behavior in laboratory rats

Tsoory M. ${ }^{1}$, Youdim MBH. ${ }^{1}$ and Schuster R. ${ }^{2}$

${ }^{1}$ The Eve Topf and USA National Parkinson Foundation Centers of Excellence for Neurodegenerative Disease and Dept. of Pharmacology, The Bruce Rappaport Faculty of Medicine, Technion Institute of Technology; ${ }^{2}$ Dept. of

Psychology. University of Haifa

The motivation to cooperate is explained by two contrasting approaches. An 'INDIVIDUAL' perspective focuses on the individual's behaviors and outcomes. A 'SOCIAL' perspective suggests a distinct "social reward" mechanism influenced by the presence and behaviors of participants.

Cooperation was studied using a laboratory rat model of cooperative coordinated shuttling. Previous research found that cooperative coordinated shuttling is a social act and that it is preferred over individual shuttling.

Shuttling coordination, social behaviors and activation of the dopaminergic, noradrenergic and serotonergic systems in the frontal cortices, and hypothalamus (assessed by HPLC method) were compared across five groups.

COOP: pairs shuttling coordinately; S/I: 'pairs' shuttling uncoordinatedly; IND: individual shuttling; $\mathrm{NC}$ : individual not required to shuttle, and $\mathrm{C}$ : control group.

The results support the "SOCIAL" perspective. In both COOP and S/I groups, intra- pair asymmetries were related to aggressive behaviors, but the COOP group was characterized by more frequent use of non- aggressive social behaviors

The monoamine data supports the 'social reward' hypotheses. Social factors such as pair-partner's presence and social behaviors affect these systems in a pattern distinct from individual behaviors. Hypothalamus NE and 5HIAA data point to different states of arousal attributed to the presence and behaviors of the partner. Differences between 'SOCIAL' and 'NONSOCIAL context groups in right frontal cortex DA concentration, point to its involvement in rewarding social behaviors. Lower levels of $5 \mathrm{HT}$ found in left frontal cortex of the COOP group compared to the $\mathrm{S} / \mathrm{I}$ group, may relate to the more frequent non-aggressive social behaviors that characterizes cooperation.

Keywords: cooperative behaviors, social behavior, coordinated behaviors, social reward, monoamines

The functional relevance in pancreatic $\beta$ islet cells of domains of the delayed rectifier Kv channels, Kv1.1 and Kv2.1, that bind in vitro syntaxin $1 A$ and SNAP-25

Tsuk S. and Lotan I.

Tel Aviv University, Tel-Aviv 69978

Recently, we have shown that the voltage gated $\mathrm{K}^{+}$channels Kv1.1 and Kv2.1 interact directly with the SNARE proteins of the exocytotic machinary. In this study we have mapped the binding regions within the channels that are involved in this interactions. We have shown that syntaxin $1 \mathrm{~A}$ binds the $\mathrm{N}$ terminus of the Kv1.1 channel, preferentialy the T1'A' region of the assembly domain. SNAP-25's pattern of binding to $\mathrm{Kv} 1.1$ is similar. In collaboration with the lab of Dr Gaisano from the University of Toronto (1) we have shown that SNAP-25 inhibits the Kv1.1 activity in insulinoma HIT-T15 cells, and the inhibitory effect is relived by application of GSTfusion proteins corresponding to the $\mathrm{N}$ terminus and to the T1'A' domain, but not by the C terminus or GST itself. These results support the notion that SNAP-25 exerts its inhibitort effect on Kv1.1 through interaction with the $\mathrm{N}$ terminus, specifically the Tl'A' domain.

Next, we characterized the interaction with Kv2.1 and showed that syntaxin $1 \mathrm{~A}$ binds the $\mathrm{C}$ terminus preferentially the $\mathrm{Cl}$ domain (aa 411-632). The binding of syntaxin to the $\mathrm{Cl}$ domain was determened to be saturable with a stochiometry of $1: 3$, implying that 1 molecule of syntaxin binds a tetramer of Kv2.1 subunits.

We than characterized the binding of SNAP-25 to the channel and showed that it binds both the $\mathrm{N}$ and $\mathrm{C}$ termini, preferring the $C 2$ domain (aa 633-853). In collaboration with Dr Gaisano (2) we have shown that SNAP-25 inhibits the Kv2.1 activity in $\beta$-cells and upon application of fragments coresponding to the $\mathrm{N}$ terminus this inhibition was relived

These results support a causative relationship between the functional effects of SNAP-25 on Kv1.1 or Kv2.1 and its binding to the channels in insulinoma or $\beta$ cells, respectivly. Causative relationships regarding syntaxin $1 \mathrm{~A}$ are yet to be determined

(1) Ji et al., J.Biol. Chem;277: 20195-204 (2002)

(2) MacDonald et al., Mol. Endocrinol. In press. Keywords: Kv channels, syntaxin 1A, SNAP-25, SNARE complex

Representation of stimulus probability by primary auditory cortex neurons

Ulanovsky N., Las L. and Nelken I.

Dept. of Physiology - Hadassah Medical School, and the Interdisciplinary Center for Neural Computation, Hebrew University, Jerusalem

The ability to detect rare auditory events may be critical for survival. Furthermore, stimulus statistics strongly influences the optimal neural code. We presented sequences of tones at two frequencies $\mathrm{fl}, \mathrm{f} 2$, and manipulated the frequency interval df between them ( $d f=37 \%, 10 \%$ and $4 \%$ ), and the relative abundance of the two tones $(p=90 / 10 \%, 70 / 30 \%$ and $50 / 50 \%)$ Neural activity was recorded in primary auditory cortex (A1) and auditory thalamus (MGB) of cats under halothane anesthesia.

Neurons in Al, but not in the MGB, responded more strongly to a sound when it appeared rarely in a sequence than to the same sound when common. The difference in responses to common and rare sounds was a result of stimulus-specific decline in the responses to the common sound (stimulus-specific adaptation, SSA). This difference was positively correlated with $\mathrm{df}$, and negatively correlated with the abundance of the rare tone. Thus, Al neurons are sensitive to global stimulus statistics.

Significant differences between responses to common and rare sounds were found in many neurons even for the smallest frequency interval of $4 \%$. These neurons exhibited therefore hyperacuity, a frequency resolution that is an order of magnitude better than receptive field width in either Al or the auditory periphery

We hypothesize that this form of SSA is a neural correlate of mismatch negativity, an important auditory event-related potential, implicated in sensory memory. Our results suggest that auditory cortex neurons, in addition to processing the acoustic features of sounds, may play a role in sensory memory and in novelty detection. 
Keywords: auditory cortex; physiology; adaptation; mismatch negativity

\section{Modeling neuronal degeneration and autoimmune} protection in the central nervous system (CNS)

Uziel A. ${ }^{1,2}$, Kipnis J. ${ }^{2}$, Butovsky O. ${ }^{2}$, Shaked I. ${ }^{2}$, Mizrahi T. ${ }^{2}$ Neumann A. $^{1}$ and Schwartz M. ${ }^{2}$

${ }^{1}$ Bar-Ilan University, Ramat-Gan; ${ }^{2}$ Weizmann Institute of Science, Rehovot 76100

Neuronal degeneration in the CNS is often characterized by a progressive degeneration, which continues even after the primary causative factor has been removed. This is attributed to the self-destructive process, Secondary Neuronal Degeneration (SND). It was discovered (Moalem et al., Nat. Med. 5:49-55 [1999]) that autoimmune T-cells could rescue neurons from SND. Paradoxically, the same cells can also induce the autoimmune disease EAE, resulting in neuronal damage. We develop a set of mathematical models, that might explain the mechanisms of SND, and the possible mechanisms for immune protection and destruction. We show that the 'SIR' model of epidemics with some modifications can qualitatively simulate SND results. Similar to SND, an epidemic is also a self-perpetuating process whereby a disease spreads from an initial population of infected people to healthy people. To study the effects of the immune system, we extended the 'SIR' model by adding two populations with biological effects that seem to play an important role in these processes; one population attacks neuronal tissue by eradicating the injured and damaging the healthy bystanders, and the other heals the injured. With the extended 'SIR' we qualitatively reproduce important effects of the immune neuroprotection, including the dual effect of the autoimmune $T$ cells of both benefiting and harming nerve cells. Moreover, we predict that to benefit the damaged tissue, the amount of injected T cells should not be constant but should depend on the severity of the injury.

Keywords: autoimmunity; neuroprotection; secondary damage; nerve injury

\section{Hyperdense basilar arthert - a fatal prognostic sign} Vander T. ${ }^{1}$, Shelef ${ }^{2}{ }^{2}$, Benifla M. ${ }^{2}$, Herishanu Y.O. ${ }^{1}$ and Herzanu Y.

Dept. of neurology and Dept. of radiology ${ }^{2}$, Soroka Medical Center Faculty of Health Sciences, Beer-Sheva

Basilar artery thrombosis is rightly considered as a medical emergency with untreated patients having mortality rate approaching $100 \%$. Non-contrast computer tomography (NCCT) may show a hyperdense basilar artery (HBA) before brainstem infarct is visualized.

In a review of admission records and NCCT of 20 patients admitted to Soroka Medical Center between 1999-2002 with fatal basilar stroke, HBA was found in 10 patients. Eight patients had HBA before clear infarct was demonstrated. This group of patients was significantly younger than patients without HBA. Latency time between admission and deterioration was significantly shorter in patients with HBA. A higher density of the basilar artery was found and measured by comparison to other level of the basilar artery, to the other main artery at the same patients and to patients of matched group with non fatal brain stem infarct.

HBA is a fatal prognostic sign found in relatively young patients. Early thrombolitic treatment should be considered in this catastrophic entity in order to improve outcome.

\section{Patterns of eye movements in stereo motion induced} blindness

Wagner $\mathrm{M}$.

Charles E. Smith Family Laboratory for Collaborative Research in Psychobiology, at the Hebrew University of Jerusalem, Israel, and the College of Judea and Samaria, 44837 Ariel; mwagner@shum.huji.ac.il

Bonneh et al reported a phenomenon of visual disappearance When a global moving pattern is superimposed on high contrast stationary stimuli, the latter disappear and reappear alternately. They termed the phenomenon motion induced blindness MIB results showed that it is unlikely to reflect retinal suppression or sensory masking, but rather is a result of a conflict generated between cortical representations of dissociated stimuli which shifts the system dynamics into a winner-takes-all mode. Other interpretations refer to attentional mechanisms, which cannot be allocated or divided between dissociated elements at the same spatial location and at the same time. In a previous study we have detected relations between eye-movement patterns and gaze-directions, with subject's reports of display component disappearance during a MIB situation. We repeated these studies with stereo MIB. The stationary MIB targets and a rotating distractor dot pattern (tow rotation rates) were perceived as superimposed surfaces and dissociated by 6 stereo disparities. MIB was reported with distractor either in front or behind MIB targets. There was a dramatic saccade-rate decline during MIB periods. Distractor rotation rate affected MIB periods, saccade rate and binocular divergence. High distractor rotation rate generated better binocular divergence to the more distant distractor displays, and a more pronounced saccade rate difference between MIB and No-MIB periods. Results have also revealed effects of time schedule / eye movement dependence on target appearance and disappearance (e.g. target reappearance preceded by a specific eye movement features) Results support the view that attentional mechanisms underlie the MIB phenomena.

Keyword: psychophysics eye-movements

Gene and protein expression profile homology of anti- and pro-apoptotic activity of dopamine, R-apomorphine, EGCG and melatonin in human neuroblastoma cells Weinreb O., Mandel S., Levites Y. and Youdim MBH. Eve Topf and USA National Parkinson Foundation Centers of Excellence for Neurodegenerative Diseases Research and Dept. of Pharmacology and Rappaport Family Research Institute, Technion-Faculty of Medicine, Haifa 31096

Oxidative stress is considered responsible for the degeneration of dopaminergic neurons in the substantia nigra pars compacta in Parkinson's disease (PD) and its animal models Anti-oxidants have concentration dependent neuroprotective and pro-apoptotic activities in models of PD. The aim of our study was to determine gene- protein pathways of the latter activities with structurally different antioxidants, dopamine (DA), R-apomorphine (R-APO), melatonin and green tea polyphenol (-)-epigallocatechine-3-gallate (EGCG) in neuroblastoma (SH-SY5Y) cells, employing cDNA micro-array gene expression technique and quantitative real-time PCR. We demonstrate a concentration-dependent correlation between R-APO, DA, melatonin and EGCG and modulation of cell survival/cell death-related gene pathways Conversely to the effects of low concentrations $(1-10 \mu \mathrm{M})$, where an anti-apoptotic response was manifested pro-apoptotic pattern of gene expression was observed at high concentrations $(50,500,50$ and $50 \mu \mathrm{M}$, respectively) of the antioxidants (e.g. increase of caspases, fas and gadd45). Protein analysis of Bcl-2, Bax and activated caspase- 3 confirmed the gene changes. Melatonin has an extremely low pro-apoptotic activity compared to DA, R-APO and EGCG, which may be partially explained by the observation that high concentration of melatonin, did not significantly affect the expression of the conserved group of mitochondrial Bcl-2 family members, especially $\mathrm{Bcl}-2$ and Bax. Our results provide for the first time new insights into the molecular events involved in the dose dependent activities of catechols, indole amine and phenolic compounds, reputed to have neuroprotective activity at low and neurotoxicity at high concentrations, in cell cultures and in vivo models of neurodegenerative diseases.

Keywords: antioxidants, apoptosis, neurodegenerative diseases, cDNA array

\section{Alteration in the activity of the \\ hypothalamic-pituitary-adrenal axis and behaviour by prenatal stress \\ Weinstock M.}

Dept. of Pharmacology, Hebrew University, Hadassah Medical School, Jerusalem

Retrospective studies have shown that exposure of pregnant women to adverse life events, like marital conflict, war, major disasters, like an earthquake or flood, increases the likelihood of behavioural disturbance in their infants and of a higher incidence neurotic and depressive symptoms in adulthood Prenatally-stressed (PS) rats and monkeys also have disturbances in their behaviour characterized by heightened anxiety in novel, intimidating environments and fail to cope with life-sustaining activities such as food seeking and maternal care under stress. Like depressed human subjects, PS rats show abnormalities in sleep patterns, circadian rhythm, decreased hedonic behaviour (sweet preference), and develop learned helplessness more rapidly than controls. Gestational stress in women and experimental animals elevates circulating corticotropin-releasing hormone $(\mathrm{CRH}), \mathrm{ACTH}$ and glucocorticoids and alters the normal relationship between $\mathrm{ACTH}$ and beta-endorphin. It also brings about a reduction in hippocampal glucocorticoid receptors in the offspring and in the feedback inhibition of CRH, leading to a further increase in blood levels of glucocorticoids. The depressive symptoms, hyperanxiety and abnormal regulation of the hypothalamic-pituitary-adrenal (HPA) axis can be significantly 
decreased by chronic treatment with antidepressants in humans. In many of such subjects they also correct the abnormal feedback regulation of the HPA axis. In adult PS rats, acute intracerebral administration of a $\mathrm{CRH}$ antagonist can reduce their hyperanxiety. Chronic oral administration of antidepressants to PS rats from puberty is also effective in normalizing the anxiogenic behaviour as well as the HPA axis regulation at adulthood.

Keywords: prenatal stress; hyperanxiety; corticotropin releasing hormone; antidepressant

The POU4F3 transcription factor in human hereditary

Weafness ${ }_{\text {Weiss. S. }}{ }^{1}$, Gottfried I. ${ }^{1}$, Xiang M. ${ }^{2}$, Dawson. S.J. ${ }^{3}$ and Avraham K.B. ${ }^{1}$

${ }^{I}$ Dept. of Human Genetics and Molecular Medicine, Sackler School of Medicine, Tel Aviv University, Tel Aviv; ${ }^{3}$ Center for Advanced Biotechnology and Medicine, and Dept. of Pediatrics, UMDNJ Robert Wood Johnson Medical School, Piscataway, New Jersey, USA; ${ }^{3}$ Dept. of Molecular Pathology, University College London Medical School, London, UK. A mutation in the POU4F3 gene is responsible for DFNA15 (OMIM 602459), autosomal dominant nonsyndromic hearing loss, in an Israeli family. We are now trying to understand the exact mechanism by which the mutation causes this phenotype. Since this protein is only expressed in the hair cells of the inner ear, we were unable to obtain material from affected individuals to study the mechanism. Therefore, we cloned the wild type and mutant POU4F3 into expression vectors and transfected them into HEK293, cos-7, and cochlear cells. Both the wild type and truncated POU4F3 were stably translated. However, the truncated POU4F3 protein was more abundant when compared to the wild type product. Metabolic labeling experiments (pulse-chase) have indicated that the mutant form half life is longer than the wild type form, suggesting that accumulation of this protein will cause cellular damage over time. Luciferase experiments indicated that the mutant protein could not activate the Snap25 promoter to the same extent as the wild type protein. In situ immunofluorescence revealed that most of the truncated POU4F3 protein was localized in the cytoplasm, while the wild type form was in the nucleus. We have recently isolated the putative nuclear localization signals (NLSs) of POU4F3 and shown that both a monopartite and bipartite NLS is required for complete nuclear localization of this transcription factor. Thus our data clearly demonstrates that the majority of the mutant form of POU4F3 is unable to enter the nucleus, presumably preventing it from regulating transcription of its targets in the nucleus.

Keywords: transcription factor, protein stability, NLS, deafness

Role of the 5HT3 receptor in the nucelus accumbens in neurochemical alterations in an animal model of depression Weizmann Y., Geffen-Aricha R., Dremencov E. and Y adid G Faculty of Life Science, Bar Ilan University, Ramat-Gan

Current hypotheses on the etiology of depression attribute the disorder to alterations in serotonin neurotransmission However, the relationship between these alterations and depressive behavior is poorly understood. Conversely, an interaction between the serotonergic and dopaminergic systems in the nucleus accumbens (NAc) has been established. It has been demonstrated that there is a dramatic loss of 5HT-induced dopamine release in the NAc of an established rat model for depression (Flinders Sensitive Line, FSL) that can be reversed with antidepressant treatment. $5 \mathrm{HT}_{3}$ receptors are central mediators of the serotonin-dopamine interaction in the NAc. Therefore, changes in the $5 \mathrm{HT}_{3}$ receptor function can affect depressive behavior.

Our aim was to examine the role of the $5 \mathrm{HT}_{3}$ receptor in alterations of 5HT-induced dopamine release in the NAc of FSL rats. Dopamine release was lower in FSL rats compared to controls following acute $5 \mathrm{HT}_{3}$ agonist administration. Although there was no difference in $5 \mathrm{HT}_{3}$ receptor density in FSL compared to control rats, the receptor affinity was significantly lower $(40 \%)$ in FSL rats. Chronic treatment of FSL rats with antidepressants increased the affinity of the $5 \mathrm{HT}_{3}$ receptor to the level of controls, but did not markedly affect the receptor affinity in control rats. Our findings associate $5 \mathrm{HT}_{3}$ receptor with an animal model of depressive behavior. Therefore, $5 \mathrm{HT}_{3}$ receptor may be a target for future treatment of depression

Keywords: depression, nucleus accumbens, 5HT-3
Quantitative analysis of thalamocortical synapses in adult mouse barrels

White E.L., Weinfeld E., Lev D.L.

Zlowtowski Center for Neuroscience, Dept. of Morphology,

Faculty of Health Sciences, Ben-Gurion University of the

Negev, Beer-Sheva

This study focused on the synapses made by thalamocortical afferents to barrel cortex in the adult mouse. The aim was to determine precisely the numbers of boutons per axon length, the number of synapses per bouton, and the identities of postsynaptic elements. Axons were labeled by the anterograde transport of lysin-fixable biotinylated dextran amine (BDA) injected in vivo into the ventrobasal thalamus. Labeled axons in the posteromedial barrel subfield were examined by light microscopy and then reconstructed in three dimensions to assess the spatial distribution of their synapses. All thalamocortical synapses were of the asymmetrical type Generally, thalamocortical synapses were formed at axonal varicosities, however, some synapses occurred at cylindrical portions of thalamocortical afferents. Preliminary results indicated that axonal varicosities form from 2 to 3 synapses. As in developing barrels, the ratio of axospinous to axodendritic synapses was $4: 1$. Comparison of the synaptic organization of thalamocortical afferents at early postnatal stage (P11, Lev et al., 2001) with the adult, support the notion that thalamocortical synaptic connectivity is specified early on in development.

Supported by Israel Science Foundation grant 52/00-2.

Keywords: somatosensory, axon, thalamus, afferent

Contrast discrimination learning with uncertainty
Wilkonsky A. ${ }^{1,2}$ Adini ${ }^{1}$. Sagi ${ }^{1}$. and Tsodyks $M$.

'Dept. of Neurobiology, Brain Research, Weizmann Institute of Science, Rehovot 76100; ${ }^{2}$ Rappaport Medical School. Technion. Haifa

Performance on perceptual tasks improves with practice However, in vision, contrast discrimination thresholds show a remarkable stability when a large range of contrasts $(0-0.6)$ is practiced. There are two known exceptions: (a) when the practiced target is surrounded by flankers (Adini et al 2002), (b) when practicing with a single base contrast (Cong et a 2002). The improvement can be explained by increasing the gain of contrast transduction and/or by optimization of discrimination strategies applied to the specific contrast level(s) used during practice. To separate between the two accounts we measured contrast discrimination thresholds before and after learning in conditions where the observers could not predict the target contrast (contrast uncertainty). Learning effects based on plastic changes in the basic sensory mechanism, but not on contrast dependent strategies, are expected to survive such an experimental manipulation. The pre-learning tests (using localized gratings, Gabor signals) showed the expected stable performance with typical TvC (threshold vs contrast) functions. Next, observers were trained to discriminate between two slightly different contrasts keeping the lower (base) contrast constant $(0.5)$ Discrimination thresholds were halved during practice However, this improvement was found to be specific to the trained condition and post-training tests with contrast uncertainty showed no improvement. A second group of observers practiced the full contrast range with the target embedded in a chain of flankers, showing the expected improvement in contrast discrimination. This learning effect was found to transfer to the post-learning test with contrast uncertainty. It seems that the human visual system can use both high and low level modifications to improve contrast discrimination. The specific choice made by the system may depend on the number of contrast levels used and their spacing. as well as on previous experience.

Keywords: perceptual learning, vision, contrast discrimination, context, lateral interactions

\section{Induction of experimental autoimmune myasthenia gravis} (EAMG) by a lipopolysaccharide-acetylcholine receptor peptide conjugate

Wirguin I. ${ }^{1}$, Bersudsky M. $^{1}$, Sicsic C. ${ }^{2}$ and Brenner T. ${ }^{2}$ Depts. of Neurology, 'Soroka University Medical Center. Beer-Sheva and ${ }^{2}$ Hadassah University Hospital, Jerusalem

Molecular mimicry is implicated in the pathogenesis of autoimmune disorders. For example, in Guillain-Barr ${ }^{7}$ syndrome the immune attack on nerve antigens is attributed to cross reactivity of carbohydrates present in Campylobacter jejuni lipopolysaccharides (LPS) and in gangliosides. We have shown that covalent bonding of an LPS molecule to gangliosides enhances the immune response to self-gangliosides. 
Objective: To test the hypothesis that LPS bound antigens could overcome tolerance to protein antigens.

Methods: The decapeptide WNPDDYGGVK, (the main immunogenic region-MR) of the $\alpha$ chain of acetylcholine receptor (AChR) was conjugated to Salmonella Re-LPS by heterodimeric cross-linking. Female Lewis rats subcutaneously presensitized by purified Torpedo AChR or MIR on days 1 and 21 , received intraperitoneal injections of $10 \mu \mathrm{g}$ of MIR-LPS conjugate on day 28 . Control rats received MIR or Re-LPS only. Animals were tested by repetitive nerve stimulation, treadmill exercise tolerance and immunoassays for $\mathrm{AChR}$ antibodies.

Results: 15/25 LPS-MIR injected rats showed pathological decrements to repetitive nerve stimulation at $3 \mathrm{hz}$ compared to $2 / 25$ in control rats. 4/12 MIR-LPS injected rats were unable to complete 10 minutes of treadmill exercise compared to $1 / 13$ of controls. Rat AChR antibodies were detected by RIA in 3 of the MIR-LPS injected rats and in none of the controls.

Conclusion: MIR-LPS conjugate injections can induce EAMG without additional adjuvants. LPS was shown to have adjuvant like effects in the induction of autoimmune phenomena but the molecular bond of LPS to antigen appears to play an important role in overcoming tolerance to self antigens such as AChR. Keywords: myasthenia gravis, molecular mimicry, lipopolysaccharide-peptide conjugates

Impaired interleukin-1 (IL-1) signaling is associated with increased morphine sensitivity

Wolf G. ${ }^{1}$, Yirmiya R. ${ }^{1}$ and Shavit $Y{ }^{1}$

Dept. of Psychology, Hebrew University, Mount Scopus, Jerusalem 91905

The cytokine IL-1 modulates pain perception in various inflammatory and infectious conditions. IL-1 is known to induce several hyperalgesic mediators, including nerve growth factor and substance $P$. These mediators are also induced by morphine, and they may counteract the analgesic effect of morphine, and possibly participate in development of morphine tolerance. We have recently demonstrated that mice with knockout of the IL-1 receptor type I (IL-1rKO), or the IL-1 receptor accessory protein, as well as mice with transgenic overexpression of $\mathrm{IL}-1$ receptor antagonist, exhibit reduced baseline pain sensitivity compared with their wild-type (WT) controls and parent strains, suggesting that impairment in IL-1 signaling disturbs the balance between analgesic and hyperalgesic systems.

The present study tested the hypothesis that IL-1 influences morphine-induced analgesia. We assessed the effects of morphine on pain sensitivity in IL-1rKO and WT mice, using the hot-plate test. A dose of $4 \mathrm{mg} / \mathrm{kg}$ morphine was injected subcutaneously, and pain sensitivity was measured $30,90,150$ 210 , and 270 minutes later. Percent analgesia was calculated using the formula: [(after-injection latency - baseline latency)/(cutoff latency - baseline latency) ${ }^{*} 100$. As expected IL-IrKO mice displayed lower baseline pain sensitivity compare with WT mice ( 27.51 vs. $12.24 \mathrm{sec}$, respectively). Morphine-treated IL-1rKO mice displayed greater percent analgesia compared with WT mice treated with morphine $(100 \%, 100 \%, 59.6 \%, 48.6 \%, 5 \%$ vs. $63.9 \%, 28.3 \%, 27.6 \%$ $18.1 \%, 7.5 \%$, at the different time points, respectively). These findings suggest that in addition to its involvement in basal pain sensitivity, IL-1-signaling also counteracts the analgesic effect of acute morphine.

Keywords: interleukin-1, morphine, pain sensitivity, analgesia

Characterization of the role of the $\mathrm{C}$-terminus in the interaction of the voltage-gated $K^{+}$channels $K v 2.1$ and Kv2.2 with the proteins SNAP-25 and syntaxin

Wolf-Goldberg T., Michaelevski I., Chikvashvili D. and Lotan I. Dept. of Physiology and Pharmacology, Sackler Faculty of Medicine, Tel-Aviv University, Tel Aviv 69978

Recently, we have shown that presynaptic Kvl.1 and Kv2.1 interact directly with SNAP-25 and syntaxin in synaptosomes and neuroendocrine cells-PC12, respectively. Integrated electophysiological and biochemical analyses in Xenopus oocytes reveal effects on the Kv2.1 biophysical parameters due to its interactions with those proteins. These studies indicate that the C-terminus might have an important role in the interaction of the channel with the proteins. To further characterize the role of the C-terminus we study the interaction of the synaptic proteins with the Kv2.2 channel which is the most closely related to $\mathrm{Kv} 2$.1. The $\mathrm{NH}_{2}$ - terminal cytoplasmic domains and the hydrophobic cores of both channels, which contain six transmembrane segments, have a $84.2 \%$ amino acid identity but the remaining $\mathrm{COOH}$-terminal cytoplasmic portion starting at threonine 535 in Kv2.1 displays only $21 \%$ of identity. Our results point out to a substantial similarity between the effects of syntaxin and SNAP-25 on the two channels, however, with some differences. Kv2.1 and Kv2.2 are differentially localized in the brain and in various peripheral tissues, e.g. the greatest density of $\mathrm{Kv} 2.2 \mathrm{mRNA}$ is detected in the olfactory bulb, followed by the cerebral cortex, hippocampus and cerebellum whereas Kv2.1 mRNA levels are highest in the cerebral cortex, followed by the hippocampus, cerebellum and olfactory bulb (Hwang et al, (1992) Neuron Vol 8:473-481). These differences mav help us understand if the two channels may subserve similar functional roles in different organs or they may mediate distinct functions.

Keywords: Kv2.1, Kv2.2, C-terminus, synaptic proteins

Neuropharmacogenetic changes in the depressive brain: Studies in an animal model of depression Yadid G.

Life Sciences Faculty, Bar-Ilan University, Ramat-Gan

Understanding the abnormalities manifested in neurochemical pathways during depressive disorders and the dynamic effects of these abnormalities on the onset of action and efficacy of pharmacological treatments is crucial for the development of effective antidepresssant drugs and therapeutic strategies

Over the last 40 years, eight classes of antidepressants have become available. Nonetheless, none of them improve the efficacy, speed the response or demonstrate long-term effects In addition, studies have suggested many neurotransmitters and neuromodulators as candidate systems involved in depression. Neurotransmission changes in the brain should be monitored dynamically and the cross-talk between these neuronal systems should be at multiparametrical levels: neurophysiological. neurochemical and neurogenetic.

The present review will discusses recent findings concerning alterations in the neurobiology of the brain that accompany depressive behavior and effective antidepressant treatment in a unique animal model of depression (Flinders Sensitive Line of rats).

Keywords: depressive behavior, neurogenetics,

neurochemistry, neurophysiology

\section{Working memory for multiple stimuli in monkeys} Yakovlev V. ${ }^{1}$, Orlov T. ${ }^{1}$, Amit D. ${ }^{2}$, Hochstein $\mathrm{S}^{1}{ }^{1}$ and Zohary E. ${ }^{1}$

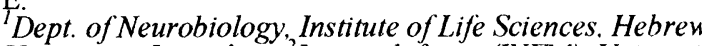
University. Jerusalem: 'Isituto di fisica (INFM). Universita' di Roma. La Sapienza, and Racah Institute of Physics. Hebrew University, Jerusalem

We studied how monkeys hold several familiar stimuli in working memory. Two Macaque monkeys were trained on a modified delayed-match-to-sample task with multiple samples. Monkeys viewed a sequence of stimuli on a computer monitor and released a lever when any one of the sample stimuli in the sequence was presented for a second time within the trial - i.e. a "match" stimulus. Trials had 2-8 stimuli with 1,2 or $3 \mathrm{~s}$ inter-stimulus intervals. Two type of errors occurred:

1. "Misses" when the monkev failed to respond to a "match" presentation. Distribution of these errors depended on the position of the "cue" (first presentation of the stimulus which was later matched). The greater the cue-match separation. the poorer the performance. This finding indicates that working memory of a given item erodes with time and/or the number of intervening stimuli. However, for a given cue-match separation, performance improved with sequence length. i.e with number of irrelevant stimuli preceding the cue. This may reflect the rising expectation of reward

2. "False positives" when the monkey erronously responded to an image which was not a repetition of a previously presented stimulus within the trial (a "non-match" stimulus) Distribution of these errors depended on the time and number of stimuli since the beginning of the trial.

Taken together, these two findings suggest that noise associated with the neural dynamics, spontaneously erases items from working memory and induces ghost memories of familiar, unseen stimuli. A detailed model has corroborated this picture.

Keywords: working memory 
Erythropoietin (EPO) is neuroprotective in mice and rats and markedly reduces neuronal apoptosis in an experimental model of closed head injury .

Yatsiv I. ${ }^{, 2}$, Grigoriadis N. ${ }^{3}$, Alexandrovitch AG. ${ }^{1}$, Simeonidou $\mathrm{C}^{4}$, and Shohami E.

Dept. of Pharmacology and ${ }^{2}$ Pediatric Intensive Care, Hebrew University Hadassah Medical Center, Jerusalem, Israel: ${ }^{3}$ Dept. of Neurology and ${ }^{4}$ Pathology, AHEPA University Hospital. Thessaloniki. Greece

Pro-inflammatory brain cytokines, released hours after closed head injury (CHI) have deleterious effect. Anti-inflammatory and anti-apoptotic modalities ameliorate $\mathrm{CHI}$ outcome. Erythropoietin (EPO), a kidney-derived cytokine regulates hematopoeisis, acts as a growth factor and apoptotic inhibitor. Cultured brain cells produce. EPO in response to oxidative stress, and EPO receptor is present on neuronal and brain capillary endothelial cells. This study examines the protective effects of EPO in rats and mice undergoing controlled CHI. Methods: $\mathrm{CHI}$ was induced using a weight-drop device. Clinical status was evaluated by Neurological Severity Score (NSS), which tests 10 or 17 tasks for mice and rats respectively. Animals were treated with 2 doses of i.p. 5,000 units $/ \mathrm{kg} /$ dose $(1 \mathrm{ml})$ of HuEPO, or vehicle $1 \mathrm{~h}$ and $24 \mathrm{~h}$ after CHI. NSS was evaluated at 1,3 and $7 \mathrm{~d}$ post $\mathrm{CHI}$, and compared using a two-tailed student t-test. Brains were analyzed for apoptosis at $7 \mathrm{~d}$. CHI was of similar severity in both rat groups. Recovery was facilitated in the treated mice starting at $24 \mathrm{~h}$ and reaching statistical significance at $7 \mathrm{~d}$ post CHI (NSS $=5.0$ vs 6.625 in treated and control mice, respectively, $p=0.037$ ). Similar recovery pattern was found in $\mathrm{CHI}$ mice. While a considerable neuronal apoptosis and minimal apoptosis of the leukocytes of the inflammatory infiltrate cells was found at the injury site in control animals, a markedly reduced neuronal apoptosis with increased inflammatory cell apoptosis were found after EPO treatment. We propose that EPO-treated animals recovered faster than controls with less neuronal and more inflammatory cell apoptosis compared to controls, probably through a cell-specific anti-apoptotic mechanism.

Keywords: traumatic brain injury, erythropoietin, apoptosis, neuroprotection

Tomosyn is involved in the last stages of the synaptic vesicle cycle

Yizhar O. ${ }^{1}$, Hagalili Y. ${ }^{1}$, Melamed R. ${ }^{1}$, Matti U. ${ }^{2}$, Rettig J. ${ }^{2}$ and Ashery U

${ }^{I}$ Dep. Of Neurobiochemistry, Wise Faculty of Life Sciences, Tel Aviv University, Tel-Aviv 69978: 2Physiologisches Institut, Universitat des Saarlandes, Homburg/Saar, Germany

The process of neurotransmitter release is coordinated by a large number of synaptic proteins and depends on proper protein-protein interactions. $m$-Tomosyn is a brain-specific, $130 \mathrm{kD}$ protein that was identified as a novel binding partner for Syntaxinl. Tomosyn possesses a Synaptobrevin-homology domain and is capable of dissociating Munc18 from Syntaxin1. To investigate the role of Tomosyn in catecholamine secretion, we overexpressed Tomosyn in adrenal chromaffin cells. Using photolysis of caged-calcium, we studied the effect of Tomosyn on the different kinetic components of exocytosis with membrane capacitance measurements. While overall secretion under overexpression of Tomosyn was not different than control, we observed a pronounced difference in the kinetics of secretion. The exocytotic burst was significantly reduced in Tomosyn-overexpressing cells, indicating a decrease in the number of release-competent vesicles. Furthermore, a detailed examination of secretion rates revealed that under Tomosyn overexpression, higher $\left[\mathrm{Ca}^{2+}\right]_{i}$ is required to achieve normal secretion rates. To elucidate the function of the Synaptobrevin-homology domain, we overexpressed a mutated form of Tomosyn, which lacks this domain (Tomosyn $\Delta C C$ ). Under steady-state conditions, deletion of the coiled coil domain slightly reduced the inhibitory effect observed with wild-type Tomosyn. However, following elevation of $\left[\mathrm{Ca}^{2+}\right]_{i}$, the inhibitory effect was abolished altogether. These results suggest that Tomosyn acts at the priming step of the synaptic vesicle cycle. Tomosyn activity might reduce the number of fusion-competent vesicles through its interaction with Syntaxin, by interfering with the formation of fusion-competent SNARE complexes.

Keywords: tomosyn, syntaxin, chromaffin cell, exocytosis
Implications of APP processing, PKC and MARCKS-phosphoration by rasagiline Yogev-Falach M. ${ }^{1}$, Amit T. ${ }^{1}$, Bar-Am O. ${ }^{1}$, Sagi Y. ${ }^{1}$, Weinstock $\mathrm{M}^{2}$ and Youdim $\mathrm{MBH}$.

${ }^{I}$ Eve Topf and NPF Centers of Excellence for Neurodegenerative Diseases Research and Dept. of Pharmacology, Technion-Faculty of Medicine, Haifa; ${ }^{2}$ Dept. of Pharmacology, Hebrew University, Jerusalem

We have recently reported that the anti-Parkinson drugs rasagiline [N-propargyl-1(R)-aminoindan] regulates the secretion of the neuroprotective-neurotrophic non-amyloidogenic soluble form of the amyloid precursor protein (sAPP $\alpha$ ) and initiates cell proliferation in PC-12 and neuroblastoma SH-SY5Y cells. In the present study we provide evidence that protein kinase $C$ (PKC) is critically involved in its APP processing activity, both in vitro and in vivo. Inhibition of PKC by GF109203X $(2.5 \mu \mathrm{M})$ attenuated rasagiline induced SAPP $\alpha$ release in PC12 and SH-SY5Y cells. Indeed, rasagiline $(1-10 \mu \mathrm{M})$ promoted PKC posphorylation, as well PKC translocation in PC12 cells at $1 \mathrm{~h}$ incubation. Administration of rasagiline $(0.1 \mathrm{mg} / \mathrm{kg})$ to mice for 14 days significantly decreased $(\sim 65 \%)$ membrane bound holoprotein APP level in the hippocampus. Concomitantly rasagiline markedly increased PKC $\alpha$ and $\varepsilon$ in the membrane $(\sim 5 \%)$ and the cytosolic $(\sim 60 \%)$ fractions of mice hippocampus. Additionally, rasagiline treatment significantly elevated the levels of myristoylated alanine-rich $C$ kinase substrate (MARCKS), a major substrate for PKC, as well as the levels of receptors for activated C kinase 1 (RACK1). Similar effects on the holo APP and PKC levels were also demonstrated for the two-cholinesterase inhibitor derivatives of rasagiline, TV 3326 and TV3279. These results indicate that rasagiline; TV3326 and TV3279 regulate APP processing by a PKC dependent mechanism. The activation and induction of $\mathrm{PKC}$ and MARCKS by these drugs may have a crucial role not only in their neuroprotective activity, but also a crucial role in their ability to affect neuronal plasticity and spatial learning processes.

Keywords: rasagiline, amyloid precursor protein, protein

kinase C, MARCKS, learning process

Regulation of SAPP $\alpha$ release via MAPK

phosphorylation-dependent mechanism by rasagiline Yogev-Falach M., Amit T., Bar-Am O. and Youdim M.B.H Eve Topf and NPF Centers of Excellence for

Neurodegenerative Diseases Research and Dept. of

Pharmacology, Technion-Faculty of Medicine, Haifa and Dept. of Pharmacology, Hebrew University. Jerusalem

Rasagiline (N-propargyl-1(R)-aminoindan), a neuroprotective monoamine oxidase $\mathrm{B}$ inhibitor in now under phase III clinical trials for Parkinson's disease (PD), and it rescues dopamine neurons from cell death in animal and cellular models of PD. We now show that rasagiline $(1$ and $10 \mu \mathrm{M})$ significantly protected rat $\mathrm{PC} 12$ cells against the $\beta$-amyloid $\left(A \beta_{1-42}\right)$ toxicity. In addition, rasagiline significantly increased $(-3$ fold $)$ the secretion of the non-amyloidogenic soluble form of the amyloid precursor protein (SAPP $\alpha$ ) from PC12 and SH-SY5Y neuroblastoma cells in a dose- dependent fashion. This was blocked by the hydroxamic acid-based metalloprotease inhibitor, Ro31-9790, suggesting mediation via $\alpha$-secretase activity. Specific inhibitor of the mitogen - activated protein kinase (MAPK) pathway, PD98059,.prevented rasagiline induced SAPP $\alpha$ release, indicating the involvement of MAPK-dependent pathway. Furthermore rasagiline dose dependently $(0.1-10 \mu \mathrm{M})$ increased the phosphorylation of $\mathrm{p} 44$ and $\mathrm{p} 42 \mathrm{MAPK}$, which was abolished by PD98059 and by the protein kinase C inhibitor, GF109203X. Structure activity studies with rasagiline derivatives have indicated that the propargylamine moiety, present in rasagiline initiates SAPPo release, as well as MAPK phosphorylation, indicating that the propargylamine moiety is crucial for these effects. These data suggest another novel pharmacological activity for rasagiline. Rasagiline might prove useful in processing APP in favor of non-amyloidogenic pathway, thus reducing the risk of amyloidogenic derivatives formation and therefore it may have potential value in Alzheimer's disease.

Keywords: rasagiline, neuroprotection, amyloid precursor protein, $\alpha$-secretase, MAPK 
Neuroprotective action of rasagiline is dependent on activation of $\mathrm{Bcl}-2, \mathrm{PKC}$ and proteasome complex and inhibition of mitochondrial permeability transition (PT) Youdim M.B.H. ${ }^{1}$, Maruyama W. ${ }^{1}$ and Naoi M. ${ }^{T}$ Eve Topf and N PF Centers of Excellence for Neurodegenerative Diseases Research, Technion, Haifa; ${ }^{2}$ Gifi International Institute of Biotechnology, Gifu, Japan

Our cell culture (PC-12, SH-SY5Y) studies have established that the antiParkinson drug, rasagiline and its optical isomer TVP1022 have relatively potent neuroprotective-antiapoptotic activities in response to several dopaminergic neurotoxins (N-methyl-R-salsolinol and SIN-1). These actions are not dependent on monoamine oxidase (MAO) inhibition, since TVP1022 and other derivatives of rasagiline, which do not inhibit MAO, have similar actions. Some of the events in their neuroprotective action include suppression of the decline in mitochondrial membrane potential, activation of caspase 3 and nuclear translocation of GAPDH. We have now confirmed their ability to stabilize mitochondrial membrane potential in isolated mitochondrial and prevent mitochondrial swelling. This would suggest that rasagiline and its derivatives directly affect the mitochondria, since they inhibit mitochondrial permeability transition (PT), a phenomenon similarly observed in SH-SY5Y cells with over expressed Bcl-2. Rasagiline $(0.1-10 \mu \mathrm{M})$ increases antiapoptotic Bcl-2 gene expression in SH-SY5Y cells. The mRNA and protein levels of Bcl-2 and $\mathrm{Bcl}-\mathrm{xL}$ are also increased significantly, with out affecting those of Bax and Bad. Furthermore rasagiline, similar to $\mathrm{Bcl}-2$ over expression, activates $\mathrm{PKC} \alpha$ and $\varepsilon$ and prevents neurotoxin-induced inhibition of proteasome complex, which in turn results in inhibition of cytochrome $c$ release. In addition, rasagiline increases the gene expression and protein level of glia cell derived neurotrophic factor (GDNF). Our studies have shown that the mechanism of neuroprotective-antipoptotic action of rasagiline depends on inhibition of mitochondrial PT, increased expression of antiapoptotic-cyto-protective (Bcl-2, Bcl-xL and PKC) and neurotrophic genes

Keywords; rasagiline, neuroprotection, mitochondrial

permeability transition, $\mathrm{Bcl}-2$ and $\mathrm{PKC}$ gene expression

\section{Intrinsic bursting in hippocampal pyramidal neurons} lacking apical dendrites

Yue C., and Yaari Y

Dept. of Physiology, Institute of Medical Sciences, Hebrew University-Hadassah School of Medicine, Jerusalem 91120

The intrinsic discharge modes of hippocampal CA1 pyramidal cells vary along a gradient of 'burstiness' from regular firing to spontaneous bursting. The propensity to generate a somatic burst is positively related to the size of the spike afterdepolarization (ADP), generated by a slow inward current that activates during the spike and deactivates slowly thereafter. However, it is not known whether the ADP is locally generated by the somatic spike, is generated remotely at the apical dendrite by the backpropagating spike, or both. To differentiate between these alternatives, we have severed the apical dendrites of CAl pyramidal cells near the soma and examined the firing modes of the amputated neurons in different experimental conditions. Their spectrum of firing modes was similar to that of intact neurons (mostly regular firing cells with a small proportion of bursters). Blocking the muscarinic-sensitive $\mathrm{K}+$ current (IM) with linopirdine markedly enhanced the propensity of amputated neurons to generate bursts, whereas blocking the $\mathrm{Ca} 2+$-activated $\mathrm{K}+$ currents with intracellular (BAPTA) did not. The propensity to generate bursts was also augmented by raising extracellular $[\mathrm{K}+]$ or by removing extracellular $\mathrm{Ca} 2+$. In all conditions, bursting was readily suppressed by phenytoin or by $\mathrm{PKC}$ activation, but was not sensitive to $\mathrm{Ni} 2+(1 \mathrm{mM})$, implicating the persistent $\mathrm{Na}+$ current (INaP) in its generation. Thus, somatic spike backpropagation into apical dendrite is not required for somatic bursting in hippocampal pyramidal cells. Rather, large spike ADPs that trigger somatic bursts are generated at or near the soma once $\mathbb{I N a P}$ is sufficiently larger than IM.

Supported by ISF, BMBF and DFG

Keywords: bursting, persistent $\mathrm{Na}+$ current, $\mathrm{M}$-current, dendrites, hippocampal pyramidal cells
Unilateral pentobarbital microinjection into the rat MPTA induces signs of general anesthsia that are bilaterally symmetrical Zalkind V.I. and Devor M. Dept. Cell \& Animal Biology, Institute of Life Sciences, Hebrew University, Jerusalem

We recently discovered a small area within the rat mesopontine tegmentum (MPTA) at which bilaterally symmetrical microinjection of minute quantities of pentobarbital induces a transient, reversible anesthesia with non-responsiveness to noxious stimuli, flaccid atonia, and absence of the righting reflex. The behavioral suppression is accompanied by slow-wave EEG and presumably, loss of consciousness (Devor and Zalkind, Pain 94:101-12 [2001] ). Here we ask whether microinjection into the MPTA on one side is capable of inducing behavioral signs of general anesthesia on both sides of the body. Using 22 rats that showed anesthesia on bilateral microinjection of pentobarbital, we microinjected this drug, in doses of $100 \mu \mathrm{g}$ in $0.5 \mu \mathrm{l}$ or $200 \mu \mathrm{g}$ in $1 \mu \mathrm{l}$ into MPTA on one side (46 and 44 experiments, respectively). Behavioral results were quantified on motor (posture and righting reflex), and sensory scales (responses to pinching the left and right foot, and the tail). Microinjection sites were located histologically. There were two principal observations. First, anesthesia was induced by unilateral microinjection ( 44 trials) The behavioral suppression was bilaterally symmetrical, and indistinguishable from anesthesia induced by bilateral MPTA or by systemic pentobarbital administration. Second, anesthesia duration, recovery time and mean score following bilateral 100 $\mu \mathrm{g}$ pentobarbital were statistically indistinguishable from those resulting from microinjection of $200 \mu \mathrm{g}$ unilaterally, butgreater than $100 \mu \mathrm{g}$ unilaterally

Conclusion: MPTA on either side of the brainstem controls both ascending and descending pathways, and both motor functions and pain sensation, bilaterally.

Keywords: intracerebral, general anesthesia, unconsciousness, barbiturates, atonia, analgesia, unilateral microinjections

Biophysical and bioinformatic analysis of the cytoplasmic portion of the neuronal cell adhesion protein, gliotactin Zeev-Ben-Mordehai T.1,2, Rydberg E.H. ${ }^{1,2}$, Solomon A. ${ }^{1}$, Toker L. ${ }^{2}$, Botti S. ${ }^{12}$, Silman I. and Sussman J.L. Depts. of ${ }^{\prime}$ Structural Biology and ${ }^{2}$ Neurobiology, Weizmann Institute of Science, Rehovot 76100

In the past decade, a new class of single-span transmembrane neuronal cell adhesion molecules (N-CAMs) has been discovered and characterized. The sequences of the extracellular domains of these molecules show a high sequence similarity to acetylcholinesterase (AChE), and they were thus designated ChE-like adhesion molecules (CLAMs). Their intracellular domains do not show sequence similarity to any known protein, but in the case of one CLAM, neurotactin, this domain was shown to be important for adhesive function. No physicochemical or structural information on any of these molecules has so far been available. We expressed the cytoplasmic sequence of one CLAM, gliotactin (Auld et al Cell 81:757-67 (1995]), which is at its C-terminus (Gli-cyt), in milligram amounts in $E$. coli, purified it, and subjected it to biophysical analysis. Hydrodynamic measurements showed that Gli-cyt has a large Stokes radius $(33$.) relative to its molecular weight $(23.5 \mathrm{kDa})$, indicative of low compactness. $\mathrm{CD}$ measurements, in the near UV, were devoid of ellipticity, indicating absence of tertiary structure, and measurements in the far-UV indicated that it has a "random coil" structure NMR experiments conclusively confirmed that Gli-cyt has a wholly disordered conformation. The amino acid composition of Gli-cyt revealed that it contains both a low percentage of hydrophobic amino acids and a high overall net (positive) charge, as was shown to be the case for other proteins belonging to the class of 'natively unfolded' proteins (Uversky et al, Proteins 41:415-427 [2000]).

Keywords: cholinesterase-like, natively unfolded, intrinsically disorder

Olfactory learning is accompanied by reduction in post burst AHP in CA1 hippocampal neurons

Zelcer I. ${ }^{1}$ and Barkai E.

${ }^{T}$ Zlotowski Center for Neuroscience, Ben-Gurion University, Beersheva: ${ }^{2}$ Center for Brain and Behavior, Haifa University

Rule learning of an olfactory discrimination task in rats is accompanied by reduction in the post-burst after-hyperpolarization (AHP) in piriform cortex pyramidal neurons (Saar et al., EJN 10:1518-1523, 1998). Here we examine whether such reduction also occurs in hippocampal neurons. Water deprived rats were trained in a 4-arm maze to discriminate positive cues in pairs of odors for a water reward. 
AHP amplitude in CA1 hippocampal neurons was recorded in brain slices at different time intervals after the beginning of training. Neurons were depolarized to holding potential of -60 $\mathrm{mV}$, and AHP amplitude was measured following a $100 \mathrm{~ms}$ depolarizing current step that generated 6 action potentials. Olfactory learning-induced reduction in AHP was observed in CA1 neurons as early as 3 days after the rats began their training (i.e. during the time in which they are learning the rule). The averaged AHP amplitude (in $\mathrm{mV}$ ) was $2.88+1.14$, $\mathrm{n}=23$, in neurons from trained rats, $3.54+1.38, n=18$ in neurons from naive and $3.95 \pm 1.51, \mathrm{n}=36$ in neurons from pseudo trained rats $(p<0.05$, one way ANOVA). Similar results were obtained one day after rule learning. Notably three days after rule learning the averaged AHP value in CA1 neurons form trained rats $(4.52 \pm 1.64 \mathrm{mV}, \mathrm{n}=6)$ did not differ from that observed in controls, suggesting that the time course of post-burst AHP reduction and its subsequent return to control values differs between piriform cortex and hippocampal neurons.

We suggest that olfactory learning-related post-burst AHP reduction in CAl neurons may represent a mechanism that enables olfactory rule learning

Keywords: olfactory-learning, hippocampus, CA1, AHP

Solving the EEG inverse problem using genetic algorithms Zilberstein A., Kleks A., Adi-Japha E. and Teicher M.

Bar-Ilan University, Ramat-Gan

Background: The EEG Inverse problem takes an important role in cognitive neuroscience research as well as in clinical applications (e.g. Locating epileptic activities). Several methods have been studied in an attempt to solve this problem (e.g. MNE, BESA, LORETA Pascual-Marqui, Int'l $J$. Bioelectromagnetism, 1(1):75-86 [1999]). None of the methods currently present a satisfying solution.

Objective: Solving the EEG Inverse problem using a new approach based on genetic algorithms.

Methods: Genetic algorithms (Davis. Handbook of Genetic Algorithms, Van Nostrand Reinhold [1991]) are based on the concept of evolution found in nature. A set of population groups of solutions evolve over time to create better solutions according to a set of given genetic rules such as creating mutations, selecting good solutions and combining several good solutions to create better solutions. In our work we used a source model based on dipoles. Every member of a population group is a triple of coordinates that represent the location of the dipole source and its current potential. Mutations change either the location of the dipole or its potential; the selection process is based on a given error threshold between the forward computation of the population groups and the actual EEG measurements. The forward computation is based on the laws of electromagnetism and Maxwell's equations. The initial population set is generated randomly. Previous work using genetic algorithms did not exceed a 2D brain model (Aguiar et al, Proceedings of the 2000 ACM symposium on Applied computing, p.80-84 [2000]) and thus could not be used in real applications.

Results: We tested our algorithm using simulated EEG data The dipoles where simulated by averaging 20 cognitive evoked potential trials and the simulated EEG data was generated by using the forward equations on a homogeneous spherical head model. We tested data sets with 1-3 simultaneous independent dipole sources. The results showed a relatively small error of up to $10 \mathrm{~mm}$, which is similar to the results, obtained by the conventional methods mentioned above.

Keywords: EEG, inverse problem, genetic algorithms 

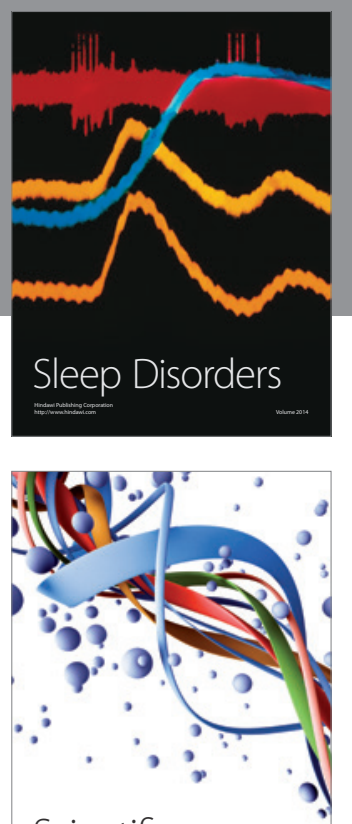

Scientifica
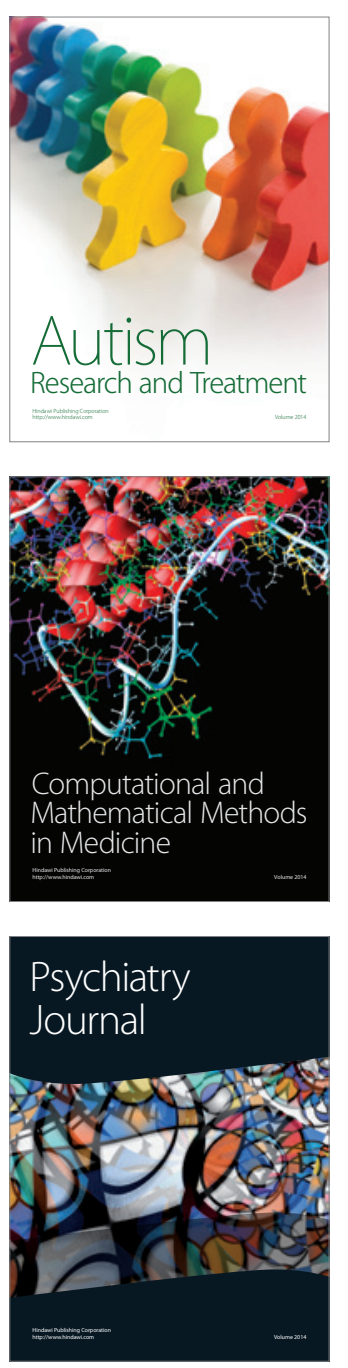
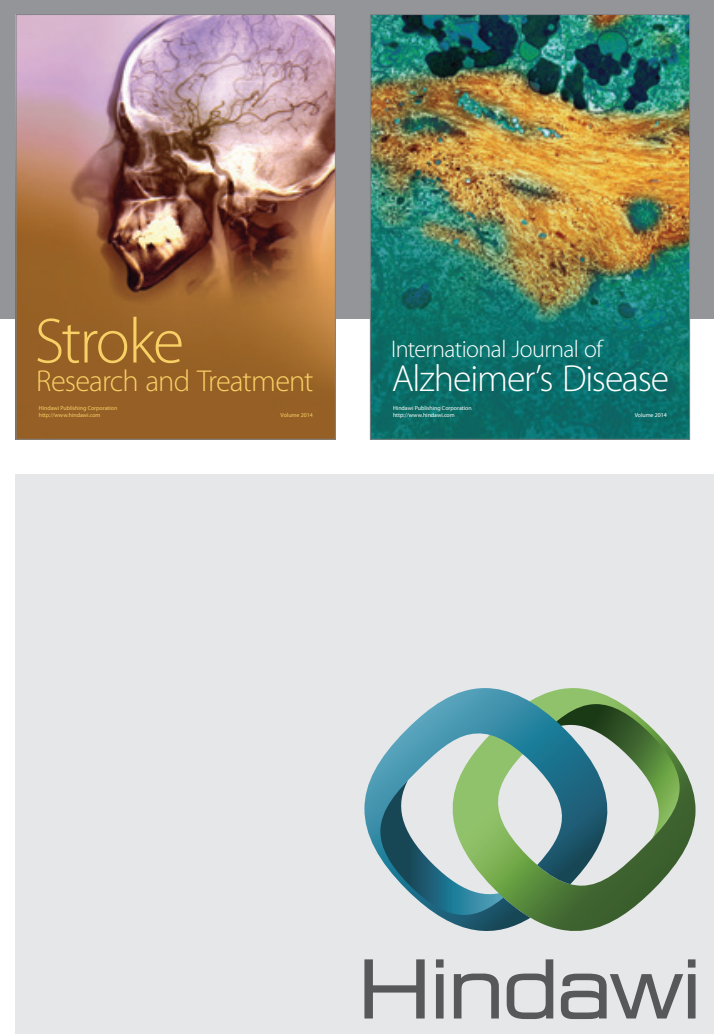

Submit your manuscripts at

http://www.hindawi.com
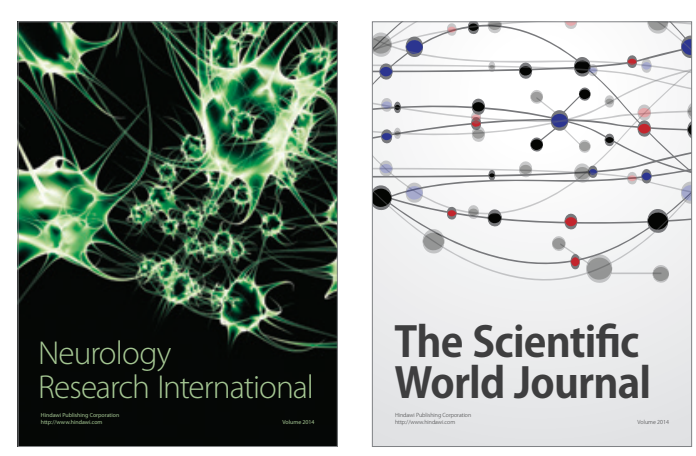

The Scientific World Journal

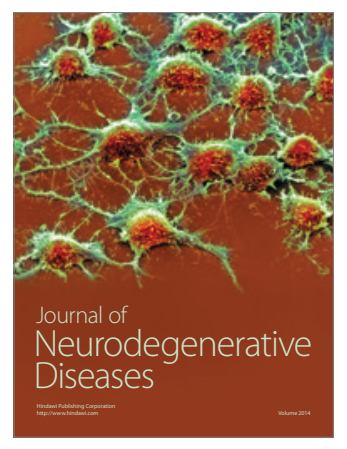

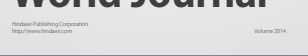

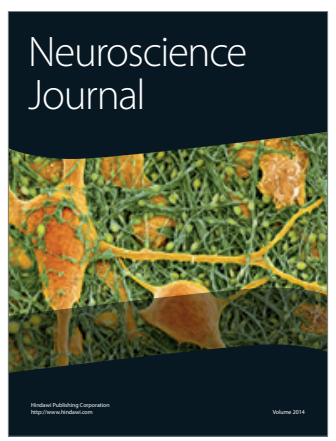

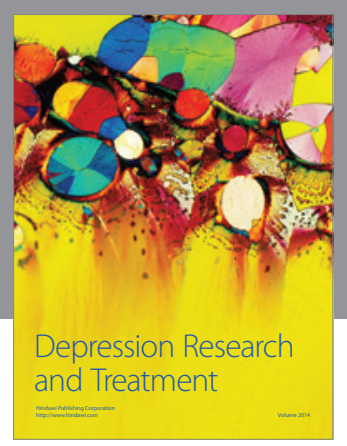
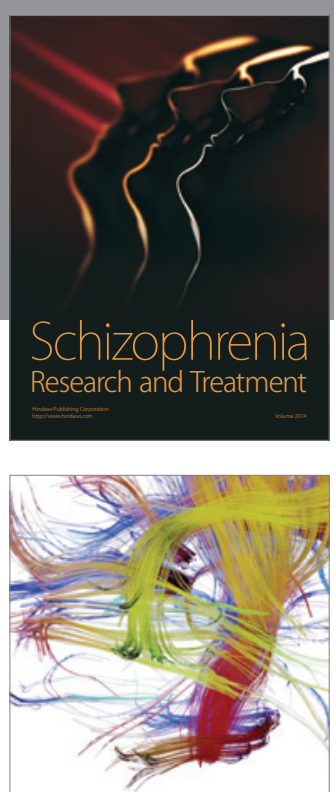

Brain Science

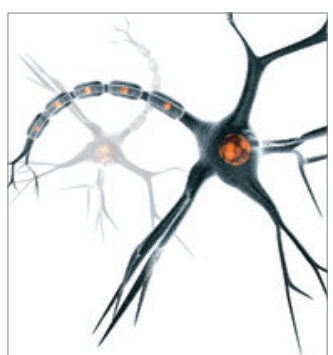

Neural Plasticity
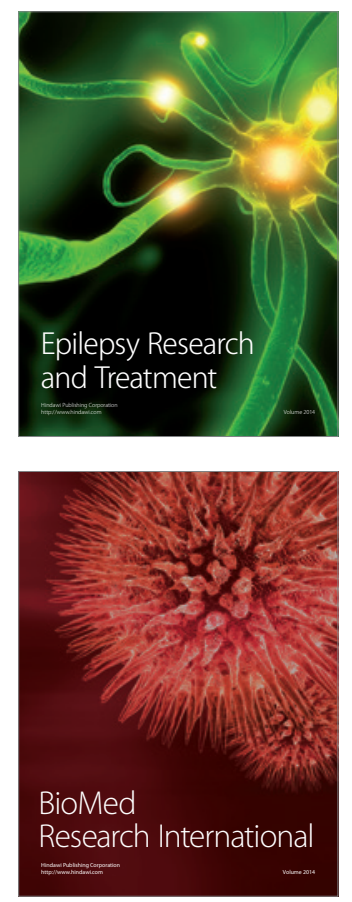

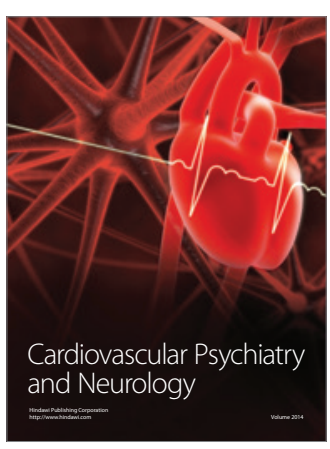

Parkinson's

Disease
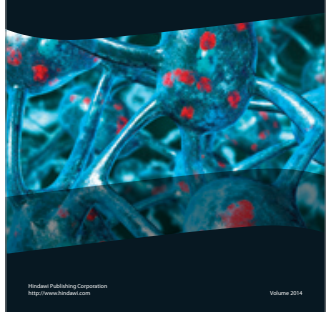